Religioni

e sviluppo

sostenibile a cura di

Ilaria Zuanami

e

Luca Battaglini 


\section{Religioni e sviluppo sostenibile}

\section{Luca Battaglini e Ilaria Zuanazzi (dir.)}

DOI: 10.4000/books.aaccademia.9695

Editore: Accademia University Press

Luogo di pubblicazione: Torino

Anno di pubblicazione: 2021

Data di messa in linea: 28 septembre 2021

Collana: Collana di studi del Centro Interdipartimentale di Scienze Religiose - Università di Torino EAN digitale: 9791280136459

\section{Sbooks}

http://books.openedition.org

\section{Edizione cartacea}

Data di pubblicazione: 1 juillet 2021

EAN (edizione cartacea): 9791280136435

Numero di pagine: 288

\section{Notizia bibliografica digitale}

BATTAGLINI, Luca (dir.) ; ZUANAZZI, Ilaria (dir.). Religioni e sviluppo sostenibile. Nouva edizione [online]. Torino: Accademia University Press, 2021 (creato il 30 septembre 2021). Disponibile su Internet: <http://books.openedition.org/aaccademia/9695>. ISBN: 9791280136459 . DOI: https://doi.org/ 10.4000/books.aaccademia.9695.

(c) Accademia University Press, 2021

Creative Commons - Attribuzione - Non commerciale - Non opere derivate 4.0 Internazionale - CC BYNC-ND 4.0 


\section{Collana di studi del}

CENTRO INTERDIPARTIMENTALE DI SCIENZE RELIGIOSE

Università di Torino

collana diretta da

Ilaria Zuanazzi

comitato scientifico

Paolo Cozzo, Valerio Gigliotti, Alessandro Gusman, Clara Leri, Graziano Lingua, Alessandro Mengozzi, Adele Monaci, Jenny Ponzo, Roberto Francesco Scalon 


\section{Volumi pubblicati}

1. L'Archivio «Erik Peterson» all'Università di Torino.

Saggi critici e inventario

a cura di Adele Monaci Castagno

Alessandria, Edizioni dell'Orso, 2010

2. Sacre impronte e oggetti «non fatti da mano d'uomo» nelle religioni a cura di Adele Monaci Castagno

Alessandria, Edizioni dell'Orso, 2011

3. Dal Mandylion di Edessa alla Sindone di Torino.

Metamorfosi di una leggenda

Andrea Nicolotti

Alessandria, Edizioni dell'Orso, 2011; edizione rivista 2015

4. Il campo religioso. Con due esercizi

Pierre Bourdieu

a cura di Roberto Alciati e Emiliano R. Urciuoli

Torino, Accademia University Press, 2012

5. Itinerari mediterranei fra Iv e Ix secolo

Città-capitale e Deserto-monastico

Atti del Convegno a cura di Beatrice Astrua

Torino, Accademia University Press, 2013

6. Uomini e orsi: morfologia del selvaggio

a cura di Enrico Comba e Daniele Ormezzano

Torino, Accademia University Press, 2015

7. Marcione e il Vangelo (di Luca). Un confronto con Matthias Klinghardt Pier Angelo Gramaglia

Torino, Accademia University Press, 2017

8. Le porte dell'anno: cerimonie stagionali e mascherate animali Enrico Comba e Margherita Amateis

Torino, Accademia University Press, 2019 


\section{Religioni a cura di \\ e sviluppo Ilaria Zuanazzi sostenibile e \\ Luca Battaglini}

aA 
Religioni e sviluppo sostenibile Ilaria Zuanazzi Luca Battaglini

Atti dei Seminari organizzati dal

Centro Interdipartimentale di Ricerca in Scienze Religiose "Erik Peterson" dell'Università di Torino

in collaborazione con la

Facoltà Teologica dell'Italia Settentrionale - Sezione parallela di Torino

(Torino, 3 giugno 2019 e 12-13 novembre 2019)

(C) 2021

Accademia University Press

via Carlo Alberto 55

I-10123 Torino

\section{(c) (i) ()ㅜ $\Theta$ \\ BY NC ND}

prima edizione: luglio 2021

isbn 979-12-80136-43-5

edizioni digitali www.aAccademia.it/religioni_sviluppo_sostenibile

https://books.openedition.org/aaccademia/180

book design boffetta.com

Accademia University Press è un marchio registrato di proprietà di LEXIS Compagnia Editoriale in Torino srl 


\section{Introduzioni}

Agricoltura e Ambiente: una sfida complessa Amedeo Reyneri

La natura non spenta. Religioni e sostenibilità Oreste Aime

Le religioni e le nuove sfide di sviluppo sostenibile

\section{Ambiente e alimentazione} (obiettivi 2, 12 e 15 Agenda Onu 2030)

\section{I.1. Gli obiettivi dello sviluppo sostenibile}

Agricoltura sostenibile e biodiversità: le produzioni animali

Agricoltura sostenibile e biodiversità: le produzioni vegetali

Sostenibilità ambientale, sicurezza alimentare e politiche dell'Unione europea nel quadro degli obiettivi dell'Agenda 2030

Maria Pia Genesin

Consumo responsabile e lotta allo spreco

I.2. II contributo delle religioni

Elementi di sviluppo sostenibile nell'hinduismo Alberto Pelissero

II Creato: utilizzo consapevole di animali ed ambiente nell'Ebraismo

Gli obiettivi dello sviluppo sostenibile nell'Enciclica Laudato si'

II. Comunità umane solidali e inclusive (obiettivi 4, 10, 11 e 16 Agenda Onu 2030)

\section{II.1. Gli obiettivi dello sviluppo sostenibile}

II patrimonio culturale per le "città sostenibili" Cristina Videtta 143

Crescita economica e sviluppo solidale

II.2. II contributo delle religioni

Comunità umane, solidali, inclusive 
II dovere di istruire e di istruirsi

III. Vita umana, lavoro e sviluppo tecnologico

(obiettivi 3, 8 e 9 Agenda Onu 2030)

III.1. Gli obiettivi dello sviluppo sostenibile

Note critiche su innovazione tecnologica, lavoro e big data nella visione dell'agenda 2030. Obiettivi 3, 8, e 9 e capitalismo della sorveglianza

III.2. II contributo delle religioni

Vita umana, lavoro e sviluppo tecnologico (obiettivi 3,8 e 9 Agenda Onu 2030)

Vita umana, lavoro e sviluppo tecnologico (obiettivi 3, 8 e 9 Agenda Onu 2030):

il punto di vista del Cattolicesimo con un'appendice dopo covid 19

Gli autori 


\section{Presentazione}

Luca Battaglini, Ilaria Zuanazzi
aA

L'Agenda 2030 per lo sviluppo sostenibile è un programma d'azione sottoscritto nel settembre 2015 dai governi dei 193 Paesi membri delle Nazioni Unite. Il progetto ingloba 17 obiettivi per lo sviluppo sostenibile - Sustainable Development Goals, Sdgs - per un totale di 169 'target' o traguardi, compresi in cinque $\mathrm{P}$ : persone, pianeta, prosperità, pace e partnership. Per rilanciare e promuovere una mobilitazione generale di attuazione degli obiettivi, l'Alleanza italiana per lo Sviluppo Sostenibile (AsviS) ha indetto in tutta Italia il Festival dello Sviluppo Sostenibile, dal 21 maggio al 6 giugno 2019 e aderendo a questo progetto il Centro interdipartimentale di Ricerca in Scienze Religiose "Erik Peterson" dell'Università di Torino, insieme con altri centri di ricerca e Dipartimenti dell'Università di Torino (Centro di ricerca CRAFT, Dipartimento di Scienze Agrarie, Forestali e Alimentari, Dipartimento di Scienze Veterinarie, Dipartimento di Giurisprudenza) e con la Facoltà Teologica dell'Italia Settentrionale - sezione parallela di Torino, ha organizzato un ciclo di incontri dedicati al peculiare impulso che le Religioni possono offrire per il raggiungimento del progetto dell'Agenda Onu.

Quantunque la riflessione sull'importanza di perseguire gli obiettivi di sviluppo sostenibile si sia ampliata e poten- 
Religioni e sviluppo sostenibile

VIII ziata in epoca relativamente recente, da ben più tempo le tradizioni religiose nello sviluppo plurisecolare delle loro dottrine e prassi, ciascuna secondo il proprio peculiare ordine assiologico, promuovono i valori che presiedono alla cura del creato, alla dignità della persona, al bene comune. Numerose sono le attestazioni di questa attenzione che si sono incrementate negli ultimi anni, non solo nel magistero della Chiesa cattolica (Giovanni Paolo II, Centesimus annus; Benedetto XVI, Caritas in veritate; Francesco, Laudato si'), ma anche nei documenti promossi da altre confessioni religiose, specialmente sul tema della tutela dell'ambiente $(A$ Rabbinic Letter on Climate Crisis, 29 ottobre 2015; Bhumi Devi Ki Jai! A Hindu Declaration on Climate Change, 23 novembre 2015; Islamic Declaration on Global Climate Change, 18 agosto 2015; The Time to Act is Now: A Buddhist Declaration on Climate Change, 14 maggio 2015).

Il contributo che le religioni possono offrire agli obiettivi del millennio è stato di recente sottolineato nella conferenza internazionale Religioni e obiettivi di sviluppo sostenibile: ascoltare il grido della terra e dei poveri, promossa dal Dicastero per il servizio dello sviluppo umano integrale e dal Pontificio Consiglio per il dialogo interreligioso della Santa Sede (7-9 marzo 2019), che ha visto la partecipazione di numerose delegazioni di diverse confessioni religiose. Come è stato sottolineato in quella sede anche dal discorso rivolto ai partecipanti da papa Francesco, in un momento in cui l'impegno per la realizzazione degli obiettivi si è un po' raffreddato, le religioni possono cooperare fortemente per ritrovare l'entusiasmo iniziale e ridare nuovo impulso al lavoro comune. Non solo perché le religioni sono già coinvolte in tanti progetti di sviluppo in molti Paesi, ma soprattutto perché possono offrire motivazioni forti per una presa di coscienza seria e un coinvolgimento autentico, attingendo ai valori morali, sociali, giuridici e politici tratti dal loro patrimonio di dottrina e di tradizione.

Le religioni possono pertanto dare sostanza e sostegno etico e spirituale a un nuovo modello culturale di sviluppo integrale che non guardi solo agli obiettivi economici e non si affidi solo agli strumenti tecnologici, ma promuova nella complessità degli aspetti ecologici e antropologici, e quindi anche religiosi, il rispetto di tutti gli esseri viventi; lo sfruttamento responsabile delle risorse naturali; il con- 
solidamento di relazioni sociali improntate alla solidarietà, all'inclusione e alla promozione delle persone; l'impostazione dei rapporti produttivi e commerciali secondo modalità autenticamente umane.

Il programma del ciclo di incontri su "Religioni e sviluppo sostenibile" organizzato dall'Università di Torino era suddiviso in tre appuntamenti, che corrispondono alle tre parti del presente volume, dedicati ciascuno a un particolare ambito di azione: "Ambiente e alimentazione (obiettivi 2, 12 e 15 Agenda Onu 2030)"; "Comunità umane solidali e inclusive (obiettivi 4, 10, 11 e 16 Agenda Onu 2030)"; "Vita umana, lavoro e sviluppo tecnologico (obiettivi 3, 8 e 9 Agenda Onu 2030)". Per ogni settore sono stati individuati alcuni obiettivi strategici e su questi traguardi sono stati invitati a riflettere, in primo luogo gli esperti sotto il profilo scientifico, per illustrare il significato e i contenuti dei singoli obiettivi, e in secondo luogo gli studiosi o i rappresentanti delle diverse tradizioni religiose, per precisare il valore attribuito a questi obiettivi dalla dottrina confessionale e sottolineare il contributo che intende dare per la loro promozione.

L'interesse per gli approfondimenti contenuti nei diversi contributi e la vivacità del dibattito suscitato dal confronto interdisciplinare e interreligioso hanno convinto i promotori degli incontri a raccogliere nel presente volume le relazioni che, per disponibilità degli autori, è stato possibile pubblicare. L'ampiezza dei temi trattati, l'originalità del taglio di comparazione tra discipline scientifiche e prospettive religiose, oltre che lo sguardo olistico sulle molteplici problematiche che emerge dalla pluralità delle voci, possono rendere pienamente ragione dell'autorevolezza e dell'importanza del presente volume. 



\section{Introduzioni}

aA 

Nel sottoscrivere e promuovere i 17 obiettivi per lo sviluppo sostenibile - Sustainable Development Goals, Sdgs - la comunità internazionale ha assunto l'impegno di adottare e indirizzare politiche coerenti con lo sviluppo sostenibile.

In questo contesto il settore primario ed in particolare l'Agricoltura, nella sua accezione più ampia di attività agrosilvo-pastorale, svolge un ruolo chiave risultando coinvolta a titolo principale o secondario in ben 13 dei Sdgs. Per tale motivo l'attenzione verso questo settore è sensibilmente cresciuta, attraverso il riconoscimento del ruolo cruciale che esso svolge nei confronti della società non solo per la funzione chiave dell'alimentazione, ma più in generale dell'ambiente e dei servizi connessi. Peraltro, tale riconoscimento non è certamente nuovo dal momento che già le cronache del passato hanno riconosciuto l'influenza reciproca dell'attività agricola sui sistemi ecologici che poi, solo dagli albori del xIx secolo, abbiamo imparato a chiamare "ambiente". Con andamento spesso ciclico le società hanno sfruttato severamente le risorse naturali subendo le conseguenze degli squilibri, tanto da fare decadere intere civiltà un tempo potenti, quali quelle che hanno popolato la Mesopotamia o la più piccola Isola di Pasqua. Tuttavia a partire dal XviII secolo lo sfruttamento delle 
risorse ai fini agricoli ha subito un'accelerazione spinta dalla crescente domanda di prodotti, non solo alimentari, espressa da una popolazione sempre più numerosa ed esigente. Le due rivoluzioni agricole, la prima che ha diffuso i paradigmi dell'agricoltura intensiva a ciclo continuo e, successivamente, la seconda che nel xx secolo ha incrementato ancora di più le rese facendo ricorso in modo scientifico alle innovazioni offerte dalla genetica, dalla chimica e dalla meccanica, hanno permesso di sostenere fin qui e in modo straordinario la crescita della domanda; ciò è avvenuto in misura tale da offrire oggi risorse alimentari in misura pro capite superiori a quelle mai prima sperimentate dall'umanità. Più in dettaglio, questo risultato è stato ottenuto attraverso un triplice percorso: fino alla metà del secolo scorso con l'ampliamento delle superfici coltivate e a discapito degli ecosistemi naturali, quindi con l'aumento delle rese e, più recentemente, attraverso una rete logistica più capillare ed efficiente, tale da consentire una distribuzione geografica delle risorse alimentari più rispondente alla domanda. Questo percorso indubbiamente ha concorso in modo determinante a ridurre il drammatico verificarsi delle carestie e più in generale ad offrire le derrate alimentari di base, si pensi ad esempio ai cereali, alla carne e al latte, a costi reali sempre più ridotti e più accessibili a fasce crescenti della popolazione. Per perseguire questi risultati si è però aumentato lo sfruttamento del suolo e l'uso di risorse non rinnovabili, in particolare con l'impiego di fonti energetiche fossili, con riflessi importanti su biodiversità e cambiamenti climatici, oltre che sulla qualità dell'aria e dell'acqua.

Dov'è quindi la sfida che gli Sdgs rilanciano sull'Agricoltura? Rispondere alla domanda di beni e servizi che l'umanità reclama a fronte di un crescente rispetto delle risorse ambientali, cioè alimentare il mondo in un contesto di neutralità carbonica, di tutela della qualità ambientale e della biodiversità e di equità sociale. Ciò è quanto è stato espressamente delineato dalla Commissione dell'Unione Europea all'atto del suo insediamento nella primavera del 2020 e riassunto nel Green Deal Europeo attraverso i 2 Programmi strategici per la biodiversità e per l'alimentazione (From farm to fork).

Il problema è che questa è una sfida urgente che non possiamo perdere, ma per aumentare la probabilità di suc- 
cesso occorre che le diverse dimensioni del sapere scientifico, della cultura e delle differenti espressioni della religiosità siano in sintonia. La dimensione religiosa, seppure con diversa sensibilità tra le fedi, ha avuto un rilevante merito: quello di avere manifestato e comunicato con coraggio il significato profondo delle nostre azioni e la responsabilità verso la "casa comune" che grava sulle nostre spalle di cittadini del pianeta.

Con il crescere della sensibilità del rapporto tra agricoltura e ambiente e con il varo di politiche a favore della sostenibilità, si manifesta in modo più evidente il conflitto tra diversi approcci; ciò genera un rischio, quanto mai concreto, di ostacolare la loro attuazione. In tale senso alcuni errori debbono essere evitati: attribuire acriticamente alla natura un ruolo positivo e all'uomo un ruolo negativo bocciando così a priori ogni nuova introduzione tecnologica; scegliere modelli ispirati ad un passato mitizzato e forse mai esistito e che non può essere riproposto; dettare soluzioni generaliste dimenticando la grande variabilità nel mondo delle condizioni ambientali, economiche e sociali, con il rischio di proporre un modello agro-alimentare adatto solo ai paesi più ricchi.

Alle confessioni religiose si chiede quindi di contribuire in grande misura ad indirizzare questa sfida fornendo quel senso profondo e insostituibile di appartenenza, di comunione e soprattutto di forza per affermare nuovi modelli di sviluppo sostenibile. 


\section{La natura non spenta. Religioni e sostenibilità}

Oreste Aime

«Il mondo è carico della grandezza di Dio. / Fiammeggerà, come fulgore da percossa lamina; / s'addensa e ingrandisce, come gocciolio d'olio / franto. Perché dunque l'uomo ora non teme la sua verga? / Generazioni hanno calpestato, calpestato, calpestato; / e tutto è arso dal commercio; / offuscato, insozzato dalla fatica; / e porta lordume d'uomo e ha lezzo d'uomo: il suolo / è nudo ora, né sente piede, essendo calzato. // Ma non per questo la natura è spenta; / vive in fondo alle cose la frescura più cara; / e sebbene l'ultima luce dal nero occidente partisse / oh, il mattino, dall'orlo bruno d'oriente, sorge - / perché lo Spirito Santo sopra il curvo / mondo cova con caldo petto e con ah! luminose ali» ${ }^{1}$.

Nel 1877, quasi al culmine della prima rivoluzione industriale e dell'impero inglese, nell'epoca febbrile del Chrystal Palace alla prima esposizione mondiale di Londra, Gerald Manley Hopkins, nel suo breve poemetto La grandezza di Dio, descrive qualcosa di grave, ancora invisibile ai più. La sua denuncia può valere anche per tempi più antichi, ma 
se sullo sfondo proviamo a immaginare le città di Manchester con le ciminiere fumanti e di Liverpool con i suoi docks trafficati, possiamo comprendere meglio che cosa voglia dire che la terra, la creazione, è calpestata, sottoposta a devastazione a depredata: "tutto è arso dal commercio". Le immagini del tempo lo confermano proprio mentre vogliono celebrare la potenza dell'industria e del mercato ormai diventato mondiale. Al lamento s'aggiunge per contrasto una diversa descrizione di quella natura violata, di cui esplora nascoste profondità e altezze: essa non è spenta né destinata alla morte. La luce s'annuncia ad oriente in opposizione al nero occidente e un alito, lo Spirito di Dio, cova la creazione come all'inizio dei giorni.

È trascorso un secolo e mezzo dalla scrittura di questi versi (pubblicati solo più tardi) che vogliono celebrare la grandezza di Dio, nonostante la derelizione del mondo. È quanto ci viene chiesto anche oggi, quasi fosse un piccolo manifesto veggente e preveggente: una percezione acuta e profonda della realtà e di ogni realtà, dell'insieme e del particolare, grazie ad una parola precisa e libera; l'individuazione delle ferite e il presentimento della natura ancora nascosta e inviolata; il senso della grandezza di Dio e di quanto può fare il suo Spirito - nella varietà dell'esperienza religiosa.

\section{La metamorfosi del mondo e la rivoluzione antropologica} Per ponderare le analisi proposte in questo volume, Religioni e sviluppo sostenibile, è bene tenere presente la «rivoluzione antropologica» che sta caratterizzando la fase recente dell'Antropocene ${ }^{2}$. Una rivoluzione che può essere datata nel suo inizio tra il 1945 (la bomba atomica e l'energia nucleare - riguarda la fisica) e il 1953 (la doppia elica del

2. La nozione di rivoluzione è polisemica e ambigua. Lapplicazione all'antropologia è adottata in analogia con quella di «rivoluzione scientifica» coniata a suo tempo per indicare quanto avvenuto tra Copernico (1543) e Newton (1687). Allora fu il cosmo - il cielo e la terra - ad essere coinvolto, ora è l'uomo stesso. Tra allora e oggi sono pure avvenute le rivoluzioni politiche (americana e francese) e la rivoluzione industriale con tutte le loro conseguenze. Nella nozione sono dunque implicate sia la conoscenza sia soprattutto l'azione, il modo di considerare e trattare gli 'oggetti' via via presi in considerazione. L'Antropocene, da considerare come un'era geologica successiva all'Olocene, è un'altra nozione molto discussa; il suo inizio è solitamente datato con il 1800 (rivoluzione industriale); alcuni propongono il 16 luglio 1945 (bomba atomica). 
DNA, secondo James Watson e Francis Crick - tocca la biologia). Da quel momento il tradizionale ordine, che prevedeva la soggezione drammatica dell'uomo alla natura, si è capovolto definitivamente; a essere emblematicamente $\mathrm{e}$ concretamente messi in questione sono il pianeta Terra e la vita sul pianeta nel segno della possibile distruzione e della progressiva manipolazione. Nei decenni successivi con l'accelerazione economica e tecnica divenuta esponenziale, il pianeta, definitivamente colonizzato e "globalizzato", è stato esposto a un dominio sempre più insidioso e devastante, "calpestato" e "arso dal commercio". È ciò che oggi va sotto il nome di «rischio ecologico», ormai ben più di un rischio.

Non è però il solo pericolo. Bisogna aggiungervi il «rischio digitale» che incombe sulla vita mentale e associata dell'umanità che abita il pianeta: la rivoluzione informatica, aggiuntasi a quella fisica e biologica, nelle sue concrete applicazioni è un salto qualitativo e positivo nell'infosfera o il preludio a una sempre più estesa società del controllo?

La questione della sostenibilità si colloca entro questo ampio quadro e ne è uno degli elementi qualificanti. Sottolinearne la distinzione serve a non trasformare questo aspetto in uno schermo riduttivo o protettivo dell'altro; in forma quasi banale, si deve affermare che non è sempre e solo questione di sostenibilità, quasi questa si risolvesse unicamente in un controllo più accurato degli equilibri, prescindendo dal resto. Ulrick Beck ha lapidariamente ricordato che più la catastrofe si avvicina, meno la si vede ${ }^{3}$, talvolta a motivo degli stessi allarmi.

Per l'indagine sulla sostenibilità, infatti, occorre tener conto, almeno sullo sfondo, sia la «società del rischio» sia la «metamorfosi del mondo», sempre secondo le formule di Beck; a livello sintomatico, è altresì indispensabile prendere in carico le proposte post-umaniste e trans-umaniste perché esprimono lati possibili della«rivoluzione» antropologica per quanto in versioni estremamente discutibili ma in grado di attirare ingenti capitali e sperimentazioni liminari ${ }^{4}$. La sostenibilità, dunque, ha a che fare con il rischio ma anche con la possibile violazione senza consenso

3. U. Beck, La metamorfosi del mondo, Laterza, Roma-Bari 2017 (ed. or. 2016).

4. M. O'Connell, Essere una macchina, Adelphi, Milano 2018 (ed. or. 2017); Y. N. Harari, Homo Deus. Breve storia del futuro, Milano, Bompiani 2017 (ed. or. 2015). 
di limiti considerati invalicabili sia sul piano planetario che su quello dell'umanità.

\section{Basteranno i miglioramenti possibili?}

Se è corretta l'analisi e la sua sintetica interpretazione, si deve tener conto di una doppia sostenibilità, quella del pianeta e quella dell'umanità che la abita. Nel primo caso è questione di uso corretto e di ripartizione delle risorse, dell'incremento o della decrescita economica, del controllo delle conseguenze (consumi, sprechi, rifiuti ...), dell'impatto ambientale in rapporto alla più generale mutazione climatica; nel secondo si tratta di una ecologia della mente all'interno della rivoluzione antropologica detta (indotta da automatizzazione, infosfera, sperimentazione genetica, bioingegneria e dalle loro conseguenze sulla struttura fisica, psichica e sociale degli individui e delle collettività $)^{5}$.

Anche in questo caso occorre cogliere l'interdipendenza circolare delle due ecologie: un non corretto rapporto «mentale» tra uomo e natura fa sì che le soluzioni per la correzione dell'attuale pericolante ecosistema non siano trovate o siano facilmente eluse, grazie a un potente e collaudato negazionismo.

La mutazione del clima ha a che fare anche e forse soprattutto con le questioni sollevate dalla ecologia della mente che si dirama nella vita sociale e politica, anche se gli ambiti di solito siano considerati indipendenti. È, infatti, anche se non soprattutto una questione di paradigmi teorici e pratici. Bisogna ridiscutere lo schema oppositivo Natura/ Cultura (Latour); occorre, inoltre, contrastare la riduzione della mente al modello della macchina, fosse pure la più potente (Benassayag). Da questi modelli meramente oppositivi e riduzionisti dipende anche il modo di intendere l'azione umana. I modelli lineari di intervento, quelli stessi a salvaguardia del pianeta, progettati soltanto sullo schema causa-effetto non sono sufficienti; occorre delineare e adot-

5. L. Floridi, La quarta rivoluzione. Come l'infosfera sta trasformando il mondo, Cortina, Milano 2017 (ed. or. 2014); M. Benasayag, La tirannia dell'algoritmo, Vita e Pensiero, Milano 2020 (ed. or. 2019). Un percorso ancora più articolato è quello proposto da $\mathrm{B}$. Latour, La sfida di Gaia. Il nuovo regime climatico, Meltemi, Milano 2020 (ed. or. 2015). 
tare quelli intuiti dal pensiero della complessità che prevede circolarità, retroazione ${ }^{6}$.

Raggiungere questo livello paradigmatico più articolato è indispensabile, tuttavia non è ancora sufficiente; occorre intersecare un altro livello, che peraltro si fa già avvertire in quelli fin qui ricordati, quello etico-politico. L'elemento più importante e più sfuggente è quello degli "stili di vita" e si colloca come fattibilità al punto di incrocio delle due ecologie, del pianeta e della mente. Ci è di aiuto la proposta di Mark Hunyadi ${ }^{7}$. Oggi in occidente siamo protetti come non mai in quanto individui, ma questo avvento della cultura dei diritti individuali è la miglior garanzia per i sistemi strumentali - economici, finanziari, tecnico-scientifici - per dispiegare reti complesse alle quali si è impreparati. Di fatto ci rende estranei al mondo: «la vittoria dell'individuo segna in realtà la vittoria del sistema che ha creato l'individuo a propria immagine e a proprio vantaggio. Alla proclamata universalità dei diritti umani corrisponde l'effettiva globalizzazione di sistemi inumani» ${ }^{8}$.

Secondo Hunyadi occorre instaurare un'istituzione all'altezza della sfida, che sia il luogo di un agire comune orientato al mondo e non ai principi, da imporre in maniera volontaristica e con urgenza: un «Parlamento dei modi di vita» (così come si sono creati i comitati etici e con gli strumenti che l'evoluzione degli stessi modi di vita ci consegnano). Importante è mettersi alla «scuola del comune» - con immaginazione istituzionale: creare, inventare e tentare. Occorre un ordine indipendente dal fattuale. « $\grave{E}$ necessario ristabilire il primato del comune, a prezzo di chiedere alle libertà individuali di adeguarsi a questa nuova gerarchia di valori» ${ }^{9}$.

Lasciando qui in sospeso la fattibilità di questa proposta, c'è almeno da ricavare che oltre alla dimensione etica si pone un problema politico di entità immensa. Le nostre

6. M. Ceruti, Il tempo della complessità, Cortina, Milano 2018; M. Ceruti, F. Bellusci, Abitare la complessità. La sfida di un destino comune, Mimesis, Milano-Udine 2020.

7. M. Hunyadi, La tirannia dei modi di vita. Sul paradosso morale del nostro tempo, ETS, Pisa 2016 (ed. or. 2015).

8. Ivi, p. 56.

9. Ivi, p. 130. Dal punto di vista del «nuovo regime climatico», B. Latour, Tracciare la rotta. Come orientarsi in politica, Cortina, Milano 2018. 
strutture politiche, vincolate allo schema nazionale, con una distribuzione democratica del potere decisionale, sia a livello costituzionale sia a livello procedurale sono antiquate rispetto ai problemi che ci sovrastano e palesemente incapaci ad affrontare le questioni che ruotano attorno alla sostenibilità.

\section{Le religioni nella metamorfosi del mondo}

Anche le religioni si trovano coinvolte in ciò che abbiamo finora descritto. Nel mondo occidentale esse sono soggette ad un processo di post-secolarizzazione che accentua l'autonomia degli stili di vita e forme di de-istituzionalizzazione ${ }^{10}$; è difficile prevederne gli esiti anche in rapporto ai problemi posti dalla sostenibilità, che richiedono forte sensibilità per la «casa comune» e istituzioni nuove all'altezza dei rischi precedentemente elencati. Altrove permangono tratti più tradizionali ma anch'essi sottoposti a rilevanti trasformazioni. In ogni caso la funzione sociale delle religioni sta mutando ovunque, non senza conseguenze per il sentire e l'agire comuni.

Qual è, dunque, il contributo che le religioni possono dare alle questioni qui in discussione? Se diamo uno sguardo al passato, il sacro ha sempre investito e pervaso la realtà della natura e del mondo. Un antico tempio - egizio, greco, indiano, basilicale o romanico ... - trova la sua collocazione nel mondo senza violarlo: lo spazio sacro illumina lo spazio profano, e viceversa, evocando corrispondenze e limiti. Tuttavia, come molta ricerca antropologica ha messo in luce, il sacro è ambivalente; porta con sé la tentazione della violenza e potrebbe esserne un tentativo di soluzione, come ha mostrato il dibattito sull'opera di René Girard. A questo proposito, c’è da domandarsi quanto la logica sacrificale sia stata secolarizzata in tanti comportamenti definiti razionali che connotano la nostra società dei consumi, persino in una forma più estrema rispetto al (deprecato) passato ${ }^{11}$.

10. L. Berzano, Quarta secolarizzazione. Autonomia degli stili, Mimesis, Milano-Udine 2017; Id., Spiritualità. Moltiplicazione delle forme, Editrice Bibliografica, Milano 2017.

11. R. Girard, La violenza e il sacro, Adelphi, Milano 1980 (ed. or. 1972); Portando Clausewitz all'estremo. Conversazione con Benô̂t Chantre, Adelphi, Milano 2008 (ed. or. 2007); J.P- Dupuy, La marque du sacré, Carnets Nord, Paris 2008; R. Calasso, Linnominabile attuale, Adelphi, Milano 2017. 
Tenendo dunque conto dell'ambivalenza, sacrale antica e secolarizzata moderna, possiamo far tesoro di quanto David Tracy nelle sue lezioni lovaniensi ricordava: il mondo sacrale ancestrale, perduto o quasi, aveva un rapporto simpatetico con la natura, rapporto che non conosciamo né pratichiamo più ${ }^{12}$. Lo rievoca in alcune pagine numinose Willa Cather, quando nel romanzo La morte viene per l'arcivescovo (1927) descrive il viaggio di uno dei due protagonisti con una guida navajo verso le terre del New Mexico.

Sempre da Tracy possiamo riprendere uno spunto per ascoltare quanto hanno da dirci le tradizioni religiose diverse dalla nostra: "Rimani fedele alla tua tradizione, approfondisci continuamente le sue peculiarità, difendi e chiarisci la sua identità; $m a$, allo stesso tempo, vaga [...] in mezzo agli altri percorsi e tradizioni, tenta di apprendere qualcosa della loro bellezza e verità, concentrati sulla loro alterità e diversità, come nuova strada verso la comunanza». Assumendo questo atteggiamento, ma senza nascondere i nodi problematici, possiamo ricavare il contributo proprio a ciascuna religione e confessione e poi interagire nel dialogo ravvicinato o nella distanza, grazie alla volontà di ascolto che il recente dialogo interreligioso sta lentamente e faticosamente abbozzando.

Questo capitolo dedicato alla religione e alle religioni non è affatto pleonastico. L'importanza, persino l'insostituibilità, possono essere ricavate da alcune considerazioni della Laudato si' di papa Francesco. Nel quinto capitolo propone una traccia scientifica, etica, politica alla luce del dialogo come categoria unificante e volta a indirizzare concretamente l'elaborazione della cura della casa comune. A conclusione, quasi a coronamento, richiama la necessità del dialogo tra le scienze e le religioni: «qualunque soluzione tecnica che le scienze pretendano di apportare sarà impotente a risolvere i gravi problemi del mondo se l'umanità perde la sua rotta, se si dimenticano le grandi motivazioni che rendono possibile il vivere insieme, il sacrificio, la bontà» (n. 200).

A questo capitolo, indispensabile come abbiamo visto finora, se ne aggiunge un altro. Se la scienza e la politica non sono sufficienti per far fronte alla sfida ecologica nella 
sua ampiezza e gravità, solo attingendo a tutte le risorse disponibili sarà possibile reggere l'impegno; è qui che le religioni e le spiritualità possono dare il loro contributo, curando in particolare i processi educativi, a cui si dedica il sesto capitolo della stessa enciclica.

Una coscienza ecologica formata e consapevole con il corrispettivo stile di vita non s'improvvisano, richiedono un'attenta elaborazione, il riconoscimento di errori del passato e un collaudo prolungato nel tempo, aperto alla discussione e all'interazione. La novità del problema richiede alle religioni, e Francesco si concentra su quella cristiana, un loro rinnovato sforzo di approfondimento e di interpretazione, anche per diminuire piuttosto che aumentare il divario tra gli approcci scientifici (etici, politici) e le prospettive religiose. A questo scopo, non si tratta solo di ripetere dottrine consolidate ma di re-inventare (ritrovare in un modo nuovo) le proprie stesse fonti.

Grazie a questo intreccio ampio e complesso - l'ecologia della natura e della mente, gli stili di vita all'incrocio tra etica e politica, la scienza e la spiritualità, la religione e le religioni - la categoria di sostenibilità può esplicare tutta la sua potenzialità. Non è melting pot né sincretismo ma dialogo, in cui ci si arricchisce a vicenda, senza evitare le questioni brucianti che scaturiscono dalla cecità prospettica che si tramuta in violenza, anche quella quotidiana e abitudinaria, sulla natura e quindi sulle donne e sugli uomini che la abitano e che invece se ne debbono prendere cura, prima e al di là di ogni possesso. 
Dacci oggi il nostro bollettino quotidiano. È quello che da un anno a questa parte - ai tempi del Covid19 - sembra volersi imporre ad oggetto di meditazione mista a preghiera. Un uragano planetario ci ha progressivamente colti tutti di sorpresa, costringendoci a fermarci, a restare immobili. Mentre ancora assistiamo - sgomenti e nel contempo speranzosi in un miglioramento grazie ai vaccini - al conteggio quotidiano dei decessi e degli ammalati, siamo protesi a guardare al futuro con occhi diversi, siamo davanti ad un semaforo rosso, in attesa che cambi colore per la ripartenza.

E tuttavia l'arresto improvviso della frenetica vita quotidiana ha sortito i suoi aspetti positivi; ciascuno, a volte ripercorrendo le immagini della propria vita, ha potuto godere dei ritmi più lenti della natura, una natura che bene sa difendersi contro le ferite inferte dall'uomo, come assistiamo, nostro malgrado oggigiorno, con i nostri stessi occhi e sulla nostra stessa pelle.

Allora, quanto mai opportuni appaiono, nel frangente storico che ci occupa, i contributi di attenti studiosi che, analizzando le cause del degrado ambientale, ne suggeriscono le prospettive future, in un'ottica di sviluppo sostenibile, 
evidenziandone il nesso inscindibile e affatto trascurabile tra fattore religioso e ambiente; non è ultroneo sottolineare che l'elaborazione teorica non sia disgiunta dalla concreta attenzione di ciascuno alla casa comune: l'ecologia umana e l'ecologia ambientale camminano insieme (papa Francesco).

Trattasi di un percorso interdisciplinare teso alla ricerca di un'etica ambientale condivisa (correlata alla filosofia, alla teologia, all'economia), la quale concernendo ineludibilmente la relazione uomo -natura, involge tanto la sua dimensione spirituale, quanto le sue convinzioni religiose o filosofiche e riverbera nella coscienza ecologica per il bene comune.

Privilegiando il rapporto uomo-natura (che assume valore trascendente con riguardo alla dimensione religiosa) si può valorizzare la dimensione etica e la tutela dell'ambiente sia sul piano morale sia su quello fattuale.

Leconomia di profitto, che ha segnato i nostri tempi, ha prodotto riverberi dannosi sul pianeta; come non ricordare l'urgente questione dello smaltimento illecito dei rifiuti tossici e dei rifiuti pericolosi e il riconnesso, indifferibile obiettivo di contrastare tale abuso?

Certamente il fattore religioso assume un ruolo determinante nell'individuazione di comuni valori etico-religiosi a favore della tutela del Creato. Anzi, le religioni possono rappresentare un efficace veicolo attraverso cui far transitare valori comuni che possano condurre all'obiettivo della realizzazione di una comune coscienza etica.

L'analisi è molto interessante, a prescindere dall'ottica, biocentrica, ecocentrica o antropocentrica dalla quale ci si pone. Ben possono, infatti, rinvenirsi valori comuni sia abbracciando una visione antropocentrica, tipica delle religioni rivelate, che considera l'uomo al centro del Creato (seppure ridimensionandone l'antica concezione di dominatore indiscusso) e che attribuisce priorità all'essere umano rispetto alle altre specie viventi, per cui l'uomo è chiamato a servirsi delle cose del mondo nella consapevolezza che non gli appartengono; sia nell'ottica di una visione biocentrica, fondata sulla convinzione e sulla consapevolezza che la sopravvivenza della specie umana dipenda dalla sopravvivenza delle altre specie, in quanto ognuna è legata all'altra in un insieme armonico, in relazione biunivoca di interdipendenza, sia ancora nell'ottica ecocentrica, in cui 
assume priorità la salvaguardia delle specie e degli ecosistemi rispetto a quella dei singoli appartenenti ad essi e ciò vale anche per gli esseri umani.

In ogni caso si tratta di favorire una nuova dispositio animi nei confronti dell'ambiente naturale che si pone in alternativa ad una visione indifferentista o utilitaristica.

La ricerca di un'etica ambientale condivisa è indissolubilmente riconnessa ad una valorizzazione del connubio uomo-natura, che coinvolge anche la sfera spirituale e le convinzioni religiose. Solo in questo modo potrà essere rivalutata la dimensione etica e la conseguente tutela che tale nesso merita anche sul piano morale.

Non meraviglia pertanto che le religioni abbiano un comune denominatore: pur nella differenza delle rispettive posizioni, sono concordi nel considerare la giustizia, la carità, la libertà, la solidarietà in correlazione alla tutela dell'ambiente. Pur appartenendo ad epoche differenti Gandhi e papa Francesco sono concordi nell'affermare che il sogno di un pianeta migliore può realizzarsi soltanto quando si comincia a migliorare sé stessi.

Il dialogo ecumenico sulla ricerca scientifica e le conseguenze delle sue applicazioni sull'ambiente, possono condurre a scelte consapevoli e condivise nell'ottica di uno sviluppo sostenibile. Papa Francesco ammonisce: non si tratta solo di una questione che tocca la sfera dell'economia, ma anche l'etica e l'antropologia. Per questa ragione Egli riconnette il problema ambientale a quello della povertà, oltre che dell'indifferenza comune.

La visione islamica del Khalifa (vicereggente di Dio, al quale Allah ha affidato il compito di custodire e non defraudare la terra), la tradizione Vaishnava, il concetto di Karma e di Deep Ecology, oppure quello dell'ahimsa conducono all'opportunità di una maggiore coscienza ecologica verso una vera e propria conversione ecologica, attraverso la tolleranza verso le altre fedi, tutte fonte di arricchimento e di insegnamento. La stessa compassione nei confronti degli animali, insieme alla consapevolezza che l'azione buona ne genera un'altra altrettanto buona sono concetti condivisi dalla religione ebraica come da quella buddista.

Non è chi non veda, allora, come l'etica ambientale sia strettamente connaturata all'uomo inteso nella sua totalità, inclusa la sfera spirituale. 
Anzi, la sfida dei nostri giorni consiste nel saper cole nuove sfide niugare l'innovazione ecologica anche con il valore della trascendenza, che ritengo sommessamente irrinunciabile, soprattutto nel riuscire a trovare le regole generali di comportamento per il bene comune.

Loperosa convergenza per il bene comune tra Stato e comunità religiose potrà rappresentare il giusto approccio della trasformazione delle coscienze, presupposto per l'elaborazione di norme sulla salvaguardia dell'ambiente e sullo sviluppo sostenibile.

La collaborazione in uno stato democratico, che ancor più può estrinsecarsi all'interno di comunità umane, solidali, inclusive, anche a carattere religioso, presuppone un'etica forte e condivisa che renda maggiormente consapevoli e responsabili coloro che sono chiamati a partecipare alle scelte per la realizzazione del bene comune, anche attraverso l'utilizzo responsabile delle nuove tecnologie allo scopo di assicurare lavoro e benessere per l'umanità, attraverso una crescita economica sostenibile, nel rispetto dell'ambiente, nei settori più vari, dall'agricoltura all'allevamento, alla biodiversità, alla sicurezza alimentare e alla sostenibilità ambientale, verso un consumo più responsabile teso ad evitare lo spreco.

Ci troviamo di fronte a nuovi orizzonti di ruolo pubblico per gli attori religiosi. 

I. Ambiente e alimentazione

(obiettivi 2, 12 e 15 Agenda Onu 2030)

aA 

I.1. Gli obiettivi dello sviluppo sostenibile

aA 



\section{Agricoltura sostenibile e biodiversità: le produzioni animali \\ Luca Battaglini}

Larticolo 25 della Dichiarazione Universale dei Diritti Umani proclamata dall'Assemblea Generale delle Nazioni Unite ${ }^{1}$ sancisce il diritto all'alimentazione (right to food). Esso viene meglio definito nella decima risoluzione della dichiarazione Onu del 2000:

the right of everyone to have access to safe and nutritious food, consistent with the right to adequate food and the fundamental right of everyone to be free from hunger so as to be able fully to develop and maintain their physical and mental capacities. ${ }^{2}$

Come veniva specificato dal World Food Summit della Fao ${ }^{3}$, il concetto include tre pilastri: la disponibilità di cibo, l'accesso al cibo e le modalità di fruizione, in maniera appro-

1. Un (United Nations), 1948. The Universal Declaration of Human Rights. New York, USA. Disponibile a: http: //www.un.org/en/documents/udhr/index.shtml.

2. Un (United Nations) (2000). The right to food - Commission on Human Rights resolution 2000/10. Office of the High Commissioner for Human Rights, United Nations. Ginevra, Svizzera.

3. Fao (1996) Rome declaration on world food security. World Food Summit, Rome, 13-17 November 1996. Fao, Roma. 
priata, seguendo le conoscenze nutrizionali e sanitarie di base, nonché disponendo di acqua e di igiene adeguata.

L'Agenda 2030 per lo Sviluppo Sostenibile, programma d'azione per le persone, il pianeta e la prosperità, siglato nel settembre 2015 dai governi dei 193 Paesi membri dell'Onu, riprende tali esigenze inglobandole nei 17 Obiettivi per lo Sviluppo Sostenibile: i cosiddetti Sustainable Development Goals o Sdgs ${ }^{4}$. L'Agenda 2030 è un imponente programma d'azione per un totale di 169 target o traguardi. Dal 2016 in avanti gli Obiettivi per lo Sviluppo Sostenibile stanno vincolando i diversi Paesi: i diversi target dovranno essere raggiunti entro il 2030. Gli Obiettivi rappresentano finalità comuni su questioni fondamentali per lo sviluppo. Tra questi la lotta alla povertà e il contrasto al cambiamento climatico. Il secondo Goal intende porre fine alla fame e alla malnutrizione, per raggiungere la sicurezza alimentare e allo stesso tempo migliorare la nutrizione e promuovere un'agricoltura sostenibile. In considerazione di Agenda 2030, ma volendo andare oltre, la popolazione mondiale nel 2050 supererà i 9 miliardi di individui e si prevede che, grazie ad un relativo aumento del benessere, auspicabilmente nel maggior numero possibile di aree del pianeta, la composizione della dieta si orienterà sempre più verso i prodotti di origine animale. È risaputo che le produzioni degli animali d'allevamento hanno rappresentato nella storia dell'umanità la base per una corretta alimentazione, a prescindere da età, condizioni di salute e credo religioso. Come conseguenza di questa importante variazione prevista nei consumi e nelle relative produzioni vi è preoccupazione per gli impatti ambientali che gli attuali e nuovi allevamenti determineranno. Si renderà così necessaria una strategia globale a lungo termine che, per molti studiosi di settore, sarà realizzabile solo attraverso la cosiddetta intensificazione sostenibile dei sistemi zootecnici mondiali ${ }^{5}$.

\section{Impatto ambientale degli allevamenti: quali responsabilità}

La crescente consapevolezza dell'interazione dei sistemi

4. Un (United Nations) (2015). Development Programme. Sustainable Development Goals (Sdgs), New York, Usa.

5. Fao (2011) Save and Grow. A policy maker's guide to the sustainable intensification of smallholder crop production Fao, Roma. 
Agricoltura sostenibile e

biodiversità produttivi degli allevamenti con gli ecosistemi che li comprendono, sollecita l'attenzione di governi nazionali, organizzazioni sovranazionali e istituzioni culturali, sulla necessità di affrontare lo sviluppo agricolo, dunque anche quello dell'allevamento, in termini di sostenibilità ambientale. La necessità di utilizzare le risorse nel rispetto del territorio o dell'ambiente di produzione desta, a livello mondiale, una particolare attenzione: il tema dell'eticità del sistema produttivo s'interseca così con quello della sostenibilità ambientale e alimentare. Si può richiamare a tale proposito la richiesta del numero 2 degli Sdgs: «Porre fine alla fame, realizzare la sicurezza alimentare e una migliore nutrizione e promuovere l'agricoltura sostenibile».

Nei diversi rapporti della $\mathrm{Fao}^{6}$, le attività di allevamento sono presentate quali una delle principali cause dei problemi ambientali del pianeta. Esse vengono ritenute decisive nel portare all'aumento delle emissioni di gas-serra (Ghg o greenhouse gases), al degrado del suolo e all'inquinamento delle acque e di entrare in relazione con il cambiamento climatico (come la recente messa in relazione con la pandemia da Covid-19) ${ }^{7}$. In tal modo esse ricevono ampio risalto sollecitando la ricerca di possibili soluzioni nelle diverse Conferenze annuali sul Clima o Cop dell'Unfccc o Convenzione quadro delle Nazioni Unite sui cambiamenti climatici ${ }^{8}$.

Il settore dei prodotti di origine animale, e quello della carne con particolare evidenza, viene spesso richiamato per tali responsabilità. Si pensi ad esempio alla water footprint

6. H. Steinfeld, P. Gerber, T. Wassenaar, V. Castel, M. Rosales, and C. de Haan, Livestock's Long Shadow: environmental issues and options, Food and Agriculture Organization (FAO), Rome 2006, p. 390; H. Steinfeld, H.A. Mooney, F. Schneider, L.E. Neville, Livestock in a changing landscape: Drivers, consequences, and responses, Island Press, XIV, Washington DC 2010, p. 585; N. Alexandratos, J. Bruinsma, World agriculture towards 2030/2050: the 2012 revision, ESA Working paper No. 12-03, FAO, Roma 2012.

7. L. Spinney, Is factory farming to blame for coronavirus?, The Guardian, 2020, in https:// www.theguardian.com/world/2020/mar/28/is-factory-farming-to-blame-for-coronavirus.

8. Ipcc (Intergovernmental Panel on Climate Change), 2019. Global warming of $1.5^{\circ} \mathrm{C}$. An IPCC Special Report on the impacts of global warming of $1.5^{\circ}$, C. Masson-V. Delmotte, P. Zhai, H.-O. Pörtner, D. Roberts, J. Skea, P.R. Shukla, A. Pirani, W. Moufouma-Okia, C. Péan, R. Pidcock, S. Connors, J.B.R. Matthews, Y. Chen, X. Zhou, M.I. Gomis, E. Lonnoy, T. Maycock,, M. Tignor, and T. Waterfield (eds.) in https://www.ipcc.ch/site/assets/uploads/sites/2/2019/06/SR15_Full_Report_High_Res.pdf; P.J. Gerber, H. Steinfeld, B. Henderson, A. Mottet, C. Opio, J. Dijkman, A. Falcucci, G. Tempio, Tackling climate change through livestock - A global assessment of emissions and mitigation opportunities, FAO, Roma 2013. 
o impronta idrica che richiama il considerevole consumo di acqua per la sua produzione. Nel contempo molti sono i riferimenti agli effetti negativi sulla salute umana quale conseguenza del consumo di questi prodotti di origine animale. Ciò ha portato negli anni, anche attraverso misure legislative a protezione dell'ambiente, a significative modifiche nel comportamento alimentare di molti consumatori, con orientamenti più o meno evidenti nella limitazione, fino all'esclusione dalla dieta, di questa derrata. È dunque evidente come il tema dell'impatto delle produzioni degli allevamenti sull'ambiente e sulla salute umana appaia tra quelli di maggiore rilevanza, anche per le espressioni di responsabilità "etica" 9 .

Come conseguenza, il tema della compatibilità ambientale di queste attività risulta di fondamentale importanza per il riconoscimento di un suo valore anche in chiave "morale". Si può affermare che la sostenibilità ambientale nel produrre un alimento di origine animale sia anche base di una sua giustificazione "etica". Per conseguirla è innanzitutto necessario conservare l'ambiente per le sue funzioni principali, quale fonte di risorse naturali e fornitore delle condizioni necessarie al mantenimento della vita in senso ampio. È in buona sostanza fondamentale che le risorse rinnovabili non debbano essere sfruttate oltre la loro naturale capacità di rigenerazione; inoltre, la produzione di rifiuti ed il rilascio dei medesimi nell'ambiente devono sempre procedere a ritmi uguali o inferiori a quelli di una dimostrata e controllata capacità di assimilazione da parte dell'ambiente stesso e, nel contempo, devono essere assicurati i molteplici servizi di sostegno all'ambiente (ad es. mantenimento della biodiversità animale, riduzione delle emissioni, conservazione della qualità delle risorse naturali $[\ldots])^{10}$.

Occorre pertanto difendere l'integrità funzionale del sistema di allevamento entro il quale il prodotto è ottenuto adottando un criterio interdisciplinare per evitare che

9. G. Bertoni, Etica e allevamento animale, Franco Angeli, Milano 2017, p. 226.

10. L. Battaglini, Ecologia e sviluppo sostenibile. Sviluppo ecosostenibile e risorse alimentari: risvolti bioetici, in Dalla parte della vita. Società complessa e fragilità. La prospettiva della bioetica, Studia Taurinensia - Michele Pellegrino, Effatà, 2017, pp. 577-592. 
Agricoltura sostenibile e

biodiversità

le risorse rinnovabili siano sfruttate oltre la loro naturale capacità di rigenerazione ("impronta ecologica globale") ${ }^{11}$.

2. Verso una valutazione più equilibrata della sostenibilità nelle produzioni degli allevamenti

Sempre negli Sdgs si indica di raddoppiare entro il 2030, la produttività agricola e il reddito dei produttori di alimenti su piccola scala, in particolare donne, popolazioni indigene, famiglie di agricoltori, pastori e pescatori, consentendo un accesso sicuro e giusto alla terra e alle altre risorse. Sono peraltro necessari stimoli produttivi alla conoscenza, ai servizi finanziari, ai mercati e alle diverse opportunità creare valore aggiunto a questi prodotti (2.3 degli Sdgs). Come raggiungere tali obiettivi? Garantendo sistemi di produzione alimentare sostenibili, applicando pratiche agricole resilienti che aumentino la produttività e la produzione, conservando gli ecosistemi, rafforzando la capacità di adattamento ai cambiamenti climatici, alle condizioni meteorologiche estreme, alla siccità, alle inondazioni e agli altri disastri, anche per migliorare il terreno e la qualità del suolo (2.4 degli Sdgs).

Proprio gli allevamenti - come già si è detto fonte di alimenti di origine animale fondamentali per sostenere una corretta alimentazione nel mondo - vengono ritenuti tra $\mathrm{i}$ principali responsabili dei gas serra. A questo punto è lecito domandarsi: è possibile una riduzione delle emissioni di questo settore delle produzioni agricole? Le numerose iniziative di sviluppo e ricerca al proposito presentano soluzioni che si riferiscono all'adozione di appropriate tecniche: sistemi di alimentazione animale sostenibili, applicazione di buone pratiche derivanti da un generalizzato progresso sanitario e corretta gestione dei reflui in allevamento. E pertanto necessario indicare adeguate metodologie per le valutazioni dei danni ecologici, elencando e quantificando con precisione $\mathrm{i}$ problemi per ipotizzare opportune soluzioni.

Ferme restando le considerazioni preoccupanti sui danni ecologici recati dagli allevamenti, che restano tuttora difficili da definire con affidabilità, è doveroso contrapporre al- 
cune ragioni a sostegno di questa attività produttiva (tabella 1). Alcune espressioni dell'allevamento possono in effetti rivelarsi opportunità a favore dell'ambiente, con evidenti risvolti etici: attualmente ci si riferisce ai cosiddetti servizi "ecosistemici"12. Tra questi la difesa della biodiversità, la protezione e regolazione del territorio e degli habitat, gli aspetti culturali e sociali connessi, ecc. (tabella 2). A tale proposito può essere utile richiamare il ruolo che rivestono i sistemi agro-zootecnici estensivi o semi-estensivi basati prevalentemente sull'impiego di risorse spontanee. Si pensi appunto all'importante contributo dei sistemi pastorali per le produzioni degli allevamenti che si basano esclusivamente sull'impiego di risorse non altrimenti utilizzabili (greggi transumanti, mandrie da latte o di vacche nutrici di vitelli, allevamenti estensivi di suini e molte altre tipologie).

Lapplicazione di questi sistemi avrebbe inoltre favorevoli ricadute sulle proprietà nutrizionali dei prodotti, dal latte alle carni, e dei loro derivati (come, ad esempio, il naturale arricchimento dei formaggi e delle carni con acidi grassi, come i CLA, caratterizzati da preziose proprietà nutraceutiche $)^{13}$. Le funzioni a favore dell'ambiente sono pertanto numerose, a beneficio di una qualità anche di natura "etica". Questo con espressioni molteplici, dalla conservazione del paesaggio zootecnico (attraverso il mantenimento di ambienti pastorali “ordinati”) all'arricchimento in biodiversità (come la conservazione di razze autoctone con elevate doti di "resilienza" ambientale).

\section{La biodiversità animale e la sostenibilità dei sistemi: quali} opportunità?

Lobiettivo 2 degli Sdgs (2.5) è appunto quello di assicurare la diversità genetica di semi, piante coltivate e animali da allevamento e domestici e le loro specie selvatiche affini, anche attraverso banche del seme e delle piante gestite e diversificate a livello nazionale, regionale e internazionale. Ciò avviene promuovendo l'accesso e la giusta ed equa

12. Millenium Ecosystem Assessment, Ecosystems and human well-being: synthesis, Island Press, Washington DC 2005.

13. CLA o Acido linoleico coniugato con proprietà "nutraceutiche" (neologismo tra "nutrizione" e "farmaceutica" che si riferisce a proprietà degli alimenti benefiche sulla salute umana). 
Agricoltura sostenibile e

biodiversità

condivisione dei benefici derivanti dall'utilizzo delle risorse genetiche e delle conoscenze tradizionali collegate. Al punto 5.2 del medesimo obiettivo 2 vi è il richiamo alla necessità di operare investimenti in infrastrutture rurali, servizi di ricerca e di divulgazione agricola, ma anche allo sviluppo tecnologico e alle banche genetiche di piante e bestiame, al fine di migliorare la capacità produttiva agricola nei Paesi meno sviluppati.

Di particolare interesse sono i sistemi produttivi integrati tra colture e allevamento, praticati in prevalenza da piccoli agricoltori-allevatori. Questi sono assai diffusi in contesti limitatamente sviluppati o in aree meno favorite e consentono con maggiore evidenza ricadute in termini ecologici.

Queste iniziative di sviluppo incontrano le indicazioni del Goal 12 che intende garantire modelli sostenibili di produzione e consumo. A tale proposito si intende adottare entro il 2030 sistemi di gestione sostenibile per un uso efficiente delle risorse naturali. Ciò anche per ridurre lo spreco pro capite globale (dai rifiuti alimentari nella vendita e consumo fino alle perdite di alimenti nella filiera di produzione). Questo deve passare dalla consapevolezza del concetto di sviluppo sostenibile e dall'adozione di stili di vita il più possibile armonizzati con la natura. Un'attenzione particolare deve essere dedicata ai Paesi in via di sviluppo, perché anch'essi raggiungano modelli più sostenibili di consumo e di produzione. Sarà inoltre necessaria la promozione di un turismo sostenibile in grado di comprendere e promuovere la cultura e i prodotti locali.

A tale proposito le già richiamate attività pastorali, se convenientemente gestite, saranno in grado di rivestire queste importanti funzioni ecologiche. Sistemi in grado di assicurare un più conveniente valore, anche "etico", al prodotto, a sostegno della biodiversità animale e vegetale. Le sinergie tra coltivazioni, attività pastorali e produzioni agroforestali favoriscono la sostenibilità economica ed ecologica dell'allevamento. Si tratta pertanto di favorire "flussi" di servizi con valore ecosistemico.

Sistemi più estensivi sarebbero in grado di fornire prodotti diversificati: oltre a carne, latte, e derivati, anche fibre naturali come la lana, con evidenti vantaggi grazie all'origine "naturale" del prodotto. Anche qui il miglioramento delle tecniche gestionali unitamente alle attenzioni 
alla genetica degli animali (selezione) e all'alimentazione (in prevalenza a base di risorse naturali non impiegabili diversamente) potrebbe avere un valore non solo ambientale. Ladozione di opportune tecniche di pascolamento, fondamentali per la gestione anche di mandrie e greggi da carne, potrebbe consentire di ottimizzare la produzione evitando dissesti ambientali per eccessivo uso delle risorse (concentrazione di animali sui pascoli) o al contrario il degrado e la banalizzazione della risorsa pastorale per effetto dell'abbandono.

Attraverso questa maggiore biodiversità, l'efficiente riciclo dei nutrienti, la migliore salute del suolo e la conservazione delle risorse forestali, questi sistemi consentirebbero di aumentare la resilienza ambientale contribuendo all'adattamento al cambiamento climatico.

Occorre infine ricordare la necessità di una distribuzione socialmente retta di costi e benefici derivanti dal modo con cui l'uomo gestisce l'ambiente e la sua biodiversità. Questo diventa possibile attraverso una diversificazione e integrazione di risorse umane, socioculturali ed economiche. Il tutto deve infine fondarsi su un elevato grado di equità e giustizia sociale, di identità culturale, di coesione sociale e di partecipazione alle scelte e all'assunzione di responsabilità, per assicurare un valore "integrale" a queste produzioni.

\section{Ulteriori considerazioni}

Quali soluzioni si possono intravvedere? Come si è detto le sfide sono rappresentate dal raggiungimento di produzioni di alimenti di origine animale in adeguata quantità. Da un lato è necessario gestire correttamente le superfici agricole ad elevata vocazione produttiva, dall'altro riconsiderare superfici meno vocate all'agricoltura intensiva, ma di grande pregio ecologico, per salvaguardarne la biodiversità. Occorrerà puntare su un equilibrato aumento delle rese delle produzioni vegetali e animali, specialmente nei Paesi emergenti, tramite l'adozione di sistemi produttivi più efficienti e sostenibili, conciliando le attività agricole con le altre (produzione di energia, uso delle risorse idriche, sviluppo dell'urbanizzazione, attività industriali).

L'obiettivo è un equilibrio, assai complesso da raggiungere, tra l'aumento della produzione di alimenti e la salvaguardia delle risorse naturali. Si può pertanto parlare non 
Agricoltura sostenibile e

biodiversità

solo di intensificazione sostenibile ma anche di necessità di un ritorno a sistemi più estensivi di aree marginali e interne.

I modelli produttivi che rispondono a criteri di ricchezza di servizi ecosistemici richiedono una favorevole integrazione tra colture e allevamenti. Si tratta di sistemi praticati in prevalenza da piccole aziende di agricoltori e allevatori, assai diffusi in contesti meno avvantaggiati. Aree che oggi noi definiamo "marginali" e "interne" e che, purtroppo, per ragioni economiche e per scarsa dotazione di servizi, sono state in larga parte abbandonate. Un coerente ed equilibrato ritorno a questi territori consentirebbe benefiche ricadute in termini ecologici, con risvolti positivi in termini di etica ambientale. Anche Francesco, nella Laudato $s i^{14}$, aveva sviluppato questo tema (n. 129) «Vi è una grande varietà di sistemi alimentari agricoli e di piccola scala che continua a nutrire la maggior parte della popolazione mondiale, utilizzando una porzione ridotta del territorio e dell'acqua e producendo meno rifiuti, sia in piccoli appezzamenti agricoli e orti, sia nella caccia e nella raccolta di prodotti boschivi, sia nella pesca artigianale».

Queste espressioni si ritrovano nel quindicesimo Sdgs: «proteggere, ripristinare e favorire un uso sostenibile dell'ecosistema terrestre, gestendo coerentemente le foreste, contrastando la desertificazione, arrestando e facendo retrocedere il degrado del terreno, fermando la perdita di diversità biologica». Al punto 15.4 si riporta che «entro il 2030 occorrerà garantire la conservazione degli ecosistemi montani, compresa la loro biodiversità, al fine di migliorare la loro capacità di fornire prestazioni che sono essenziali per lo sviluppo sostenibile».

Una corretta integrazione dei sistemi di allevamento con quelli agricoli contribuirebbe efficacemente al miglioramento del bilancio globale delle risorse, incluso il carbonio per le implicazioni sui gas serra, non solo in termini di riduzione dei consumi energetici e idrici, ma anche, in relazione al grado di impronta ecologica. D’altro canto, si può anche ritenere che, in direzione totalmente opposta e con ulteriori 

dei cicli produttivi con l'aumento dell'efficienza dei sistemi agro-zootecnici ed il miglioramento della loro sostenibilità ambientale (il già richiamato concetto di "intensificazione sostenibile") coadiuvata da opportuni indirizzi di selezione genetica animale, si possa arrivare ad una sostanziale riduzione degli impatti, principalmente per effetto della diluizione dei medesimi riferita all'unità di prodotto ${ }^{15}$.

Il concetto di sostenibilità, convenientemente declinato, potrà aiutare il consumatore nelle sue scelte, nonché aiutare gli agricoltori a presentare i propri progressi in un modo più comprensibile. Un sicuro beneficio, in termini di sostenibilità dei sistemi produttivi, deriverà dall'azione dei decisori, all'interno o all'esterno del comparto produttivo agricolo, nel combinare le preoccupazioni sulla salute umana, il benessere animale e la conservazione delle risorse genetiche, in una prospettiva unitaria ${ }^{16}$.

\section{Conclusioni}

Un allevamento animale "sostenibile" (ed "etico") dovrà prevedere un impiego equilibrato del territorio e delle risorse con convenienti equilibri tra produzioni animali e vegetali, rispettando le caratteristiche ambientali e consentire la riproducibilità del sistema produttivo nel lungo periodo. Queste attività che continuano a rappresentare per l'umanità fonte di molteplici opportunità produttive e non solo, potranno ricevere oggi una piena giustificazione solo se attuate attraverso un corretto impiego delle risorse. Rimane altresì inscindibile da tutto ciò l'azione di formazione e di educazione alimentare e alla salute, al fine di migliorare le condizioni di nutrizione e di vita della popolazione mondiale, riducendo da una parte gli eccessi di alimenti e dall'altra stimolandone un consumo consapevole, responsabile ed eticamente sostenibile.

15. H.C.J. Godfray, J.R. Beddington, I.R. Crute, L. Haddad, D. Lawrence, J.F. Muir, J. Pretty, S. Robinson, S.M. Thomas, C. Toulmin, Food security: the challenge of feeding 9 billion people, Science, 2010, n. 327, pp. 812-818.

16. C. Gamborg, P. Sandøe, Sustainability in Farm Animal Breeding: A Review Livestock Production Science, Volume 92, 2005, Issue 3, pp. 221-231; P. Sandøe, Re-thinking the ethics of intensification for animal agriculture, in P.B. Thompson, The ethics of intensification: agricultural development and cultural change, The international library of environmental, agricultural and food ethics, 2008, no. 16, Springer Science Business Media B.V., pp. 191-198. 
Agricoltura sostenibile e

biodiversità

Ancora, Francesco, nella Laudato $s i^{\prime 17}$ richiama tali aspetti: la produzione di alimenti non deve essere vista soltanto come atto agricolo, ma anche ecologico. Sempre nell'Enciclica: «l'essere umano [può] intervenire nel mondo vegetale e animale e servirsene quando è necessario alla sua vita». Le problematiche relative all'impoverimento della natura e alla sua biodiversità, al cambiamento del clima, all'inquinamento in genere, mettono in evidenza le criticità delle politiche agricole ed i connessi e discutibili modelli produttivi e distributivi. Anche Agenda 2030, nel suo programma d'azione per lo sviluppo sostenibile, ci ricorda, nei suoi diversi obiettivi, il complesso gioco di interdipendenze, interne ed esterne all'ambiente, invitando l'uomo ad uscire dalla pretesa scientifica di poter dare un proprio ordine alla natura.

Tabella 1 - Espressioni positive e negative dei sistemi agro-zootecnici

Aspetti positivi

Diversificazione dei sistemi agricoli (ambienti pastorali, prati permanenti,...) e stimolo alle rotazioni agronomiche

Mantenimento della biodiversità (anche zootecnica attraverso la protezione delle diverse razze locali a limitata diffusione ed in particolare quelle a duplice attitudine)

Riciclo efficace degli elementi nutritivi e miglioramento della struttura del terreno agrario

Impiego di risorse foraggere spontanee per la produzione di alimenti, anche carni, di elevato valore nutrizionale e nutraceutico

Forme di manutenzione del territorio e del paesaggio
Impatti negativi

Desertificazione del suolo

Inquinamento e consumo delle acque

Erosione e inquinamento del suolo

Emissioni in atmosfera

Perdita di biodiversità

17. Francesco, Lettera enciclica "Laudato si" del Santo Padre Francesco sulla cura della casa comune, Libera Editrice Vaticana, Città del Vaticano 2015. 
Luca

Battaglini
Tabella 2 - Funzioni

\begin{tabular}{|c|c|c|}
\hline Funzioni & & Beni e servizi \\
\hline \multirow{3}{*}{ Produttiva } & Prodotti zootecnici & Alimenti di origine animale \\
\hline & Flora e fauna & Biodiversità animale e vegetale \\
\hline & Climatica & $\begin{array}{l}\text { Fissazione del carbonio, regolazione } \\
\text { di gas serra }\end{array}$ \\
\hline \multirow[t]{3}{*}{ Regolatrice } & Rischi naturali & Protezione da frane, inondazioni \\
\hline & Prevenzione erosione & Mantenimento dei suoli \\
\hline & Controllo biologico & Controllo di specie invasive \\
\hline Conservatrice & & Protezione degli habitat \\
\hline \multirow{3}{*}{$\begin{array}{l}\text { Culturale e } \\
\text { ricreativa }\end{array}$} & Culturali & Eredità culturale, linguistica \\
\hline & Artistici & Architettura, opere artistiche \\
\hline & Aspetti estetici & Turismo \\
\hline
\end{tabular}


L'agricoltura e i sistemi alimentari sono tra i principali motori dei cambiamenti globali sulla Terra e si collocano al centro dei sistemi socio-economici, esito dell'Antropocene ${ }^{1}$. Sin dalle sue origini, l'agricoltura ha generato cambiamenti più o meno repentini sul paesaggio, nell'uso delle risorse naturali e sulla biodiversità vegetale e animale ${ }^{2}$. In particolare le innovazioni introdotte nel periodo industriale, iniziato intorno al 1800, hanno influito in modo importante sul tasso di biodiversità presente nei sistemi agricoli determinandone una graduale, ma sempre più accelerata, perdita.

La Convenzione delle Nazioni Unite sulla Diversità Biologica, firmata a Rio de Janeiro il 5 giugno 1992, definisce la biodiversità (Biological Diversity) come «the variability among living organisms from all sources including, inter alia, terrestrial, marine and other aquatic ecosystems and the ecological complexes of

1. G. Barbera, Antropocene, agricoltura e paesaggio, Aboca Sansepolcro (Ar), Arezzo 2019, pp. 13-88.

2. D. Q. Fuller et al., The contribution of rice agriculture and livestock pastoralism to prehistoric methane levels: an archaeological assessment, «The Holocene», 21 (2011), pp. 743-759; B. D. Smith, M. A. Zeder, The onset of Anthropocene, «Anthropocene», 4 (2013), pp. 8-13. 
which they are part; this includes diversity within species, between species and of ecosystems» (Onu, 1992). In tale occasione vennero delineati i tre importanti obiettivi della Convenzione stessa, ovvero la conservazione della diversità biologica, l'uso sostenibile dei componenti della diversità e la giusta ed equa ripartizione dei benefici derivanti dall'utilizzo delle risorse genetiche.

È evidente che la biodiversità è stata riconosciuta come la base per i sistemi naturali di supporto alla vita sulla Terra, in quanto fornitrice di servizi ecosistemici; ma andando al di là del punto di vista ecologico, la biodiversità ha anche una valenza simbolica, culturale e sociale. Basti ricordare il ruolo decisivo che hanno i popoli indigeni nella sua conservazione e nell'identità culturale che si crea attorno a un prodotto agro-alimentare, seme, pianta, animale o trasformato che sia ${ }^{3}$.

Nel 2009 sulla rivista Nature è stato inserito, da un gruppo di esperti di scienze del sistema Terra e della sostenibilità guidato da Johan Rockström, il concetto di 'confine planetario' che ha l'ambizione di definire uno "spazio operativo sicuro" in cui poter agire senza compromettere il futuro del pianeta.

Dei 9 confini planetari identificati nell'articolo, uno fa specifico riferimento ai cambiamenti nell'integrità della biosfera (perdita di biodiversità ed estinzioni) ed è considerato, insieme a quello relativo al cambiamento climatico, un confine fondamentale da non superare per evitare che il Sistema Terra raggiunga 'un nuovo stato'4. Queste considerazioni derivano dalla consapevolezza che quasi un quarto delle specie vegetali del pianeta è a rischio di estinzione, mentre le popolazioni globali di pesci, uccelli, mammiferi, anfibi e rettili sono diminuite del $58 \%$ tra il 1970 e il 2012, riduzione che potrebbe aumentare nel corso dei prossimi anni ad un tasso sempre maggiore ${ }^{5}$.

3. M. Schultz, T.D. Tyrrell, T. Ebenhard, The 2030 Agenda and Ecosystems - A discussion paper on the links between the Aichi Biodiversity Targets and the Sustainable Development Goals, SwedBio at Stockholm Resilience Centre, Stockholm 2016.

4. L. P. Stuart, N. J. Clinton, V. L. Binbin, How to protect half of Earth to ensure it protects sufficient biodiversity, "Science Advances», 2018.

5. WWF, Risk and resilience in a new era. Living Planet Report WWF International, Gland 2016. 
Inoltre un recente studio del 2016 condotto nell'ambito della Piattaforma intergovernativa sulla biodiversità e i servizi ecosistemici (IPBES) mostra che il 40\% delle specie impollinatrici sono a rischio di estinzione, con potenziali conseguenze devastanti per il cibo e i mezzi di sussistenza, considerando che il $75 \%$ delle nostre colture alimentari dipende dagli impollinatori.

\section{Biodiversità e agrobiodiversità al centro di accordi}

internazionali, quadro di riferimento per la sostenibilità planetaria

Lagricoltura e la conseguente disponibilità di materie prime sono parametri essenziali che hanno accompagnato l'uomo nel corso della sua evoluzione, influenzando bisogni, culture e strutture sociali. Il rapporto tra uomo ed ecosistema non è quindi da considerarsi unidirezionale, bensì come un fenomeno co-evolutivo. La biodiversità è un elemento identitario di tale rapporto ed in particolare l'agrobiodiversità definita dalla FAO (Food and Agriculture Organization) nel 1999 come la varietà e variabilità di animali, piante e microrganismi che sono importanti per il cibo e l'agricoltura e che sono il risultato delle interazioni tra l'ambiente, le risorse genetiche, $\mathrm{i}$ sistemi di gestione e le pratiche usate dagli uomini.

Per decenni l'uomo, nel suo costante evolversi in termini demografici, spaziali e tecnologici, si è auto-identificato come l'unica specie in grado di dominare la natura, plasmandola secondo le sue necessità e provocando una trasformazione evidente del pianeta Terra ${ }^{6}$.

Tale processo è sì cruciale per la vita dell'essere umano ma è determinante anche per tutte le altre specie viventi che abitano l'ecosistema terrestre. Infatti, un ecosistema caratterizzato da poche singole specie appartenenti ai gruppi più resilienti, è più vulnerabile e più soggetto a rischi come la desertificazione, la salinizzazione, la colonizzazione da parte di specie allogene, l'inter ruzione di servizi eco-sistemici fondamentali e l'insicurezza alimentare, con gravi conseguenze e ripercussioni soprattutto nei contesti socio-economici fragili. È pertanto nella relazione fra natura e cultura, fra 
umano e non-umano che si cela il fondamentale ruolo della tutela della biodiversità. Nel corso degli ultimi cinquanta anni molti accordi internazionali sono stati negoziati per cercare di garantire la salvaguardia, la conservazione e la valorizzazione della biodiversità e dell'agrobiodiversità per sottolineare come essa sia fondamentale nel definire lo stato di salute di un sistema. All'inizio degli anni 2000 e più precisamente nell'aprile 2002, nell'ambito della Convenzione sulla Diversità Biologica, durante la sua Sesta conferenza delle parti (https://www.cbd.int/), e poi confermato dai capi di Stato al Summit Mondiale sullo Sviluppo Sostenibile tenutosi sempre nel 2002 a Johannesburg in Sudafrica, è stato siglato l'impegno a raggiungere entro il 2010 (Anno Internazionale della biodiversità) una riduzione significativa del tasso di perdita di biodiversità a livello globale, regionale e nazionale quale contributo alla riduzione della povertà e al raggiungimento di un modello di sviluppo sostenibile. La decima riunione della conferenza delle parti, tenutasi dal 18 al 29 ottobre 2010, a Nagoya, nella prefettura di Aichi, in Giappone, ha adottato un piano strategico rivisto e aggiornato per la biodiversità, definendo gli obiettivi di Aichi (Aichi Target) per la biodiversità, per il periodo 2011-2020. Laspetto interessante è che gli SDGs (UN Sustainable Development Goals 2030) e il piano strategico per la biodiversità si integrano e sostengono a vicenda al punto che l'attuazione di uno contribuisce al raggiungimento dell'altro.

A questo punto è possibile chiedersi: perché la biodiversità è importante per lo sviluppo sostenibile?

Come già affermato in precedenza la biodiversità è fondamentale per assicurare quei servizi ecosistemici essenziali per le aree rurali e urbane ed è possibile affermare che il benessere delle generazioni attuali e future dipende in gran parte dalla sua salvaguardia.

E l'altra domanda che dobbiamo porci è: perché lo sviluppo sostenibile è importante per la biodiversità?

Gli SDGs promuovono, in particolar modo, un cambiamento trasformativo nelle economie e nelle società ed è quindi auspicabile che porteranno a una nuova idea di governance, a livello mondiale, che integri maggiormente la salvaguardia della biodiversità con le politiche dei governi. In particolar modo l'auspicata crescita economica che contribuisce all'eradicazione della povertà deve essere per- 
seguita in modo socialmente equo e sostenibile dal punto di vista ambientale. Conseguentemente le agende dei vari governi dovranno tenere in conto un impatto ridotto sulla biodiversità e sugli ecosistemi, in modo da affrontare le molteplici dimensioni del benessere umano in modo sostenibile.

I 17 Obiettivi di Sviluppo Sostenibile (SDGs) hanno, infatti, l'obiettivo di mobilitare gli sforzi comuni per porre fine a tutte le forme di povertà, ridurre le disuguaglianze, affrontare il cambiamento climatico e promuovere la salvaguardia dell'ambiente (CBD, 2017).

Con l'adozione dell'Agenda 2030 non solo è stato espresso un chiaro giudizio sull'insostenibilità dell'attuale modello di sviluppo, ma si è superata l'idea che la sostenibilità sia unicamente una questione ambientale, a favore di una visione integrata delle diverse dimensioni dello sviluppo (economia, ambiente, società, istituzioni).

2. Simmetrie e asimmetrie fra $i$ Goals dell'Agenda 2030 e gli Aichi Target

L'Organizzazione delle Nazioni Unite per l'Alimentazione e l'Agricoltura stima che il $75 \%$ della diversità delle colture sia scomparso tra il 1900 e il $2000^{7}$.

Ad oggi, come sottolineato dai dati riportati dalla FAO stessa, 1,5 miliardi di ettari, circa il 30\% delle terre emerse è dedicato all'agricoltura, le specie vegetali utilizzabili dall'uomo per la propria alimentazione sono circa 7.000, ma solamente 150 vengono coltivate in maniera estensiva a questo scopo. Di queste, 12 forniscono quasi tre quarti dei nostri alimenti, e 4 di esse (riso, mais, grano, patata) provvedono a più della metà dell'alimentazione quotidiana mondiale. Pertanto la riduzione di agrobiodiversità, si inserisce in un più ampio fenomeno di perdita globale della biodiversità.

La biodiversità, al centro degli Aichi Targets, è un elemento trasversale, che esplicitamente o implicitamente diviene un tema rilevante per il raggiungimento di tutti gli SDGs, in

7. FAO, Commission on genetic resources for food and agriculture assessment, The state of the world's biodiversity for food and Agriculture, Rome 2019. 
particolare gli SGDs 2, 12, e 15 si occupano della sua diretta promozione e tutela (CBD, 2017).

Tabella 1 - Goal 2 (SDGs) e Aichi Targets

\begin{tabular}{|l|l|}
\hline Obiettivi di Sviluppo Sostenibile & Aichi Targets per la Biodiversità \\
\hline GOAL 2 Porre fine alla fame, & 2 - Valori integrati di biodiversità \\
raggiungere la sicurezza alimen- & 6 - Gestione sostenibile delle fonti di vita \\
tare e una migliore nutrizione, & acquatiche \\
promuovere un'agricoltura so- & 7 - Agricoltura, acquacoltura e silvicoltura \\
stenibile & sostenibili \\
& 13 - Salvaguardare la diversità genetica \\
& 18 - Conoscenza della tradizione \\
\hline
\end{tabular}

È chiaro che la biodiversità è essenziale per l'approvvigionamento alimentare ed è un fattore determinante per la qualità del cibo. Le diverse varietà di colture e razze animali utilizzate in agricoltura derivano infatti dalla diversità genetica presente nel mondo.

Più di 3 miliardi di persone - quasi la metà dell'attuale popolazione mondiale - vive in aree rurali e circa 2,5 miliardi di esse traggono il loro sostentamento dall'agricoltura, dall'allevamento, raccolta, caccia e pesca ${ }^{8}$.

Il forte aumento della popolazione mondiale esercita un'ulteriore pressione sui sistemi di produzione alimentare attraverso la rapida crescita della domanda di cibo - il quale dovrebbe aumentare del $70 \%$ entro il $2050^{9}$.

Una delle maggiori sfide del mondo è garantire l'accesso universale a cibo sufficiente, sano, economico e prodotto in modo sostenibile. Tuttavia, nonostante i grandi progressi tecnologici e la crescita economica della società, il sistema alimentare globale attuale non garantisce una adeguata e sana nutrizione per l'intera umanità, dominata da forti disuguaglianze economiche e sociali. Nel xxi secolo, nonostante possa apparire strano, la malnutrizione risulta come seconda causa di morte al mondo e, assieme al cambiamento climatico, si profila come una delle più importanti sfide globali. Attualmente, a livello mondiale circa 795 milioni di persone sono considerate sottonutrite, due miliardi considerate sovrappeso o obese, mentre due miliardi mancano

8. FAO, Statistical Yearbook, World Food and Agriculture, Rome 2013.

9. M. Schultz, T.D. Tyrrell, T. Ebenhard, op. cit. 
delle vitamine e dei minerali essenziali necessari per una nutrizione adeguata. I fattori di rischio dietetici sono oggi la prima causa di morbilità e mortalità, tra questi spicca la scarsa qualità della dieta associata alla scarsa assunzione di frutta e verdura e di altri alimenti vegetali e animali ricchi di sostanze nutritive. Questi elementi, che ricoprono perciò vitale importanza possono essere in gran parte forniti dalla biodiversità alimentare. Nonostante ciò, le diete alimentari stanno diventando sempre più uniformi e semplificate, povere di micronutrienti, e il settore agro-alimentare attuale si concentra sull'aumento della produzione di un numero ristretto di colture di base ${ }^{10}$. A livello globale, l'incidenza della fame nel mondo è scesa dal 15\% del 2000-2002 all' $11 \%$ del 2014-2016. Tuttavia, se la tendenza attuale dovesse confermarsi, sarà in ogni caso molto difficile raggiungere il goal 2 entro il 2030. Il punto di congiunzione tra il Goal 2 e gli Aichi Targets sta proprio nella considerazione che tutti gli approcci all'agricoltura a basso input e basati sulla ricostruzione di agroecosistemi sono particolarmente adatti per sostenere la conservazione e l'uso sostenibile della biodiversità. Infatti la diversità genetica in agricoltura è un elemento chiave della sicurezza alimentare in quanto contribuisce a garantire l'evoluzione delle specie in grado di adattarsi alle mutevoli condizioni ambientali, nonché alla resistenza a particolari malattie e parassiti.

Inoltre le conoscenze e le pratiche tradizionali ereditate da generazioni dalle comunità indigene e locali possono spesso fornire misure preziose e comprovate di conservazione e uso sostenibile delle specie vegetali e delle razze animali.

Infine è possibile sottolineare che una governance più attenta e responsabile nel complesso migliorerebbe anche la componente reddituale dei piccoli agricoltori e delle comunità locali (ASVIS, 2020).

La ricerca sviluppata negli ultimi anni ${ }^{11}$ dimostra infatti che il grado di diversificazione all'interno dei sistemi di coltivazione può avere effetti importanti sulla produttività

10. D. Hunter et al., Agrobiodiversity for healthy, diverse diets and food systems, «CGIAR», 2016.

11. A. J. Bennett, et al., Meeting the demand for crop production: the challenge of yield decline in crops grown in short rotations, «Biological Reviews», 87 (2012), pp. 52-71. 
delle colture stesse. Infatti, i rendimenti possono essere ridotti tra il 3 e il $57 \%$ per le colture principali coltivate in sequenze a rotazione breve e monocolture rispetto a quelle in sequenze a rotazione estesa che includono più specie di colture. Minore produttività in cicli di rotazione meno diversificata è attribuibile a numerosi fattori interattivi, tra cui l'aumento e maggiori danni causati da insetti nocivi ed erbacce, la compattazione del suolo, l'esaurimento e la perdita di nutrienti, la ridotta fertilità e la ridotta disponibilità di acqua nel suolo.

La produzione sostenibile di cibo per una popolazione mondiale in crescita richiede quindi diverse sfide da affrontare, e deve essere affiancata a modelli di produzione e consumo sostenibili, per evitare che gli sforzi fatti alle base della filiera non vadano vanificati ${ }^{12}$. A tal riguardo, il concetto di "modelli di produzione e consumo sostenibili" non è nuovo nel dibattito internazionale. Già da diversi decenni si è affermata l'importanza di agire dal lato della produzione, riducendo al minimo l'uso delle risorse naturali e attraverso un uso efficace e dal lato del consumo, sensibilizzando i cittadini a forme di consumo responsabili. Tale approccio è inserito nel Goal 12.

Tabella 2 - Goal 12 (SDGs) e Aichi Targets

\begin{tabular}{|l|l|}
\hline Obiettivi di Sviluppo Sostenibile & Aichi Targets per la Biodiversità \\
\hline $\begin{array}{l}\text { GOAL 12 - Garantire modelli so- } \\
\text { stenibili di produzione e di con- } \\
\text { sumo }\end{array}$ & $\begin{array}{l}\text { biodiversità } \\
7 \text { - Agricoltura, acquacoltura e silvicoltura } \\
\text { sostenibili } \\
4 \text { - Produzione e consumo sostenibili } \\
\text { 8 - Riduzione dell'inquinamento } \\
\text { 6- Gestione sostenibile delle fonti di vita } \\
\text { acquatiche } \\
19 \text { - Condivisione di informazioni e co- } \\
\text { noscenze }\end{array}$ \\
\hline
\end{tabular}

Il consumo e la produzione di tutti i beni e servizi richiedono la trasformazione di molte risorse naturali, che a loro volta incidono sulla biodiversità. Gli attuali modelli di consumo e produzione non sostenibili possono minare la 
capacità degli ecosistemi di fornire servizi alle industrie e alle comunità che si basano su di essi.

Gli ecosistemi sani sono la base per un consumo e una produzione sostenibile. A sua volta, il consumo e la produzione sostenibili sono necessari per ridurre al minimo gli effetti negativi sulla biodiversità e garantire la disponibilità a lungo termine dei benefici che gli ecosistemi sani offrono. Pertanto, non riconoscere i legami tra ecosistemi, consumo e produzione, genera gravi problemi all'intero sistema. Al contrario, se i legami vengono riconosciuti generando equilibrio agli agroecosistemi, la biodiversità può rivelarsi un valore fondamentale per uno sviluppo economico e sociale continuo, equo e resiliente a shock come il cambiamento climatico e le fluttuazioni dei mercati finanziari ${ }^{13}$.

Il numero di disastri estremi legati al clima, tra cui l'innalzamento delle temperature, siccità, inondazioni e tempeste, è raddoppiato dall'inizio degli anni '90. Gli impatti diretti, come la riduzione, il riadattamento e la riconversione degli habitat, possono portare alla perdita e all'estinzione dell'agrobiodiversità e hanno forti impatti sui sistemi alimentari ${ }^{14}$. Essi danneggiano la produttività agricola, contribuendo a ridurre la disponibilità di cibo, con effetti a catena che causano l'aumento dei prezzi di prodotti agroalimentari e perdite di reddito, che riducono l'accesso delle persone al cibo, limitando la domanda.

In molte aree, eventi climatici estremi sono aumentati in numero ed intensità, in particolare le temperature medie si stanno spostando verso l'alto. Il calore estremo è associato a un aumento della mortalità, a una minore capacità di lavoro, a rendimenti agricoli più bassi e ad altre conseguenze che minano la sicurezza alimentare e la nutrizione. La fame è significativamente peggiore nei paesi con sistemi agricoli altamente sensibili alle precipitazioni, alla variabilità della temperatura e alla grave siccità; dove il sostentamento di un'alta percentuale della popolazione dipende dall'agricoltura e dove il paese non dispone di misure di sostegno sufficienti per contrastare il fenomeno. In sintesi, per quasi il $36 \%$ dei paesi che hanno registrato un aumento della denu-

13. M. Schultz, T.D. Tyrrell, T. Ebenhard, op. cit.

14. K. S. Zimmerer, The biodiversity of food and agriculture (Agrobiodiversity) in the anthropocene: Research advances and conceptual framework, "Anthropocene», 25 (2019). 
trizione dal 2005, ciò è coinciso con il verificarsi di una grave siccità agricola in questi luoghi. La ricerca sulla perdita dell'agrobiodiversità causata da tali cambiamenti globali si è finora concentrata sulla capacità di adattamento delle specie e delle varietà vegetali, molto meno si sa sui processi sociali e politico-ecologici di vulnerabilità, resilienza e trasformazione dei territori ${ }^{15}$. Inoltre il cambiamento dei modelli di consumo richiederà un coinvolgimento attivo del pubblico poiché un numero crescente di paesi e di popolazione adotta i modelli di consumo delle società economicamente avanzate. La sensibilizzazione e l'accesso alle informazioni su varie dimensioni dello sviluppo sostenibile, compresi la biodiversità e l'ecosistema, sono i prerequisiti per spostare le scelte di consumo e gli stili di vita.

Sempre in riferimento all'Agenda 2030, l'obiettivo che più in specifico si preoccupa del servizio e sull'uso sostenibile degli ecosistemi e delle specie del pianeta, è il Goal 15.

Tabella 3 - Goal 15 (SDGs) e Aichi Targets
Goal 15 -proteggere, ripristinare e favorire un uso sostenibile dell'ecosistema terrestre, gestire sostenibilmente le foreste, contrastare la desertificazione, arrestare e far retrocedere il degrado del terreno, e fermare la perdita di diversità biologica
2 - Valori integrati di biodiversità

4 - Produzione e consumo sostenibili

5 - Perdita dimezzata o ridotta di habitat

7 - Agricoltura, acquacoltura e silvicoltura sostenibili

9 - Specie aliene invasive prevenute e controllate

11 - Aree protette

12 - Riduzione del rischio di estinzione delle risorse

14 - Servizi ecosistemici

15 - Restauro e resilienza dell'ecosistema 16 - Accesso ai benefici genetici e condivisione dei benefici

La conservazione, il ripristino e l'uso sostenibile degli ecosistemi terrestri e delle acque dolci interne sono essenziali per lo sviluppo sostenibile. In tale contesto è bene ricordare ecosistemi a volte dimenticati come le foreste e i sistemi montani. Le foreste coprono circa il $30 \%$ dell'area terrestre, contengono l' $80 \%$ della biomassa terrestre e forniscono l'habitat 
per oltre la metà delle specie animali e vegetali conosciute al mondo. Anche se il tasso di perdita delle foreste annuale sta rallentando negli ultimi decenni, è ancora motivo di forte preoccupazione a causa della perdita costanti in aree con un valore ecologico particolarmente elevato. I dati dimostrano come tra il 1990 e il 2015, le aree occupate dalle foreste siano diminuite dal 31,7\% sul totale delle terre emerse al 30,7\%. Questa perdita è riconducibile alla conversione delle foreste in terre per altri tipi di uso, come l'agricoltura, o alla costruzione di infrastrutture. Allo stesso tempo, però, molte terre sono state trasformate in foreste grazie alla piantumazione di alberi e piante. Se si mettono i due processi a confronto, si può stimare che la perdita annuale globale di aree coperte da foreste è passata dai 7,3 milioni di ettari del 1990 ai 3,3 milioni di ettari all'anno tra il 2010 e il 2015 (ASVIS, 2020). A livello globale è interessante sottolineare la presenza di programmi legati alla realizzazione di aree protette che potrebbero arricchire in modo sostanziale il potenziale della biodiversità (Aichi target 11).

La ricerca delle simmetrie e delle asimmetrie tra i due strumenti è utile per comprendere come l'Agenda 2030 possa contribuire all'attuazione dell'attuale Piano Strategico per la Biodiversità 2011-2020 (e viceversa) in quanto è necessario considerare un possibile processo per lo sviluppo di un quadro post-2020 ${ }^{16}$.

Tuttavia è possibile sottolineare come spesso quanto messo in rilievo dagli Aichi Targets, non si rifletta adeguatamente negli Obiettivi di Sviluppo Sostenibile (SDGs). Infatti molto spesso non sono così chiari i collegamenti che si possano instaurare tra indicatori. Probabilmente sarebbe necessario che gli indicatori andassero oltre le misure convenzionali della produzione agricola e della resa produttiva, per misurare meglio ad esempio la qualità nutrizionale, la diversità nutrizionale dei sistemi alimentari e la diversità dietetica. È dunque necessario un maggiore sforzo di integrazione sul tema biodiversità in modo che i molteplici beni e servizi ecosistemici che essa può fornire per la nutrizione e il benessere umano siano meglio tracciati ed evidenziati ${ }^{17}$.

16. M. Schultz, T.D. Tyrrell, T. Ebenhard, op. cit.

17. D. Hunter et al., Agrobiodiversity cit. 
Lintegrazione dei valori della biodiversità nei processi di pianificazione e nei sistemi nazionali di contabilità e rendicontazione richiederà alla governance di valutare adeguatamente i vari aspetti. Gli sforzi per migliorare la valutazione della biodiversità dovrebbero includere strumenti e metodi che riconoscano i valori sociali e culturali, oltre ai valori economici, e dovrebbero essere condotti in modi che incoraggino l'uso sostenibile della biodiversità a tutti i livelli. Per raggiungere questo obiettivo sarà necessaria una combinazione di politiche nazionali e locali.

\section{Conclusioni}

L'agrobiodiversità è un elemento cardine per l'attuazione di specifiche soluzioni gestionali e politiche necessarie per lo sviluppo sostenibile, la sicurezza alimentare e nutrizionale, la conservazione della biodiversità, l'equità sociale e la giustizia. Lanalisi dell'agrobiodiversità dimostra quindi una particolare promessa nell'aiutare a comprendere e a rispondere alle intensificate interazioni umane con i sistemi terrestri e all'accelerazione dei cambiamenti globali ${ }^{18}$.

Al termine "dieta sana" non corrisponde una definizione unica, tuttavia tra le componenti generali di una dieta sana secondo la definizione dell'Organizzazione Mondiale della Sanità (OMS) vi è una varietà di prodotti agroalimentari esito della diversità alimentare. Seppure gran parte di essa sia stata trascurata o persa ha un enorme potenziale per fornire la ricchezza naturale di nutrienti di cui l'essere umano ha bisogno. Pertanto la promozione della diversificazione dei sistemi di produzione e del consumo di cibo è e sarà necessaria per ottenere risultati nutrizionali ottimali, ma non solo, è e sarà anche importante per la resilienza e la sostenibilità dei sistemi agro-alimentari attuali e futuri.

Molte agenzie, tra cui FAI, la Convenzione sulla diversità biologica (CBD), l'OMS e Bioversity International riconoscono l'importante ruolo della biodiversità alimentare in questo slancio per rimodellare i sistemi alimentari.

I paesaggi sono diventati sistemi socio-ecologici complessi in cui le attività antropogeniche e i fattori biofisici interagiscono su più scale. L'integrazione dei processi di 
sviluppo socio-economico nelle strategie di conservazione come mezzo di gestione sostenibile delle risorse richiede una profonda comprensione delle interazioni tra attività umane e processi naturali. Recenti studi confermano la necessità di promuovere sinergie tra la conservazione della biodiversità, la sua gestione e l'attuazione di politiche volte a migliorare i mezzi di sussistenza umani ${ }^{19}$.

Sfruttare le opportunità per promuovere meglio la biodiversità alimentare come strategia chiave per diete sane e sistemi alimentari sostenibili è necessario per il sistema globale attuale il quale si trova di fronte all'impellente sfida del cambiamento climatico ${ }^{20}$. Ecco dunque che c'è un urgente bisogno di un approccio integrato, che affronti gli ostacoli di natura tecnica, economica o sociale, che mantenga i sistemi di supporto vitale della Terra garantendo al contempo un continuo progresso sociale.

\section{Bibliografia}

Barbera G. 2019. Antropocene, agricoltura e paesaggio, Aboca Sansepolcro (Ar), Arezzo.

Bennett A.J. et al. 2012. Meeting the demand for crop production: the challenge of yield decline in crops grown in short rotations, "Biological Reviews», 87, pp. 52-71.

Bridgewater P., Régnier M., Cruz García R. 2016. Implementing Sdg 15: Can large-scale public programs help deliver biodiversity conservation, restoration and management, while assisting human development?, «Natural Resources Forum», 39, pp. 214-223.

Fao. 2019. Commission on genetic resources for food and agriculture assessment, The state of the world's biodiversity for food and Agriculture, Rome, Italy.

- 2013. Statistical Yearbook, World Food and Agriculture, Rome, Italy.

Fuller D.Q. et al. 2011. The contribution of rice agriculture and livestock pastoralism to prehistoric methane levels: an archaeological assessment, «The Holocene», 21, pp. 743-759.

Harari Y.N. 2015. Sapiens. A brief history of humankind, Random UK, London.

19. P. Bridgewater, M. Régnier, R. Cruz García, Implementing Sdg 15: Can large-scale public programs help deliver biodiversity conservation, restoration and management, while assisting human development?, «Natural Resources Forum», 39 (2016), pp. 214-223.

20. D. Hunter et al., op. cit. 
Hunter D. et al. 2016. Agrobiodiversity for healthy, diverse diets and food systems, «CGIAR».

IPBES. 2016. Summary for policymakers of the assessment report of the intergovernmental science-policy platform on biodiversity and ecosystem services on pollinators, pollination and food production, Bonn, Germany.

Lim, M. M. L., P. Søgaard Jørgensen, C. A. Wyborn. 2018. Reframing the sustainable development goals to achieve sustainable development in the Anthropocene - a systems approach, «Ecology and Society», 23, pp. 22 sgg.

Opoku A. 2018. Biodiversity and the built environment: Implications for the Sustainable Development Goals (Sdgs), «Resources Conservation and Recycling», 141, pp.1-7.

Rockstrom, J. et al. 2009. A safe operating space for humanity, «Nature» 461, pp. 472-475.

Salleh A. 2016. Climate, Water, and Livelihood Skills: A Post-Development Reading of the Sdgs, «Globalizations», 13, pp. 952-959.

Schultz M., Tyrrell T.D., Ebenhard, T. 2016. The 2030 Agenda and Ecosystems - A discussion paper on the links between the Aichi Biodiversity Targets and the Sustainable Development Goals, SwedBio at Stockholm Resilience Centre, Stockholm, Sweden.

Smith B. D., Zeder M. A. 2013. The onset of Anthropocene, «Anthropocene», 4, pp. 8-13.

Stuart L. P., Clinton N. J. Binbin V. L. 2018. How to protect half of Earth to ensure it protects sufficient biodiversity, «Science Advances».

United Nations. 1992. Conventions on Biological Diversity. https:// www.cbd.int/convention/text/

WWF. 2016. Risk and resilience in a new era. Living Planet Report WWF International, Gland, Switzerland.

Zimmerer K. S. 2019. The biodiversity of food and agriculture (Agrobiodiversity) in the anthropocene: Research advances and conceptual framework, «Anthropocene», 25. 
Maria Pia Genesin

Il 2015, come sottolineato dalla Commissione Ue nella sua Comunicazione sul futuro sostenibile dell'Europa ${ }^{1}$, ha rappresentato un anno importante per la presa di coscienza a livello mondiale della necessità di fissare obiettivi di sviluppo sostenibile per il prossimo futuro.

Nel mese di luglio è stato approvato dall'Assemblea generale delle Nazioni Unite il programma di azione Addis Abeba, che delinea la cornice finanziaria per il conseguimento degli obiettivi dello sviluppo sostenibile ${ }^{2}$. A settembre è stata adottata la c.d. Agenda 2030 (Trasformare il nostro mondo: l'Agenda 2030 per lo sviluppo sostenibile), approvata all'unanimità dai 193 Paesi membri delle Nazioni Unite ${ }^{3}$. Nel dicembre è stato raggiunto l'Accordo di

1. Comunicazione della Commissione, Il futuro sostenibile dell'Europa: prossime tappe. Lazione europea a favore della sostenibilità, 22 novembre 2016, Com(2016) 739 final.

2. Risoluzione delle Nazioni Unite $\mathrm{A} / \mathrm{Res} / 69 / 313$.

3. Risoluzione delle Nazioni Unite A/Res/70/1. In argomento si veda M. Montini, F. Volpe, Sustainable development goals: molto rumore per nulla?, «Rivista giuridica dell'ambiente», 2015, n. 3, pp. 489 sgg. 
Parigi, in occasione della Conferenza sui cambiamenti climatici-Cop 21, al centro del quale vi è l'impegno delle Parti contraenti nel senso di una significativa riduzione di emissioni di CO2 nell'atmosfera ${ }^{4}$.

Si ricorda, infine, che il 2015 è anche l'anno della Lettera enciclica Laudato si' di Papa Francesco sulla cura della casa comune, in cui si afferma a chiare lettere che «l'ambiente è un bene collettivo, patrimonio di tutta l'umanità e responsabilità di tutti $\star^{5}$.

L'Agenda 2030 pone 17 obiettivi (Sustainable development goals) e 169 target che, nel loro insieme, ampliano gli 8 obiettivi di sviluppo del millennio (Millennium development goals) concordati nel 2000 a livello di Nazioni Unite e giunti a scadenza nel 2015. L'Agenda 2030 si fonda su cinque P - People, Planet, Prosperity, Peace, Partnership - e si pone in linea di continuità con i principi della Dichiarazione delle Nazioni Unite sull'ambiente umano, adottata a Stoccolma nel 1972, in cui si afferma che «difendere e migliorare l'ambiente per le generazioni presenti e future è diventato per l'umanità un obiettivo imperativo», e con i principi della Dichiarazione su ambiente e sviluppo adottata a Rio de Janeiro nel 1992 in occasione della Conferenza su ambiente e sviluppo - Earth summit, da cui è originata l'Agenda 21. Grazie a tale conferenza il concetto di sviluppo sostenibile - ripreso dal Rapporto delle Nazioni Unite Our common future del 1987 (c.d. rapporto Brundtland) - è entrato a far parte del lessico istituzionale e della società civile. Nel 2012 si è svolta la Conferenza delle Nazioni Unite sullo sviluppo sostenibile, detta anche Rio + 20; in tale occasione è stato particolarmente valorizzato il ruolo dell'imprenditoria privata e dei partenariati pubblico-privati nel perseguimento dell'obiettivo di uno sviluppo economico compatibile con la salvaguardia delle risorse a disposizione delle generazioni future ${ }^{6}$.

4. In proposito si veda L. Aristei, L'Accordo di Parigi: obiettivi e disciplina, «Rivista Quadrimestrale di Diritto dell'Ambiente», 2017, n. 3, pp. 73 sgg.

5. Per un approfondimento sui contenuti dell'enciclica Laudato si' di Papa Francesco si veda L. Boi, Pensare la relazione complessa dell'uomo con la natura, «Agricoltura-IstituzioniMercati», 2015, nn. 2-3, pp. 13 sgg.; N. Ferrucci, Il diritto e il rovescio: riflessioni del giurista sull'ambiente alla luce dell'enciclica Laudato si', ivi, 2015, nn. 2-3, pp. 74 sgg.

6. Per una ricognizione a tutto tondo del valore giuridico di tali documenti e del loro 
Sostenibilità ambientale, sicurezza alimentare e politiche
L'Agenda 2030 riconosce la responsabilità primaria degli Stati nella gestione della propria economia e nel perseguimento degli obiettivi di sviluppo sostenibile e prevede azioni di follow-up per misurare e monitorare i risultati raggiunti. Lo sviluppo sostenibile deve, però, essere obiettivo che impegna non solo l'azione delle istituzioni - Legislatore e pubbliche amministrazioni - ma anche la società civile e il mondo imprenditoriale. Con riguardo specifico al comparto produttivo alimentare occorre sottolineare il ruolo importante del settore agricolo poiché la maggior parte degli alimenti in commercio ha origine nella produzione primaria. Centrale diviene il passaggio dal modello di agricoltura monofunzionale e di allevamento intensivo, che si è consolidato a partire dal secondo dopo guerra con l'obiettivo di garantire l'autonomia alimentare, ad un diverso modello che sappia coniugare l'esigenza di produrre un reddito soddisfacente con il rispetto dell'ambiente, degli animali, delle risorse naturali $^{7}$. Le imprese impegnate nel settore della trasformazione, della distribuzione, della ristorazione - dalle microimprese alle multinazionali - devono impegnarsi in una crescita economica che sfrutti l'innovazione e la creatività per realizzare uno sviluppo sostenibile che sia tale nelle sue tre dimensioni - economica, sociale, ambientale. Le imprese devono contribuire a cambiare i modelli di consumo. I consumatori, a loro volta, sono chiamati a sviluppare e coltivare una maggiore sensibilità verso comportamenti di consumo responsabile, il che, sotto il profilo alimentare, significa essenzialmente ridurre, sino all'obiettivo ottimale di evitare, lo spreco di cibo.

contenuto si veda A. Crosetti et al., Introduzione al diritto dell'ambiente, Laterza, Roma-Bari 2018.

7. Sull'evoluzione del ruolo dell'agricoltura, nel senso di una sua acquisita multidimensionalità, inclusiva della tutela ambientale, si veda G. Buia, Agricoltura multifunzionale e produzione integrata: profili giuridici, «Rivista Quadrimestrale di Diritto dell'Ambiente», 2019, n. 1, pp. 42 sgg. e ivi per ulteriori riferimenti bibliografici sull'argomento. Sul tema si veda, altresì, M. Monteduro, Ius et rus: la rilevanza dell'agroecologia per il diritto, «Rivista Quadrimestrale di Diritto dell'Ambiente», 2019, n. 2, pp. 4 sgg.; M. Troisi, $A n$ notazioni sulla tutela costituzionale dell'agroecologia: il problematico inquadramento nell'attuale ripartizione delle competenze, ivi, 2016, n. 2, pp. $101 \mathrm{sgg}$. Per uno sguardo oltralpe su questi temi si veda L. Colella, La «loi agriculture ed alimentation» nel modello francese: il primo passo per l'affermazione del «diritto al cibo sano e sostenibile» come valore costituzionale, "Diritto e giurisprudenza agraria, alimentare e dell'ambiente», 2018, n. 6, p. 1 sgg. Più in generale sul tema G. Sgarbanti, P. Borghi, A. Germanò (a cura di), Il divenire del diritto agrario italiano ed europeo tra sviluppi tecnologici e sostenibilità, Giuffrè, Milano 2014. 
Il percorso verso l'obiettivo di coniugare produttività, consumo, sostenibilità è lungo e non facile. L'Agenda 2030 pone goals importanti, che dovrebbero segnare un cambiamento epocale, di cui, però, si vedono ancora solo pochi, concreti segnali. Si nota interesse verso l'adozione di modelli di produzione e di consumo sostenibili, ma la velocità con cui si investe in tale direzione non è, al momento, in grado di competere con la velocità con cui crescono i rischi ambientali legati all'inquinamento e ai cambiamenti climatici.

Il Rapporto Asvis 2018 segnala che non si è ancora concretizzata «la discontinuità culturale e di scelte strategiche» necessaria per raggiungere, entro il 2030, i 17 obiettivi dell'Agenda 2030, alcuni dei quali impongono risultati già entro il 2020. Lo scenario pare essere ancora quello di «business as usual», in cui non si avvertono cambiamenti significativi nelle attitudini e nelle priorità delle persone e non ci sono evoluzioni significative nella tecnologia, nell'economia, nelle politiche di contrasto alle emissioni di CO2. Occorre, invece, sviluppare un modello culturale di business integrato, improntato non alla massimizzazione del profitto, ma alla realizzazione di un equo contemperamento fra le esigenze dell'economia, quelle della società, quelle dell'ambiente.

Leggermente più ottimista appare essere il Rapporto Asvis 2019 che segnala un maggiore sostegno da parte della società civile per azioni coraggiose, anche se costose nel breve termine; si può affermare che si sia sviluppata una presa di coscienza globale che non vi sia più tempo.

\section{Gli alimenti nell'Agenda 2030}

Gli alimenti sono ampiamente interessati dagli obiettivi dell'Agenda 2030.

L'obiettivo n. 2 intende garantire la sicurezza alimentare nel senso della disponibilità di cibo sufficiente; entro il 2030 deve essere raddoppiata la produttività agricola e il reddito dei produttori di cibo su piccola scala ${ }^{8}$. Entro il 2030 biso-

8. Il tema connesso all'obiettivo n. 2 è dunque quello della sovranità alimentare nel quale trova espressione il diritto al cibo delle popolazioni più povere. Sul tema della sovranità alimentare, inteso come diritto dei piccoli produttori agricoli di partecipare al processo produttivo del cibo, governato dalle logiche di regolazione del commercio agroalimentare internazionale, si veda, per tutti, G. Zagrebelsky, Un concetto costituzionale: 
Sostenibilità ambientale, sicurezza alimentare e politiche gna garantire sistemi di produzione alimentare sostenibili e implementare pratiche agricole resilienti che aumentino la produttività e la produzione, che aiutino a proteggere gli ecosistemi, che rafforzino la capacità di adattamento ai cambiamenti climatici, a condizioni meteorologiche estreme e che migliorino progressivamente la qualità del suolo ${ }^{9}$.

Con riguardo alla sicurezza alimentare una distinzione consueta negli studi giuridici riguarda la contrapposizione fra food security e food safety ${ }^{10}$. La prima accezione attiene al profilo qualitativo/quantitativo, da intendersi come garanzia per tutte le persone di poter avere, in ogni momento, accesso fisico, sociale ed economico ad alimenti sufficienti, sicuri e nutrienti che garantiscano i loro bisogni e preferenze alimentari per condurre una vita attiva e sana, secondo la definizione di food security proposta dalla Fao in occasione del World Food Summit di Roma del 1996; la seconda accezione è più restrittiva, è specifica del diritto alimentare ed attiene al profilo qualitativo, da intendersi come disponibilità di cibo sicuro dal punto di vista igienico-sanitario ${ }^{11}$.

L'obiettivo di garantire cibo sicuro, nell'accezione di sicurezza alimentare integrata proposta dallo Fao, a livello globale è lungi dall'essere raggiunto. Cresce sempre più il divario fra la popolazione mondiale malnutrita e quella ipernutrita; cresce sempre più il consumo di risorse am-

sovranità alimentare, «Diritto agroalimentare», 2017, n. 3, pp. 435 sgg.; A. Rinella, Food sovereignty, «Rivista Quadrimestrale di Diritto dell'Ambiente», 2015, n. 1, pp. 15 sgg.; L. Paoloni, I nuovi percorsi della food security: dal «diritto al cibo adeguato» alla "sovranità alimentare», «Diritto e giurisprudenza agraria, alimentare e dell'ambiente», 2011, n. 3, pp. 159 sgg.

9. Si tratta, dunque, di conciliare il diritto al cibo con la tutela dell'ambiente, sviluppando sistemi di produzione agricola sostenibili, nel rispetto della biodiversità. Sul punto si veda M. Pierri, Diritto al cibo, diversità alimentare e agrobiodiversità: quali strumenti di tutela? Osservazioni su alcune esperienze significative tra diritto internazionale e sovranità alimentare, «Rivista Quadrimestrale di Diritto dell'Ambiente», 2016, n. 2, pp. 33 sgg. In argomento si vedano anche M. Monteduro, Diritto dell'ambiente e diversità alimentare, ivi, 2015, n. 1, pp. 88 sgg.; F. Mignella Calvosa, F. Pilozzi, S. Totaforti, Disarticolazione del rapporto terrauomo-cibo e politiche dell'alimentazione, ivi, 2015, n. 1, pp. 41 sgg.; G. Rossi, Diritto dell'ambiente e diritto dell'alimentazione, ivi, 2015, n. 1, pp. 3 sgg.

10. L. Costato et al., Compendio di diritto alimentare, Cedam, Milano 2019, p. 2.

11. Per approfondimenti si rinvia a L. Giacomelli, Diritto al cibo e solidarietà. Politiche e pratiche di recupero delle eccedenze alimentari, «Osservatorio costituzionale», 2018, n. 1, pp. 43 sgg.; M. Giuffrida, Il diritto fondamentale alla sicurezza alimentare tra esigenze di tutela della salute umana e promozione della libera circolazione delle merci, «Rivista di diritto alimentare», 2015, n. 3, pp. 34 sgg. 
bientali limitate - acqua, suolo - per produrre cibo industriale a basso costo e di scarsa qualità nutrizionale, che contribuisce all'aumento del fenomeno dell'obesità nelle fasce più povere della popolazione dei paesi industrialmente avanzati. La Fao stima che se la popolazione mondiale dovesse raggiungere il tetto dei 9,6 miliardi entro il 2050, sarebbe necessario, per la sola produzione alimentare, l'equivalente di tre pianeti.

Lobiettivo n. 12 intende garantire modelli sostenibili di produzione e di consumo; entro il 2030 bisogna dimezzare lo spreco alimentare globale pro capite a livello di vendita al dettaglio e dei consumatori e ridurre le perdite di cibo durante le catene di produzione e di fornitura, comprese le perdite post-raccolto. Entro il 2030 occorre accertarsi che tutte le persone, in ogni parte del mondo, abbiano le informazioni rilevanti e la giusta consapevolezza dello sviluppo sostenibile e di uno stile di vita in armonia con la natura.

Lobiettivo n. 15 riguarda l'uso sostenibile dell'ecosistema terrestre.

A livello delle Nazioni Unite, il monitoraggio e la revisione dei progressi compiuti verso il conseguimento degli obiettivi di sviluppo sostenibile si svolgono ogni anno nel mese di luglio presso il Forum politico di alto livello, e ogni quattro anni a livello di capi di Stato e di governo.

\section{Obiettivi di sviluppo sostenibile e ruolo dell'Unione europea}

L'Ue riveste un ruolo centrale nell'attuazione degli obiettivi di sviluppo sostenibile dell'Agenda 2030. L'Ue ha svolto un ruolo determinante nella definizione dell'agenda globale 2030 , che si pone in linea con la visione europea e rappresenta, a livello mondiale, un modello per lo sviluppo sostenibile. L'Ue rivendica espressamente il proprio ruolo di leader in tale senso, ribadendolo anche nei suoi documenti più recenti ${ }^{12}$; tale vocazione è particolarmente evidente con

12. Si veda in proposito la Comunicazione della Commissione, Il Green deal europeo, 11 dicembre 2019, Com(2019) 640 final, che si pone l'ambizioso obiettivo di attribuire al continente europeo il primato per l'impatto climatico zero entro il 2050, attraverso una strategia di crescita sostenibile ed inclusiva. Costituisce una declinazione di questo progetto la strategia dal produttore al consumatore (from farm to fork) che intende incentivare sistemi alimentari sostenibili che permettano di instaurare un circuito virtuoso persone sane-società sane-pianeta sano. La strategia in questione è imprescindibile per conseguire gli obiettivi dell'Agenda Onu 2030. In proposito si rinvia alla Comunicazio- 
Sostenibilità ambientale, sicurezza alimentare e politiche riguardo al settore alimentare ed emerge da una serie di documenti di soft law, come tali non vincolanti ma dal forte valore programmatico, prodotti dalle istituzioni eurounitarie.

Si ricorda, a tal proposito, la Comunicazione della Commissione «Il futuro sostenibile dell'Europa: prossime tappe. Lazione europea a favore della sostenibilità» del novembre 2016, in cui si sottolinea la piena integrazione degli obiettivi di sviluppo sostenibile nel quadro strategico europeo e nelle priorità della Commissione ${ }^{13}$. In senso analogo si è espresso anche il Documento della Commissione di riflessione verso un'Europa sostenibile entro il $2030^{14}$, in cui si afferma il ruolo dell'Ue quale «pioniere mondiale dello sviluppo sostenibile» e si sottolinea la necessità della transizione dall'economia lineare, improntata alla logica del consumo delle risorse, all'economia circolare improntata alla logica del riutilizzo, della riparazione, del riciclaggio ${ }^{15}$. Queste politiche sono integrate da iniziative nel campo della ricerca e dell'innovazione in materia di sicurezza alimentare e nutrizionale, quale Food 2030. Food 2030 è una piattaforma per il dialogo, con l'obiettivo di creare un quadro politico coerente in materia di ricerca e innovazione per la sicurezza alimentare e nutrizionale. Già nel 2010 era stata emanata la Comunicazione della Commissione, Europa 2020. Una strategia per una crescita intelligente, sostenibile e inclusiva ${ }^{16}$.

Europa 2020 presenta tre priorità che si rafforzano a vicenda:

ne della Commissione, Una strategia «Dal produttore al consumatore» per un sistema alimentare equo, sano e rispettoso dell'ambiente, 20 maggio 2020, Com(2020) 381 final. Costituisce espressione del Green deal europeo anche il nuovo piano di azione per l'economia circolare che raccomanda all'Unione europea di accelerare la transizione verso un modello di crescita rigenerativo, elemento indispensabile di un processo evolutivo verso un'economia climaticamente neutra. In proposito si veda la Comunicazione della Commissione, Un nuovo piano di azione per l'economia circolare. Per un'Europa più pulita e più competitiva, 11 marzo 2020, Com(2020) 98 final. Per un commento critico si veda A. Muratori, Il nuovo piano d'azione per l'economia circolare: buoni propositi, ma anche un bel po' di aria fritta, «Ambiente e sviluppo», 2020, n. 4, pp. 289 sgg.

13. Comunicazione della Commissione, 22 novembre 2016, Com(2016) 739 final.

14. Commissione europea, 30 gennaio 2019, Com(2019) 22.

15. In proposito si veda anche la Comunicazione della Commissione, L'anello mancante - Piano d'azione dell'Unione europea per l'economia circolare, 2 dicembre 2015, Com(2015) 614 final.

16. Comunicazione della Commissione del 3 marzo 2010, Com(2010) 2020 def. 
- crescita intelligente: sviluppare un'economia basata sulla conoscenza e sull'innovazione;

- crescita sostenibile: promuovere un'economia più efficiente sotto il profilo delle risorse, più verde e più competitiva;

- crescita inclusiva: promuovere un'economia con un alto tasso di occupazione che favorisca la coesione sociale e territoriale

Lo sviluppo sostenibile è un obiettivo fondamentale dell'Unione, come previsto dall'articolo 3, paragrafo 3, del trattato sull'Unione europea (Tue) ${ }^{17}$; lo sviluppo sostenibile, come esplicato a livello di fonti primarie dell'Unione europea, implica una crescita economica equilibrata e un'economia sociale di mercato che, pur restando fortemente competitiva, mira alla piena occupazione e al progresso sociale e si fonda su un elevato livello di tutela e di miglioramento della qualità dell'ambiente, promuovendo il progresso scientifico e tecnologico.

Spesso si afferma che, nel contesto europeo, il problema della insicurezza alimentare, legato alla mancanza di disponibilità di cibo in quantità adeguata, sia stato risolto nel secondo dopoguerra grazie alla politica agricola comune (Pac) adottata dalla Comunità economica europea a partire dai primi anni Sessanta del secolo scorso con l'obiettivo di sostenere il reddito degli agricoltori e migliorare la produttività agri$\operatorname{cola}^{18}$. Si afferma, altresì, che la Pac abbia avuto il demerito, accanto a tanti meriti, di incentivare l'utilizzo di tecniche di allevamento e di coltivazione intensivi, con il conseguente massiccio ricorso a sostanze di derivazione chimica in agri-

17. In tema di sviluppo sostenibile si veda F. Fracchia, Lo sviluppo sostenibile. La voce flebile dell'altro tra protezione dell'ambiente e tutela della specie umana, Editoriale Scientifica, Napoli 2010. Per una sintesi dei principi in materia ambientale si veda M. Renna, I principi in materia di tutela dell'ambiente, «Rivista Quadrimestrale di Diritto dell'Ambiente», 2012, nn. $1-2$, pp. $62 \mathrm{sgg}$.

18. La politica agricola comune si basa attualmente su un pacchetto di regolamenti dell'Unione europea del 2013 di riforma della Pac per il periodo 2014-2020: nn. 1303, 1305, 1306, 1307, 1308; regolamenti successivamente modificati dal Reg. (Ue) 2017/2393. I regolamenti in parola hanno dato seguito alle strategie di intervento anticipate dalla Comunicazione della Commissione, La Pac verso il 2020: rispondere alle future sfide dell'alimentazione, delle risorse naturali e del territorio, Com(2010) 672 def. Per approfondimenti si veda I. Canfora, L. Costantino, A. Iannarelli (a cura di), Il Trattato di Lisbona e la nuova Pac, Cacucci, Bari 2017. 
Sostenibilità ambientale, sicurezza alimentare e politiche coltura e di antibiotici negli allevamenti ${ }^{19}$. La risoluzione del problema dell'insicurezza alimentare ha permesso al legislatore Ue di occuparsi degli aspetti qualitativi legati alla produzione alimentare, sviluppando una corposa legislazione in tema di igiene degli alimenti e di produzioni agroalimentari di qualità. Questa legislazione ha ricevuto un suo assetto sistematico moderno grazie al Reg. (Ce) n. 178/2002, general food law, al quale si deve la configurazione attuale del diritto alimentare in ambito europeo.

Oggi, però, si assiste alla presa di coscienza di un'insicurezza alimentare di ritorno ${ }^{20}$, risvolto negativo delle politiche ambientali di riduzione delle emissioni di CO2 nell'atmosfera. La produzione di biocarburanti e di bioplastiche di prima generazione implica l'utilizzo dei suoli agricoli per fini concorrenziali rispetto a quelli della produzione alimentare. L'utilizzo concorrenziale dei suoli agricoli - risorsa limitata - induce a riflettere sulle conseguenze future in termini di insicurezza alimentare di una produzione su larga scala di tali materiali e sostanze; senza trascurare le conseguenze ambientali legate alla deforestazione e all'utilizzo di monocolture intensive per produrre le materie prime necessarie. Lo stesso utilizzo dei suoli agricoli per l'installazione di pannelli fotovoltaici può rappresentare un uso concorrenziale degli stessi rispetto alle finalità di produzione alimentare. Questo evidenzia come sia irto di difficoltà di non poco momento e di contraddizioni il cammino verso la sostenibilità ambientale delle attività umane e come sia necessario investire risorse nella ricerca e nello sviluppo

19. Per comprendere il ruolo della Pac nel passato e le prospettive di evoluzione futura si veda la Comunicazione della Commissione, Il futuro dell'alimentazione e dell'agricoltura, 29 novembre 2017, Com(2017) 713 final. Per una riflessione critica si rinvia a S. Bolognini, La Comunicazione della Commissione europea «Il futuro dell'alimentazione e dell'agricoltura», «Rivista di diritto agrario», 2018, n. 1, pp. 110 sgg.; L. Ferraris, La protezione dell'ambiente nella Pac che verrà. Commento alla Comunicazione della Commissione sul futuro dell'alimentazione e dell'agricoltura, «Agricoltura-Istituzioni-Mercati», 2016, n. 2, pp. 175 sgg. Più in generale in argomento si possono vedere, fra gli altri, L. Costato, Le conseguenze delle trasformazioni della Pac, «Rivista di diritto agrario», 2017, n. 3, pp. 526 sgg.; Id., Per una storia della Pac (a sessant'anni dall'inserimento dell'agricoltura nel progetto di Trattato Cee), ivi, 2017, n. 1, pp. 64 sgg.; G. Follenti, Sviluppo sostenibile e agricoltura: il contributo dell'impresa agricola socialmente responsabile, «Agricoltura-Istituzioni-Mercati», 2014, n. 2, pp. $132 \mathrm{sgg}$.

20. In argomento si veda L. Costato, Dalla food security alla food insecurity, «Rivista di diritto agrario", 2011, n. 1, pp. 3 sgg; S. Amorosino, Sicurezze ed insicurezze in campo alimentare tra regolazioni e programmazioni, «Rivista di diritto alimentare», 2010, n. 3. 
di biocarburanti avanzati che non siano in concorrenza con le colture alimentari, ma che si basino, ad es., sull'utilizzo di rifiuti alimentari e scarti agricoli ${ }^{21}$.

Il richiamo alla Pac permette di sviluppare un'ulteriore riflessione collegata all'obiettivo n. 2 dell'Agenda 2030 per quanto concerne l'aumento della produttività dei suoli agricoli. Dal recente Rapporto su politica agricola comune e cambiamenti climatici realizzato da un gruppo di esperti esterni per la Commissione UE emerge la relazione conflittuale che intercorre fra le misure di sostegno alla produzione agricola contenute nella Pac e gli obiettivi di tutela ambientale, che la Pac stessa si pone. Mentre i c.d. pagamenti verdi stanziati a favore di chi adotta metodi di coltivazione rispettosi dell'ambiente - ad es. mantenimento di prati e pascoli permanenti, diversificazione delle colture, misure a tutela della biodiversità - favoriscono la riduzione delle emissioni di gas serra, al contrario gli aiuti accoppiati alla produzione, essendo legati alle rese, alle superfici delle aziende, al numero di capi allevati risultano incentivanti per gli allevamenti intensivi e, dunque, favoriscono indirettamente l'emissione di gas serra. Per questa ragione le attuali proposte relative alla Pac post 2020 (2021-2027) fissano maggiori obiettivi ambientali e climatici e prevedono una maggiore incentivazione di pratiche agricole compatibili con la tutela ambientale, rafforzandone la componente greening. La mitigazione del clima e la sostenibilità ambientale devono essere al centro della Pac, coerentemente agli obiettivi dell'Agenda 2030, al fine di rendere il settore agricolo più resiliente, sostenibile e competitivo.

Il tema della sostenibilità ambientale delle attività agricole porta a sottolineare il ruolo che svolge il metodo di coltivazione e di allevamento biologico. In questo settore il Legislatore Ue è attivo sin dal 1991 (Reg. (Cee) n. 2092/91); attualmente è ancora vigente il Reg. (Ce) n. 834/2007; dal $1^{\circ}$ gennaio 2022 troverà applicazione il

21. Lo sviluppo di biocarburanti avanzati è auspicato dall'Unione europea, consapevole dei rischi di insicurezza alimentare di ritorno derivanti dall'uso concorrenziale dei suoli agricoli; si veda, in tal senso, la direttiva 2015/1513/Ue, recepita nell'ordinamento italiano dal d.lgs. n. 51/2017. In argomento si vedano C. Losavio, La sostenibilità delle agroenergie, «Agricoltura-Istituzioni-Mercati», 2014, n. 1, pp. 83 sgg.; M. Alabrese, Il percorso dell'Ue sull'uso dei suoli per le produzioni agroenergetiche, ivi, 2013, n. 3, pp. $51 \mathrm{sgg}$. 
Sostenibilità ambientale, sicurezza alimentare e politiche

Reg. (Ue) n. 2018/848. Pur con tutti i suoi limiti, tale legislazione rappresenta uno dei più significativi strumenti messi in campo dall'Ue nell'ottica della sostenibilità ambientale, con risultati di grande interesse considerato il progressivo aumento della produzione con metodo biologico. Strumento ulteriore, meno virtuoso, ma comunque utile in un'ottica di sostenibilità ambientale è la produzione integrata: sistema di produzione agroalimentare che utilizza tutti i mezzi produttivi e di difesa delle produzioni agricole dalle avversità, volti a ridurre al minimo l'uso delle sostanze chimiche di sintesi e a razionalizzare la fertilizzazione, nel rispetto dei principi ecologici, economici e tossicologici (1. n. 4/2011). Produzione biologica e produzione integrata rappresentano due modelli alternativi alla produzione intensiva da prediligere in un'ottica di sostenibilità ambientale. Al vertice delle tecniche produttive maggiormente compatibili con la tutela dell'ambiente si pone la produzione biodinamica, che non è, però, disciplinata né dal legislatore Ue né da quello nazionale.

4. Limpegno dell'Unione europea nella lotta allo spreco alimentare

La lotta allo spreco alimentare, nel perseguimento dell'obiettivo n. 12 dell'Agenda, vede fortemente impegnato il Legislatore Ue e gli Stati membri.

Secondo i dati riportati nel documento della Commissione europea, Orientamenti dell'Ue sulle donazioni alimentari ${ }^{22}$, ogni anno nella Ue sono prodotti circa 88 milioni di tonnellate di rifiuti alimentari (173 kg pro capite annui), per un costo stimato in 143 miliardi di euro. Nello stesso tempo, nel 2015, si è calcolato che nella Ue quasi un quarto della popolazione (119,1 milioni di persone) era a rischio di povertà o di esclusione sociale e che 42,5 milioni di persone non avevano i mezzi economici necessari per permettersi un pasto di qualità un giorno su due. A livello mondiale, la Fao calcola che un terzo del cibo prodotto vada perso o sprecato, mentre 795 milioni di persone non hanno abbastanza da mangiare.

22. Commissione europea, Orientamenti dell'Ue sulle donazioni alimentari, 2017/C $361 / 01$. 
Oltre ai profili economici e sociali, lo spreco alimentare preoccupa per l'impatto ambientale. La produzione di cibo richiede l'impiego di risorse naturali, che non sono infinite. Gli alimenti che, successivamente al raccolto, vengono persi o sprecati lungo la filiera consumano circa un quarto di tutta l'acqua impiegata dall'agricoltura ogni anno e necessitano di una superficie coltivata della grandezza della Cina. Inoltre, i rifiuti alimentari generano ogni anno circa l'8\% delle emissioni globali di gas a effetto serra ${ }^{23}$.

A fronte di un quadro così desolante l'Ue ha intrapreso differenti strategie di azione ${ }^{24}$.

Nel 2016 è stata costituita la piattaforma europea contro le perdite e gli sprechi alimentari - Eu Platform on Food Losses and Food Waste, una commissione informale di esperti rappresentanti gli Stati membri, organizzazioni internazionali (tra cui Fao e Ocse), imprese alimentari e organizzazioni non governative. Il compito principale della piattaforma è di facilitare l'armonizzazione delle misure adottate dai singoli Stati e lo scambio di informazioni e buone pratiche. $\mathrm{Al}$ suo interno, alcuni gruppi studiano questioni specifiche, come l'elaborazione di un metodo affidabile e armonizzato per misurare l'ammontare dei rifiuti alimentari ${ }^{25}$.

23. Si veda anche il rapporto Fusions Reducing food waste through social innovation, Estimates of European food waste levels, 31 marzo 2016, da cui emerge che il $70 \%$ dello spreco alimentare si genera a livello domestico, nella commercializzazione al dettaglio, nella ristorazione. L'impatto dei rifiuti alimentari sull'ambiente, in particolare attraverso l'aumento della presenza di gas serra, è stato oggetto di attenzione da parte dell'Accordo di Parigi del 2015 e rappresenta un profilo di rilevante interesse nel c.d. pacchetto economia circolare dell'Unione europea. Sul punto v. M. Delsignore, Sulla necessità di una definizione armonizzata di rifuto alimentare per la concreta realizzazione dell'economia circolare, «Il diritto dell'economia», 2018, n. 2, pp. 329 sgg.

24. In proposito si veda la Comunicazione della Commissione, Il futuro sostenibile dell'Europa: prossime tappe. L'azione europea a favore della sostenibilità, 22 novembre 2016, Com(2016) 739 final, che indica le strategie di azione dell'Unione europea. Si veda, altresì, la Risoluzione del Parlamento europeo, Efficienza sotto il profilo delle risorse: ridurre lo spreco alimentare, migliorare la sicurezza alimentare, 16 maggio 2017, 2018/C 307/03. In argomento, per una sintesi delle iniziative a livello di Unione europea, si veda anche il dossier della Camera dei deputati, Recenti iniziative dell'Unione europea sull'Agenda 2030 per lo sviluppo sostenibile, 18 febbraio 2019, n. 6.

25. La piattaforma ha prodotto svariati documenti di grande interesse per il tema in esame. Si veda, in particolare, Recommendations for Actions in Food Waste Prevention, 12 dicembre 2019. La piattaforma monitora le iniziative degli Stati membri in materia di contrasto allo spreco alimentare, come risulta dal documento di sintesi sul quadro europeo Redistribution of surplus food: Examples of practices in the Member States, maggio 2019. Il monitoraggio ha riguardato anche le iniziative intraprese, sia a livello di istituzioni che di società civile, durante il periodo di emergenza sanitaria derivante dalla pandemia da Covid-19 per con- 
Sostenibilità ambientale, sicurezza alimentare e politiche
Nel 2019 la Commissione Ue ha approvato una decisione delegata riguardante una metodologia comune e requisiti minimi di qualità per la misurazione uniforme dei livelli di rifiuti alimentari ${ }^{26}$; tale iniziativa deriva dall'esigenza di poter quantificare lo spreco al fine di ridurlo. L'assenza di criteri comuni di misurazione costituisce, infatti, un ostacolo per le autorità nazionali nel comprendere la portata del problema dello spreco alimentare e la sua evoluzione. La decisione delegata precisa che, essendo i rifiuti alimentari alimenti che sono diventati rifiuti e dovendosi trarre la definizione di alimento dal Reg. (CE) n. 178/2002, i rifiuti alimentari non comprendono le perdite che si verificano in fasi della filiera alimentare in cui determinati prodotti non sono ancora diventati alimenti ai sensi dell'articolo 2 del regolamento (CE) n. 178/2002, quali piante commestibili che non sono state raccolte. Non sono, inoltre, inclusi i sottoprodotti della produzione di alimenti che soddisfano i criteri di cui all'articolo 5, paragrafo 1, della direttiva 2008/98/CE, poiché tali sottoprodotti non sono rifiuti.

Nel 2018 l'Ue ha, infine, emanato un pacchetto di direttive (c.d. pacchetto economia circolare o pacchetto rifiuti) entrato in vigore a luglio di quello stesso anno e da recepire entro luglio $2020^{27}$, di cui fa parte la direttiva (UE) 2018/851, che modifica la direttiva quadro sui rifiuti 2008/98/CE, inserendo fra i rifiuti anche quelli alimentari ${ }^{28}$. Rifiuti alimentari sono tutti gli alimenti, tali ai sensi dell'art. 2 Reg. (CE)

tenere lo spreco alimentare; si veda, a questo proposito, la newsletter di marzo 2020, Food waste prevention initiatives during the Covid-19 crisis. Si ricorda, con riguardo a tale emergenza sanitaria, che l'Unione europea ha emanato il Reg. (Ue) 2020/466 recante misure temporanee volte a contenere rischi sanitari diffusi per l'uomo, per gli animali e per le piante e per il benessere degli animali in occasione di determinate gravi disfunzioni dei sistemi di controllo ufficiale sulla filiera agroalimentare degli Stati membri dovute alla malattia da coronavirus (Covid-19). A propria volta Fao ed Oms hanno emanato il documento Covid-19 and Food Safety: Guidance for Food Businesses: interim guidance, 7 aprile 2020.

26. Decisione delegata (Ue) 2019/1597 della Commissione del 3 maggio 2019 che integra la direttiva 2008/98/CE del Parlamento europeo e del Consiglio per quanto riguarda una metodologia comune e requisiti minimi di qualità per la misurazione uniforme dei livelli di rifiuti alimentari.

27. Per quanto riguarda l'ordinamento italiano si veda T. Ronchetti, M. Medugno, Pacchetto economia circolare: al via il recepimento, "Ambiente e sviluppo», 2020, n. 4, pp. 279 sgg.

28. In argomento si veda M. Cocconi, La regolazione dell'economia circolare. Sostenibilità e nuovi paradigmi di sviluppo, FrancoAngeli, Milano 2020; M. D'Addezio, Transizione verso un'economia circolare: diritto per l'agricoltura e coordinate costituzionali. Prime riflessioni, «Rivista di diritto agrario», 2017, n. 1, pp. 85 sgg.; F. De Leonardis, Economia circolare: saggio 
n. $178 / 2002$, che sono diventati rifiuti. Si ricorda che per rifiuto si intende, sul piano giuridico, «qualsiasi sostanza o oggetto di cui il detentore si disfi o abbia l'intenzione o l'obbligo di disfarsi». La direttiva, attraverso la definizione di rifiuto alimentare, supera le difficoltà interpretative legate alla distinzione fra food losses (perdite alimentari) e food waste (rifiuti alimentari) e fornisce una formula unitaria che può essere intesa come sintesi di armonizzazione per la definizione di spreco alimentare ${ }^{29}$.

La direttiva (Ue) 2018/851 impone agli Stati membri di controllare la produzione di rifiuti alimentari e di adottare misure per limitarla. Fra le misure vi sono incentivi per incoraggiare la donazione di alimenti e altre forme di distribuzione delle eccedenze nonché campagne di sensibilizzazione. L'obiettivo è quello di giungere ad una riduzione del $30 \%$ dei rifiuti alimentari entro il 2025 e del $50 \%$ entro il 2030, come richiesto dall'obiettivo n. 12 dell'Agenda 2030. Lo Stato italiano deve recepire la direttiva modificando il codice dell'ambiente d.lgs. n. 152/2016.

Fra le iniziative dell'Ue per la riduzione dello spreco alimentare si ricorda la campagna di sensibilizzazione dei consumatori per l'adozione di buone pratiche e per la migliore comprensione della differenza fra data di scadenza (da consumarsi entro - use by) e termine minimo di conservazione (da consumarsi preferibilmente entri - best before) ${ }^{30}$.

Infine, si ricorda la già menzionata comunicazione della Commissione, Orientamenti dell'Ue sulle donazioni alimentari finalizzata a dissipare i dubbi sui profili legislativi delle donazioni di alimenti, fissando linee comuni in materia di sicurezza alimentare, rintracciabilità, responsabilità, fiscalità da parte di chi dona o riceve cibo.

sui suoi tre diversi aspetti giuridici. Verso uno Stato circolare?, «Diritto amministrativo», 2017, pp. 163 sgg.

29. In merito alla distinzione fra food losses, concetto riferito alle perdite che si verificano nelle fasi della produzione agricola, della post-raccolta, della trasformazione ed elaborazione degli alimenti, e food waste, riferibile ai rifiuti che si generano nell'ultima fase della filiera alimentare caratterizzata dalla commercializzazione al dettaglio, dalla somministrazione e dal consumo, si veda E. Varese (a cura di), Sprechi alimentari: una prospettiva multidisciplinare. Consumatori e imprese a confronto, Giappichelli, Torino 2015. Per ulteriori approfondimenti sul tema si veda altresì G. Maccioni, Spreco alimentare. Regole e limiti nella transizione verso modelli agroalimentari sostenibili, Giappichelli, Torino 2018; L. Costantino, La problematica degli sprechi nella filiera agroalimentare, Cacucci, Bari 2018.

30. https://ec.europa.eu/food/safety/food_waste/stop_en. 
Sostenibilità ambientale, sicurezza alimentare e politiche
Il documento da ultimo menzionato sottolinea che, per prevenire gli sprechi, è necessario intervenire alla fonte, limitando la produzione di eccedenze alimentari in ciascuna fase della filiera (produzione, trasformazione, distribuzione e consumo). Laddove vi siano eccedenze, la migliore destinazione possibile, in presenza di prodotti alimentari ancora idonei al consumo, è la ridistribuzione per l'alimentazione umana. La ridistribuzione alimentare è un processo grazie al quale eccedenze alimentari, che potrebbero altrimenti andare sprecate, vengono recuperate, raccolte e fornite a persone bisognose.

Le eccedenze alimentari, costituite da prodotti alimentari finiti (compresi carne, frutta e ortaggi freschi), prodotti parzialmente formulati o ingredienti alimentari, possono generarsi - sottolinea il documento in esame - in qualsiasi fase della filiera di produzione e distribuzione degli alimenti, per motivi di varia natura. Eccedenze nei settori agricolo e manifatturiero possono generarsi con riguardo ad alimenti non conformi alle specifiche del produttore e/o del cliente (ad esempio differenze di colore, dimensione, forma del prodotto ecc.) oppure per errori di produzione e di etichettatura. Difficoltà nella gestione dell'offerta e della domanda possono dare luogo a ordinazioni eccedentarie e/o ad annullamenti di ordini.

La Commissione sottolinea come problemi connessi all'indicazione della data di consumo - quali un'insufficiente vita residua dei prodotti al momento della consegna oppure la presenza di norme nazionali che impediscono la ridistribuzione degli alimenti successivamente al termine minimo di conservazione, consistente nella data indicata nella dicitura «da consumarsi preferibilmente entro il»-possono precludere la vendita e la distribuzione di generi alimentari attraverso i consueti canali della vendita al dettaglio. A questo proposito il documento della Commissione si sforza di apportare chiarezza relativamente ad un profilo oggetto di ricorrente incertezza; trattasi della liceità della commercializzazione di alimenti oltre lo spirare del termine minimo di conservazione ${ }^{31}$. A tal proposito 
si afferma che la commercializzazione degli alimenti oltre il termine minimo di conservazione è consentita ai sensi delle norme dell'UE, a condizione che i prodotti interessati siano ancora sicuri e la loro presentazione non induca in errore. In ogni fase della filiera alimentare è, quindi, consentito immettere sul mercato prodotti che hanno superato il termine minimo di conservazione. Spetta all'operatore del settore alimentare (ad esempio il dettagliante) garantire che l'alimento sia ancora sicuro per il consumo umano e che i consumatori siano debitamente informati del fatto che il prodotto interessato ha superato la data di cui alla dicitura «da consumarsi preferibilmente entro il»; i prodotti in questione potrebbero, ad es., essere commercializzati separatamente, con l'indicazione dell'avvenuto superamento del termine minimo di conservazione. Risulta evidente che eventuali discipline nazionali che vietano la commercializzazione di alimenti con termine minimo di conservazione superato favoriscono sprechi alimentari evitabili; si può notare, a questo specifico riguardo, che il legislatore italiano non ha ancora preso esplicita posizione, in termini generali, sulla possibilità di commercializzazione di alimenti con termine minimo di conservazione superato, generando in tal modo incertezza e cautela fra gli operatori del settore.

Ai fini dell'applicazione della legislazione in materia di food safety è irrilevante il fine di lucro connesso alla fornitura a terzi di alimenti; per tale ragione anche la distribuzione di alimenti a fine di beneficenza è soggetta agli obblighi di sicurezza alimentare previsti dal Reg. (Ce) n. 178/2002 e dal c.p. pacchetto igiene con riguardo, in particolare, al Reg. (Ce) n. 852/2004 nonché agli obblighi di etichettatura di cui al Reg. (Ue) n. 1169/2011. Principio cardine, da

data di scadenza sia riservata agli alimenti molto deperibili dal punto di vista microbiologico che potrebbero, quindi, costituire, dopo un breve periodo, un pericolo immediato per la salute umana. Successivamente allo spirare della data di scadenza l'alimento è considerato a rischio e non può più essere immesso in commercio. Sul piano dell'ordinamento interno si ricorda il d.lgs. n. 231/2017 che reca la disciplina sanzionatoria per le violazioni agli obblighi di etichettatura di cui al Reg. (Ue) n. 1169/2011; l'art. 12 detta la disciplina sanzionatoria relativa alla violazione del divieto di immissione in commercio di alimenti con data di scadenza superata. Si può notare che non vi sia un divieto espresso, invece, per la immissione in commercio di alimenti con termine minimo di conservazione superato; in tal caso l'alimento non può, infatti, essere considerato a priori come non sicuro poiché il termine minimo di conservazione indica il periodo in cui sono garantite, in condizioni idonee di conservazione, le qualità organolettiche specifiche dell'alimento. 
Sostenibilità ambientale, sicurezza alimentare e politiche questo punto di vista, è quello secondo cui tutti i consumatori devono essere tutelati allo stesso modo, dalle stesse norme di sicurezza alimentare. Di conseguenza, rientrano nella nozione di operatore del settore alimentare, primo responsabile della sicurezza degli alimenti immessi in commercio, anche le organizzazioni che ricevono le eccedenze alimentari, siano esse organizzazioni di ridistribuzione o di beneficienza.

Le eccedenze alimentari possono essere ridistribuite a condizione che siano idonee al consumo umano e conformi a tutti i requisiti di sicurezza alimentare imposti dalle norme dell'Ue in materia di sicurezza degli alimenti e di informazioni alimentari ai consumatori e dalle norme nazionali pertinenti. Tra gli alimenti idonei alla donazione possono figurare, ad esempio, prodotti che: non rispettano le specifiche del produttore o del cliente; presentano alterazioni a livello di imballaggio e/o di etichettatura che tuttavia non compromettono né la sicurezza dell'alimento né l'informazione al consumatore; recano una indicazione di carattere temporale (prodotti destinati a un particolare periodo di festa o a una particolare attività promozionale); sono raccolti nei campi con il consenso del produttore; hanno superato la data indicata nella dicitura «da consumarsi preferibilmente entro il» ma possono ancora essere consumati in sicurezza; sono stati raccolti e/o confiscati dalle autorità di regolamentazione per motivi non attinenti alla sicurezza alimentare ecc.

Gli operatori del settore alimentare possono dunque ridistribuire le eccedenze alimentari e svolgere attività di donazione in ogni fase della filiera. Tali operatori (ad esempio agricoltori, produttori e dettaglianti di generi alimentari) possono donare gli alimenti in eccesso tramite organizzazioni di ridistribuzione (come le banche alimentari), reti di raccolta e altre organizzazioni di beneficenza oppure elargirli direttamente agli stessi consumatori (ad esempio il personal dipendente).

\section{I riflessi nell'ordinamento italiano: la l. $n$. 166/2016}

L'ordinamento italiano si caratterizza, sul piano del contrasto allo spreco alimentare, per aver preso l'iniziativa di incentivare le donazioni delle eccedenze alimentari attraverso la l. n. 166/2016, recante disposizioni concernenti la 
donazione e la distribuzione di prodotti alimentari e farmaceutici a fini di solidarietà sociale e per la limitazione degli sprechi ${ }^{32}$.

Per eccedenze alimentari si intendono i prodotti alimentari, agricoli e agro-alimentari che sono invenduti, non somministrati per carenza di domanda, ritirati dalla vendita perché non conformi ai requisiti aziendali di vendita, rimanenze di attività promozionali, prossimi al raggiungimento della data di scadenza, rimanenze di prove di immissione in commercio di nuovi prodotti, invenduti a causa di danni provocati da eventi meteorologici, invenduti a causa di errori nella programmazione della produzione, non idonei alla commercializzazione per alterazioni dell'imballaggio secondario che non inficiano le idonee condizioni di conservazione. L'elenco di situazioni che determinano un'eccedenza alimentare è presentato dalla legge stessa come non esaustivo dimostrando l'intenzione, da parte del legislatore, di essere il più onnicomprensivo possibile; resta fermo, però, che devono essere rispettati e mantenuti i requisiti di igiene e sicurezza del prodotto.

Il legislatore si premura di stabilire a quale accezione di spreco alimentare intenda fare riferimento; assume rilevanza, a tal fine, la circostanza che il prodotto alimentare sia stato scartato dalla catena agroalimentare per ragioni commerciali o estetiche o per prossimità della scadenza e che il medesimo sia ancora commestibile e potenzialmente destinabile al consumo umano o animale e che, in assenza di un utilizzo alternativo, sia destinato ad essere smaltito.

32. Per un approfondimento, anche in chiave critica, sulla l. n. 166/2016 e per un confronto con la coeva 1. francese n. 2016-138 dell'11 febbraio 2016, finalizzata alla lutte contre le gaspillage alimentaire, si vedano E. Ferrero, Sistema alimentare ed economia circolare, "Ambiente e sviluppo», 2018, n. 7, pp. 471 sgg.; G. Spoto, La cessione a titolo gratuito delle eccedenze alimentari contro gli sprechi, "Europa e diritto privato», 2017, pp. 1111 sgg.; I. Trapè, Lo spreco alimentare e la legge italiana n. 166 del 2016, "Rivista di Diritto agrario», 2017, n. 2, pp. 263 sgg.; A. Gorassini, Il cibo come bene comune. Periplo di una recente legge con tentativo di approdo, "Jus civile», 2017, n. 5, pp. 433 sgg.; F. Pepe, Approvata la legge contro lo spreco alimentare, «Rivista di diritto alimentare», 2016, n. 3, pp. 56 sgg. Per uno sguardo anche a livello regionale si veda L. Costantino, La sostenibilità della filiera agroalimentare nell'ottica dell'economia circolare, «Agricoltura-Istituzioni-Mercati», 2017, n. 1, pp. 5 sgg. Per un quadro complessivo delle iniziative degli Stati membri al fine di contrastare lo spreco alimentare si può vedere il già richiamato documento Eu Platform on Food Losses and Food Waste, Redistribution of surplus food: Examples of practices in the Member States, maggio 2019. 
Sostenibilità ambientale, sicurezza alimentare e politiche
Costituisce, perciò, spreco alimentare l'insieme dei prodotti alimentari aventi le caratteristiche indicate.

La finalità della 1. n. 166/2016, come anticipato, è quella di incentivare gli operatori del settore alimentare a donare, cedendole gratuitamente a soggetti donatari, le eccedenze alimentari promuovendo, in tal modo, un fine di solidarietà sociale in un'ottica di sussidiarietà orizzontale ${ }^{33}$.

Le norme che disciplinano le modalità di cessione consentono che siano ceduti anche gli alimenti che hanno superato il termine minimo di conservazione - ovvero, come già ricordato, il termine di durabilità che non incide sulla sicurezza del prodotto bensì sulle proprietà organolettiche specifiche dell'alimento - purché sia garantita l'integrità dell'imballaggio primario e le idonee condizioni di conservazione. Possono essere ceduti anche alimenti che presentino irregolarità di etichettatura che non riguardano le informazioni relative alla data di scadenza oppure alla presenza di allergeni alimentari. Ugualmente consentita è la cessione a titolo gratuito delle eccedenze di prodotti agricoli in campo o di prodotti di allevamento idonei al consumo umano ed animale ai soggetti donatari. In tal caso le operazioni di raccolta o ritiro dei prodotti agricoli possono essere effettuate direttamente dai soggetti donatari sotto la loro responsabilità, nel rispetto delle norme in materia di igiene e sicurezza alimentare

I soggetti donatari devono destinare, in forma gratuita, le eccedenze alimentari ricevute, che siano idonee al consumo umano, prioritariamente a persone indigenti; le eccedenze non idonee al consumo umano possono essere destinate al consumo animale, al compostaggio di comunità con metodo aerobico, all'autocompostaggio ${ }^{34}$. Si prevede, altresì, che le eccedenze alimentari possano essere ulterior-

33. L'incentivo è collegato a sgravi fiscali; si veda, in particolare, l'art. 17 della 1 . n. 166/2016 che prevede la possibilità per i Comuni di ridurre la tariffa relativa alla tassa sui rifiuti a favore delle utenze non domestiche relative ad attività commerciali, industriali, professionali e produttive in genere, che producono o distribuiscono beni alimentari, e che a titolo gratuito cedono, direttamente o indirettamente, tali beni alimentari agli indigenti e alle persone in maggiori condizioni di bisogno ovvero per l'alimentazione animale; il coefficiente di riduzione della tariffa deve essere proporzionale alla quantità, certificata, di beni e prodotti ritirati dalla vendita e oggetto di donazione. 34. Si veda, in proposito, la Comunicazione della Commissione, Orientamenti per l'utilizzo come mangimi di alimenti non più destinati al consumo umano, 2018/C 133/02. 
mente trasformate in prodotti destinati, in via prioritaria, all'alimentazione umana o al sostegno vitale degli animali; resta fermo il rispetto dei requisiti di igiene e della data di scadenza. Si consente, infine, che i prodotti finiti della panificazione e i derivati degli impasti di farina prodotti negli impianti di panificazione, non necessitanti di condizionamento termico, che, non essendo stati venduti o somministrati entro le ventiquattro ore successive alla produzione, risultano eccedenti presso le rivendite di negozi, anche della grande distribuzione, i produttori artigianali o industriali, la ristorazione organizzata, inclusi gli agriturismi, e la ristorazione collettiva, possano essere donati a soggetti donatari.

In linea con gli orientamenti dell'Unione europea, che ritiene fondamentale sensibilizzare i consumatori a adottare comportamenti più consapevoli sul fronte dello spreco alimentare, la 1. n. 166/2016 delinea uno scenario di promozione, formazione e adozione di misure preventive con il coinvolgimento del servizio pubblico radiofonico, televisivo, multimediale, dei ministeri delle politiche agricole, della salute, dell'ambiente. Particolare attenzione è rivolta al settore della ristorazione, ambito in cui la prevenzione dello spreco implica l'adozione di pratiche virtuose che consentano, ad es., ai clienti l'asporto, in sicurezza, dei propri avanzi di cibo.

Fondamentale nella diffusione di una cultura improntata al rispetto per il cibo è, infine, il ruolo delle istituzioni scolastiche presso le quali dovrebbero essere promossi percorsi mirati all'educazione ad una sana alimentazione, ad una produzione alimentare ecosostenibile e alla sensibilizzazione contro lo spreco degli alimenti ${ }^{35}$.

La l. n. 166/2016 ha, dunque, adottato una linea «morbida» per contrastare lo spreco alimentare, basata sull'introduzione di un regime incentivante, sul piano fiscale e de-

35. L'importanza dell'educazione sin dall'infanzia nel contrasto allo spreco è sottolineata dalla già richiamata Risoluzione del Parlamento europeo, Efficienza sotto il profilo delle risorse: ridurre lo spreco alimentare, migliorare la sicurezza alimentare, del 16 maggio 2017, in cui si sottolinea anche l'importanza di un efficace sistema di controllo da parte della Commissione e degli Stati per impedire che attraverso le donazioni delle eccedenze alimentari si crei un mercato alternativo tale da escludere i soggetti bisognosi e da scoraggiare i professionisti del settore dall'effettuare donazioni per evitare rischi di concorrenza sleale. In argomento si veda anche R.M. Fanelli, A. Di Florio, Lo spreco alimentare nella fase del consumo domestico, «Agricoltura-Istituzioni-Mercati», 2013, n. 3, pp. 33 sgg. 
Sostenibilità ambientale, sicurezza alimentare e politiche gli adempimenti burocratici, la donazione delle eccedenze alimentari; la scelta del legislatore italiano si è orientata in maniera differente rispetto a quella del legislatore francese che, nello stesso anno, ha invece optato per una linea «dura» basata sull'obbligo, sanzionato con pene detentive e pecuniarie, di recupero e redistribuzione delle eccedenze alimentari in capo ai soggetti della grande distribuzione organizzata.

Concludendo, si può affermare che l'obiettivo dello sviluppo sostenibile declinato con riguardo agli alimenti imponga un radicale mutamento culturale che coinvolge tutti i livelli della società civile, chiamando in causa il principio di sussidiarietà orizzontale, e che, per essere realmente efficace, deve seguire un processo bottom up attraverso la presa di coscienza da parte della collettività dell'importanza di avere «rispetto» per il cibo, in ragione delle risorse naturali, umane e materiali impiegate per produrlo e delle disuguaglianze a livello globale sulla distribuzione di alimenti in quantità adeguata ed in idonee condizioni igieniche. A tal fine occorrerebbe anche recuperare il senso del «sacrificio» inteso come disponibilità ad impegnare tempo, energie personali e risorse economiche per gestire il rapporto con il cibo in modo più consapevole. 
I rifiuti sono il risultato di un processo di consumo.

Ogni processo, anche naturale produce rifiuti, scarti. La coevoluzione degli organismi nel corso dello sviluppo della vita sulla terra ha portato allo sviluppo di cicli basati sull'utilizzo di uno scarto di un ciclo per alimentarne un altro.

D’altro canto, però, un processo è via via più efficiente, quanti meno scarti produce, massimizzando la trasformazione dei materiali in ingresso.

Oggi l'uomo si sta rivelando la specie vivente che accumula più rifiuti senza che nel frattempo si siano evolute altre specie in grado di metabolizzarne gli scarti. Ciò è dovuto da un lato alla velocità di accumulo, dall'altro dalla sintesi di molecole nuove.

Per tale ragione le norme più recenti si ispirano alla natura cercando di spostare l'economia da un approccio lineare ad uno circolare.

In particolare, il pacchetto di direttive comunitarie sull'economia circolare dell'Unione Europea prevede, tra diverse misure, il dimezzamento degli sprechi alimentari globali pro capite entro il 2030. 
Consumo responsabile e lotta allo spreco
Sono escluse dall'ambito di applicazione della direttiva tutte le sostanze destinate ad essere utilizzate come materie prime per mangimi.

Il testo che segue è estratto dal capitolo 3 de "La Bibbia dell'Ecologia” pubblicato per EllediCi editrice nel $2018^{1}$.

1. Gli sprechi alimentari

Stando agli ultimi dati pubblicati dall'ISTAT, relativi all'annata agraria del 2011, circa il 2\% del prodotto resta in campo. Ho provato a selezionare le coltivazioni più diffuse in Italia e ad elaborare i dati di produzione e raccolta.

Le spighe, che mio nonno mi faceva raccogliere, sono relativamente poche: solo lo $0,74 \%$ resta in campo. Ma se guardiamo agli ortaggi coltivati in serra la percentuale che viene sprecata supera il $3,5 \%$ !

Perdite medie in campo delle principali coltivazioni in Italia

\begin{tabular}{|ccccc|}
\hline coltivazione & produzione tot (q) & produrione raccolta (q) & Perdite (q) & perdite in \% \\
\hline Cereali & $181.610 .791,00$ & $180.267 .202,00$ & $1.343 .589,00$ & $0,74 \%$ \\
\hline Legumi secchi & $1.343 .165,00$ & $1.319 .913,00$ & $23.252,00$ & $1,73 \%$ \\
\hline Piante da tubero (patate) & $16.045 .773,00$ & $15.676 .844,00$ & $368.929,00$ & $2,30 \%$ \\
\hline Ortaggi in piena aria & $122.379 .688,00$ & $118.553 .251,00$ & $3.826 .437,00$ & $3,13 \%$ \\
\hline Ortaggi in serra & $16.394 .047,00$ & $15.807 .919,00$ & $586.128,00$ & $3.5 \% \%$ \\
\hline Frutta fresca & $62.289 .120,00$ & $60.954 .062,00$ & $1.335 .058,00$ & $2,14 \%$ \\
\hline Agrumi & $39.145 .800,00$ & $38.360 .665,00$ & $785.135,00$ & $2,01 \%$ \\
\hline Vite & $74.868 .128,00$ & $72.828 .700,00$ & $2.039 .428,00$ & $2,72 \%$ \\
\hline TOATALE & $514.076 .512,00$ & $503.768 .556,00$ & $10.307 .956,00$ & $2,01 \%$ \\
\hline
\end{tabular}

(Rielaborazione ERICA su dati ISTAT, 2011).

Se consideriamo il valore medio dei vari prodotti il dato è impressionante.

Difficile fare una media, ma pensando che il frumento si paga tra i 200 e i 270 euro a tonnellata, il granoturco tra i 180 e i 240 euro a tonnellata, l'orzo tra i 200 e i 260 , il sorgo tra i 175 e i 275 euro a tonnellata, possiamo stimare il valore medio dei cereali, al ribasso, attorno ai 200 euro a tonnellata, ovvero 20 euro a quintale. Con lo stesso principio, per le patate all'ingrosso possiamo assumere un valore 
di 25 euro al quintale, e per gli ortaggi 70 euro al quintale, dal minimo delle cipolle a 25 euro al quintale ai pomodorini ciliegina da 185 euro al quintale, e così via.

Perdite medie in campo delle principali coltivazioni in Italia

\begin{tabular}{|ccccc|}
\hline coltivazione & produzione tot (q) & produzione raccolta (q) & Perdite (q) & perdite in \% \\
\hline Cereali & $181.610 .791,00$ & $180.267 .202,00$ & $1.343 .589,00$ & $0,74 \%$ \\
\hline Legumi secchi & $1.343 .165,00$ & $1.319 .913,00$ & $23.252,00$ & $1,73 \%$ \\
\hline Piante da tubero (patate) & $16.045 .773,00$ & $15.676 .844,00$ & $368.929,00$ & $2,30 \%$ \\
\hline Ortaggi in piena aria & $122.379 .688,00$ & $118.553 .251,00$ & $3.826 .437,00$ & $3,13 \%$ \\
\hline Ortaggi in serra & $16.394 .047,00$ & $15.807 .919,00$ & $586.128,00$ & $3.58 \%$ \\
\hline Frutta fresca & $62.289 .120,00$ & $60.954 .062,00$ & $1.335 .058,00$ & $2,14 \%$ \\
\hline Agrumi & $39.145 .800,00$ & $38.360 .665,00$ & $785.135,00$ & $2,01 \%$ \\
\hline Vite & $74.868 .128,00$ & $72.828 .700,00$ & $2.039 .428,00$ & $2,72 \%$ \\
\hline TOATALE & $514.076 .512,00$ & $503.768 .556,00$ & $10.307 .956,00$ & $2,01 \%$ \\
\hline
\end{tabular}

(Rielaborazione ERICA su dati ISTAT, 2011).

Quindi solo in Italia, con i prodotti rimasti in campo, avremmo nel portafoglio quasi 700 milioni di euro all'anno, senza nemmeno aver pagato per produrre tutto questo bendidio. A livello mondiale la stima del valore delle perdite in campo potrebbe aggirarsi attorno ai 43 miliardi di dollari.

In pratica, stando alla classifica stilata ogni anno da Forbes $^{2}$, saremmo, nel 2016, attorno al settimo posto tra gli uomini «più ricchi della terra», subito dietro a Mark $\mathrm{Zu}$ ckerberg, fondatore di Facebook.

Purtroppo, però ciò che resta nei campi non può trasformarsi in banconote da piegare nel portafoglio, come mi diceva mio nonno.

A parte che non ci starebbe in nessun portafoglio, ma soprattutto non si conserva, proprio come la manna del deserto dell'Esodo.

Esodo 16, 3-203:

Al mattino poi, intorno all'accampamento, c'era uno strato di rugiada. Essa sparì e sulla superficie del deserto, per terra, rimase una cosa fine e granulosa, fine come la brina. Gli Israeliti la videro e non sapevano che cosa era. Perciò si chiedevano l'un l'altro: «Che cos'è?».

2. forbes.com, marzo 2017.

3. Vedi anche Numeri 11, 7-9 e Giosuè 5, 10-12. 
Consumo responsabile e lotta allo spreco
Mosè disse loro: «È il pane che il Signore vi ha dato da mangiare. Ed egli vi comanda di raccoglierne quanto ciascuno può mangiarne: la misura di un omer a testa, secondo il numero delle persone che sono con voi. Ciascuno ne prenda per quelli della sua tenda».

Gli Israeliti fecero così: alcuni ne raccolsero molto, altri poco. Quando si misurò la quantità, si vide che chi ne aveva raccolto molto, non ne aveva più degli altri, mentre chi ne aveva raccolto poco, ne aveva a sufficienza. Ciascuno ne aveva quanto poteva mangiarne.

Poi Mosè disse loro: «Non dovete farne avanzare fino a domani mattina!». Alcuni però non gli ubbidirono e ne conservarono un po' fino al mattino seguente; ma vi nacquero dentro dei vermi e marcì. Mosè andò in collera contro quelle persone.

Abbastanza. Che ti sia sufficiente. Che tu ne possa mangiare oggi, perché domani ne avrai ancora. Senza sprecare. Quanto è moderna questa riflessione! Quanto è moderno l'invito di Mosè: inascoltato allora e ancor di più oggi. Lo spreco alimentare, infatti, non si ferma al campo, ma prosegue lungo tutta la catena di distribuzione e consumo, fino al nostro frigorifero di casa.

La Commissione Europea ${ }^{4}$ classifica gli sprechi a seconda delle fasi:

- manufacturing: i processi di trattamento e trasformazione dei prodotti alimentari destinati alla distribuzione;

- retail/wholesale: le attività di distribuzione e vendita a individui o organizzazioni;

- food service sector: catering e ristorazione;

- households: consumo famigliare.

In Europa, ogni anno, la quantità di cibo che viene sprecato è di circa 90 milioni di tonnellate, in pratica 180 chili a testa. La quantità maggiore di questo spreco, 76 chili all'anno, è nelle nostre case, dopo che abbiamo fatto la spesa $^{5}$. In Italia questo valore è addirittura più alto: but-

4. Commissione Europea - DG Ambiente Unità C1, Food Waste in the EU: a study by the European Commission, Workshop on Municipal Waste Prevention, Barcellona, 24 novembre 2011.

5. Barilla Center for Food and Nutrition, Lo spreco alimentare: cause, impatti e proposte, giugno 2012. 
tiamo nella spazzatura più di 100 chili di cibo per persona all'anno ${ }^{6}$.

Questo spreco ha numerose ripercussioni. Certamente genera un danno economico, come già suggeriva mio nonno, ma genera anche disagi sociali e impatti ambientali. Per completare il ragionamento economico, se i prodotti rimasti in campo, solo in Italia, per un solo anno, hanno un valore stimabile attorno ai 700 milioni di euro, l'intero spreco alimentare assomma a 10 miliardi di euro!

\section{Gli impatti ambientali degli sprechi alimentari}

Dal punto di vista ambientale occorre considerare che la produzione di cibo richiede acqua, energia, fitofarmaci.

Per calcolare l'impatto ambientale degli sprechi alimentari si utilizzano normalmente tre indicatori:

- l'impronta di carbonio (carbon footprint);

- l'impronta ecologica (ecological footprint);

- l'impronta idrica (water footprint).

Limpronta di carbonio stima la somma di tutte le emissioni di gas che possono avere un effetto climalterante, ovvero di quei gas, cosiddetti ad effetto serra, che vengono liberati in atmosfera nelle varie fasi di ottenimento di un prodotto. In agricoltura e nelle successive trasformazioni, fino all'ottenimento di un cibo per l'uomo, le emissioni derivano dal consumo di carburanti per le lavorazioni del terreno, per i trasporti, per la produzione degli imballaggi, per mantenere i cibi al freddo. Altre emissioni sono originate dagli allevamenti, in particolare, dal biogas, con prevalente presenza di metano, derivante dalle deiezioni animali. Occorre considerare che il metano ha un potere di modificare il clima tra le 21 e le 25 volte superiore al CO2.

Ci sono poi le emissioni dei fertilizzanti a base di azoto che tende a liberarsi in atmosfera sotto forma di protossido di azoto (N2O). L'impronta ecologica, come ho avuto modo di descrivere in altro capitolo, è la quantità di superficie di terra e mare necessaria a produrre le risorse indispensabili per ottenere un determinato prodotto o ospitare una

6. Andrea Segrè, Luca Falasconi, Il libro nero dello spreco in Italia: il cibo, Edizioni Ambiente, Milano 2011. 
Consumo responsabile e lotta allo spreco

certa popolazione ed assorbirne i relativi rifiuti prodotti. Lo stesso indicatore, anziché in termini di superficie, può essere sviluppato in volume e in peso: è ciò che hanno fatto i ricercatori del Wuppertal Institut ${ }^{7}$ definendo lo «zainetto ecologico» (Ecological Rucksack) ${ }^{8}$.

In pratica quando acquistiamo o consumiamo o buttiamo un oggetto, ciò che abbiamo tra le mani ha un peso ecologico molto superiore al reale peso sulla bilancia. Lo zaino ecologico infatti porta con sé i chili di natura estratti e utilizzati e in gran parte buttati via per realizzare un bene.

E poi c'è la quantità d'acqua utilizzata nel processo di ottenimento di un bene. Quando studiavo alla Scuola Enologica di Alba il professore di economia ci insegnava come nel bilancio di una cantina occorresse considerare un consumo di acqua pari al doppio del vino prodotto: e non per allungar- lo, ma solo per le operazioni di lavaggio delle attrezzature e dei locali.

Limpronta idrica (Water Footprint o Virtual water content) calcola non solo la quantità di acqua dolce utilizzata, ma anche come l'acqua è consumata; ad esempio, nel caso dell'ottenimento di alimenti viene considerata sia l'acqua usata per irrigare le coltivazioni sia quella di- spersa attraverso l'evaporazione del terreno e delle piante, fino all'acqua consumata nelle fasi di trasformazione, confezionamento, trasporto e vendita del prodotto finale.

Il centro studi della Barilla per il cibo e la nutrizione (Bcfn) ha elaborato una serie di dati forniti dal Ministero della Pesca e dell'Agricoltura della Danimarca fornendo calcoli precisi sull'impatto ambientale dei cibi. Ad esempio, la preparazione di un chilo di sogliola fresca, con i vari passaggi dalla pesca al confezionamento, genera $3,3 \mathrm{~kg}$ di CO2; analogamente la preparazione di un kg di gamberi surgelati produce $10 \mathrm{~kg}$ di emissioni di CO2 e ancora un $\mathrm{kg}$ di aragosta produce $20 \mathrm{~kg}$ di CO2! Lo studio arriva a classificare i cibi ponendoli su diversi livelli, che formano una doppia piramide: una è quella classica alimentare e l'altra, invertita, è quella ambientale.

7. wupperinst.org, novembre 2017.

8. ressourcen-rechner.de, giugno 2017. 


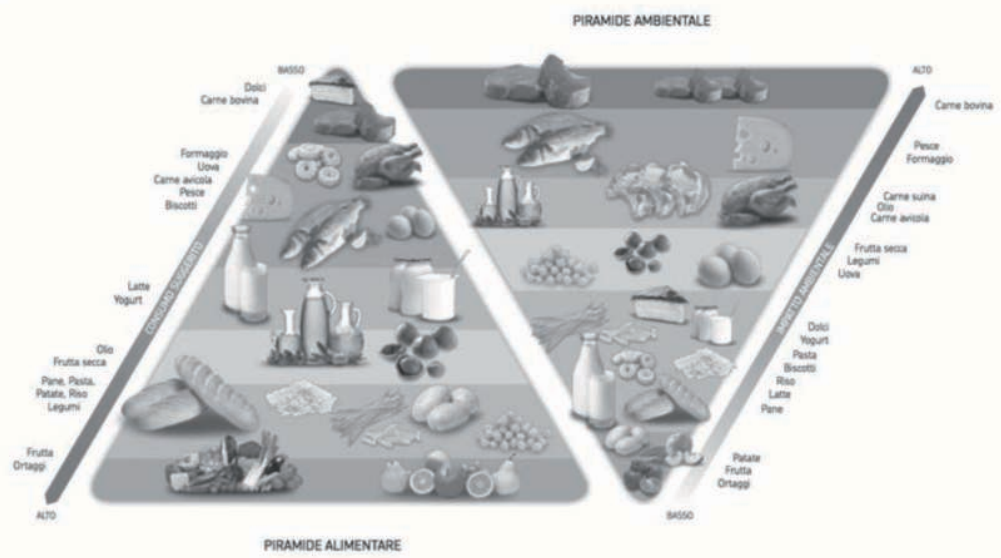

(Fonte: Barilla Center for Food \& Nutrition, 2015).

All'interno di ogni livello ci sono cibi con valori nutrizionali analoghi e impatto ambientale equiparabile. Consultando la piramide alimentare e quella ambientale possiamo orientare la nostra scelta alimentare a parità di kcal verso il minor impatto ambientale.

Ad esempio, per ottenere un chilo di formaggio servono 5.000 litri di acqua e si emettono $9 \mathrm{~kg}$ di CO2 mentre per una quantità di yogurt equivalente dal punto di vista alimentare, si emette un solo chilo di $\mathrm{CO} 2$ e si consuma cinque volte meno di acqua.

I dati sono ancora relativamente approssimativi, soprattutto per alcuni cibi, basti pensare alle diverse specie di pesce, ma anche per la complessità di analisi e comparazione dei parametri e relativi valori, come ad esempio la diversa origine dell'energia a seconda che lo stesso cibo sia prodotto in Svezia (energia dal vento e dall'acqua) o in Francia (energia nucleare) o in Italia (energia dal gas metano).

La piramide è costruita per classi di alimenti, senza valori puntuali e, anche se nei prossimi anni si avranno dati più precisi, l'attuale distribuzione tra $\mathrm{i}$ diversi livelli non dovrebbe cambiare.

Ad esempio «le patate, per cui ora è stimata un'impronta ecologica media di sette metri quadrati, compresa la cottura, 
Consumo responsabile e lotta allo spreco potranno alzarsi o abbassarsi di un livello, ma non andranno mai oltre il formaggio, che occupa 75 metri quadrati» ${ }^{9}$.

La piramide aiuta dunque anche a scegliere i cibi meno impattanti all'interno di una stessa categoria, ad esempio tra le carni quella avicola ha un'impronta ecologica di 46 metri quadri, quella bovina di 105, ma anche i tagli della carne hanno un impatto diverso: per produrre un chi- lo di filetto si emettono $68 \mathrm{~kg}$ di CO2; per un chilo di bistecca "solo" 42.

La frutta è il cibo più ecologico anche rispetto alla verdura, perché in media si usano meno fitofarmaci e concimi e la raccolta non elimina l'intera pianta, anche se consumare frutta fuori stagione inquina tre volte tanto rispetto alle disponibilità del momento.

Chi vive attorno al Mediterraneo, magari nelle regioni in cui è stata scritta la Bibbia, ha una dieta in genere migliore rispetto ad altre culture. La dieta mediterranea inquina circa la metà di quella nordamericana.

9. Tiziana Moriconi, Buon appetito, e salverai la terra, «espresso.repubblica.it», 28 giugno 2010 . 

I.2. II contributo delle religioni

aA 

Questi è dei nostri, oppure è straniero: così considerano $i$ pusillanimi;

ma per quelli dalle nobili gesta la terra è una sola famiglia

(Vidyākara, Subhāṣitaratnakośa

37,29; Mahāsubhāṣitasañgraha

2644; con varianti minori Val-

labhadeva, Subhāsitāvali 498)

Tutto questo mondo invero è il corpo di Viṣnu

(Viṣnupurāṇa 1,22,38)

Non sarebbe forse errato intravedere nella ideologia corrente della primazia del mercato una concezione propriamente religiosa, che divinizza il mercato stesso, considerato alla stregua di un attore interamente libero, in grado non solo di influenzare tra altri fattori, ma di decidere in modo assoluto, l'andamento dell'economia. Generalmente questa visione viene attribuita a un non meglio precisato Occidente, laddove a un altrettanto non meglio precisato Oriente viene attribuita una sensibilità ambientale di tipo ecologico ante litteram, anche nelle sue connotazioni propriamente religiose. A questo stereotipo non sfugge ovviamente l'hinduismo ${ }^{1}$.

Per quanto sia salutare diffidare dei luoghi comuni, non sarà inutile provare a verificare se effettivamente una qualche forma di sensibilità ambientale non sia rintracciabile nel

1. Il saggio che ha, in modo consapevole e determinato, posto l'accento sull'artificiosità della contrapposizione tra Oriente e Occidente e sulle colpe accademiche e politiche dell'orientalismo, è certamente il celebre pamphlet di Edward W. Said, Orientalism, Vintage Books, New York 1979. Per una radicale decostruzione dei presupposti ideologici e una altrettanto radicale critica della metodologia scientifica e dei supposti risultati di Said si veda almeno Robert Irwin, Dangerous knowledge: orientalism and its discontents, Overlook Press, Woodstock / NY 2006. 
sistema di valori dell'hinduismo. Se si getta lo sguardo sulla realtà contemporanea indiana non si può non rimanere colpiti dall'interesse suscitato dalle tematiche ambientali in molti movimenti talora anche di massa, spesso con una forte connotazione intellettuale e radicati sul territorio ${ }^{2}$.

Tra le iniziative maggiormente significative possiamo citare le numerose marce ( $p \bar{a}$ dayātrā) di ispirazione gandhiana (con riferimento alla marcia del sale svoltasi dal 12 marzo al 05 aprile 1930, una marcia di oltre duecento miglia, ca. 320 km, a piedi da Ahmedabad a Dandi, nello stato del Gujarat, sull'Oceano Indiano, con lo scopo di raccogliere una manciata di sale dalle saline, per rivendicare simbolicamente il possesso di questa risorsa preziosa al popolo indiano e l'ingiustizia della tassa di monopolio) in difesa di vari obiettivi di interesse ecologico ${ }^{3}$; il movimento (chipko andolan) che utilizza il gesto simbolico di abbracciare gli alberi per proteggere le foreste, fondato da Sunderlal Bahuguna il 26 marzo 1974, e successivamente per contrastare la costruzione della contestata diga di Tehri; l'iniziativa di Murlidhar Devidas Amte, noto come Baba Amte (Hinganghat, 26 dicembre 1914 - Anandwan, 9 febbraio 2008), avvocato e attivista indiano, vincitore del Templeton Prize for Progess in Religion del 1990, contro la costruzione della diga sulla Narmadā (campagna scaturita come reazione al disastro ambientale della Union Carbide a Bhopal nel 1984); l'adesione di numerosi templi, come quello di Śrī Ven̉kateśvara a Tirupati, a campagne di riforestazione (vanābhivṛdhi);

2. Si vedano N. P. Peritore, Environmental Attitudes of Indian Elites: Challenging Western Postmodernist Models, «Asian Survey» XXXIII (1993), pp. 804-818; C. K. Chapple, Nonviolence to Animals Earth and Self in Asian Traditions, State University of New York Press, Albany / N.Y. 1993; H. Coward (a cura di), Population Consumption and the Environment, State University of New York Press, Albany / N.Y. 1995; M. Gadgil, R. Guha, This Fissured Land: An Ecological History of India, University of California Press, Berkeley 1993; M. Gadgil, R. Guha, The Unquiet Woods: Ecological Change and Peasant Resistance in the Himala$y a$, University of California Press, Berkeley 1995; V. Narayanan, "One Tree is Equal to Ten Sons": Some Hindu Responses to the Problems of Ecology, Population and Consumption, "Journal of the American Academy of Religion», LXV (1997), pp. 291-332; V. Shiva, Staying Alive: Women Ecology and Development, Zed Books, London 1988; V. Shiva, The Violence of the Green Revolution, Research Foundation for Science and the Environment, Dehra Dun 1989; L. E. Nelson (a cura di), Purifying the Earthly Body of God, Religion and Ecology in Hindu India, State University of New York Press, Albany / N.Y. 1998.

3. M. Gadgil, The Unquiet Woods cit., pp. $101 \mathrm{sgg}$. 
Elementi di sviluppo sostenibile nell'hinduismo

campagne che si oppongono alla costruzione di mattatoi moderni intesi per l'esportazione delle carni macellate.

Va detto che in casi come l'ultimo citato, e ancora più nelle campagne per la salvaguardia della vacca, alcuni movimenti ecologisti hanno un'impronta politica decisamente ambigua e socialmente pericolosa, dal momento che si appoggiano alla destra fondamentalista hindu per perseguire i propri obiettivi con metodi talora pesantemente violenti, come l'aggressione (fino alla mutilazione o all'omicidio) nei confronti dei macellai o di coloro che trasportano carni macellate, spesso persone di religione islamica, per le quali il divieto di uccisione dei bovini non è in vigore. In questo quadro rientra l'uccisione di un ispettore di polizia nel distretto di Bulandshahr (Uttar Pradeś), avvenuta il 03 dicembre 2018. La vittima si chiamava Subodh Kumar Singh e aveva indagato sui macellai musulmani linciati in nome delle «vacche sacre». «L'ispettore era odiato dalle forze settarie», secondo Asad Hayat, avvocato della famiglia di Mohammad Akhlaq, ucciso nel 2015 a bastonate dai «vigilanti delle vacche» per il sospetto di aver consumato carne bovina. L'Uttar Pradeś è tra gli Stati indiani che hanno vietato il commercio e consumo della carne e imposto la chiusura dei mattatoi. Il bando è stato contestato da più parti, perché mette a rischio la sopravvivenza dei poveri, soprattutto musulmani e cristiani, che ne lavorano le pelli.

Un dialogo interdisciplinare fecondo tra ambientalisti e politologi ha dato luogo a una produzione che potremmo chiamare apologetica, che cerca di rintracciare (talora anche al di là della stretta verosimiglianza) nel corpus vedico le radici di un ambientalismo contemporaneo specificamente indiano. Ricordiamo in particolare due volumi, il primo a opera di due politologi, il secondo scritto da un'oceanografa ambientalista che vive in India ${ }^{4}$. In ambito strettamente indologico è rilevante l'impresa dovuta a Kapila Vatsyayan, che in un'opera collettanea in cinque volumi ne ha dedicato uno in particolare alla problematica ecologica in relazione con l'antropologia culturale, la cosmologia e la mitologia indiana ${ }^{5}$.

4. O. P. Dwivedi, B.N. Tiwari, Environmental Crisis and Hindu Religion, Gitanjali Publishing House, New Delhi 1987; M. Vannucci, Ecological Readings in the Veda, D.K. Print World, New Delhi 1994.

5. K. Vatsyayan (a cura di), Prakrti, the Integral Vision, New Delhi, Indira Gandhi Natio- 
Se volessimo rintracciare almeno alcuni dei riferimenti di carattere ecologico ante litteram nella letteratura upanisadica non dovremmo trascurare passi come Brhadāranyakopanișad 1,4,16, in cui nel contesto di uno dei primi riferimenti alla pratica dei «cinque sacrifici» (pañcayajña) si allude alla profonda solidarietà esistente tra i diversi livelli dell'universo, abitati rispettivamente da déi, veggenti, antenati, uomini e animali; $2,5,1$, in cui si fa riferimento all'interdipendenza tra la terra e gli esseri viventi. Potremmo moltiplicare i riferimenti. Ma forse sono due dei «grandi detti» (mahāvākya) su cui si basa la dottrina della scuola del vedānta che sono suscettibili di gettare luce sulla sensibilità ecologica dei maestri upanisadici. Entrambi provengono dalla Chāndogyopanișad: sarvam khalv idam brahma «tutto ciò invero è l'assoluto [brahman]» $(3,14,1)$ e tat tvam asi «tu sei quello» $(6,8,7)$. Secondo un interprete particolarmente autorevole e acuto «l'insegnamento definitivo dell'Advaita [non dualismo] è la realtà del Brahman. Lunità qui insegnata include senz'altro sia l'uomo sia la natura; ma dal momento che il primo e principale interesse dell'uomo è l'uomo stesso, non la natura, la verità incarnata in «tu sei quello», ossia la fondamentale identità del singolo e dell'assoluto, assume preminenza nell'insegnamento. Pertanto il discepolo deve concentrare la propria attenzione su tale aspetto della dottrina, e trascurare l'altro [aspetto], quello che si riferisce alla natura, in quanto più o meno secondario» ${ }^{6}$.

Lispirazione gandhiana delle politiche per la conservazione della vacca è motivo di comprensibile disagio per la destra fondamentalista hindu, perché nel padre fondatore dell'India contemporanea tale pratica era radicata nell'irrinunciabile adesione alla nonviolenza $(\text { ahims } s \bar{a})^{7}$, che ovviamente costituisce fonte di imbarazzo per i mestatori populisti. La bibliografia sugli aspetti ecologici del pensiero gandhiano (da considerare una specie di compagno di

nal Centre for the Arts 1995, 5 voll., in particolare il volume V. B. Saraswati (a cura di), Man in Nature, Indira Gandhi National Centre for the Arts, New Delhi 1995.

6. M. Hiriyanna, The Essentials of Indian Philosophy, Allen \& Unwin, London 1949, pp. 171 sgg. (parentesi quadre e corsivi nostri).

7. Sulle tematiche relative alla nonviolenza nel pensiero indiano si veda J. E. M. Houben, K. R. Van Kooij (a cura di), Violence Denied, Violence, Non-Violence and the Rationalization of Violence in South Asian Cultural History, Brill, Leiden 1999. 
Elementi di sviluppo sostenibile nell'hinduismo

strada dell'hinduismo) è vasta e ramificata, bastino pochi riferimenti ${ }^{8}$.

Infine si può accennare al tentativo di prendere in considerazione alcuni casi concreti di pratiche ambientali studiate all'interno di un movimento ecologista diffuso anche fuori dall'India e di due comunità marginali indiane particolarmente significative dal punto di vista antropologico: il movimento swadhyaya, la comunità dei bishnoi, la comunità dei bhil ${ }^{9}$.

Il movimento swadhyaya è un nuovo movimento religioso sorto a metà del xx secolo in alcuni stati occidentali dell'India, che ha evidenti ricadute ambientali, ma non è considerato dai suoi sostenitori un movimento ambientalista $^{10}$. Fondato da Pandurang Shastri Athavale (1920-2003) a partire dal 1942, prende nome dalla pratica dello studio personale (sanscrito suādhyāya, lett. «recitazione per sé, lettura individuale»), come strumento di introspezione a scopo spirituale, con esplicito richiamo al pensiero dei Veda, delle upanișad, e della Bhagavadgìtā, reinterpretato e adattato in chiave contemporanea. Dal momento che il principio divino risiede in ciascuno, le divisioni di classe, casta, appartenenza religiosa vanno tutte superate, miran-

8. M. P. Mathai, Ecology and Lifestyle: A Gandhian Perspective, Original paper presented at a National Consultation on "Global Ecology and the Indian Context", jointly organised by Orthodox Theological Seminary, Kottayam \& ISPCK, Delhi on July 2 \& 3, 2010 at Orthodox Theological Seminary, reperibile al link https://www.mkgandhi.org/articles/ ecology.htm (ultima consultazione 23/04/2019); John S. Moolakkattu, Gandhi as a Human Ecologist, «Journal of Human Ecology», XXIX (2010), n. 3, pp. 151-158; T. Weber, Gandhi And Deep Ecology, «Journal of Peace Research», XXXVI (1999), n. 3, reperibile al link https://www.mkgandhi.org/environment/envt.htm (ultima consultazione 23/04/2019).

9. Per una presentazione riassuntiva si veda P. Jain, Dharma and Ecology of Hindu Communities, Sustenance and Sustainability, Ashgate; Farnham, Surrey, England; Burlington, VT 2011.

10. Si vedano R. K. Srivastava, Vital Connections: Self, Society, God. Perspectives on Swadhyaya, Weatherhill Publications, New York, 1998; G. A. James (a cura di), Ethical Perspectives on Environmental Issues in India, APH Publishing Corporation, New Delhi 1999; J. T. Little, Video Vachana, Swadhyaya and Sacred Tapes, in L. A. Babb, S. S. Wadley (a cura di), Media and the Transformation of Religion in South Asia, University of Pennsylvania Press, Philadelphia, 1995, pp. 254-281; M. Paranjape, Dharma and Development: The Future of Survival, Samvad India Foundation, Delhi 2005; T.S. Rukmani, Turmoil, Hope, and the Swadhyaya, CASA Conference, Montreal 1999; B. M. Unterberger, R. Sharma Rekha, Shri Pandurang Vaijnath Athavale Shastri and the Swadhyaya Movement in India, "Journal of Third World Studies», Vol. 7, No. 1, Spring 1990, pp. 116-132; A. K. Giri, Self-Development and Social Transformations? The Vision and Practice of the Self-Study Mobilization of Swadhyaya, Lexington Books, Lanham 2009. 
do a sviluppare un'idea di fraternità degli esseri umani in dipendenza dalla comune paternità divina. Le risorse naturali, quali terra, acqua, alberi e bestiame, vanno accudite sulla base di tale principio. Questo aspetto ambientalista è forte nella componente indiana e rurale del movimento, meno in quella diasporica e urbana. Per quanto concerne la diaspora, il movimento è diffuso al di fuori dell'India negli USA, nel Regno Unito, nel Medio Oriente, in Africa, Australia, Nuova Zelanda, Suriname, isole Fiji, Indie occidentali, ovvero in zone in cui è presente una più o meno significativa diaspora indiana (siano i suoi membri ufficialmente rubricati come NRI, Non Resident Indian, o meno). Il movimento tende a mantenere una certa distanza da altre correnti hindu e non gradisce essere etichettato in questo modo. Fra i progetti di àmbito socio-economico sostenuti dal movimento si possono ricordare pratiche di coltivazione e di pesca (rispettivamente yogeśvara krși e matsyagandhā), definite esperimenti (prayoga) di tipo sociale. Figlio di un insegnante impegnato nella diffusione della Bhagavadgìtā secondo metodi tradizionali, quando suo padre cadde malato nel 1942, Athavale si dedicò alla prosecuzione della missione paterna, sino a quando nel 1954 in occasione della partecipazione a un convegno in Giappone raccolse la sfida di dimostrare che in India esistevano villaggi che si sostenevano sulla base della dottrina della Bhagavadgìtā. Sulla base di questo stimolo fondò nel 1956 una scuola residenziale (tattvajūāna vidyāpitha), che aveva come scopo lo studio e l'approfondimento del pensiero filosofico di stampo sacerdotale. La dottrina della presenza immanente del divino nell'uomo, individuata come la base speculativa di tutto l'edificio, lo portò a considerare l'autostima (asmitā, letteralmente «io-sono-tà») come la virtù fondamentale per mettere in pratica gli aspetti teologici e filosofici dell'insegnamento, il modo migliore di porre in pratica l'intuizione dell'identità dell'ātman, il principio cosciente individuale e del brahman, la forza fondante dell'universo, che senza un riscontro pratico sarebbe rimasta lettera morta. Si trattava insomma di rendere fruibile il messaggio della Gìtā alle masse, in primo luogo rurali, trasformando la teoria in pratica sociale, economica, ambientale. Tra le diverse strategie di contatto con le popolazioni locali si possono citare per esempio le visite in area suburbana (visite emozionali di due ore, visite de- 
Elementi di sviluppo sostenibile nell'hinduismo

vozionali di due giorni, rispettivamente bhäva pherī e bhakti pherī), i pellegrinaggi (tīrtha yātrā) nel corso dei quali gli attivisti invitano i vicini a un raduno religioso. Quest'ultima pratica ha avuto applicazione internazionale, quando attivisti dagli USA hanno lanciato campagne di pellegrinaggio in paesi come Suriname, Fiji, Trinidad, Australia e Nuova Zelanda. Gli incontri con la popolazione hanno lo scopo di radicare nei cittadini l'autostima, il senso della propria dignità personale, utilizzando in modo creativo anche altri aspetti dell'ortoprassi religiosa tradizionale come il culto dedicato all'undicesimo giorno del mese lunare, il digiuno rituale, il sacrificio del fuoco (ekādaśî, upavāsa, yajña). Dopo il 1970, sulla base di un passo della Gĩtã che esalta l'operosità ${ }^{11}$, il movimento ha promosso una campagna per convincere migliaia di professionisti in diversi settori (agricoltori, pescatori, medici, insegnanti, mercanti) a offrire parte del loro tempo e del loro bagaglio professionale a favore della comunità, come Arjuna offriva i frutti del suo agire al Signore Krṣna, coniando lo slogan «la devozione è una forza sociale (bhakti ek sāmājik śakti hai)». Tra i progetti più significativi lanciati dal movimento si possono citare i centri culturali per bambini, per adolescenti e per donne (Bāla Sam skāra Kendra; Divine Brain Trust; Mahilā Kendra). La ragione principale della diffusione limitata delle iniziative del movimento, che non ha mai oltrepassato i confini del Mahārāṣțṛa e del Gujarāt, è la scelta di utilizzare le lingue locali (marăthis, gujarātì); il successo è molto maggiore in ambienti rurali anziché in aree urbane. Sono presenti tentativi di costruire una sorta di ecoteologia, soprattutto tramite la divinizzazione del mondo vegetale, attraverso slogan come «Vāsudeva [patronimico di Krṣna] è negli alberi» (vrkșa main vāsudeva) e «Dio è nelle piante» (paudhe main prabhu): per spiegare la presenza divina nelle piante si ricorre ad argomentazioni di sapore tradizionale che fanno uso di paretimologie, per es. «There is a divine power in trees which makes it possible for water and fertilizer to rise from the roots below and reach the top portion against the gravitational force. It is not just the result of Keśäkarșaṇa (capillary action)

11. Bhagavadgìtā 18,46: "Da lui procede l'operosità degli esseri, da lui tutto quest'universo è stato dispiegato; rendendogli culto con l'azione che gli compete, l'uomo ottiene la perfezione». 
but it is Keśavākarșaṇa (Krṣna's force)» ${ }^{12}$. Altre iniziative di sapore ambientalista sono per es. la trasformazione di aree rifiutate dai poveri in dono perché desertiche e sterili in oasi naturalistiche protette, rivitalizzate tramite piantumazione di specie vegetali adatte (Buddha Vrkșamandira, area di 42 acri, con 1200 alberi da frutta, 200 erbe legnose e 150 alberi di 30 specie diverse).

I bishnoi (o vishnoi, termine che significa «ventinove») sono una comunità rurale del Rājasthān stanziata nei pressi di Jodhpur, fondata da Jambheśvara Bhagavan, noto comunemente come Jambhaji (1451-1536). Secondo il mito eziologico, il 9 settembre 1730 soldati del mahārāja di Jodhpur, Abhay Singh, presero ad abbattere alberi di khejari (Prosopis cineraria) come legna da ardere. Una donna del villaggio bishnoi nelle vicinanze, Amrita Devi, si precipitò per impedire l'operazione, abbracciando un albero, imitata dai suoi familiari e dagli altri membri dalla comunità: 363 persone furono uccise prima che la notizia giungesse al re, che diede ordine di fermare il massacro. Il nome della comunità, $b i$ shnoi, fa riferimento alle ventinove regole che ne costituiscono la legge di fondazione; il nome alternativo vishnoi cerca di riconnetterli al culto di Viṣnu, per assonanza, e costituisce un esempio di sanscritizzazione, del tipo top down, ossia di cooptazione di una tradizione locale subalterna nel sistema di valori della classe sacerdotale dominante ${ }^{13}$. Insediati in una zona a forte rischio di desertificazione, i bishnoi hanno una lunga esperienza di tecniche tradizionali per contrastarla. Sono una comunità liminale anche dal punto di vista storico-religioso, dal momento che si situano nella zona di interscambio culturale e religioso tra hinduismo e islam: sono ipotizzabili legami con una branca dell'ismailismo sciita $(\text { satpanth })^{14}$. Quando Jambheśvara aveva 25 anni cominciò

12. Pankaj Jain, Dharma and Ecology cit., p. 32.

13. Sulla sanscritizzazione si veda M. N. Srinivas, Religion and society among the Coorgs of South India, Clarendon Press, Oxford 1952, p. 32. Si vedano inoltre Id., The cohesive role of sanskritization and other essays, Oxford University Press, Delhi-New York 1989, nonché K. K. Gangadharan, Sociology of Revivalism. A Study of Indianization, Sanskritization, and Golwalkarism, Kalamkar Prakashan, New Delhi 1970 e S. Lal, From Higher Caste to Lower Caste. The Processes of Asprashyeekaran and the Myth of Sanskritization, Rawat Publications, Jaipur 1997.

14. Si veda D. Sila Khan, Crossing the Threshold: Understanding Religious Identities in South Asia, I. B. Tauris, London 2004. 
Elementi di sviluppo sostenibile nell'hinduismo

una grave siccità destinata a protrarsi per un decennio: il fondatore spirituale della comunità dei bishnoi lasciò il villaggio natale alla morte dei genitori nel 1484 e prese dimora a Samarathal, una collina sabbiosa nei pressi del villaggio di Mukām nel distretto di Nokha nei pressi di Bikaner. Dopo molti anni di meditazione e pratiche ascetiche, all'età di 34 anni, ebbe una visione, in cui vide uomini che entravano in conflitto con la natura e distruggevano l'ambiente che li sostentava. Decise quindi di dedicarsi a un programma di riforme sociali, nella convinzione che fosse dovere del genere umano sostenere l'ambiente e non opporsi a esso, per preservare le proprie fonti di sostentamento: fondò quindi la comunità dei bishnoi l'ottavo giorno della quattordicina scura del mese lunare di kārttika dell'anno 1542 dell'era vikrama (ev 1485). Da queste poche notizie storiche appare evidente come i bishnoi siano (più o meno legittimamente) considerati precursori o ispiratori inconsapevoli del succitato movimento (chipko andolan) fondato da Sunderlal Bahuguna. Le 29 regole dei bishnoi includono 8 norme relative alla conservazione e protezione degli animali e delle piante, compreso il divieto di sterilizzare i tori, il mantenimento dei capri nei santuari, il divieto di uccidere i capi di bestiame, di tagliare alberi e l'obbligo di proteggere ogni forma di vita. La proibizione di indossare vesti di colore blu è dovuta al fatto che la tintura per ottenere tale sfumatura comporta la distruzione di arbusti ritenuti degni di salvaguardia. Altre 7 regole riguardano il comportamento sociale: obbligo di essere sinceri, contenti di ciò che si ha, puri, di evitare l'adulterio e simili. Non è consentito criticare gli altri e bisogna sopportare le critiche che ci colpiscono. Altre 10 regole riguardano l'igiene personale e la necessità di mantenersi in buona salute: obbligo di bere acqua filtrata, del bagno quotidiano, di mantenere buone condizioni igieniche, divieto del consumo di oppio, alcool, tabacco e altre sostanze stupefacenti (tranne che in occasioni di determinate festività, in cui si consumano bevande contenenti oppiacei; come pure è consentito il commercio di oppiacei con popolazioni vicine che non sono membri della comunità). La dieta è tendenzialmente vegetariana; le donne sono soggette a segregazione per un mese dopo il parto e per cinque giorni durante il periodo mestruale. Le ultime 4 regole contengono norme che disciplinano le pratiche spirituali: il perpetuo ramme- 
moramento dell'onnnipresenza di Dio, l'esecuzione dei riti quotidiani, l'osservanza del digiuno rituale e la custodia del fuoco comunitario (havan) a ogni luna nuova. Oltre alle 29 regole di base sono in vigore 120 norme minori specifiche definite «parola [sacra]» (śabda), alcune di interesse ambientale. In epoca contemporanea i bishnoi sono noti anzitutto per il contrasto attivo al bracconaggio.

La comunità dei bhil (è dubbio se siano da considerare o meno «aborigeni», ādivāsi, etimologicamente «abitanti originari», termine di controversa applicazione $)^{15}$ si dedica alla conservazione di boschi sacri (variamente noti come deora, malvan, deorai, rakhat bani, oran, deo ghät, mandir van, bāgh; noi diremmo aree protette), in cui sono inclusi santuari dedicati a divinità, nei distretti di Banswara e Dungarpur nel Rājasthān meridionale. Circa il $45 \%$ di queste aree hanno dimensioni comprese tra uno e tre acri; $14 \%$ copre un'area di 30 acri, in cui vivono più di mille specie arboree, incluse essenze come neem (Azadirachta indica), pipal (Ficus religiosa), khejadi (Prosopis cineraria). Dal punto di vista delle risorse idriche, $31 \%$ comprende uno stagno, $17 \%$ una cascata, 38 $\%$ un pozzo, spesso non adeguatamente soggetti a manutenzione; le specie animali comprendono 54 tipi di mammiferi e rettili e 27 tipi di uccelli.

Le poche note raccolte in questo contributo intendono fornire un quadro di riferimento essenziale, ma soprattutto suscitare interesse per le tematiche ambientalistiche dei movimenti religiosi attivi nel subcontinente indiano, e sottolineare la necessità di studi specifici di tipo scientifico, per sfrondare il sottobosco dalle malerbe delle fake news e degli pseudomiti e consentire la crescita di una informazione sana e libera da fraintendimenti spesso voluti, e da strumentalizzazioni più o meno inconsapevoli, degli argomenti affrontati.

15. Si vedano S. Dasgupta, Adivasi studies: From a historian's perspective, «History Compass» 16.10 (2018): e12486, https://doi.org/10.1111/hic3.12486; S. C. Dube, Tribal Heritage of India, Vikas Pub. House, New Delhi 1977-; K. S. Singh, Tribal movements in India, Manohar Publishers, New Delhi 1982-1983; Id., The tribal situation in India, Indian Institute of Advanced Study, Shimla, 2002; Id., Tribal society in India: an anthropo-historical perspective, Manohar Publishers, New Delhi 1985. 

nell'Ebraismo

Paolo S. Pozzi

L'Agenda Onu 2030 per lo Sviluppo Sostenibile, sottoscritta nel settembre 2015 da 193 paesi membri dell'Onu, è un programma d'azione relativo alla prosperità delle persone ed alla salute del pianeta ${ }^{1}$. L'avvio degli Obiettivi per lo Sviluppo Sostenibile è iniziato sin dal 2016, ed indica un programma, con 169 'target' o traguardi, da raggiungere idealmente nell'arco dei prossimi 15 anni; i paesi, infatti, si sono impegnati a raggiungere questi traguardi entro il 2030. Tra i 17 Obiettivi per lo Sviluppo Sostenibile, gli obiettivi 2, 12 e 15 dell'Agenda Onu 2030 prendono in considerazione:

Ob. 2: porre fine alla fame, raggiungere la sicurezza alimentare, migliorare la nutrizione e promuovere un'agricoltura sostenibile

Ob. 12: garantire modelli sostenibili di produzione e di consumo

Ob.15: proteggere, ripristinare e favorire un uso sostenibile dell'ecosistema terrestre 
Paolo S. Pozzi
Sono sicuramente obiettivi dal forte significato etico e morale, nella loro finalità, con evidenti ripercussioni, potenzialmente di carattere immediato, sulla gestione delle risorse del pianeta; laddove, perlomeno per l'Obiettivo 2, la sicurezza alimentare appare rappresentata da sufficienti produzioni di tipo vegetale e animale, destinati all'alimentazione umana, e sulla loro redistribuzione; con evidenti considerazioni sul come e quanto utilizzare queste risorse.

$\mathrm{Al}$ di là degli aspetti puramente tecnici su come svolgere determinate opere di produzione (vegetale, animale, energetica, ecc.), va considerato come ogni scelta tecnica sia inevitabilmente anche legata ad una percezione di tipo culturale e, se vogliamo, anche morale, che determinate culture possano aver generato nei confronti di quella stessa attività.

Per fare un esempio, magari banale, in epoca non lontana, in Paesi sicuramente occidentali e quindi a noi vicini culturalmente la produzione di una piantagione poteva avere un valore intrinseco decisamente superiore al valore intrinseco dello schiavo che se ne occupava; o viceversa il valore di uno schiavo in relazione ai compiti da svolgere.

Largomento che mi è stato chiesto di trattare è se esista un punto di vista Ebraico su ambiente, animali, sicurezza alimentare? L'Ebraismo si occupa / si riferisce in qualche modo al mondo animale, all'ambiente circostante, alla sicurezza alimentare del prossimo? Esiste un approccio etico Ebraico agli animali, all'ambiente, alle necessità basilari/alimentari del prossimo?

Già il solo argomento etiche e animali rappresenta un argomento spinoso: «teorie tradizionali di tipo cristiano, kantiano, cartesiano o aristotelico hanno sostenuto che gli esseri umani sono titolari di uno status morale, mentre gli animali no, poiché gli esseri umani possiedono razionalità linguaggio e capacità di agire moralmente a differenza degli animali» ${ }^{2}$ con ciò apparentemente sottraendo parte del Creato (il mondo animale) ad eventuali considerazioni altro che strettamente utilitaristiche. 
Gli animali furono creati il giorno 5 e 6 della creazione, prima dell'uomo: «l'uomo fu creato prima di shabbath (proprio al termine della Creazione); perché mai? diventasse arrogante, digli: il moscerino ti ha preceduto nella creazione» ${ }^{3}$.

All'uomo fu detto: «e dominerete sui pesci del mare e sugli uccelli nel cielo» ${ }^{4}$. Ma anche: «Dio prese l'uomo e lo pose nel giardino dell'Eden per lavorarlo e per custodirlo ${ }^{5}$ e gli disse: «Guarda le mie opere, quanto sono belle e meravigliose! Le ho fatte tutte per voi. State bene attenti a non spogliare e distruggere il mio mondo; perché, se lo farete, nessuno potrà porvi rimedio» ${ }^{6}$.

Già dal principio, dunque, l'uomo è posto avanti agli animali ed al creato: come dominatore degli uni; come preservatore dell'altro. Ciò non preclude, comunque, un utilizzo sia degli uni che dell'altro: infatti Abel era pastore, Cain era agricoltore.

All'uomo viene dato:

- sia il controllo sugli animali («e dominerete») e anche il loro utilizzo: latte, lavoro, pelle (dopo morti); l'uccisione

è praticata, a certe condizioni: infatti Abel compiva sacrifici usando animali;

- che l'utilizzo della terra: Cain, infatti, era agricoltore.

$\mathrm{Ma}$, ancora, non viene loro permesso di mangiare carne ${ }^{7}$ e ciò sino al diluvio universale. Nel Talmud ${ }^{8}$ è riportato: «Rav Jehudha, a nome di Rav: ad Adamo non fu permessa la carne».

Era questa una situazione ideale?

Cain pensò che il livello dell'uomo fosse come quello degli animali, e perciò portò vegetali in sacrificio ${ }^{9}$. Dio non aveva

3. Talmud Babilonese; Sanedrin, 38:a.

4. Bereshit - Genesi 1:28.

5. Bereshit - Genesi 2:15.

6. Qohelet Rabbà: 7: 13; circa VI-VIII secolo.

7. Bereshit - Genesi; 1:29; S. Izchaki (Rashi) (1040-1105), ivi; A. Ebn Ezra (1092-1167), ivi; M. Maimonide (Ramban) (1194-1270), ivi; C. Ben Nachman (Hazchoni) (12501310), ivi; O. Sforno (1470-1550), ivi.

8. Talmud Babilonese; Sanhedrin 59:b.

9. Y. Albo, Sefer HaYkarim (1380-1444), Spagna. 
richiesto alcun sacrificio ${ }^{10}$ (Cain ideò la cosa; Abel lo imitò, portò, come Cain, "ciò che aveva a portata di mano» ${ }^{11}$ ovvero pensò che gli animali si potessero offrire in sacrificio); questa situazione portò alla gelosia ${ }^{12} \mathrm{di}$ Cain; la gelosia all'omicidio. Dopo il diluvio universale, la situazione cambia radicalmente, ed ai discendenti di Noè viene dato il permesso esplicito a nutrirsi di animali: «ogni essere vivente sarà cibo per voi; come l'erba verde, Io vi ho dato tutto questo» ${ }^{13}$. «Poiché (gli animali) si salvarono nell'arca che avete costruito, e per mano vostra è arrivata la salvezza, ecco, essi sono nelle vostre mani affinché ne facciate ciò che è giusto ai vostri occhi» ${ }^{14}$.

\section{Lo status degli animali dopo la rivelazione della Torà}

Nella Torà sono presenti diversi comandamenti relativamente agli animali.

Un comandamento viene particolarmente considerato dai Maestri: «Quando vedrai l'asino del tuo nemico accasciato sotto il peso del suo carico e ti asterrai dall'aiutarlo, aiutalo insieme a lui» ${ }^{15}$.

«Disse Rabà: (da qui)... studiamo che il (divieto al) maltrattamento degli animali è un comandamento diretto dalla Torà» ${ }^{16}$.

Mentre Rashi commenta: «[aiutalo] a scaricarlo dal peso; a toglierli il peso di dosso» ${ }^{17}$.

Tu sei avvisato «a non rimanere inattivo di fronte alla sofferenza dell'animale, persino se il suo proprietario è un tuo nemico» ${ }^{18}$.

L'Ebraismo da qui deriva sia l'obbligo di aiutare o salvare un animale (comandamento fattivo/positico), che di astenersi dal compiere azioni lesive o dolorose sugli animali (comandamento astensivo/negativo).

10. A. Warshavsky, A. Lotan, R. Magidov, E. Paz, Alilot HaRashit Le Trame PrincipaliLomicidio di Abel, MTC Ed. Ramat Aviv, Israel 2003, p. 81.

11. O. Sforno (1470-1550) chiosa su Bereshit, Genesi 4:2.

12. O. Sforno (1470-1550) chiosa su Bereshit, Genesi 4:8.

13. Bereshit - Genesi 9:3.

14. C. Ben Nachman (Hazhoni) (1250-1301), ivi.

15. Shemot - Esodo 23:5.

16. Talmud Babilonese; Bavà Mezzia:32:b.

17. S. Izchaki (Rashi) (1040-1105), ivi.

18. C. Ben Nachman (Hazchoni) (1250-1310), ivi. 
Salvare un animale da ogni situazione dolorosa o pericolosa, minimizzare ogni evento traumatico sono considerati obblighi (mitzwa me-de-Oraita) della Torà ${ }^{19}$, così come esemplificato dall'asino sovraccarico.

Astenersi da ogni azione intenzionalmente traumatica, astenersi da ogni azione dolorosa gratuita sono considerati obblighi istituiti dai Maestri (mitzwa me-de-Rabbanan) ${ }^{20}$.

Peraltro, anche se oramai di diminuita importanza rispetto al passato, l'uso degli animali è permesso, ma molto regolato, con precisi obblighi alla proibizione di sfruttamento ed abusi.

A titolo di esempio si possono riportare:

Proibizione ad aggiogare insieme, allo stesso lavoro, asino e bue $^{21}$ : «Dio ha compassione per tutte le sue creature, e l'asino non ha la forza del bue» ${ }^{22}$.

Non mettere la museruola al bue che trebbia ${ }^{23}$ : «Nel momento in cui utilizziamo un animale per lavoro, dobbiamo riconoscerli un compenso» $[\ldots]$ «per insegnarci che la nostra anima sia un'anima bella, che sceglie la correttezza e vi si attacca; ed insegue gentilezza e compassione, e la abituiamo anche al fatto che gli animali non son stati creati solo per nostro uso» ${ }^{24}$.

Obbligo a foraggiare gli animali: «e nel tuo campo provvederò foraggio per il tuo bestiame e tu mangerai e ti sazierai ${ }^{25}$. «E vietato ad una persona di mangiare prima che abbia dato da mangiare ai suoi animali» ${ }^{26}$ (in quanto il riferimento al bestiame precede quello all'uomo).

Obbligo al riposo: «sei giorni farai i tuoi lavori, ma il settimo giorno dovrai smettere in modo che il tuo bue ed il tuo asino possano riposare; e si riposi il figlio della tua serva e lo straniero (presso di te)» ${ }^{27}$. $«$ Dagli tregua; permettigli

19. Y. Karo, Shulchan Aruch- Orach Haym, 1563, 305:19.

20. A. HaLevi, Sefer Ha Chinuch, circa xin secolo, ch. 451; Bahaba"d Y., Minchat Chinuch, circa 1800 , ch. 451 .

21. Devarim - Deuteronomio 22:10.

22. A. Ibn Ezra (1089 -1167), ivi.

23. Devarim - Deuteronomio 25:4.

24. A. HaLevi, Sefer cit., ch. 596.

25. Devarim - Deuteronomio 11:15.

26. Talmud Babilonese; Berachot, 40 :a.

27. Shemot - Esodo 23:12. 
che pascoli e mangi dal campo. O invece non è altro che confinarlo/imprigionarlo in casa? questo non è riposo, bensì crudeltà» 28 .

Obbligo al rifugio e restituzione di animali perduti: «Se ti imbatterai nel bue del tuo nemico o nel suo asino che si è smarrito, dovrai ricondurglielo» ${ }^{29}$; «non vedrai il bue o la pecora del tuo prossimo che si sono perduti e li ignorerai $[\ldots]$ condurrai in casa tua fino a che il tuo prossimo l'avrà richiesto e glielo restituirai [...] non potrai ignorare» ${ }^{30}$.

$\mathrm{Al}$ di là di implicazioni anche evidentemente utilitaristiche e sociali (tutela del "bene altrui"), non si possono ignorare alcune implicazioni animal welfare ante litteram, come nel commento di Rashi sull'interpretazione di riposo sabbatico non meramente interpretata come semplice astensione dal lavoro dell'animale.

Come già accennato, il permesso esplicito a nutrirsi di animali è dato solo dopo il diluvio: «ogni essere vivente sarà cibo per voi; come l'erba verde, Io vi ho dato tutto questo» ${ }^{31}$, ma con precise limitazioni le quali, riferendosi al tempo di Noè, ovvero antecedentemente ai Patriarchi ed al concetto stesso di popolo Ebraico o di Israele, hanno conseguentemente un valore intrinseco per l'intera umanità: «solamente non potrete mangiare la carne con la sua vita, che è il sangue» ${ }^{32}$.

Interessanti i commenti dei Maestri a proposito: l'espressione "sua vita» implica il divieto a cibarsi di un animale ancora in vita ${ }^{33}$; ovvero l'obbligo ad uccidere completamente un animale prima di nutrirsene. Ed in effetti: «non c'è cosa più crudele di tagliare della carne o un organo da un animale mentre è ancora in vita» ${ }^{34}$.

I diversi comandamenti e commenti che trattano del nutrirsi di animali evidenziano il concetto che, malgrado ci sia permesso nutrirci di animali, ciò deve essere fatto in modo

28. S. Izchaki (Rashi) (1040-1105), ivi.

29. Shemot - Esodo 23:4.

30. Devarim - Deuteronomio 22:1,2.

31. Bereshit - Genesi 9:3.

32. Bereshit - Genesi 9:4.

33. S. Izchachi (Rashi) (1040-1105), ivi.

34. A. HaLevi, Sefer cit., ch. 452. 
che la eventuale sofferenza indotta sia la minore possibile ${ }^{35}$; fermo restando che la sofferenza coscentemente indotta è comunque vietata dai Maestri.

Altri obblighi o comandamenti (mitzwot) della Torah, cosi come enunciati, non sembrano avere un senso strettamente legato all'evitare sofferenze fisiche dell'animale o a rispettarne le esigenze fisiologiche basilari (nutrizione, ecc.); e per questi appaiono particolarmente interessanti i commenti dei Maestri.

Proibizione di macellare animali molto giovani (entro gli otto giorni di vita): «e starà sette giorni sotto sua madre e dall'ottavo giorno in avanti sarà gradito come sacrificio» ${ }^{36}$; «appena nato, un animale è inadatto sia come regalo, sia per venderlo, sia per nutrirsene [...] è "incompleto" ${ }^{37}$.

Proibizione a raccogliere, in natura, uova o pulcini in presenza della madre: «se ti capiterà un nido d'uccello su un albero o per strada, pulcini o uova, e la madre cova sui pulcini o sulle uova, non prenderai i figli dalla madre; manderai via la madre e prenderai i figli; così che te ne derivi del bene e si allunghi la tua vita ${ }^{38}$. «Se la madre è lasciata libera e si allontana, non si addolora ${ }^{39}$; "per infondere nei nostri cuori che la provvidenza di Dio Benedetto è sulle sue creature come sull'uomo» ${ }^{40}$; "per non estinguere gli uccelli dei campi, che sono res nullius, e ciò attraverso l'allontanare la madre» ${ }^{41}$.

I quali commenti fanno pensare ad un pensiero "ambientalista" ante litteram.

Viene considerata una sofferenza, negli animali, non di tipo strettamente "fisico"?

La Torà vieta di macellare madre (genitore) e figlio insieme lo stesso giorno: «Il bovino o l'ovino, lui e suo figlio non macellerete lo stesso giorno» ${ }^{42}$ ed i maestri spiegano: «per

35. Y Karo, Shulchan Aruch-Orach Haym, 1563, 305:19; D. Einger, Sulla carne e sulla morale - maltrattamento degli animali ed Ebraismo, Istituto “Torà e Terra", 2015, in www.toraland.org.il.

36. Va-Ikra - Levitico 22:28.

37. A. HaLevi, Sefer cit., ch. 293.

38. Devarim - Deuteronomio 22:6.

39. M. Maimonide (Rambam) (1138-1204), More' Nevuchim, 3:48.

40. A. HaLevi, Sefer cit., ch. 545.

41. O. Sforno (1470, 1550), ivi.

42. Va-Ikra - Levitico 22:28. 
fissare nella nostra anima la compassione ed allontanare la crudeltà» ${ }^{43}$. "In effetti l'animale ne soffrirebbe moltissimo; e non c'è differenza tra la sofferenza dell'uomo e quella degli altri animali ... giacché l'amore e l'affetto della madre per il figlio non segue la ragione» ${ }^{44}$.

«Se la Torà vigila su questi dolori persino nel caso degli animali di terra e degli uccelli, che cosa farà nei confronti dell'uomo?» ${ }^{45}$.

Anche se Rambam ammette che secondo la Torah gli animali possano avere sensazioni e sentimenti, così come tutti gli esseri viventi, e non solo l'uomo, ciò nonostante, la ragione delle mitzwot sta nell'influenza negativa che l'indurre sofferenza può avere sull'uomo stesso, abituandolo al male.

Un capitolo a sé nell'Ebraismo è rappresentato dalla macellazione degli animali (permessi o adatti-kasher).

Se la Torà lo considerasse inadatto o crudele, non esisterebbe la shechità quale metodo di macellazione.

La shechità è stata indicata dalla Torah quale mezzo misericordioso per l'uccisione degli animali da consumarsi. Il testo biblico, in senso stretto, non prescrive o indica precisamente le modalità di macellazione consentita. Il versetto: «allora macellerai, nel modo in cui ti ho prescritto, dal tuo bestiame e dal tuo gregge che l'Eterno ti avrà dato, e ne mangerai entro le tue città a tuo piacimento» ${ }^{46}$ viene interpretato dai Maestri quale rimando alla tradizione orale - Torà shebealpè ovvero nel modo in cui ti ho prescritto oralmente; codificando quindi «sull' esofago e sulla trachea; $\mathrm{e}$ negli uccelli, anche sulle vene» ${ }^{47}$; ed «in un animale grosso, anche il taglio dei vasi» ${ }^{48}$; essendo il modo finalizzato ad indurre la morte dell'animale senza provocare inutili sofferenze: «ed ancora si può dire sulla shechità fatta sul collo e con un coltello controllato, allo scopo di non indurre inutile sofferenza all'animale ${ }^{49}$; considerazione animal welfare ante litteram quando riportata al xiı secolo.

43. A. HaLevi, Sefer cit., ch. 294.

44. M. Maimonide (Rambam) (1138-1204), More' Nevuchim, 3:48.

45. S. Izchachi (Rashi) (1040-1105), ivi.

46. Devarim - Deuteronomio 12:21.

47. Talmud Babilonese, Hullin, 28:a.

48. Rabenu Hananel, circa XI secolo, chiosa su Talmud Babilonese, Hullin, 28:b.

49. A. HaLevi, Sefer cit., ch. 451. 
Ritengo opportuno ricordare ancora la proibizione di castrare: «non farete di queste cose ${ }^{50}$, sicuramente osservata, perlomeno in Israele, relativamente agli animali d'allevamento; permessa da alcuni Maestri moderni sotto forma di castrazione temporanea (legamento dotti deferenti e/o tube; castrazione ormonale) per il controllo delle popolazioni selvatiche/reinselvatichite (controllo del randagismo) ed aventi come obiettivo un beneficio collettivo e/o la prevenzione di malattie.

Se da una parte, come accennato, l'utilizzo degli animali in campo lavorativo - e le norme connesse - trovano oramai scarsa o nulla applicazione, le stesse norme hanno trovato declinazioni in forme attuali relativamente alle condizioni di mantenimento in senso generale degli animali: obbligo all'alimentazione, compresi i randagi urbani, che, tollerati e diventati concittadini oramai dipendono dall'uomo; e per i quali la Corte Suprema di Israele ha proibito l'abbattimento quale risposta automatica all'eventuale "semplice fastidio" provocato dalla loro presenza $^{51}$; l'obbligo alla cura dalle malattie; l'assistenza al parto; le limitazioni nell'utilizzo per scopi medici; la proibizione, in senso lato, a pratiche crudeli. A titolo di esempio riporto il divieto all'immobilizzazione e/o alimentazione forzata delle oche destinate alla produzione di foie gras $^{52}$; il divieto ad assistere alle corride ${ }^{53}$; il divieto alla caccia quale «inutile e pericolosa distruzione di vite» ${ }^{54}$; il divieto all'allevamento del "vitello a carne bianca" e ciò in quanto "anche se si possa considerare che le necessità dell'uomo possano giustificare una certa sofferenza nell'animale, sicuramente l'allevamento del vitello a carne bianca va a beneficio solo di una piccola parte della popolazione; e ciò non giustifica quel tipo di sofferenza ${ }^{55}$; ed infatti in Israele le condizioni migliorative richieste per l'allevamento a carne bianca hanno di fatto indotto alla loro chiusura; il divieto della pelliccia ${ }^{56}$.

50. Va-Ikra - Levitico: 22:24.

51. Petizione alla Corte Suprema di Israele, Baga"z 4844/2000.

52. O. Yossef, Sheelot ve-teshuvot, 15/02/2012, http://www.halachayomit.co.il.

53. O. Yossef, Sheelot cit., 17/10/2010.

54. Y. Landau, Noda B'Yehudha, su “Yore' De'a”, 1755, domanda 10.

55. D. Einger, Sulla carne cit..

56. Y. Ariel, in D. Einger, Sulla carne cit. 
Paolo S. Pozzi

\section{Qual è il significato?}

La natura esiste ed ha le sue leggi; il compito dell'Ebreo è di essere misericordioso nei confronti della natura, malgrado le sue leggi ci appaiano a volte spietate.

I comandamenti mizwot sono dati non per cambiare l'ordine della creazione, ma per elevare l'uomo rispetto alla natura e per impregnare l'animo umano di qualcosa di divino, cioè la misericordia di Dio stesso relativamente al creato ed alle creature: «buono è Dio per tutti; e misericordioso per tutto il suo creato» ${ }^{57}$. Si racconta ${ }^{58}$ di un vitello, destinato al macello, che corse a nascondersi sotto il mantello di Rav Yehudah; ma questi lo cacciò, dicendogli: «va'! perché per questo sei stato creato». Non che ciò non fosse vero! ma la mancanza di pietà dimostrata, costò a Rav Yehudha un lungo periodo di sofferenze. Queste cessarono il giorno in cui impedì alla sua serva di buttar fuori di casa «una topolina con i suoi piccoli ${ }^{59}$.

La astensione dalla carne o dalle cose in genere, non è richiesta dalla Torà; d'altronde la Torà non richiede di mangiare carne tutti i giorni, bensì in momenti particolari quali, quando esisteva il Santuario a Gerusalemme, l'obbligo al sacrificio pasquale; al consumo dei sacrifici entro la mattina successiva, etc. D'altro canto i Maestri hanno compreso come fosse nella natura dell'uomo cercare soddisfazione, anche nel mangiare:

un uomo è obbligato a rallegrare i suoi figli e la sua famiglia durante le Feste; con cosa ci si rallegra? Rav Yehudha Ben Batira dice: quando esiste il Santuario, non c'è allegria se non nella carne; ed ora che non esiste il Santuario, non c'è allegria se non nel vino. ${ }^{60}$

Una eventuale estensione o diminuzione dal consumo di carne sono considerati più da un punto di vista di salute dell'uomo ed ambientale, ma non come ideale morale ${ }^{61}$. Ciò anche se pensatori determinanti dell'Ebraismo moderno e sionista individuino in un ideale di «vegetarianesimo e

57. Salmi: 145:9.

58. Talmud Babilonese, Bava Mezia, 85:b.

59. A. HaLevi, Sefer cit., ch. 451.

60. Talmud Babilone, Pessachim, 109:a.

61. D. Einger, Sulla carne cit. 
pace» un riavvicinamento a quel mondo ideale rappresentato dall'esperienza dell'Uomo all'inizio della Creazione ${ }^{62}$, ovvero prima del consumo di carne da parte dell'umanità.

La Torà non vieta l'uccisione degli animali per utilizzarli quale alimento.

La Torà insegna la santificazione attraverso l'utilizzo di esseri viventi e cose: «e mangerai, ti sazierai e benedirai» ${ }^{63}$.

La Torà, viceversa, vieta lo spreco del creato: esseri viventi e cose; vieta la distruzione della creazione.

È forse questo il punto di contatto con la seconda parte del tema assegnatomi: Ebraismo ed ambiente

Come già ricordato: «il Signore prese l'uomo e lo pose nel giardino dell'Eden perché lo lavorasse e lo custodisse ${ }^{64}$ ovvero: «lo irrigasse e lo difendesse dagli animali, che non entrassero e lo sporcassero ${ }^{65}$.

Nel Pentateuco, laddove si vieta di tagliare alberi da frutta per utilizzarli durante l'assedio di una città ${ }^{66}$, i Maestri derivano il concetto di non distruggerai. Questo comandamento viene esteso dai Maestri al divieto a distruggere in senso lato: cose, animali, cibo; e viene riportato diverse volte nel Talmud; "per insegnare alla nostra anima ad amare ciò che è buono ed utile ed attaccarsi ad esso» ${ }^{67}$. Da qui si deriva il divieto a distruggere inutilmente le cose: bruciare, rompere un oggetto per nulla, strappare un vestito, ecc.

Il divieto al danno è a priori; anche se si ha intenzione o possibilità di ripagarlo economicamente ${ }^{68}$ : «e non solo relativamente agli alberi, ma persino se qualcuno rompe degli utensili/vasellame, o strappa vestiti o butta giù una casa, o occlude una fonte d'acqua o spreca del cibo, viola il "non distruggere" 69.

Permesso invece l'utilizzo, l'abbattimento per evitare danni, o di alberi molto vecchi, o oramai improduttivi.

62. D. Cohen (HaNazir), Visione sul vegetarianesimo e sulla pace - I rapporti tra l'uomo e gli animali ai giorni nostri e nei giorni a venire, in "Peles", Rabinovitz Ed., Poltava (Ukraina), circa 1904 (trascritti da lezioni e scritti di A.Y. Rav Kook, di cui era allievo).

63. Devarim - Deuteronomio, 8:10.

64. Bereshit - Genesi 2:15.

65. A. Ebn Ezra (1092-1167), ivi.

66. Devarim - Deuteronomio, 20:19-20.

67. A. HaLevi, Sefer cit., ch. 429.

68. M. Maimonide (Rambam) (1138-1204), Mishne Torah, Nizkei Mamon, 5:1.

69. M. Maimonide (Rambam) (1138-1204), Mishne Torah, Melachim-Milchamot, 6:10. 
Rav M. Gerstenfeld attribuisce a questi insegnamenti almeno due significati: rispettare i fondamenti della natura; conservare le risorse naturali che costituiscono il nutrimento per l'uomo, ottenuto solo dopo molto tempo e lavoro, prestando attenzione alle conseguenze di come si utilizzano le risorse naturali ${ }^{70}$.

Anche il consumismo è considerato dai Maestri una forma di "inutile distruzione" delle cose; anche se ovviamente il concetto si è evoluto con l'evolversi dei tempi.

Rav Zutra' paragonava «l'uso eccessivo di olio per la lampada» al non distruggerai ${ }^{71}$. Secondo Rambam «chiunque metta molti vestiti ad un morto, viola non distruggerai» ${ }^{72}$.

Secondo Rav S.R.Hirsh: «il divieto di distruggere si estende anche agli oggetti di proprietà della persona stessa [...] il divieto è un avvertimento globale, a che l'uomo non sfrutti il suo status nel mondo per distruggere senza ragione [...] l'uomo deve fare del mondo un uso oculato e saggio» ${ }^{73}$. Molti esempi ed insegnamenti ci vengono da shu"t (sheelot ve-theshuvot - domande e risposte, rivolte ai Maestri di tutti i tempi ed accuratamente trascritte) anche moderni.

Sul distruggere prodotti agricoli per allineare i prezzi, Rav H.D.Levi (Rav HaRashi, Tel Aviv, 1976) stabiliva: «meglio distribuire ai poveri che non comprerebbero comunque; solo se non c'è interesse, è possibile eliminare in modo onorevole»

Relativamente alla cura dell'ambiente che ci circonda, vorrei almeno citare la prima e maggiore delle preoccupazioni del mondo civilizzato: lo sgombero dei reflui fognari al di fuori dei centri abitati, problema che in alcuni Stati, o sub-continenti, si trascina ancora oggi ${ }^{74}$. Problema questo, ben evidenziato e regolato già ai tempi dell'esodo del popolo Ebraico: «dovrai avere uno spazio dedicato ai tuoi bisogni fuori dell'accampamento; [...] scaverai una buca per

70. M. Gerstenfeld, The environment in the Jewish Tradition: a sustainable world, Inst. for Israel Studies Edit., 2002.

71. Talmud Babilonese, Shabat, 7:b.

72. M. Maimonide (Rambam) (1138-1204), Avelut, 14:24.

73. S.R. Hirsh (1808-1888), Horeb: A Philosophy of Jewish Laws and Observances, Judaica Press, 2002, ch. 56.

74. India Government; Open Defecation Free (ODF) project; https://swachhbharat. mygov.in/. 
coprire le tue feci» ${ }^{75}$; «poiché tutto si rivela agli occhi, e ciò è odioso; genera avversione nell'animo» ${ }^{76}$; $"$ e l'animo si rende odioso attraverso l'abitudine all'odioso» ${ }^{77}$. Alle preoccupazioni cogenti si affianca però anche la visione di una "città ideale", circondata da campi «per essere di decorazione alla città, e non sia permesso di costruirvi case, piantare vigne, seminare» ${ }^{78}$.

I già citati Obiettivi 2 e 15 dell'Agenda Onu hanno come programma, rispettivamente: raggiungere la sicurezza alimentare; proteggere, ripristinare e favorire un uso sostenibile dell'ecosistema terrestre.

Nell'antico Israele l'utilizzo della terra ai fini agricoli era rigorosamente regolamentato: decisamente non tutto il prodotto dei campi era ad esclusivo utilizzo o godimento economico del proprietario del campo. Durante la mietitura/raccolta, un "angolo del campo", senza misura predefinita nella Torah; ma ridefinita ad almeno 1/60 del raccolto dai Maestri, doveva essere lasciato intonso ${ }^{79}$ e destinato ai poveri del luogo.

Altra misura/mitzwah di carattere sociale era costituita dal divieto alla spigolatura, ovvero il tornare indietro nel campo o nella vigna a raccogliere ciò che ci era caduto o sfuggito $^{80}$.

"Doni e decime" costituivano, invece, porzioni del raccolto da destinare ai Sacerdoti, che non possedevano terra, ed anche, a seconda degli anni calcolati su un ciclo settennale, ai Leviti e/o nuovamente ai poveri del luogo. Tali porzioni di raccolto e produzioni agricole, essendo predestinate a soggetti ben individuati, non potevano essere immesse sul libero mercato e, in una società fortemente o principalmente agricola, venivano gestite/distribuite in modo letterale (libero accesso ai campi ed ai raccolti; vedi oltre nel rimando all'episodio di Ruth). Il principio di non

75. Devarim - Deuteronomio 23:13-14.

76. A. Ebn Ezra (1092-1167), ivi.

77. Torah Temima, Ed. Mossad HaRav Kook, Jerusalem, 1993, chiosa 56 ivi.

78. M. Ben Nachan (Ramban-Nachmanide) (1194-1270), chiosa su BaMidbar-Numeri, $35: 2-5$.

79. VaIkra-Levitico, 19:9; VaIkra-Levitico, 23:22.

80. A. HaLevi, Sefer cit., ch. 219; M. Maimonide (Rambam) (1138-1204), Mishne Torah, Nizkei Mamon, 5:1. 
Paolo S. Pozzi immissibilità sul libero mercato (Israeliano) vige tuttora, ma gestito sottoforma di "scorporo" (su cui tornerò a breve) e quantificazione economica di queste porzioni di produzione dedicate/predestinate ${ }^{81}$.

Ma l'esempio forse più conosciuto al mondo relativo all'utilizzo della terra è costituito dall'istituzione dell'Anno Sabbatico o Shemita. La Shemita costituisce una mitzwah - comandamento della Torà e, relativamente alla gestione dei terreni, è menzionata in Va-Ikra - Levitico, 25: 3-6; in Shemot - Esodo, 23:10-11. In realtà l'Anno Sabbatico si compone di una serie di 10 mitzwot - comandamenti specifici, di cui 6 legati alla gestione della terra e 4 legati al "sabbatico del denaro" ovvero alle forme di remissione dei debiti.

Va sottolineato che la Shemita è esclusivamente relativa alla terra di Israele e nella sua estensione Biblica e non politica/statuale di oggi, con precise implicazioni sulla accettabilità di questi prodotti da parte della clientela Ebraica, Israeliana e non.

Spiego meglio: nella tradizione Ebraica, sinteticamente spiegando, i cibi vengono suddivisi in permessi - kasher - o proibiti, cosiddetti taref. Mentre nel resto del mondo, ed agli occhi della popolazione non Ebraica, il concetto di "proibito per gli Ebrei”, si associa prevalentemente ad alcuni tipi di carne (maiale; cavallo; coniglio) o di acquatici (crostacei; molluschi), nella terra di Israele, sia allora che oggi, il proibito si applica anche alle produzioni agricole e derivati.

Pur non esistendo frutti o verdure o raccolti proibiti di per sé, questi diventano proibiti se "angolo", "doni” e "decime" non sono stati scorporati dal raccolto. Ciò fermo restando il divieto all'effettuare la spigolatura di campi e vigne, cosa questa che, se evidenziata, non permetterebbe di produrre kasher a prescindere dallo scorporo successivo di "angolo", "doni" e "decime". In una società prevalentemente urbanizzata ed un'agricoltura fortemente meccanizzata, lo scorporo viene calcolato ed effettuato in denaro da un "tesoro del Rabbinato" ed utilizzato per i meno abbienti, attraverso zedakot - giustizie -, grosso modo equivalenti alle caritas, ritornando quindi all'obiettivo principale. In assenza 
di questo scorporo certificato, la produzione specifica (uve e vino; cereali e derivati: farine, pane, prodotti da forno; frutta, verdure) non ottiene la certificazione di kasher e come tale non entra nel circuito della GDO per la popolazione Ebraica, che in Israele costituisce circa lo 82-85\%.

Mutatis mutandis, il concetto si applica anche alle produzioni spontanee durante la Shemita ${ }^{82}$ : mentre relativamente alla gestione della terra, sono proibiti in modo assoluto aratura, seminatura, falciatura, potatura degli alberi e delle vigne; il raccolto di frutta e di ciò che è spontaneamente cresciuto, è permesso in particolari condizioni. Le condizioni sono, sostanzialmente, che i prodotti siano res nullius, ovvero a disposizione di tutti.

Allo scopo di difendere i poveri, la Torà ci comanda molte zedakot - atti di giustizia -: dare la zedak $\grave{a}^{83}$ - l'elemosina -, la decima del povero, la spigolatura, la dimenticanza (nel campo), l'angolo, ecc., ed anche il comandamento della Shemita. In questo anno i prodotti (della terra) saranno res nullius, in modo che i poveri possano goderne. ${ }^{84}$

Nuovamente, in un mondo di agricoltura moderna, meccanizzata e, soprattutto, urbanizzata, di fatto non si assiste al libero girovagare per i campi da parte dei meno abbienti. Ciò non inficia minimamente le mitzwot - comandamenti relativi -, che trovano una forma alternativa di applicazione. Fermo restando che la violazione al divieto di arare, seminare, falciare, potare, durante la Shemita squalifica totalmente i prodotti rendendoli non kasher e quindi non distribuiti dalla GDO rivolta al pubblico Ebraico, maggioranza in Israele, la produzione spontanea, prevalentemente frutta e tipi particolari di verdure, durante la Shemita viene raccolta attraverso un meccanismo che la affida ad un "tesoro del Rabbinato", già citato, e la riversa sul mercato al solo costo di raccolta/manodopera, ebraicamente permesso; quindi a prezzi di mercato inferiori e più convenienti, e con appo-

82. Sono presenti anche altre soluzioni, compatibili con la legislazione religiosa ebraica; ho preferito portare ad esempio quella con più evidenti implicazioni sociali, ed apparentemente anche maggiormente preferita dai consumatori.

83. Zedakà deriva dalla radice ebraica zdk: zodek, zedek: giusto, giustizia; la cosiddetta elemosina è in verità un gesto di giustizia nei confronti di chi ha bisogno.

84. Y.Z. Rimon, Shemita - halacha (legislazione ebraica) dalla fonte, Maggid, in maggid@ korenpub.com. 
Paolo S. Pozzi sita "certificazione" in etichetta sulla procedura eseguita. In assenza di tali certificazioni, i prodotti della Shemita, sia durante l'anno della Shemita che in seguito, non hanno mercato quali prodotti kasher, con le inevitabili conseguenze in un Paese a maggioranza Ebraica, in cui il 76-80\% degli Ebrei dichiara di mangiare kasher $^{85}$. Accettabili forme alternative per piccolissimi produttori o produzioni famigliari, sono la raccolta giornaliera per il proprio fabbisogno, non superiore all'equivalente di «tre pasti per famiglia» ${ }^{86}$ e l'esposizione gratuita al pubblico di piccole raccolte giornaliere, cui il pubblico può accedere e prenderne per quantità non superiori ai «tre pasti per famiglia».

Un percorso alternativo non viene certificato come kasher, e quindi non trova distribuzione presso la GDO Israeliana ${ }^{87}$. Il prodotto, inoltre, non può essere esportato. Se esportato, lo è senza certificazione kasher, per cui non appetibile ad un pubblico Ebraico osservante nella diaspora, stimato in almeno due milioni di persone negli Stati Uniti d'America e circa trecentomila in Europa ${ }^{88}$, anche se senza implicazioni per un pubblico non Ebraico. Interessante anche la gestione delle eventuali eccedenze: i prodotti della Shemita godono di sacralità, per cui la loro distruzione è proibita; possono essere, nuovamente, esposti al pubblico e/o agli animali, ma non distrutti in modo disonorevole, tipo l'immondizia normale.

\section{Cosa c'entra tutto ciò con i sopracitati Obiettivi 2 e 15 ?}

Queste soluzioni vanno inquadrate nelle loro attuali prospettive: la gestione di "doni, decime, angolo, spigolatura, dimenticanza" o delle produzioni della Shemità non implicano necessariamente una buona volontà o magnanimità da parte dei produttori/agricoltori; si tratta di gestire una quota parte di prodotti non destinati al libero consumo da un

85. https://www.ynet.co.il/articles/0,7340,L-4180860,00.html (26.01.12); https://www. srugim.co.il (26.01.12).

86. M. Maimonide (Rambam) (1138-1204), Mishne Torah, Melachim-Milchamot, 6:10.

87. Per sinteticità, non entro nel merito dei meccanismi alternativi per le produzioni durante la Shemità: heter mechirà; yevul nochri; yevul admot haarava, etc. Ho preferito dare precedenza al meccanismo di più rilevanza sociale e che, apparentemente, gode anche del più largo favore da parte del pubblico osservante Ebraico Israeliano.

88. Si tratta di stime personali, derivate da fonti spesso disparate; non scientificamente riorganizzati. 
punto di vista Ebraico in quanto o destinati ad un pubblico particolare e/o da gestire in modo particolare (Shemita); come tali, si considerano proibiti e, malgrado sia difficile immaginarlo, esattamente alla stessa stregua di maiale e gamberetti.

Il loro scorporo, a favore dei più bisognosi, l'immissione sul mercato a prezzi di costo-manodopera, etc., risolvono ogni problema? Forse non del tutto, o forse no; ma consapevolmente o meno, volenti o nolenti, estendono a tutti, in Israele, perlomeno, un concetto di minima solidarietà sociale.

\section{Un tentativo di conclusione}

L'Ebraismo definitivamente ha una posizione sugli animali:

- consapevolezza del loro essere viventi;

- consapevolezza del loro poter soffrire;

- consapevolezza dei loro bisogni e necessità.

È imperativo ad essere misericordiosi nei loro confronti.

L'Ebraismo definitivamente ha una posizione anche sull'ambiente:

- consapevolezza della necessità alla sua protezione;

- consapevolezza che non è nostro;

- consapevolezza della irrimediabilità dei danni: «presta la tua attenzione a non danneggiare ed a non distruggere il mio mondo; poiché se l'avrai danneggiato, non c'è chi lo riparerà dopo di te» ${ }^{89}$.

Attuale come non mai in questo periodo di discussioni feroci su come e quanto si possa ancora (ridurre di) inquinare.

L'Ebraismo definitivamente ha una posizione sulla corresponsabilità sociale; sulla relatività della proprietà terriera e sul suo sfruttamento.

Ma anche sull'umana debolezza relativamente alla spontanea generosità; da cui un meccanismo di obbligatorietà di comportamenti e strutture finalizzate ad una certa redistribuzione e compartecipazione dei beni di prima necessità.

L'Ebraismo definitivamente ha una posizione su: animali, ambiente, società, in modo solo apparentemente discordante: 
- dominio misericordioso sugli animali;

- conservazione del creato;

- rispetto e compassione del prossimo.

Il nodo di collegamento è rappresentato dall'utilizzo consapevole del Creato: utilizzo pietoso degli animali, con divieto alla loro sofferenza, forse anche non solo fisica; divieto allo spreco ed alla distruzione, al deturpamento; condivisione delle risorse con i meno abbienti.

Ma anche consapevolezza che «il compimento di una buona azione, ne genera un'altra» ${ }^{90}$.

Nel Libro di Ruth (circa xi secolo a.C.), letto generalmente durante la festività di Shavuot-Pentecoste, si legge la storia di Ruth: Moabita e vedova; non Ebrea, suocera di Naomi, Ebrea e vedova anch'essa. Ruth al tempo della mietitura del grano si accodò ai mietitori nel campo di Boaz, parente del marito defunto di Naomi, appunto per spigolare e preparare le scorte per sé e per la vedova Naomi.

Boaz, venutolo a sapere, non solo permise a Ruth di spigolare il grano, invitandola a non andare da altri, ma disse ai suoi operai di «dimenticarne di più» ${ }^{91}$.

Boaz, alla fine, sposò Ruth. «Boaz generò Oved; Oved generò Yshai; Yshai generò David» ${ }^{92}$.

E i giorni che regnò David furono 40 anni; a Hevron regnò

7 anni ed a Gerusalemme regnò 33 anni. ${ }^{93}$

Il più grande Re di Israele derivò da una donna convertita all'Ebraismo e dall'osservanza di una mitzwah molto distante da quelle che potrebbero essere le eroiche gesta di altre dinastie.

90. Mishna, Massechet Avot - Le Massime dei Padri; 4:2.

91. Ruth, 2:15.

92. Ruth, 4:22.

93. Melachim - Re - I - 2:11. 
Gli obiettivi dello sviluppo sostenibile nell'Enciclica Laudato si'

Giuseppe Zeppegno

L'enciclica Laudato si' (Ls), pubblicata il 24 maggio 2015 da Papa Francesco, prende il nome dal Cantico delle creature di Francesco d'Assisi, santo più volte citato nel corso del documento e indicato espressamente come «'esempio per eccellenza della cura per ciò che è debole e di una ecologia integrale, vissuta con gioia e autenticità [...]. In lui si riscontra fino a che punto sono inseparabili la preoccupazione per la natura, la giustizia verso i poveri, l'impegno nella società e la pace interiore» (Ls 10). Fin dai primi due paragrafi si evidenzia che San Francesco ricordava nel Cantico che «la nostra casa comune è anche come una sorella, con la quale condividiamo l'esistenza, e come una madre bella che ci accoglie tra le sue braccia» (Ls 1). Papa Francesco invita a riflettere scrivendo che

questa sorella protesta per il male che le provochiamo, a causa dell'uso irresponsabile e dell'abuso dei beni che Dio ha posto in lei. Siamo cresciuti pensando di essere suoi proprietari e dominatori, autorizzati a saccheggiarla. La violenza che c'è nel cuore umano ferito dal peccato si manifesta anche nei sintomi di malattia che avvertiamo nel suolo, nell'acqua, nell'aria e negli esseri viventi. Per que- 
sto, fra i poveri più abbandonati e maltrattati, c'è la nostra oppressa e devastata terra, che "geme e soffre le doglie del parto" (Rm 8,22). Dimentichiamo che noi stessi siamo terra (cfr. Gen 2,7). Il nostro stesso corpo è costituito dagli elementi del pianeta, la sua aria è quella che ci dà il respiro e la sua acqua ci vivifica e ristora. (Ls 2)

Questa preoccupazione era già stata mossa dal Papa nella sua prima enciclica, la Lumen fide $i^{1}$. Al paragrafo 55 aveva infatti asserito:

La fede nel rivelarci l'amore di Dio creatore, ci fa rispettare maggiormente la natura, facendoci riconoscere in essa una grammatica da lui scritta e una dimora a noi affidata, perché sia coltivata e custodita; ci aiuta a trovare modelli di sviluppo che non si basino solo sull'utilità e il profitto, ma che considerino il creato come dono, cui tutti siamo debitori.

Questa considerazione non era peraltro nuova. Rispondeva ad una precisa attenzione già presente in alcuni scritti dei primi secoli dell'era cristiana ed era stata ampiamente riconsiderata in questi ultimi decenni. In questa linea, risulta centrale nella Ls una domanda che troviamo al paragrafo 160: «Che tipo di mondo desideriamo trasmettere a coloro che verranno dopo di noi, ai bambini che ora stanno crescendo?». Il Papa aggiunge che è una domanda che non riguarda solo l'ambiente perché «quando ci interroghiamo circa il mondo che vogliamo lasciare ci riferiamo soprattutto al suo orientamento generale, al suo senso, ai suoi valori». Devono pulsare quindi alcune domanda di fondo: «A che scopo passiamo da questo mondo? Per quale fine siamo venuti in questa vita? Per che scopo lavoriamo e lottiamo? Perché questa terra ha bisogno di noi?». Non basta, infatti, «che dobbiamo preoccuparci per le future generazioni. Occorre rendersi conto che quello che c'è in gioco è la dignità di noi stessi. Siamo noi i primi interessati a trasmettere un pianeta abitabile per l'umanità che verrà dopo di noi. È un dramma per noi stessi, perché ciò chiama in causa il significato del nostro passaggio su questa terra».

Nei due paragrafi successivi il Papa precisa che «le previsioni catastrofiche ormai non si possono più guardare con

1. Francesco, Lett. Enc. Lumen fidei (29 giugno 2013): AAS 105(2013), 555-596. 
disprezzo e ironia» perché «potremmo lasciare alle prossime generazioni troppe macerie, deserti e sporcizia. Il ritmo di consumo, di spreco e di alterazione dell'ambiente ha superato le possibilità del pianeta, in maniera tale che lo stile di vita attuale, essendo insostenibile, può sfociare solamente in catastrofi, come di fatto sta già avvenendo periodicamente in diverse regioni». Tale squilibrio «dipende da ciò che facciamo ora, soprattutto se pensiamo alla responsabilità che ci attribuiranno coloro che dovranno sopportare le peggiori conseguenze. La difficoltà a prendere sul serio questa sfida è legata ad un deterioramento etico e culturale, che accompagna quello ecologico». Nota inoltre che gli uomini e le donne in questo mondo postmoderno «corrono il rischio permanente di diventare profondamente individualisti». Molti problemi sociali, infatti, «sono da porre in relazione con la ricerca egoistica della soddisfazione immediata, con le crisi dei legami familiari e sociali, con le difficoltà a riconoscere l'altro. Molte volte si è di fronte ad un consumo eccessivo e miope dei genitori che danneggia i figli, che trovano sempre più difficoltà ad acquistare una casa propria e a fondare una famiglia». Manca inoltre la capacità «di pensare seriamente alle future generazioni» perché siano incapaci «di ampliare l'orizzonte delle nostre preoccupazioni e pensare a quanti rimangono esclusi dallo sviluppo. Non perdiamoci a immaginare i poveri del futuro, è sufficiente che ricordiamo i poveri di oggi, che hanno pochi anni da vivere su questa terra e non possono continuare ad aspettare».

Questa preoccupazione ripercorre in modo organico i sei capitoli di cui consta l'enciclica. Nel primo infatti si conduce una attenta e scientificamente provata analisi della situazione attuale. Nel secondo, ponendo particolare attenzione ai racconti biblici della creazione e del peccato originale, rileva che nell'insegnamento scritturistico ed ecclesiale è centrale l'insistenza sulla bontà del creato e sul ruolo che l'uomo deve assumere per custodirlo. Il capitolo terzo, in dialogo con la filosofia e le scienze umane, si occupa delle cause per cui si è giunti alla situazione attuale. Il capitolo successivo tratteggia gli elementi cardine di un'ecologia integrale capace di orientare un nuovo modo di essere dell'uomo nel creato. Il capito quinto, senza presunzione alcuna di sostituirsi alle diverse componenti della società, sostiene la necessità di un 
rinnovamento capace di coniugare politica ed economia, religioni e scienze, economia e impresa per il bene integrale dell'uomo e di questo nostro mondo globalizzato. Infine, l'ultimo capitolo delinea gli elementi essenziali di una spiritualità ecologica nella convinzione che «quando le persone diventano autoreferenziali e si isolano nella loro coscienza, accrescono la propria avidità. Più il cuore della persona è vuoto, più ha bisogno di oggetti da comprare, possedere e consumare. In tale contesto non sembra possibile che qualcuno accetti che la realtà gli ponga un limite. In questo orizzonte non esiste nemmeno un vero bene comune» (Ls 204).

Sono due gli argomenti principali dell'enciclica: la contemplazione per la bellezza del creato e il gemito di sofferenza che sgorga dalla creazione e da tante creature ingiustamente vilipese. Il Pontefice osserva:

Se noi ci accostiamo alla natura e all'ambiente senza questa apertura allo stupore e alla meraviglia, se non parliamo più il linguaggio della fraternità e della bellezza nella nostra relazione con il mondo, i nostri atteggiamenti saranno quelli del dominatore, del consumatore o del mero sfruttatore delle risorse naturali, incapace di porre un limite ai suoi interessi immediati. Viceversa, se noi ci sentiamo intimamente uniti a tutto ciò che esiste, la sobrietà e la cura scaturiranno in maniera spontanea. (Ls 11)

Il tema della bellezza del creato - ampiamente trattato, con sfumature diverse, da numerosi autori - è caro a Papa Francesco. Ne parlò diffusamente già il 19 marzo 2013 nell'omelia della Messa d'inizio pontificato dove invitò a custodire Cristo nella vita per custodire al medesimo tempo gli altri e il creato: «La vocazione del custodire non riguarda solamente noi cristiani» perché «ha una dimensione che precede e che è semplicemente umana, riguarda tutti». La custodia del creato "come ci viene detto nel Libro della Genesi e come ci ha mostrato san Francesco d'Assisi: è l'avere rispetto per ogni creatura di Dio e per l'ambiente in cui viviamo». Impegna quindi a «custodire la gente, l'aver cura di tutti, di ogni persona, con amore, specialmente dei bambini, dei vecchi, di coloro che sono più fragili e che spesso sono nella periferia del nostro cuore». All'interno della famiglia «i coniugi si custodiscono reciprocamente, poi come genitori si prendono cura dei figli, e col tempo anche i figli diventano 
custodi dei genitori. È il vivere con sincerità le amicizie, che sono un reciproco custodirsi nella confidenza, nel rispetto e nel bene. In fondo, tutto è affidato alla custodia dell'uomo, ed è una responsabilità che ci riguarda tutti. Siate custodi dei doni di Dio!»².

Nella medesima omelia mise in guardia anche dall'Erode che è in noi ricordando che l'uomo se viene meno alla responsabilità di custodire non solo il creato ma anche $\mathrm{i}$ fratelli «allora trova spazio la distruzione e il cuore inaridisce». Chiede, pertanto, a tutti coloro che occupano ruoli di responsabilità di essere custodi «della creazione, del disegno di Dio iscritto nella natura, custodi dell'altro, dell'ambiente». Custodire - precisa - «vuol dire allora vigilare sui nostri sentimenti, sul nostro cuore, perché è proprio da lì che escono le intenzioni buone e cattive: quelle che costruiscono e quelle che distruggono! Non dobbiamo avere paura della bontà, anzi neanche della tenerezza! E qui aggiungo, allora, un'ulteriore annotazione: il prendersi cura, il custodire chiede bontà, chiede di essere vissuto con tenerezza $»^{3}$.

Le stesse argomentazioni tornano nel primo capitolo della Laudato si', dove sono elencati gli attentati che costantemente il creato subisce: inquinamento, cambiamenti climatici, uso incontrollato dell'acqua e perdita di biodiversità. Per ammissione di numerosi scienziati, queste questioni sono affrontate nell'enciclica con estrema competenza scientifica. Va precisato che il Papa non enuncia i problemi per condurre un'accusa sterile nei confronti di chi provoca i disastri ambientali. Il suo obiettivo, infatti, è di aiutare a «prendere dolorosa coscienza» della realtà per «osare trasformare in sofferenza personale quello che accade al mondo, e così riconoscere qual è il contributo che ciascuno può portare» (Ls 19).

Al paragrafo 22 annota: «questi problemi sono intimamente legati alla cultura dello scarto, che colpisce tanto gli esseri umani esclusi quanto le cose che si trasformano velocemente in spazzatura». Al paragrafo 43 riprende questo discorso rilevando: «se teniamo conto del fatto che anche

2. Id., «Omelia della Messa d'inizio pontificato», 19 Marzo 2013, inhttp://www.vatican. $\mathrm{va} /$ content/francesco/it/homilies/2013/documents/papa-francesco_20130319_omelia-inizio-pontificato.html [12 marzo 2020].

3. Ibid. 
l'essere umano è una creatura di questo mondo, che ha diritto a vivere e ad essere felice, e inoltre ha una speciale dignità, non possiamo tralasciare di considerare gli effetti del degrado ambientale, dell'attuale modello di sviluppo e della cultura dello scarto sulla vita delle persone». Enuclea subito dopo la disordinata crescita di tante città diventate invivibili (Ls 44), la privatizzazione degli spazi verdi (Ls 45), "gli effetti occupazionali di alcune innovazioni tecnologiche, l'esclusione sociale, la disuguaglianza nella disponibilità e nel consumo dell'energia e di altri servizi, la frammentazione sociale, l'aumento della violenza e il sorgere di nuove forme di aggressività sociale, il narcotraffico e il consumo crescente di droghe fra i più giovani, la perdita di identità» (Ls 46).

È facile notare che volutamente il discorso si sposta dall'ambiente all'uomo per dimostrare «come la crescita degli ultimi due secoli non ha significato in tutti i suoi aspetti un vero progresso integrale e un miglioramento della qualità della vita. Alcuni di questi segni sono allo stesso tempo sintomi di un vero degrado sociale, di una silenziosa rottura dei legami di integrazione e di comunione sociale» (Ls 46). Contribuiscono a questo impoverimento molti fattori.

1. Le dinamiche dei media e del mondo digitale che sono sempre più onnipresenti ma «non favoriscono lo sviluppo di una capacità di vivere con sapienza, di pensare in profondità, di amare con generosità» (Ls 47).

2. L'iniquità con cui vengono affrontati i drammi dei poveri del mondo visto che «invece di risolvere i problemi dei poveri e pensare a un mondo diverso, alcuni si limitano a proporre una riduzione della natalità. Non mancano pressioni internazionali sui Paesi in via di sviluppo che condizionano gli aiuti economici a determinate politiche di "salute riproduttiva" (Ls 50).

3. Il "debito ecologico" tra il Nord e il Sud del mondo determinato dalle «esportazioni di alcune materie prime per soddisfare i mercati nel Nord industrializzato hanno prodotto danni locali, come l'inquinamento da mercurio nelle miniere d'oro o da diossido di zolfo in quelle di rame». Bisogna poi «calcolare l'uso dello spazio ambientale di tutto il pianeta per depositare rifiuti gassosi che sono andati accumulandosi durante due secoli e hanno generato una 
situazione che ora colpisce tutti i Paesi del mondo». Cita il riscaldamento che ha gravi ripercussioni sui paesi più poveri della terra». A questo - aggiunge - «si uniscono i danni causati dall'esportazione verso i Paesi in via di sviluppo di rifiuti solidi e liquidi tossici e dall'attività inquinante di imprese che fanno nei Paesi meno sviluppati ciò che non possono fare nei Paesi che apportano loro capitale» (Ls 51).

4. Lo sfruttamento dei popoli del Terzo Mondo che hanno «le riserve più importanti della biosfera» ma che continuano, loro malgrado, «ad alimentare lo sviluppo dei Paesi più ricchi a prezzo del loro presente e del loro futuro» (Ls 52). Queste ingiustizie sono contrastate con reazioni deboli e pressoché insignificanti (Ls 53-59) anche perché non si vuole considerarle con la giusta attenzione. "Da un estremo, alcuni sostengono ad ogni costo il mito del progresso e affermano che i problemi ecologici si risolveranno semplicemente con nuove applicazioni tecniche, senza considerazioni etiche né cambiamenti di fondo. Dall'altro estremo, altri ritengono che la specie umana, con qualunque suo intervento, possa essere solo una minaccia e compromettere l'ecosistema mondiale, per cui conviene ridurre la sua presenza sul pianeta e impedirle ogni tipo di intervento. Fra questi estremi, la riflessione dovrebbe identificare possibili scenari futuri, perché non c’è un'unica via di soluzione. Questo lascerebbe spazio a una varietà di apporti che potrebbero entrare in dialogo in vista di risposte integrali» (Ls 60). Non bisogna arrendersi «La speranza ci invita a riconoscere che c'è sempre una via di uscita, che possiamo sempre cambiare rotta, che possiamo sempre fare qualcosa per risolvere i problemi» (Ls 61).

\section{Viviamo in un mondo sovrappopolato?}

Papa Francesco evidenzia che alcune correnti ambientaliste da decenni sostengono che il mondo è sull'orlo del baratro a causa della sovrappopolazione. È doveroso rilevare che tra quanti si sono pronunciati con toni allarmistici a proposito della crescita demografica dell'ultimo secolo troviamo anche illustri personaggi. I "rimedi" che propongono per arginare il temuto incremento demografico hanno spesso dell'incredibile. Il principe Filippo di Edimburgo ad esempio ha dichiarato ad un'agenzia di stampa tedesca: «Nel caso 
io rinasca, mi piacerebbe essere un virus letale, così da contribuire a risolvere il problema della sovrappopolazione» ${ }^{4}$.

Konrad Lorenz, noto fondatore dell'etologia e premio Nobel per la medicina nel 1973, ha asserito: "Contro la sovrappopolazione l'umanità non ha mai intrapreso nulla di sensato. Si potrebbe perciò avere una certa simpatia per l'AIDS. È una minaccia che potrebbe decimare l'umanità ed impedirle altre imprese minacciose $»^{5}$.

Il famoso ambientalista Jacques Cousteau, a seguito del tifone che nel 1991 ha devasto il Bangladesh, ha affermato: «Non date la colpa al mare. La vera tragedia del Bangladesh sono gli uomini, una popolazione incontenibile [...]. Dovremo essere in 700 milioni in tutto, allora si che la vita sulla Terra diventerebbe paradisiaca. Per stabilizzare la popolazione mondiale, dobbiamo eliminare 350 mila persone al giorno. È una cosa terribile a dirsi, ma è anche peggio non dirla» ${ }^{6}$.

Ancora nel Terzo Millennio sono molti coloro che si mantengono sui binari dell'ideologia catastrofista e auspicano una repentina diminuzione della popolazione. A conferma cito Eric R. Pianka, zoologo evoluzionista dell'Università di Austin in Texas. È sua convinzione che sulla Terra può vivere decorosamente solo il $10 \%$ dell'attuale popolazione. Durante la relazione tenuta al Texas Academy of Science nel marzo del 2006, ha proposto di modificare in laboratorio il virus dell'Ebola per renderlo ancora più contagioso, quindi atto a ridurre drasticamente e in breve tempo gli abitanti del pianeta ${ }^{7}$.

In un editoriale del Corriere della Sera dal titolo «Una modernità fuori misura» il noto politologo Giovanni Sartori si è sentito in dovere di esporre l'opinione secondo cui «stiamo inquinando l'atmosfera, stiamo avvelenando l'aria che respiriamo e, al contempo, stiamo destabilizzando il clima [...]. L'unica cura ancora a nostra disposizione è di ridurre la popolazione e con essa ridurre l'emissione di gas

4. Prince Philip, riportato da Deuteche Press Agentur, August 1988.

5. M. Blondet., Hitler, un si all'aborto che precorre Il Cairo, «Avvenire», 14 agosto 1994, 4.

6. F. Malaspina, I limiti del sottosviluppo, «Zenit», 8 luglio 2008.

7. P.J. Watson, Top scientist advocates mass culling of $90 \%$ human population, «PrisonPlanet», 3 aprile 2006. 
serra e la conseguente concentrazione di anidride carbonica nell'atmosfera» ${ }^{8}$.

Molti scienziati hanno raggiunto durante le loro ricerche ben altre consapevolezze. Dati alla mano, osservano con sempre maggiore convinzione che gli squilibri ambientali non sono determinati dalla densità della popolazione, ma dagli inadeguati e ingiusti sistemi sociali vigenti. Menziono tra i tanti che si sono impegnati a sviscerare scientificamente la questione, il premio Nobel per l'economia Angus Deaton. Nel libro La grande fug $a^{9}$, denota che, a dispetto dei profeti di sventura, nonostante l'aumento della popolazione, si è registrata la riduzione della mortalità e non si è verificata la tanto temuta carestia mondiale.

Osservazioni simili propone anche Erle C. Ellis, professore associato di geografia e sistemi ambientali presso l'Università del Maryland. In un articolo comparso sul New York Times del 13 settembre 2013 con il titolo Overpopulation Is Not the Problem rileva che l'idea secondo cui gli esseri umani stanno superando la capacità di carico naturale della terra è una sciocchezza dovuta all'incapacità di comprendere il vero senso dell'ecologia dei sistemi umani che fin dalla preistoria hanno usato la tecnica e gli ecosistemi ingegnerizzati per sostenere le popolazioni con una potenzialità superiore ai mezzi naturali ${ }^{10}$.

Anche Umberto Veronesi nella rubrica che teneva abitualmente sul settimanale Oggi ha affermato che i catastrofisti sono in malafede "perché grazie alla scienza c'è uno sviluppo "sostenibile" che può consentire la vita a tutti quanti» ${ }^{11}$.

È importante notare che l'Assemblea Generale delle Nazioni Unite dal settembre scorso ha reso operativi gli "Obiettivi di sviluppo sostenibile" che dovranno essere realizzati entro il 2030 con la collaborazione di Istituzioni statali, organizzazioni della società civile, accademici e scienziati. Secondo la nobile istituzione, sono 17 gli intenti da attuare. Prevedono il ripristino degli ecosistemi, lo sradicamento della povertà, la possibilità di garantire vita sana,

8. G. Sartori, Una modernità fuori misura, «Corriere della Sera», 25 novembre 2013.

9. A. Deaton, La grande fuga, il Mulino, Bologna 2015.

10. E. C. Ellis, Overpopulation Is Not the Problem, «New York Times», 13 settembre 2013.

11. U. Veronesi, Un mondo senza fame? Con la scienza si può, «Oggi», 26 settembre 2014. 
pace, educazione, dignità, l'accesso alle fonti energetiche, un adeguato nutrimento e acqua per tutti. Purtroppo, però nel documento permane la convinzione che sia necessario ridurre le nascite. Il protocollo ideato prevede pertanto la diffusione della contraccezione e dell'aborto chimico e chirurgico.

Papa Francesco dal canto suo scrive al paragrafo 50 dell'enciclica: «Invece di risolvere i problemi dei poveri e pensare a un mondo diverso, alcuni si limitano a proporre una riduzione della natalità. Non mancano pressioni internazionali sui Paesi in via di sviluppo che condizionano gli aiuti economici a determinate politiche di "salute riproduttiva"». Rileva che "se è vero che l'ineguale distribuzione della popolazione e delle risorse disponibili crea ostacoli allo sviluppo e ad un uso sostenibile dell'ambiente, va riconosciuto che la crescita demografica è pienamente compatibile con uno sviluppo integrale e solidale"». Pertanto «incolpare l'incremento demografico e non il consumismo estremo e selettivo di alcuni, è un modo per non affrontare i problemi. Si pretende così di legittimare l'attuale modello distributivo, in cui una minoranza si crede in diritto di consumare in una proporzione che sarebbe impossibile generalizzare». Questo perché «il pianeta non potrebbe nemmeno contenere i rifiuti di un simile consumo. Inoltre, sappiamo che si spreca approssimativamente un terzo degli alimenti che si producono, e "il cibo che si butta via è come se lo si rubasse dalla mensa del povero"». È allora doveroso «prestare attenzione allo squilibrio nella distribuzione della popolazione sul territorio, sia a livello nazionale sia a livello globale, perché l'aumento del consumo porterebbe a situazioni regionali complesse, per le combinazioni di problemi legati all'inquinamento ambientale, ai trasporti, allo smaltimento dei rifiuti, alla perdita di risorse, alla qualità della vita».

Il 20 novembre 2014, alcuni mesi prima della pubblicazione della Laudato si, nel discorso tenuto alla sede romana della Fao, aveva dato chiare indicazioni per risolvere il problema della fame nel mondo senza arrivare a minacciare la vita umana nascente. È opportuno rileggere queste sue affermazioni. Le sintetizzo in quattro punti. Possono diventare fonte di meditazione e di verifica del modo di gestire $\mathrm{i}$ beni che abbiamo a disposizione. 
1. Evitare lo spreco. Nonostante ci sia cibo per tutti, non tutti possono mangiare perché in molte parti della terra continua ad esserci «lo spreco, lo scarto, il consumo eccessivo e l'uso di alimenti per altri fini» mentre in altre parti continua ad esserci la fame. È questa «la prima sfida che bisogna superare».

2. Favorire la solidarietà. Una seconda sfida è data dalla mancanza di solidarietà perché le «nostre società sono caratterizzate da un crescente individualismo e dalla divisione; ciò finisce col privare i più deboli di una vita degna e con il provocare rivolte contro le istituzioni». Infatti, se manca la solidarietà, tutti ne risentono perché «è l'atteggiamento che rende le persone capaci di andare incontro all'altro e di fondare i propri rapporti reciproci su quel sentimento di fratellanza che va al di là delle differenze e dei limiti, e spinge a cercare insieme il bene comune».

3. Rispettarsi reciprocamente. «Gli esseri umani, nella misura in cui prendono coscienza di essere parte responsabile del disegno della creazione, diventano capaci di rispettarsi reciprocamente, invece di combattere tra loro, danneggiando e impoverendo il pianeta». Allo stesso modo «agli Stati, concepiti come comunità di persone e di popoli, viene chiesto di agire di comune accordo, di essere disposti ad aiutarsi gli uni gli altri mediante i principi e le norme che il diritto internazionale mette a loro disposizione» Il Papa prosegue osservando: «una fonte inesauribile d'ispirazione è la legge naturale, iscritta nel cuore umano, che parla un linguaggio che tutti possono capire: amore, giustizia, pace, elementi inseparabili tra loro».

4. Attenzione ai poveri. «Ogni donna, uomo, bambino, anziano deve poter contare su queste garanzie dovunque. Ed è dovere di ogni Stato, attento al benessere dei suoi cittadini, sottoscriverle senza riserve, e preoccuparsi della loro applicazione. Ciò richiede perseveranza e sostegno». Dal canto suo «la Chiesa cattolica cerca di offrire anche in questo campo il proprio contributo, mediante un'attenzione costante alla vita dei poveri, dei bisognosi in ogni parte del pianeta». La Santa Sede interviene al riguardo «nelle organizzazioni internazionali e con i suoi molteplici documenti e dichiarazioni. S'intende in tal modo contribuire a identificare e adottare i criteri che devono realizzare lo 
sviluppo di un sistema internazionale equo. Sono criteri che, sul piano etico, si basano su pilastri come la verità, la libertà, la giustizia e la solidarietà; allo stesso tempo, in campo giuridico, questi stessi criteri includono la relazione tra il diritto all'alimentazione e il diritto alla vita e a un'esistenza degna» ${ }^{12}$.

\section{Il Vangelo della Creazione}

Il secondo capitolo della Laudato si' occupa i paragrafi 62100 e propone sette piste di riflessione. Nella prima Papa Francesco ricorda che, anche se ha l'intento di rivolgersi a tutta la famiglia umana, non può esimersi dal proporre una lettura credente della creazione. Lo fa nella consapevolezza che ogni uomo può trarre non solo dalla scienza, ma da ogni saggezza, anche quella religiosa, strumenti utili per individuare soluzioni atte a contrastare la crisi ambientale (Ls 63). Nella seconda specifica che la tradizione biblica indica «motivazioni alte per prendersi cura della natura e dei fratelli e sorelle più fragili» (Ls 65). Gli stessi racconti della creazione «contengono, nel loro linguaggio simbolico e narrativo, profondi insegnamenti sull'esistenza umana e la sua realtà storica». Suggeriscono che l'esistenza umana è fondata su tre relazioni fondamentali: la relazione con Dio, con il prossimo e con la terra. Secondo la Bibbia - continua - «queste tre relazioni vitali sono rotte, non solo fuori, ma anche dentro di noi. Questa rottura è il peccato. L'armonia tra il Creatore, l'umanità e tutto il creato è stata distrutta per avere noi preteso di prendere il posto di Dio, rifiutando di riconoscerci come creature limitate». È un fatto ha distorto anche il mandato di soggiogare, coltivare e custodire la terra (cfr. Gen 1,28; 2,15) e ha distrutto «la relazione originariamente armonica tra essere umano e natura» trasformandola in conflitto (cfr. Gen 3,17-19). Diventa allora comprensibile il fatto che «l'armonia che san Francesco d'Assisi viveva con tutte le creature sia stata interpretata come una guarigione di tale rottura. San Bonaventura disse che attraverso la riconciliazione universale con tutte le

12. Francesco, «Discorso in occasione alla II Conferenza Internazionale sulla nutrizione», Sede della Fao, Roma 24 novembre 2014, in http://www.vatican.va/content/francesco/it/speeches/2014/november/documents/papa-francesco_20141120_visita-fao.Ht ml [15 marzo 2019]. 
creature in qualche modo Francesco era riportato allo stato di innocenza originaria» (par. 66). A differenza di quanto spesso si asserisce, la tradizione ebraico-cristiana non invita a sfruttare in modo selvaggio la natura, ma a "coltivare e custodire" il giardino del mondo (cfr. Gen 2,15). Mentre "coltivare" significa arare o lavorare un terreno, "custodire" vuol dire proteggere, curare, preservare, conservare, vigilare». Ne deriva «una relazione di reciprocità responsabile tra essere umano e natura». Ogni comunità può servirsi dei beni della terra per la propria sopravvivenza ricordando però che "“del Signore è la terra" (Sal 24,1), a Lui appartiene "la terra e quanto essa contiene" (Dt 10,14). Perciò Dio nega ogni pretesa di proprietà assoluta: "Le terre non si potranno vendere per sempre, perché la terra è mia e voi siete presso di me come forestieri e ospiti" (Lv 25,23)» (Ls 67). Tale responsabilità implica il rispetto "delle leggi della natura e i delicati equilibri tra gli esseri di questo mondo» (Ls 68) perché il rifiuto di coltivare e mantenere una relazione corretta con l'ambiente distrugge la relazione interiore con se stessi, gli altri e Dio oltre che con la terra: giustizia non abita più sulla terra, la Bibbia ci dice che tutta la vita è in pericolo. Questo è ciò che ci insegna il racconto di Noè, quando Dio minaccia di spazzare via l'umanità per la sua persistente incapacità di vivere all'altezza delle esigenze della giustizia e della pace (Gen 6,13)»(Ls 70). I salmi e gli scritti dei profeti invitano invece ad accogliere l'amore divino che si manifesta nell'armonia del Creato, a lodarlo e adorarlo (Ls 72-73). Se così non fosse, «finiremmo per adorare altre potenze del mondo, o ci collocheremmo al posto del Signore, fino a pretendere di calpestare la realtà creata da Lui senza conoscere limite». Invece «il modo migliore per collocare l'essere umano al suo posto e mettere fine alla sua pretesa di essere un dominatore assoluto della terra, è ritornare a proporre la figura di un Padre creatore e unico padrone del mondo, perché altrimenti l'essere umano tenderà sempre a voler imporre alla realtà le proprie leggi e i propri interessi» (Ls 75). Nella terza pista di riflessione il Papa invita a riconoscere che «per la tradizione giudeocristiana, dire "creazione" è più che dire natura, perché ha a che vedere con un progetto dell'amore di Dio, dove ogni creatura ha un valore e un significato». La natura è così 
intesa come un sistema che «si analizza, si comprende e si gestisce, ma la creazione può essere compresa solo come un dono che scaturisce dalla mano aperta del Padre di tutti, come una realtà illuminata dall'amore che ci convoca ad una comunione universale» (Ls 76). Il pensiero ebraico-cristiano inoltre «ha demitizzato la natura. Senza smettere di ammirarla per il suo splendore e la sua immensità, non le ha più attribuito un carattere divino». Ne deriva che «un ritorno alla natura non può essere a scapito della libertà e della responsabilità dell'essere umano». Quindi se riconosciamo la fragilità della natura e le capacità che il Creatore ci ha dato, possiamo «porre fine al mito moderno del progresso materiale illimitato. Un mondo fragile, con un essere umano al quale Dio ne affida la cura, interpella la nostra intelligenza per riconoscere come dovremmo orientare, coltivare e limitare il nostro potere» (Ls 78). La fiducia che Dio ci ha accordato può anche essere spesa male e «può aggiungere nuovi mali, nuove cause di sofferenza e momenti di vero arretramento» (Ls 79). Ciò nonostante egli «vuole agire con noi e contare sulla nostra collaborazione» e trae «qualcosa di buono dai mali che noi compiamo, perché "lo Spirito Santo possiede un'inventiva infinita, propria della mente divina, che sa provvedere a sciogliere i nodi delle vicende umane anche più complesse e impenetrabili”»(Ls 80). Nella quarta pista di riflessione invita a riconoscere che «ogni creatura ha una funzione e nessuna è superflua» (Ls 84) ed ogni cosa è segno del progetto amoroso di Dio (Ls 86). Questa consapevolezza spinge naturalmente ad «adorare il Signore per tutte le sue creature» come fece san Francesco d'Assisi con il suo bellissimo cantico (Ls 87). Ringraziare il Signore però non equivale a idolatrare le sue creature perché «le cose di questo mondo non possiedono la pienezza di Dio» (Ls 88). Nella quinta pista di riflessione il Papa evidenzia che ogni realtà creata trova il suo senso in Dio e il mancare di rispetto anche ad una sola creatura diventa fonte di degradazione e di perturbazione della dignità per l'uomo. Questa consapevolezza però non deve portare ad «equiparare tutti gli esseri viventi e togliere all'essere umano quel valore peculiare che implica allo stesso tempo una tremenda responsabilità. E nemmeno comporta una divinizzazione della terra, che ci priverebbe della chiamata a collaborare con essa e a proteggere la sua fragilità» (Ls 90). La cura che 
si deve avere per tutti gli esseri viventi non ci deve distogliere quindi dall'impegno a «difendere la pari dignità tra gli esseri umani», a indignarci per «le enormi disuguaglianze che esistono tra di noi», ad accorgerci di chi si trascina «in una miseria degradante, senza reali possibilità di miglioramento, mentre altri non sanno nemmeno che farsene di ciò che possiedono [...]. Continuiamo nei fatti ad ammettere che alcuni si sentano più umani di altri, come se fossero nati con maggiori diritti» (Ls 90). Il Papa per aiutare a capire questa aporia pone un esempio: «È evidente l'incoerenza di chi lotta contro il traffico di animali a rischio di estinzione, ma rimane del tutto indifferente davanti alla tratta di persone, si disinteressa dei poveri, o è determinato a distruggere un altro essere umano che non gli è gradito. Ciò mette a rischio il senso della lotta per l'ambiente» (Ls 91).

Nella sesta pista di riflessione tratta il tema della destinazione comune dei beni, tema tipico della morale sociale. Osserva che la terrà è un bene di tutti «perché Dio ha creato il mondo per tutti» (Ls 93). La proprietà pertanto non è un diritto assoluto e intoccabile perché su ogni proprietà «grava sempre un'ipoteca sociale» (Ls 93). Chi possiede una parte dell'ambiente deve offrirlo cioè a beneficio di tutti perché «se non lo facciamo, ci carichiamo sulla coscienza il peso di negare l'esistenza degli altri. Per questo i Vescovi della Nuova Zelanda si sono chiesti che cosa significa il comandamento "non uccidere" quando "un venti per cento della popolazione mondiale consuma risorse in misura tale da rubare alle nazioni povere e alle future generazioni ciò di cui hanno bisogno per sopravvivere"»(Ls 95). La settima ed ultima pista di riflessione invita a porre lo sguardo su Gesù per mezzo del quale tutte le cose sono state create (Col 1,16; Gv 1,1-18). Egli a più riprese ha ricordato che il Padre ama tutte le creature (Lc 12,6; Mt 6,26), ha invitato a contemplare la natura $(\mathrm{Gv} 4,35)$ di cui si è dimostrato Signore $(\mathrm{Mt}$ 8,27 ) e non ha mai sostenuto le «filosofie che disprezzavano il corpo, la materia e le realtà di questo mondo» (Ls 98). Egli inoltre «lavorava con le sue mani, prendendo contatto quotidiano con la materia creata da Dio per darle forma con la sua abilità di artigiano» (Ls 98). È ancora più notevole costatare che «il Nuovo Testamento non solo ci parla del Gesù terreno e della sua relazione tanto concreta e amorevole con il mondo. Lo mostra anche risorto e glorioso, presente 
in tutto il creato con la sua signoria universale». Questa consapevolezza «ci proietta alla fine dei tempi, quando il Figlio consegnerà al Padre tutte le cose, così che "Dio sia tutto in tutti" (1Cor 15,28). In tal modo, le creature di questo mondo non ci si presentano più come una realtà meramente naturale, perché il Risorto le avvolge misteriosamente e le orienta a un destino di pienezza». (Ls 100).

\section{La radice umana della crisi ecologica}

Il terzo capitolo inizia sostenendo: «A nulla servirà descrivere $\mathrm{i}$ sintomi, se non riconosciamo la radice umana della crisi ecologica» (Ls 101). Tale crisi è causata dalla tendenza diffusa ad accettare acriticamente lo sviluppo tecnologico. Questo procedere pone l'umanità di fronte ad un bivio. Se da un lato, infatti, non si può non apprezzare gli enormi benefici che la tecnica ha prodotto per il miglioramento del livello della qualità della vita, dall'altro canto risulta evidente che tale incremento può diventare la fonte di un pericoloso dominio.

Sono, infatti, segno di un notevole beneficio per l'umanità le nuove possibilità date dal potenziamento della medicina, dell'ingegneria e delle comunicazioni. È altrettanto importante l'elaborazione di alternative valide per favorire lo sviluppo sostenibile e l'impegno ad incrementare tutto ciò che favorisce la ricerca della bellezza nelle arti e nella cultura.

Non si può però dimenticare che l'energia nucleare, le biotecnologie, l'informatica, la genetica, messe nelle mani di un potere economico-politico senza scrupoli, possono diventare fonte di innumerevoli problemi. Basti ricordare a conferma «le bombe atomiche lanciate in pieno xx secolo, come il grande spiegamento di tecnologia ostentato dal nazismo, dal comunismo e da altri regimi totalitari al servizio dello sterminio di milioni di persone» (Ls 104).

Di fronte a questi rischi, non si può continuare a sostenere che ogni nuova acquisizione tecnologica costituisca senza riserve una fonte di progresso. Molti continuano a pensare così solo perché non sono stati educati al «retto uso della potenza» (Ls 105). È evidente che «l'immensa crescita tecnologica non è stata accompagnata da uno sviluppo dell'essere umano per quanto riguarda la responsabilità, i valori e la coscienza [...] dei propri limiti» (Ls 105) con il conseguente 
«rischio di faticare a comprendere «la serietà delle sfide che si presentano» (Ls 105).

Questa difficoltà, infatti, non è pienamente percepita quando la libertà si consegna «alle forze cieche dell'inconscio, dei bisogni immediati, dell'egoismo, della violenza brutale» (Ls 105). Quando ciò accade, l'uomo si trova «nudo ed esposto di fronte al suo stesso potere che continua a crescere, senza avere gli strumenti per controllarlo. Può disporre di meccanismi superficiali, ma possiamo affermare che gli mancano un'etica adeguatamente solida, una cultura e una spiritualità che realmente gli diano un limite e lo contengano entro un lucido dominio di sé» (Ls 105).

Il Papa sviluppa questo suo pensiero nelle due parti successive del terzo capitolo. Nella prima (Ls 106-114) riflette sulla globalizzazione del paradigma tecnocratico. Nota che è particolarmente problematico il modo ingenuo con il quale l'umanità ha assunto la tecnologia. Da sempre - continua il Papa - l'uomo è intervenuto sulla natura. Nei tempi passati però ha agito con lo scopo «di accompagnare, di assecondare le possibilità offerte dalle cose stesse» (Ls 106). Ora, invece, «ciò che interessa è estrarre tutto ciò che è possibile dalle cose attraverso l'imposizione della mano umana» (Ls 106).

Alla base di questo procedere c'è l'irrealistica opinione che si debba disporre in modo illimitato dei beni limitati della terra (LS 106). Tale ingenuità ha determinato il fatto che «l'essere umano e le cose hanno cessato di darsi amichevolmente la mano diventando dei contendenti» (Ls 106). Le conseguenze sono notevoli. Assistiamo, infatti, al continuo degrado dell'ambiente, al condizionamento degli stili di vita, al dominio di gruppi di potere capaci di influenzare le scelte economiche e politiche, al consumismo sfrenato occidentale, contrapposto all'estrema povertà dei paesi del Terzo e Quarto Mondo, all'emarginazione della filosofia e dell'etica dalle scelte del vivere sociale (Ls 107-110).

È necessario correre al più presto ai ripari con una sana cultura ecologica che non si accontenti di rispondere alle emergenze ambientali, ma che proponga un diverso pensiero politico, un più accorto programma educativo, un nuovo stile di vita capace di favorire «un altro tipo di progresso, più sano, più umano, più sociale e più integrale» (Ls 112).

Questa nuova sensibilità si attua già - ricorda il $\mathrm{Pa}-$ pa - «quando comunità di piccoli produttori optano per 
sistemi di produzione meno inquinanti [...]. O quando la tecnica si orienta prioritariamente a risolvere i problemi concreti degli altri [...]. E ancora quando la ricerca creatrice del bello e la sua contemplazione riescono a superare il potere oggettivante in una sorta di salvezza che si realizza nel bello e nella persona che lo contempla» (Ls 112).

Affinché questa mentalità si diffonda, è necessario proporre una «coraggiosa rivoluzione culturale» che non significa «tornare all'epoca delle caverne» ma «rallentare la marcia per guardare la realtà in un altro modo, raccogliere gli sviluppi positivi e sostenibili, e al tempo stesso recuperare i valori e i grandi fini distrutti da una sfrenatezza megalomane» (Ls 114).

Nell'ultima parte del capitolo il Papa evidenzia che oggi si sta verificando un eccesso di antropocentrismo che rende autoreferenziali, centrati unicamente sul proprio sé e sul proprio potere (Ls 116). Ne deriva una logica dell'«usa e getta» che "produce tanti rifiuti solo per il desiderio disordinato di consumare più di quello di cui realmente si ha bisogno» (Ls 123). In modo ancor più allarmante, lo scarto «spinge una persona ad approfittare di un'altra e a trattarla come un mero oggetto, obbligandola a lavori forzati, o riducendola in schiavitù a causa di un debito. È la stessa logica che porta a sfruttare sessualmente i bambini, o ad abbandonare gli anziani che non servono ai propri interessi». È una logica che ha dei risvolti anche economici perché induce ad accettare che forze invisibili del mercato abbiano il dominio. Quando, infatti, «non ci sono verità oggettive né principi stabili $[\ldots]$ che limiti possono avere la tratta degli esseri umani, la criminalità organizzata, il narcotraffico, il commercio di diamanti insanguinati e di pelli di animali in via di estinzione?». Si insinua così una logica relativista che «giustifica l'acquisto di organi dei poveri allo scopo di venderli o di utilizzarli per la sperimentazione, o lo scarto di bambini perché non rispondono al desiderio dei loro genitori». È la logica «"usa e getta” che produce tanti rifiuti solo per il desiderio disordinato di consumare più di quello di cui realmente si ha bisogno». In questa prospettiva «non possiamo pensare che i programmi politici o la forza della legge basteranno ad evitare i comportamenti che colpiscono l'ambiente, perché quando è la cultura che si corrompe e non si riconosce più alcuna verità oggettiva o principi 
universalmente validi, le leggi verranno intese solo come imposizioni arbitrarie e come ostacoli da evitare» (Ls 123).

Subito dopo sono affrontate due questioni cruciali. La prima riguarda il lavoro. Il Papa sostiene: "Non si deve cercare di sostituire sempre più il lavoro umano con il progresso tecnologico: così facendo l'umanità danneggerebbe sé stessa. Il lavoro è una necessità, è parte del senso della vita su questa terra, via di maturazione, di sviluppo umano e di realizzazione personale». Conseguentemente «aiutare i poveri con il denaro dev'essere sempre un rimedio provvisorio per fare fronte a delle emergenze. Il vero obiettivo dovrebbe sempre essere di consentire loro una vita degna mediante il lavoro». Invece «l'economia ha favorito un tipo di progresso tecnologico finalizzato a ridurre i costi di produzione in ragione della diminuzione dei posti di lavoro, che vengono sostituiti dalle macchine». Va ribadito che «la riduzione dei posti di lavoro "ha anche un impatto negativo sul piano economico, attraverso la progressiva erosione del 'capitale sociale', ossia di quell'insieme di relazioni di fiducia, di affidabilità, di rispetto delle regole, indispensabili ad ogni convivenza civile"». Se ne può dedurre che «"i costi umani sono sempre anche costi economici e le disfunzioni economiche comportano sempre anche costi umani”. Rinunciare ad investire sulle persone per ottenere un maggior profitto immediato è un pessimo affare per la società» (Ls 128).

L'ultima parte del capitolo è dedicata essenzialmente a riflettere sui vantaggi e sui limiti determinati dall'uso degli OGM (Ls 132-136). Il Papa precisa che è «una questione di carattere complesso» (par. 135) per cui è particolarmente difficile «dare un giudizio adeguato»(Ls 133). In alcune regioni, infatti, «il loro utilizzo ha prodotto una crescita economica che ha contribuito a risolvere alcuni problemi» (Ls 134). Sussistono peraltro significative difficoltà «che non devono essere minimizzate» (Ls 134). Prima tra tutte, la «concentrazione di terre produttive nelle mani di pochi» (Ls 134). Permane poi il dubbio che possano provocare danni alla salute dell'uomo. Per questo auspica che si favorisca «un dibattito scientifico e sociale che sia responsabile e ampio, in grado di considerare tutta l'informazione disponibile e di chiamare le cose con il loro nome» a partire da «linee di ricerca autonoma e interdisciplinare»(Ls 135). 
Il quarto capitolo dell'enciclica presenta dal titolo il tema dell'ecologia integrale. Questa espressione ricorre per ben altre nove volte e rappresenta la prospettiva focale su cui si regge tutto il documento nella convinzione che non possiamo "considerare la natura come qualcosa di separato da noi o come una mera cornice della nostra vita» (Ls 139).

In un articolo di Giacomo Costa e Paolo Foglizzo, si legge a proposito:

Papa Francesco assume il termine "ecologia" non nel significato generico e spesso superficiale di una qualche preoccupazione "verde", ma in quello ben più profondo di approccio a tutti i sistemi complessi la cui comprensione richiede di mettere in primo piano la relazione delle singole parti tra loro e con il tutto. Il riferimento è all'immagine di ecosistema. L'ecologia integrale diventa così il paradigma capace di tenere insieme fenomeni e problemi ambientali (riscaldamento globale, inquinamento, esaurimento delle risorse, deforestazione, ecc.) con questioni che normalmente non sono associate all'agenda ecologica in senso stretto, come la vivibilità e la bellezza degli spazi urbani o il sovraffollamento dei trasporti pubblici. Ancora di più, l'attenzione ai legami e alle relazioni consente di utilizzare l'ecologia integrale anche per leggere il rapporto con il proprio corpo (Ls 155), o le dinamiche sociali e istituzionali a tutti i livelli (Ls 142). Si può quindi parlare di una dimensione sociale dell'ecologia, o meglio di una vera e propria "ecologia sociale". ${ }^{13}$

Questa prospettiva nasce dalla convinzione che le sorti dell'umanità e dell'ambiente sono intimamente legate. Già il San Paolo VI, nella Octogesima Adveniens pubblicata il 14 maggio 1971 aveva osservato:

Mentre l'orizzonte dell'uomo si modifica, un'altra trasformazione si avverte, conseguenza tanto drammatica dell'attività umana. L'uomo ne prende coscienza bruscamente: attraverso uno sfruttamento sconsiderato della natura, egli rischia di distruggerla e di essere a sua volta vittima di siffatta degradazione. Non soltanto l'ambiente materiale diventa una minaccia permanente: inquinamenti e rifiuti, nuove malattie, potere distruttivo totale; ma è il contesto 
umano, che l'uomo non padroneggia più, creandosi così per il domani un ambiente che potrà essergli intollerabile: problema sociale di vaste dimensioni che riguarda l'intera famiglia umana. A queste nuove prospettive il cristiano deve dedicare la sua attenzione, per assumere, insieme con gli altri uomini, la responsabilità di un destino diventato ormai comune. ${ }^{14}$

Il primo giugno 1972, nel messaggio inviato in occasione dell'inaugurazione della Conferenza delle Nazioni Unite sull'ambiente, aveva aggiunto: "Tutte le misure tecniche rimangono inefficaci se non vengono applicate con la presa di coscienza della necessità di un cambiamento radicale della mentalità $[. .$.$] secondo le esigenze di una vera etica$ [...] che si basa principalmente sul rapporto tra l'uomo e il suo ambiente» ${ }^{15}$.

San Giovanni Paolo II ha ripreso questo tema in diversi interventi ed in particolare nella Sollicitudo rei socialis (1987) e nella Centesimus annus (1991). Egli ha evidenziato che la questione ecologica ha un profondo legame con la crisi morale che l'umanità attraversa e ha espressamente parlato di "ecologia naturale", "ecologia umana" e "ecologia sociale". Particolarmente indicativo è al riguardo il paragrafo 34 della Sollicitudo rei socialis dove si legge

Il dominio accordato dal Creatore all'uomo non è un potere assoluto, né si può parlare di libertà di "usare e abusare", o di disporre delle cose come meglio aggrada. La limitazione imposta dallo stesso Creatore fin dal principio, ed espressa simbolicamente con la proibizione di "mangiare il frutto dell'albero" (Gen2,16), mostra con sufficiente chiarezza che, nei confronti della natura visibile, siamo sottomessi a leggi non solo biologiche, ma anche morali, che non si possono impunemente trasgredire. ${ }^{16}$

Altrettanto interessante è stato il suo Messaggio per la giornata mondiale della pace del 1990. Nei primi due paragrafi sostenne:

14. Paolo VI, Lett. Ap., Octogesima adveniens (14 maggio 1971), AAS 63(1971), 401-441: 21.

15. Id., «Messaggio in occasione dell'inaugurazione della Conferenza delle Nazioni Unite sull'ambiente», in Insegnamenti X/1972, 608.

16. Giovanni Paolo II, Lett. Enc. Sollicitudo rei socialis (30 dicembre 1987): AAS 80(1988), 1653-1729: 34. 
Si avverte ai nostri giorni la crescente consapevolezza che la pace mondiale sia minacciata [...] anche dalla mancanza del dovuto rispetto per la natura, dal disordinato sfruttamento delle sue risorse e dal progressivo deterioramento della qualità della vita [...]. Di fronte al diffuso degrado ambientale l'umanità si rende ormai conto che non si può continuare ad usare i beni della terra come nel passato [...]. Sta così formandosi una coscienza ecologica, che non deve essere mortificata, ma anzi favorita, in modo che si sviluppi e maturi trovando adeguata espressione in programmi ed iniziative concrete. Non pochi valori etici, di fondamentale importanza per lo sviluppo di una società pacifica, hanno una diretta relazione con la questione ambientale. ${ }^{17}$

Benedetto XVI ha fatto proprie le istanze del predecessore. Nella Caritas in veritate ha ribadito che la questione ecologica riguarda i cristiani proprio in quanto persone di fede: «La Chiesa ha una responsabilità per il creato e deve far valere questa responsabilità anche in pubblico. E facendolo deve difendere la terra, l'acqua e l'aria come doni della creazione, appartenenti a tutti» ${ }^{18}$. Nell'Udienza del 26 agosto 2009 ha aggiunto: «la protezione dell'ambiente, la tutela delle risorse e del clima richiedono che i responsabili internazionali agiscano congiuntamente nel rispetto della legge e della solidarietà, soprattutto nei confronti delle regioni più deboli della terra» ${ }^{19}$.

Alla luce di questi precedenti pronunciamenti, Papa Francesco inizia il quarto capitolo precisando: «Dal momento che tutto è intimamente relazionato e che gli attuali problemi richiedono uno sguardo che tenga conto di tutti gli aspetti della crisi mondiale, propongo di soffermarci adesso a riflet-

17. Id., «Messaggio per la giornata mondiale della pace», 1990, in w.vatican.va/content/ john-paul-ii/it/messages/peace/documents/hf_jp-ii_mes_19891208_xxiii-world-day-forpeace.html [16 marzo 2020]. Cfr. anche: Giovanni Paolo II, Centesimus annus, 1991: 3738; Evangelium vitae, 1995: 10.27.42; Messaggio per la giornata mondiale della pace (1999); Catechismo della Chiesa Cattolica, 1992: 2415; Compendio della Dottrina Sociale della Chiesa, 2004: 451-487.

18. Benedetto XVI, Lett. Enc. Caritas in veritate (29 giugno 2009): AAS 101(2009), 641709: 51 .

19. Id., Udienza Generale (26 agosto 2009), in http://www.vatican.va/content/benedictxvi/it/audiences/2009/documents/hf_ben-xvi_aud_20090826.html [16 marzo 2020]. Cfr. anche: Benedetto XVI, Messaggio per la giornata mondiale della pace (2007 e 2010), Messaggio per la giornata mondiale del turismo (2008). 
tere sui diversi elementi di una ecologia integrale, che comprenda chiaramente le dimensioni umane e sociali» (Ls 137).

Individua subito tre ambiti di riflessione. Il primo riguarda il rapporto che deve intercorrere tra cura dell'ambiente, gestione economica e vita sociale. Il Papa rileva la necessità di «cercare soluzioni integrali, che considerino le interazioni dei sistemi naturali tra loro e con i sistemi sociali. Non ci sono due crisi separate, una ambientale e un'altra sociale, bensì una sola e complessa crisi socio-ambientale. Le direttrici per la soluzione richiedono un approccio integrale per combattere la povertà, per restituire la dignità agli esclusi e nello stesso tempo per prendersi cura della natura» (LS 139). Ne deriva che «se tutto è in relazione, anche lo stato di salute delle istituzioni di una società comporta conseguenze per l'ambiente e per la qualità della vita umana: "Ogni lesione della solidarietà e dell'amicizia civica provoca danni ambientali"»(Ls 142). Ad esempio «il consumo di droghe nelle società opulente provoca una costante o crescente domanda di prodotti che provengono da regioni impoverite, dove si corrompono i comportamenti, si distruggono vite e si finisce col degradare l'ambiente» (Ls 142).

A proposito dell'ecologia culturale, segnala che «l'ecologia richiede anche la cura delle ricchezze culturali dell'umanità nel loro significato più ampio». Si tratta «di prestare attenzione alle culture locali nel momento in cui si analizzano questioni legate all'ambiente, facendo dialogare il linguaggio tecnico-scientifico con il linguaggio popolare». In questo modo la cultura non è solo intesa come un monumento del passato, ma in senso vivo, dinamico e partecipativo (par. 143). Trattando poi del quotidiano, invita a produrre un miglioramento integrale della vita umana attraverso la cura delle strutture abitative, degli spazi pubblici, dei servizi urbani e rurali perché «gli ambienti in cui viviamo influiscono sul nostro modo di vedere la vita, di sentire e di agire» (Ls 147).

A conclusione del capitolo, propone di valorizzare il principio del bene comune, indispensabile per un'armonica vita civile (Ls 156). Citando l'insegnamento di Gaudium et spes 26, ricorda che il bene comune è «'insieme di quelle condizioni della vita sociale che permettono tanto ai gruppi quanto ai singoli membri di raggiungere la propria perfezione più pienamente e più speditamente». Nota che oggi «si riscon- 
trano tante iniquità e sono sempre più numerose le persone che vengono scartate, private dei diritti umani fondamentali» (Ls 158). Precisa che impegnarsi per il bene comune significa prima di tutto porre l'opzione preferenziale per i poveri (Ls 158). Inoltre, invita a pensare anche alle generazioni che verranno perché è iniquo «lasciare alle prossime generazioni troppe macerie, deserti e sporcizia» (Ls 161). Evidenzia che «il ritmo di consumo, di spreco e di alterazione dell'ambiente ha superato le possibilità del pianeta, in maniera tale che lo stile di vita attuale, essendo insostenibile, può sfociare solamente in catastrofi, come di fatto sta già avvenendo periodicamente in diverse regioni»(Ls 161). Non è possibile continuare in questa logica perversa perché solo ponendo ora un freno e possibile attenuare gli effetti nefasti degli squilibri ambientali. Per realizzare questo progetto, bisogna contrastare l'individualismo e i tanti problemi sociali derivati dalla «ricerca egoistica della soddisfazione immediata, con le crisi dei legami familiari e sociali, con le difficoltà a riconoscere l'altro». Bisogna far fronte ai consumi eccessivi che danneggiano le giovani generazioni «che trovano sempre più difficoltà ad acquistare una casa propria e a fondare una famiglia. Inoltre, questa incapacità di pensare seriamente alle future generazioni è legata alla nostra incapacità di ampliare l'orizzonte delle nostre preoccupazioni e pensare a quanti rimangono esclusi dallo sviluppo»(Ls 162).

\section{Alcune linee di orientamento e di azione}

Il capitolo quinto offre alcune linee di orientamento e di azione nella consapevolezza che non è sufficiente individuare gli stili di vita dannosi, ma è indispensabile anche tracciare percorsi di dialogo condivisi che «aiutino ad uscire dalla spirale di autodistruzione in cui stiamo affondando» (Ls 163). Papa Francesco nota che negli ultimi decenni le questioni ambientali hanno dato origine a un ampio dibattito che ha coinvolto anche istituzioni internazionali (Ls 165). Particolarmente rilevanti sono stati il vertice mondiale sull'ambiente che si è tenuto a Rio de Janeiro nel 1992, la stesura nel 1989 della convenzione di Basilea sui rifiuti pericolosi, la Conferenza delle Nazioni Unite tenuta nel 2012 e denominata Rio +20. Ciononostante, il lavoro da compiere è ancora notevole anche perché, per mancanza di sufficiente decisione politica, permangono gravi questioni ambientali irrisolte quali le 
emissioni di gas inquinanti, la governance degli oceani e il riscaldamento globale (Ls 175). È necessaria pertanto una diversificata strategia di superamento dell'attuale impasse. Il Papa indica al riguardo alcune priorità.

1. Nuove politiche nazionali e locali (Ls 176-181): spiega che «la società, attraverso organismi non governativi deve obbligare i governi a sviluppare normative, procedure e controlli più rigorosi. Se i cittadini non controllano il potere politico - nazionale, regionale e municipale - neppure è possibile un contrasto dei danni ambientali» (Ls 179).

2. Maggiore trasparenza nei processi decisionali (Ls 182-188). Ogni iniziativa imprenditoriale dovrebbe essere attuata solo dopo aver condotto un attento discernimento guidato da alcune essenziali domande: «Per quale scopo? Per quale motivo? Dove? Quando? In che modo? A chi è diretto? Quali sono i rischi? A quale costo? Chi paga le spese e come lo farà?»(Ls 185).

3. Disponibilità al dialogo tra economia e politica (Ls 189198). È necessario «evitare una concezione magica del mercato, che tende a pensare che i problemi si risolvano solo con la crescita dei profitti delle imprese o degli individui» (Ls 190). Abbiamo bisogno - continua il Papa - di una politica che pensi con una visione ampia, e che porti avanti un nuovo approccio integrale, includendo, in un dialogo interdisciplinare, i diversi aspetti della crisi» (Ls 198).

4. Dialogo delle religioni con le scienze (Ls 199-201). Il Papa al riguardo osserva: "La maggior parte degli abitanti del pianeta si dichiarano credenti, e questo dovrebbe spingere le religioni ad entrare in un dialogo tra loro orientato alla cura della natura, alla difesa dei poveri, alla costruzione di una rete di rispetto e di fraternità». È inoltre «indispensabile un dialogo tra le stesse scienze, dato che ognuna è solita chiudersi nei limiti del proprio linguaggio, e la specializzazione tende a diventare isolamento e assolutizzazione del proprio sapere. Questo impedisce di affrontare in modo adeguato i problemi dell'ambiente». Infine, «si rende necessario un dialogo aperto e rispettoso tra i diversi movimenti ecologisti, fra i quali non mancano le lotte ideologiche. La gravità della crisi ecologica esige da noi tutti di pensare al bene comune e di andare avanti sulla via del dialogo che richiede pazienza, ascesi e generosità, ricordando sempre che "la realtà è superiore all'idea"» (Ls 201). 
Il sesto e ultimo capitolo è molto ricco di spunti pratici per andare oltre al degrado contemporaneo e «ritornare a scegliere il bene e rigenerarsi, al di là di qualsiasi condizionamento psicologico e sociale» (LS 205). Sono necessari - precisa il Papa - nuove convinzioni, nuovi atteggiamenti e nuovi stili di vita atti a promuovere processi di rigenerazione culturale, spirituale ed educativa (Ls 202). L'enciclica propone al riguardo alcuni ineliminabili passaggi.

1. Educare all'alleanza tra l'umanità e l'ambiente (Ls 209215). Si deve creare una "cittadinanza ecologica" formata da diversi agenti educativi: scuola, famiglia, mezzi di comunicazione, catechesi, politica e associazioni (Ls 213-214). In questo contesto - attesta il documento - non va trascurata la relazione che sussiste tra un'adeguata educazione al senso del bello e alla cura di un ambiente sano per uscire dal pragmatismo utilitaristico. "Allo stesso tempo, se si vuole raggiungere dei cambiamenti profondi, bisogna tener presente che i modelli di pensiero influiscono realmente sui comportamenti». Infatti, «l'educazione sarà inefficace e i suoi sforzi saranno sterili se non si preoccupa anche di diffondere un nuovo modello riguardo all'essere umano, alla vita, alla società e alla relazione con la natura. Altrimenti continuerà ad andare avanti il modello consumistico trasmesso dai mezzi di comunicazione e attraverso gli efficaci meccanismi del mercato» (Ls 215).

2. Promuovere una conversione ecologica (Ls 216-221).Per alimentare la passione per la cura del creato i cristiani devono «lasciar emergere tutte le conseguenze dell'incontro con Gesù nella relazione con il mondo che li circonda» (LS 217). Papa Francesco propone una conversione personale (Ls 218) che stimoli a «riconoscere i propri errori, peccati, vizi o negligenze» e una conversione comunitaria (Ls 219221) che unisca le forze per riscoprire il mondo come dono ricevuto, non dimenticare le altre creature, mettere a servizio le capacità di ciascuno, riconoscere l'ordine e il dinamismo inserito da Dio nel mondo e agire in corrispondenza.

3. Vivere nella gioia e nella pace (Ls 222-227). Il documento osserva «il costante cumulo di possibilità di consumare distrae il cuore e impedisce di apprezzare ogni cosa e ogni momento». Si può uscire da questa logica perversa renden- 
dosi «presenti serenamente davanti ad ogni realtà». In questo modo si evidenziano «molte più possibilità di comprensione e di realizzazione personale». La spiritualità cristiana propone così il «ritorno alla semplicità che ci permette di fermarci a gustare le piccole cose, di ringraziare delle possibilità che offre la vita senza attaccarci a ciò che abbiamo né rattristarci per ciò che non possediamo. Questo richiede di evitare la dinamica del dominio e della mera accumulazione di piaceri» (Ls 222). Il testo aggiunge che la sobrietà è liberante (Ls 224), umile (Ls 224), porta pace interiore (Ls 225), apre alla lode e al ringraziamento (Ls 227).

4. Crescere nell'amore civile e politico (Ls 228-232). L'amore per la natura - spiega - non può essere disgiunto dall'amore per gli altri perché «occorre sentire nuovamente che abbiamo bisogno gli uni degli altri, che abbiamo una responsabilità verso gli altri e verso il mondo, che vale la pena di essere buoni e onesti». Purtroppo, siamo stati già troppo a lungo «nel degrado morale, prendendoci gioco dell'etica, della bontà, della fede, dell'onestà, ed è arrivato il momento di riconoscere che questa allegra superficialità ci è servita a poco». È la «distruzione di ogni fondamento della vita sociale finisce col metterci l'uno contro l'altro per difendere i propri interessi, provoca il sorgere di nuove forme di violenza e crudeltà e impedisce lo sviluppo di una vera cultura della cura dell'ambiente» (Ls 229). E invece possibile ripercorrere la piccola via dell'amore proposta da santa Teresa di Lisieux perché «un'ecologia integrale è fatta anche di semplici gesti quotidiani nei quali spezziamo la logica della violenza, dello sfruttamento, dell'egoismo» (Ls 230).

5. Considerare i segni sacramentali e accogliere l'invito al riposo celebrativo (Ls 233-237). Non si deve «passare dall'esteriorità all'interiorità per scoprire l'azione di Dio nell'anima» (Ls 233) perché, come insegnava San Giovanni della Croce, «tutto quanto c'è di buono nelle cose e nelle esperienze del mondo "si trova intimamente unito in Dio in maniera infinita” (Ls 234). I sacramenti stessi sono il luogo privilegiato in cui «la natura viene assunta da Dio e trasformata in mediazione della vita soprannaturale [...]. Lacqua, l'olio, il fuoco e i colori sono assunti con tutta la loro forza simbolica e si incorporano nella lode»(Ls 234). In particolare, nell'Eucaristia «il creato trova la sua maggiore elevazione» 
perché in essa è «già realizzata la pienezza, ed è il centro vitale dell'universo, il centro traboccante di amore e di vita inesauribile». Cosicché «unito al Figlio incarnato, presente nell'Eucaristia, tutto il cosmo rende grazie a Dio». Infatti, «l'Eucaristia è di per sé un atto di amore cosmico: "Sì, cosmico! Perché anche quando viene celebrata sul piccolo altare di una chiesa di campagna, l'Eucaristia è sempre celebrata, in certo senso, sull'altare del mondo". L'Eucaristia unisce il cielo e la terra, abbraccia e penetra tutto il creato» (Ls 236).

6. Aprirsi alla lode per la Trinità (Ls 238-240). Credere in un Dio unico per i cristiani significa, inoltre, riconoscere che «tutta la realtà contiene in sé un'impronta propriamente trinitaria» (Ls 239).

7. Guardare a Maria, regina del creato perché «nel suo corpo glorificato, insieme a Cristo risorto, parte della creazione ha raggiunto tutta la pienezza della sua bellezza» e a San Giuseppe, custode della Chiesa universale, che «può motivarci a lavorare con generosità e tenerezza per proteggere questo mondo che Dio ci ha affidato»(Ls 241).

8. Operare nell'attesa dell'incontro definitivo con il Creatore (LS 243-246). L'enciclica termina con una considerazione sul compimento ultimo che ci farà incontrare «faccia a faccia con l'infinita bellezza di Dio (cfr. 1 Cor 13,12)» e farci «leggere con gioiosa ammirazione il mistero dell'universo, che parteciperà insieme a noi della pienezza senza fine» (Ls 243). Perché questo si realizzi, è urgente farsi «carico di questa casa che ci è stata affidata, sapendo che ciò che di buono vi è in essa verrà assunto nella festa del cielo» (Ls 244).

\section{Conclusione}

Il monito del Papa invita credenti e non credenti ad un serio esame di coscienza che porti ad una autentica conversione ecologica integrale. $\mathrm{Ne}$ va del destino dell'umanità. È indispensabile, infatti, che tutti sentano il dovere di cooperare alla salvaguardia dell'ecosistema e allo sviluppo integrale dell'umanità con una particolare attenzione alle popolazioni più disagiate. Solo così potremo vivere in un modo più giusto e ospitale per noi e per le nuove generazioni. 


\section{Bibliografia}

Benedetto XVI. 2009. Lett. Enc. Caritas in veritate (29 giugno 2009): $A A S$ 101, 641-709.

- Udienza Generale (26 agosto 2009), in http://www.vatican.va/ content/benedict-xvi/it/audiences/2009/documents/hf_benxvi_aud_20090826.html.

Blondet M. 1994. Hitler, un si all'aborto che precorre Il Cairo, "Avvenire», 14 agosto.

Costa G., Foglizzo P. 2015. Lecologia integrale, «Aggiornamenti Sociali», 8-9.

Deaton A. 2015. La grande fuga, il Mulino, Bologna.

Ellis E.C. 2013. Overpopulation Is Not the Problem, «New York Times», 13 settembre.

Francesco. 2014. "Discorso in occasione alla II Conferenza Internazionale sulla nutrizione», Sede della Fao, Roma 24 novembre 2014, in http:/www.vatican.va/content/francesco/it/speeches/2014/november/documents/papa-francesco_20141120_visita-fao.Ht ml.

- 2013. «Omelia della Messa d'inizio pontificato», 19 Marzo 2013, inhttp://www.vatican.va/content/francesco/it/homilies/2013/documents/papa-francesco_20130319_omelia-inizio-pontificato.html.

- 2013. Lett. enc. Lumen fidei (29 giugno 2013): AAS 105, 555596.

Giovanni Paolo II. 1990. «Messaggio per la giornata mondiale della pace», in w.vatican.va/content/john-paul-ii/it/messages/ peace/documents/hf_jp-ii_mes_19891208_xxiii-world-dayfor-peace.html.

- 1988. Lett. enc. Sollicitudo rei socialis (30 dicembre 1987): AAS 80, 1653-1729.

Malaspina F. 2008. I limiti del sottosviluppo, «Zenit», 8 luglio.

Paolo VI. 1972. "Messaggio in occasione dell'inaugurazione della Conferenza delle Nazioni Unite sull'ambiente», in Insegnamenti X.

- 1971. Lett. Ap., Octogesima adveniens(14 maggio 1971), AAS 63, 401-441.

Prince Philip. 1988. riportato da Deuteche Press Agentur, August.

Sartori G. 2013. Una modernità fuori misura, «Corriere della Sera», 25 novembre.

Veronesi U. 2014. Un mondo senza fame? Con la scienza si può, «Oggi», 26 settembre.

Watson P.J. 2006. Top scientist advocates mass culling of 90\% human population, «PrisonPlanet», 3 aprile. 



\section{Comunità umane solidali e inclusive (obiettivi 4, 10, 11 e 16 Agenda Onu 2030)}

aA 

II.1. Gli obiettivi dello sviluppo sostenibile

aA 


sostenibile», definendolo «uno sviluppo che soddisfa i bisogni del presente senza compromettere la possibilità delle generazioni future di soddisfare i propri». Ben si può affermare come la dimensione originaria del concetto sia piuttosto circoscritta e sostanzialmente limitata alla prospettiva ecologica, e, più precisamente, al risparmio delle materie prime e, in generale, delle risorse ambientali ${ }^{1}$.

Risale tuttavia a qualche anno più tardi e, precisamente, alla Conferenza di Rio de Janeiro del 1992, United $\mathrm{Na}$ tions Conference on Environment and Development: UNCED or Earth Summit, il vero e proprio conio dell'espressione «sviluppo sostenibile», come «diritto allo sviluppo» da attuarsi «in modo da soddisfare equamente i bisogni di sviluppo e ambientali delle generazioni presenti e future»; con tale 
dichiarazione, l'attenzione si concentra in modo più specifico sulla relazione tra ambiente e sviluppo, consolidando la prospettiva di cooperazione, condivisione e di consenso internazionale (principio 12 e sgg.); prendono forma, così, i cd. tre pilastri dello sviluppo sostenibile, ossia quello economico, quello di tutela ambientale e quello sociale, sulla constatazione per cui un diritto ambientale «efficace», non può ripercuotersi negativamente, dal punto di vista dei costi economici e sociali, sui Paesi terzi, in particolare su quelli in via di sviluppo (principio 11).

Molta strada è stata fatta da allora, ma sostanzialmente l'impostazione sui tre pilastri emersa da quella Conferenza si è consolidata nel tempo e, pur con progressivi arricchimenti e puntualizzazioni, si è confermata come struttura fondamentale del principio stesso.

Il 25 settembre 2015 è stato adottato, a New York, il documento finale del Summit delle Nazioni Unite dedicato all'adozione di un programma di sviluppo per il periodo successivo al 2015, intitolato appunto «Transforming our world: the 2030 Agenda for Sustainable Development». L'Agenda 2030 per lo sviluppo sostenibile rappresenta ad oggi l'approdo più recente del tema e plasma l'idea più matura di «sviluppo sostenibile».

Il programma che ne emerge è pervaso dall'idea di collaborazione, evidente fin dal Preambolo in cui si afferma come «tutti i Paesi e tutte le parti in causa, agendo in associazione collaborativa, implementeranno questo programma», e rappresenta un piano d'azione per l'umanità e il pianeta diretto a tutti i Paesi del Mondo, senza distinzioni quelli sviluppati e quelli in via di sviluppo, tutti chiamati ad agire per metterlo in opera, di concerto e ciascuno secondo le proprie capacità.

Secondo una linea di continuità coi Summit che l'hanno preceduto, a partire da quello di Rio del Janeiro del 1992, esso poggia sui tre pilastri dello sviluppo sostenibile, ossia, come si è detto, lo sviluppo economico, l'inclusione sociale e la tutela dell'ambiente, di cui si riafferma solennemente la stretta interdipendenza.

Rispetto ai precedenti, tuttavia, l'Agenda 2030 ha l'espressa ambizione di costituire un accordo storico portatore di una serie completa di obiettivi (e targets) di carattere universale che si iscrivono nel prolungamento degli obiettivi 
del Millennio per lo sviluppo, considerati irrinunciabili per la trasformazione generale finalizzata a liberare l'umanità dalla povertà e dal bisogno di prendersi cura del pianeta e di preservarlo. Il programma, tuttavia, va molto al di là degli obiettivi del Millennio: da una parte, infatti, si attribuisce particolare attenzione a obiettivi che, anche se inclusi tra quelli, non erano stati raggiunti (in particolare quelli relativi alla salute materna, neonatale e infantile e alla salute riproduttiva); d'altra parte, e per quanto qui di interesse, esso pone anche molti nuovi obiettivi economici, sociali e ambientali, e prevede altresì esplicitamente l'avvento di società più pacifiche e inclusive.

Ai fini che qui rilevano, si può osservare come i 17 obiettivi del programma - e i relativi 169 nuovi targets - concernano problematiche molto ampie e complesse : eliminazione della povertà e della fame; buona salute e benessere; educazione di qualità; acqua pulita e igiene; energia pulita a costi abbordabili; lavoro dignitoso e crescita economica; industria, innovazione e infrastrutture; riduzione delle disuguaglianze; città e comunità sostenibili; consumi responsabili; lotta contro il cambiamento climatico; vita sott'acqua e sulla terra, pace e giustizia e istituzioni forti, partnership per la realizzazione degli obiettivi.

Di particolare interesse in questa sede, l' $11^{\circ}$ obiettivo, intitolato a «città e insediamenti umani sostenibili», che comprende l'impegno a «rendere le città e gli insediamenti umani inclusivi, sicuri, resilienti e sostenibili».

Lattenzione per le città e, in generale, per gli insediamenti umani, posta da un documento dedicato allo sviluppo sostenibile, è particolarmente significativa in un momento storico in cui la previsione, al momento del Summit, era quella che, a fronte del forte incremento allora in atto del numero di persone che vivono nelle città, si riteneva che avrebbe raggiunto i 5 miliardi entro il 2030, determinando così pressioni insostenibili sui sistemi urbani. Per rispondere a tali flussi, infatti, le città sono in via di rapidissima trasformazione a ritmi sconosciuti in passato, e divengono vieppiù «micro-cosmi» dove si concentrano le più grandi sfide della vita delle persone e dove, conseguentemente, diviene sempre più importante e urgente saper rispondere a nuove esigenze. La risposta ai bisogni della città acquista una dimensione necessariamente «ultra-locale», assumen- 
do una valenza per così dire universale e, questo, non solo perché, come si avrà modo di evidenziare nel prosieguo, la gestione della città impatta su aree di più ampio respiro, ma anche perché il benessere delle persone si misura prima di tutto proprio all'interno dell'insediamento umano in cui vivono, poiché è di tale realtà che l'uomo ha, soprattutto, immediata percezione e, dunque, in riferimento al quale massimamente apprezza e valuta la qualità della vita e la dignità stessa dell'esistenza.

In effetti, la rapida crescita del numero di persone concentrate nelle città, pone una quantità di problemi che, se non adeguatamente affrontati, finiscono per compromettere la qualità della vita e la dignità delle persone, obiettivi fondamentali di tutti i documenti consacrati allo sviluppo sostenibile (a titolo meramente esemplificativo, si pensi ai problemi legati all'inquinamento, all'accesso ai servizi di base, all'insufficienza di abitazioni adeguate e alla salute pubblica).

Per questa ragione, già al punto 34, si legge espressamente che «riconosciamo che lo sviluppo e la gestione urbani sostenibili sono cruciali per la qualità della vita dei nostri concittadini», e pertanto significativamente si legge l'impegno a lavorare «con le autorità e le comunità locali per rinnovare e pianificare i nostri insediamenti umani e urbani, in modo da promuovere la coesione tra le comunità, la sicurezza personale e per stimolare l'innovazione e l'occupazione». A ben vedere, nella visione dell'Agenda, la città non è vista come entità a sé stante, ma l'attenzione verso le problematiche che le sono proprie proietta un miglioramento che impatta su realtà territoriali ben più ampie. La città è infatti, al contempo, «bersaglio» di pressioni che ne compromettono la vivibilità dignitosa ${ }^{2}$ e causa, a sua volta di squilibri di più ampia portata, ove tali pressioni siano mal gestite ${ }^{3}$.

Di sicuro interesse per le tematiche affrontate in questi incontri, è evidenziare come la prospettiva in cui l'Agenda

2. «Ridurremo gli impatti negativi delle attività urbane e delle sostanze chimiche che sono nocive per la salute umana e l'ambiente, includendo una corretta gestione a livello ambientale, l'utilizzo sicuro di sostanze chimiche, la riduzione e il riciclo dei rifiuti e l'uso più efficiente di acqua ed energia» (34).

3. «Lavoreremo per minimizzare l'impatto delle città sul sistema climatico globale» e ancora «Prederemo in considerazione gli andamenti e le proiezioni della popolazione per le nostre strategie e politiche di sviluppo urbano e rurale a livello nazionale». 
si muove mostri significative analogie con l'Enciclica Laudato si' del Santo Padre Francesco sulla cura della casa comune, sostanzialmente coeva al Summit di New York ${ }^{4}$. Prima di tutto, anche il Santo Padre si rivolge a «tutti» ${ }^{5}$, ossia non solo ai cattolici, ma ad «ogni persona che abita questo pianeta», poiché tutti siamo chiamati, in quanto esseri umani direttamente coinvolti, ad affrontare la sfida ambientale ${ }^{6}$. Deve inoltre rilevarsi come, anche in tale sede, appaiano centrali il richiamo ai temi della cooperazione e alla solidarietà, che devono necessariamente essere globali a pena della vanificazione degli sforzi, anche eventualmente cospicui, compiuti dai più per la protezione della Terra, significativamente definita quale «casa comune» (es. P.to 13 e, soprattutto, 14).

Circoscrivendo l'attenzione a quanto di rilievo in questo studio, vale la pena osservare come nella parte dedicata al «deterioramento della qualità della vita umana e degradazione sociale», è dedicata grande attenzione alle città, la cui vivibilità risulta minata dall'inquinamento originato dalle emissioni tossiche, dal caos urbano, dai problemi di trasporto e dall'inquinamento visivo e acustico, dalla mancanza di spazi verdi sufficienti (n. 44), ma anche dall'esclusione sociale, dalla disuguaglianza nella disponibilità e nel consumo dell'energia e di altri servizi, dalla frammentazione sociale, dall'aumento della violenza e dal sorgere di nuove forme di aggressività sociale, il narcotraffico e il consumo crescente di droghe fra i più giovani, la perdita di identità (n. 46). Tutti temi che, a ben vedere, sono identicamente oggetto di attenzione nell'individuazione dei 10 targets riferiti alle città sostenibili dall'Agenda 2030.

Per meglio mettere a fuoco il tema di cui si va trattando, sulla base dei 10 targets individuati all'interno dell' $11^{\circ}$ obiettivo dell'Agenda 2030, e in cui questo si scompone, è possibile rilevare come l'oggetto sia la città nel suo comples-

4. Lettera Enciclica Laudato Si' del Santo Padre Francesco sulla cura della Casa Comune, 24 maggio 2015

5. «Mi propongo di entrare in dialogo con tutti» (n. 3).

6. Sul punto, per tutti, L. De Gregorio, Laudato si': per un'ecologia autenticamente cristia$n a$, «Stato, Chiese e pluralismo confessionale», www.statoechiese.it (2016), n. 41: «la cura della creazione ognuno può partecipare con la propria cultura ed esperienza, le proprie iniziative e capacità e nessuno deve ritenersi escluso. A maggior ragione nessun credente le cui convinzioni di fede devono offrire motivazioni alte per prendersi cura della natura e collaborare come strumento di Dio». 
so, che deve ottenere attenzione nella direzione di un deciso miglioramento della qualità della vita che ivi si svolge, in primis quella degli abitanti, ma non esclusivamente, come dimostra il riferimento anche alla sicurezza e all'accessibilità ai trasporti, interessi, questi, che investono inevitabilmente anche $\mathrm{i}$ «fruitori» occasionali della città stessa.

La qualità dell'abitato diviene strumento di garanzia della qualità della vita e dell'inclusione sociale, ancora una volta mostrando una significativa analogia con l'Enciclica di Papa Francesco citata, in cui un'intera Parte (la IV) è dedicata al «Deterioramento della qualità della vita umana e degradazione sociale»; all'interno di essa, particolare enfasi è attribuita, infatti, proprio al tema della città vista come ambiente in cui massimamente l'uomo vive in una dimensione innaturale ( Non si addice ad abitanti di questo pianeta vivere sempre più sommersi da cemento, asfalto, vetro e metalli, privati del contatto fisico con la natura», n. 44) e in cui, soprattutto, si consumano politiche discriminanti, fino al punto da indicare parte dei cittadini come «i più deboli», ma anche come «gli esclusi» e financo «gli scartati della società», termini volutamente piuttosto forti che mirano ad enfatizzare le forti differenze tra uomini e le iniquità che ne derivano.

L'Agenda si mostra realmente sensibile a tali tematiche, rappresentando evidentemente la prospettiva laica del tema, ma comunque, per molteplici versi, fortemente assonante a quella religiosa.

In effetti, è significativo evidenziare come le caratteristiche strutturali della città e dei suoi servizi siano attenzionate attraverso i bisogni degli abitanti. Per rendersi conto di quanto affermato è sufficiente leggere quanto segue: "garantire a tutti l'accesso ad alloggi adeguati, sicuri e convenienti e ai servizi di base e riqualificare i quartieri poveri» (11.1); l'attenzione verso il sistema dei trasporti «con particolare attenzione ai bisogni di coloro che sono più vulnerabili, donne, bambini, persone con invalidità e anziani» (11.2) ; l'idea dell'urbanizzazione «inclusiva e sostenibile e la capacità di pianificare e gestire in tutti i paesi un insediamento umano che sia partecipativo, integrato e sostenibile» (11.3) ; attenzione per «protezione dei poveri e delle persone più vulnerabili» è anche raccomandata in riferimento "ridurre in modo significativo il numero 
di decessi e il numero di persone colpite e diminuire in modo sostanziale le perdite economiche dirette rispetto al prodotto interno lordo globale causate da calamità» (11.5) e, infine, è altresì raccomandata un'attenzione particolare ai bisogni di «donne, bambini, anziani e disabili» relativamente all'«accesso universale a spazi verdi e pubblici sicuri, inclusivi e accessibili» (11.7).

\section{La protezione del patrimonio culturale come strumento di} realizzazione di «città sostenibili»

A parere di chi scrive, merita particolare attenzione il fatto che tra i traguardi previsti nell'ambito dell' $11^{\circ}$ obiettivo, il Programma menzioni anche la necessità di «rafforzare gli sforzi di protezione e conservazione del patrimonio culturale e naturale mondiale»

Linteresse per questa affermazione deriva dal fatto che, ancorché nel programma del 2015 essa non costituisca l'unico riferimento alla cultura intesa nel suo significato più generale ${ }^{7}$, tuttavia si tratta del solo riferimento alla protezione del patrimonio culturale in quanto strumentale al perseguimento di obiettivi di sviluppo sostenibile ${ }^{8}$.

Prima di qualunque ulteriore approfondimento sul tema, conviene precisare come, secondo chi scrive, il concetto di «patrimonio culturale» contenuto nel Programma del 2015 sembri coincidere con quello di «patrimonio urbano» di cui alla raccomandazione Unesco del 2011 concernente il paesaggio urbano storico, secondo la quale «il patrimonio urbano costituisce per l'umanità un bene sociale, culturale ed economico, definito da una stratificazione storica di valo-

7. Si adotta in questa sede l'idea di cultura nella sua accezione più ampia. Tra le prime definizioni, si deve menzionare quella contenuta nella Dichiarazione di Città del Messico sulle Politiche Culturali, adottata dalla Conferenza mondiale, il 6 agosto 1982, come «l'ensemble des traits distinctifs, spirituels et matériels, intellectuels et affectifs, qui caractérisent une société ou un groupe social englobant aussi outre les arts et les lettres, les modes de vie, les droits fondamentaux de l'être humain, les systèmes de valeurs, les traditions et les croyances».

8. Nello stesso tempo - come sottolineato nell'edizione speciale del 2013 del Rapporto delle Nazioni Unite sull'economia creativa: ampliare i percorsi di sviluppo locale - le città giocano un ruolo sempre più importante come attori del cambiamento nella direzione dello sviluppo sostenibile anche grazie alla cultura che porta un valore aggiunto, in termini monetari e non monetari, attraverso le espressioni culturali, la salvaguardia del patrimonio materiale e immateriale, la promozione della diversità culturale, l'urbanistica $\mathrm{e}$ l'architettura. 
ri che sono stati prodotti dal succedersi di culture passate e contemporanee e un accumulo di tradizioni e di esperienze, riconosciute come tali nella loro diversità»; dunque, esso fa riferimento non solo al patrimonio mondiale dell'umanità (come definito nella convenzione UNESCO per la protezione del patrimonio mondiale, culturale e naturale del 1972), ma altresì ad un concetto più ampio e maturo, che include altresì (allo stesso modo che il patrimonio storico artistico), il patrimonio immateriale (conformemente alla convenzione UNESCO per la salvaguardia del patrimonio culturale immateriale del 2003) e le differenze culturali che, a partire dalla Convenzione UNESCO del 2005 sulla protezione e la promozione delle diversità delle espressioni culturali del 2005, divengono esse stesse una ricchezza da salvaguardare.

Ancora una volta può senz'altro rimarcarsi l'assonanza con le parole del Santo Padre. Nel Cap. IV dell'Enciclica, significativamente intitolato «Un'ecologia integrale», si afferma espressamente come «Insieme al patrimonio naturale, vi è un patrimonio storico, artistico e culturale, ugualmente minacciato. È parte dell'identità comune di un luogo e base per costruire una città abitabile» (corsivo di chi scrive) e tale patrimonio va inteso non solo «come i monumenti del passato, ma specialmente nel suo senso vivo, dinamico e partecipativo, che non si può escludere nel momento in cui si ripensa la relazione dell'essere umano con l'ambiente» (n. 143). L'affermazione è pregnante. La conservazione dell'identità del luogo diviene elemento fondamentale per assicurare la vivibilità della città («per costruire una città abitabile», secondo le precise parole di Papa Francesco), posto, tra l'altro che «La scomparsa di una cultura può essere grave come o più della scomparsa di una specie animale o vegetale» (n. 145). Il consumismo e la globalizzazione, in particolare, minacciano la ricchezza e la varietà delle culture, rischiando di appiattirle e di portare allo smarrimento irreversibile di queste nel segno di una omologazione globale (n. 144), secondo un'affermazione fortemente assonante, seppur proiettata su un piano molto più ampio e generale, con quanto rilevato nel Preambolo della Convenzione Unesco sulla protezione e la promozione della diversità delle espressioni culturali del 20 ottobre $2005^{9}$.

9. «Constatando che i processi di globalizzazione, agevolati dalla rapida evoluzione delle tecnologie dell'informazione e della comunicazione, se hanno contribuito a stabilire 
Ciò detto, l'attenzione dell'Agenda 2030 per il patrimonio culturale delle città e degli insediamenti umani è degna di nota almeno per due ragioni.

La prima. È la prima volta che un Summit mondiale specificamente dedicato allo sviluppo sostenibile attribuisce esplicitamente alla protezione del patrimonio culturale un ruolo specifico in vista della realizzazione degli obiettivi che si propone. In effetti si deve sottolineare come i Summit precedenti non avessero mai affrontato direttamente la questione, concentrandosi piuttosto sull'ambiente in senso ecologico più che culturale in senso proprio.

Riassumendo molto brevemente le tappe del percorso evolutivo, deve necessariamente sottolinearsi come questa attenzione fosse assente nel citato Rapport Bruntland del 1987 (Report Our Common Future) sia nella Dichiarazione di Rio di Janeiro sull'ambiente e lo sviluppo del 1992; in particolare, essa mancava altresì nella Dichiarazione di Johannesburg del 2002, ancorché avesse esplicitamente ancorato lo sviluppo sostenibile alla dignità umana, il cui perseguimento, secondo l'atto istitutivo dell'UNESCO, presuppone precisamente la diffusione della cultura ${ }^{10}$.

condizioni inedite capaci di consolidare l'interazione interculturale, rappresentano anche una sfida per la diversità culturale, segnatamente nell'ambito dei rischi di squilibrio fra Paesi ricchi e Paesi poveri»

10. Si legge in effetti nel Trattato Istitutivo dell'Unesco, firmato il 16 novembre 1945, che il percorso di costruzione di pace dovesse necessariamente passare per la «solidarietà intellettuale e morale dell'umanità», non essendo in alcun modo sufficiente «una pace fondata soltanto sugli accordi economici e politici dei governi» che «non potrebbe ottenere l'adesione unanime, duratura e sincera dei popoli»; è per questa ragione che «l'Organizzazione si propone di contribuire al mantenimento della pace e della sicurezza favorendo, attraverso l'educazione, la scienza e la cultura, la collaborazione tra le nazioni, onde garantire il rispetto universale della giustizia, della legge, dei diritti dell'uomo e delle libertà fondamentali che la Carta delle Nazioni Unite riconosce a tutti i popoli, senza distinzione di razza, sesso, lingua o religione» e a tale scopo l'Organizzazione: «a) Favorisce la conoscenza e la comprensione reciproca delle nazioni, prestando la sua opera agli organi di informazione di massa; a questo scopo essa sollecita quegli accordi internazionali che ritiene utili per facilitare la libera circolazione del pensiero, attraverso la parola o l'immagine; b) imprime un'efficace impulso all'educazione popolare ed alla diffusione della cultura», ma soprattutto, per quanto di maggiore interesse in questa sede $\ll c)$ Aiuta il mantenimento, il miglioramento e la diffusione del sapere: Vegliando sulla conservazione e protezione del patrimonio universale di libri, opere d'arte, monumenti di interesse storico o s scientifico, raccomandando ai popoli interessati delle convenzioni internazionali a tale effetto; favorendo la cooperazione tra le nazioni in tutti i settori dell'attività intellettuale e lo scambio internazionale sia di rappresentanti dell'educazione, della scienza e della cultura che di pubblicazioni, opere d'arte, materiale di laboratorio e di ogni altra utile documentazione; facilitando con adeguati metodi di 
Prima del Programma del 2015, solamente nella Dichiarazione di Rio de Janeiro del 2012, poteva leggersi una dichiarazione specifica sulla cultura («Sottolineiamo l'importanza della cultura per lo sviluppo sostenibile»), ma questa affermazione non era poi sviluppata nel testo.

Tale considerazione porta alla seconda delle ragioni per la quale conviene sottolineare l'esistenza di un riferimento espresso alla protezione del patrimonio culturale nel corpo dell'Agenda 2030.

Sembra possibile credere che questa dichiarazione sia piuttosto dovuta all'influenza delle Convenzioni UNESCO in seno alle quali il tema dello sviluppo sostenibile e quello della protezione del patrimonio culturale hanno seguito vie convergenti.

In particolare, la Convenzione sulla protezione e la promozione della diversità delle espressioni culturali del 2005, è la prima a prevedere un principio autonomo che integra espressamente i due temi: «Principio di sviluppo sostenibile. La diversità culturale rappresenta un patrimonio sostanziale per gli individui e le società. Lo sviluppo sostenibile, di cui beneficiano le generazioni presenti e future, presuppone la protezione, la promozione e il mantenimento della diversità culturale» [principio n. 6 $]^{11}$.

Ma per ciò che concerne queste riflessioni, è la Dichiarazione di Hangzhou il passaggio più interessante, perché in essa, finalmente, la cultura è indicata espressamente come chiave dello sviluppo sostenibile. Adottata in sede Unesco ad Hangzhou (nella Repubblica popolare della Cina) il 17 maggio 2013, in occasione del Congresso Internazionale su «La cultura: chiave dello sviluppo sostenibile», la Dichiarazione costituisce un vero e proprio appello ai vari paesi del mondo affinché pongano la «cultura» nella posizione principale della politica sullo sviluppo sostenibile, come allora era stato configurato in occasione dei Summit mondiali sul tema, e, in particolare, affinché la cultura sia inclusa come quarto principio fondamentale nell'Agenda delle nazioni Unite per

cooperazione internazionale l'accesso di tutti i popoli a quanto pubblicato da ciascuno di essi».

11. Su questi temi, sia consentito rinviare a C. Videtta, Cultura e sviluppo sostenibile. Alla ricerca del IV pilastro, Giappichelli, Torino 2018. 
lo sviluppo post 2015, al pari dei diritti dell'uomo, dell'uguaglianza e della sostenibilità.

La Dichiarazione del 2013 rivendica, infatti, un preciso ruolo per la cultura nella lotta contro quelle piaghe dell'umanità già più volte evidenziate nelle Dichiarazioni da Rio 1992 a quella, che sarà adottata pochi anni dopo, di New York 2015, ossia la crescita della popolazione, l'urbanizzazione, il degrado ambientale, le catastrofi, i cambiamenti climatici, l'aggravarsi delle disuguaglianze e il persistere della povertà.

In tale contesto, proprio le città divengono oggetto di attenta considerazione all'interno delle azioni finalizzate a mettere la cultura al centro delle politiche dello sviluppo sostenibile.

In particolare, la $8^{\circ}$ azione, afferma la necessità di «servirsi della cultura come risorsa per realizzare uno sviluppo e una gestione sostenibile delle aree urbane». In tale sezione, l'attenzione è rivolta in particolare alla qualità degli «ambienti storici», che, conservati in armonia con il loro contesto naturale, rappresentano la chiave delle città sostenibili. La cultura si afferma dunque come uno strumento non solo di sviluppo economico, ma soprattutto di promozione di dialogo, di rispetto della diversità, di inclusione sociale e dunque di coesione.

A ben vedere, sembra possibile affermare come l'azione a livello locale accolga quell'idea di cultura vista come strumento per attuare percorsi di pace tra i popoli, concetto, questo, alla base dell'atto istitutivo dell'Unesco del 1945 e soprattutto della Convenzione Unesco del 1972 sulla protezione del patrimonio mondiale culturale e naturale.

Dunque, la cultura (e in particolare la protezione del patrimonio culturale) diviene uno strumento privilegiato per favorire il dialogo a livello mondiale ed egualmente a livello locale, in particolare all'interno delle città, dove, tra l'altro, il processo di immigrazione massiccia può contribuire in misura non indifferente a generare tensioni identitarie che finiscono per minare la stessa qualità della vita.

Tali temi sono ulteriormente sviluppati in occasione della Conférence Internationale de Hangzhou sur «La cultura per le città sostenibili» (10-12 dicembre 2015), all'indomani dell'adozione dell'Agenda 2030 per lo sviluppo sostenibile. Nelle Conclusioni, si può leggere una conferma della posizione 
già espressa nel 2013, particolarmente dove si raccomanda di tener conto del fatto che «i modelli urbani sono differenti e che il cambiamento dinamico delle città è costante»e che pertanto si sostiene la necessità di evitare «modelli standardizzati e prescrittivi in favore di un approccio integrato, innovativo e adattativo ai differenti contesti».

In tale ambito, la raccomandazione di integrare il patrimonio culturale nella «nuova agenda urbana» significa, allo stesso tempo, raccomandare la protezione del patrimonio culturale esistente nelle città quale espressione di identità di queste e significa altresì permettere alle persone di modellare i propri ambienti urbani.

È senza dubbio interessante sottolineare come i due aspetti citati siano strettamente legati, atteso che questo chiarisce il senso della protezione del patrimonio culturale all'interno delle città: in effetti, se, come è innegabile, le città sono in costante trasformazione (soprattutto in questo periodo storico), il patrimonio culturale rappresenta la continuità verso il passato, il patrimonio identitario da trasmettere alle generazioni future, senza tuttavia negare la trasformazione, poiché il patrimonio culturale (nel suo più ampio significato) è, in quanto tale, in continua evoluzione.

Nel 2016, la Conferenza delle Nazioni Unite sugli insediamenti urbani e lo sviluppo urbano sostenibile (Habitat III), tenutasi a Quito dal 17 al 20 ottobre, adotta un Nuovo Programma per le città che riafferma l'impego mondiale a promuovere uno sviluppo urbano sostenibile come prescritto dall' $11^{\circ}$ obiettivo dell'Agenda 2030.

In particolare, anche il Nuovo Programma contiene dei riferimenti significativi al patrimonio culturale.

In effetti, tra gli «impegni portatori di cambiamento a favore dello sviluppo urbano sostenibile», si riafferma il ruolo fondamentale del patrimonio culturale in vista di politiche di «recupero e rivitalizzazione delle aree urbane» e di rafforzamento della «partecipazione sociale e l'esercizio della cittadinanza d'inclusione sociale» (par. 38).

In tal senso, sono formulate raccomandazioni specifiche («per una messa in opera efficace») relativamente alla «pianificazione e gestione dello sviluppo dello spazio urbano», all'interno del quale si ritrova l'impegno preciso a fare «della cultura una componente prioritaria nei piani e nelle strategie urbane quando si adottano strumenti di pianificazio- 
ne» che devono preservare «un largo ventaglio di paesaggi e di beni del patrimonio culturale materiale e immateriale e proteggerlo contro eventuali effetti perturbatori dello sviluppo urbano» (par. 124).

Quindi, se - come aveva già sottolineato la Convenzione di Hangzou del 2013 - le pressioni sulle città derivano da una massiccia urbanizzazione associata a pressioni economiche, sarà importante che i piani di gestione dello sviluppo urbano affrontino il problema.

Tali raccomandazioni meritano di essere menzionate poiché, ancorché esse costituiscano essenzialmente le sole indicazioni concernenti la protezione del patrimonio culturale nell'intero documento, tuttavia forniscono alcune linee concrete d'azione, praticamente assenti in tutti gli altri documenti.

Su queste questioni, è sicuramente interessante evidenziare come tale raccomandazione sia espressione diretta di un principio generale di integrazione dei temi della cultura (e della protezione del patrimonio culturale) nello sviluppo sostenibile, già, tra l'altro, prescritto sia dalla Convenzione europea sul Paesaggio $(2000)^{12}$ sia dalla Convenzione-quadro sul valore del patrimonio culturale per la società (adottata a Faro nel 2005) ${ }^{13}$, entrambe del Consiglio d'Europa.

Invero, ancorché le due convenzioni non siano specificamente consacrate alle «città sostenibili», in ogni caso forniscono delle indicazioni particolarmente interessanti per meglio comprendere la relazione tra il patrimonio culturale e lo sviluppo sostenibile.

Come già in altra sede si è rilevato ${ }^{14}$, infatti, i due preamboli consacrano un legame tra il patrimonio culturale/ paesaggio e lo sviluppo sostenibile, indicando soprattutto come la relazione cultura/paesaggio rappresenti una risorsa

12. Sul punto, per tutti, F. Cartei (a cura di), Convenzione europea del paesaggio e governo del territorio, Bologna 2007; S. Foà, Dalla Convenzione europea al Codice dei beni culturali e del paesaggio. Obiettivi di tutela e valorizzazione, in A. Crosetti (a cura di), La tutela della natura e del paesaggio, Trattato di diritto dell'ambiente (a cura di R. Ferrara, M. A. Sandulli), vol. III, Milano 2014, pp. $431 \mathrm{sgg}$.

13. C. Carmosino, La Convenzione quadro del Consiglio d'Europa sul valore del patrimonio culturale per la società, "Aedon", www.aedon.mulino.it, n. 1/2013; P. Carpentieri, La Convenzione di Faro sul valore dell'eredità culturale per la società (da un punto di vista logico), «federalismi. it», www.federalismi.it (2017), n. 4.

14. C. Videtta, Cultura e sviluppo sostenibile cit., pp. $76 \mathrm{sgg}$. 
di sviluppo sostenibile e di qualità della vita in una società in costante evoluzione.

In particole, nella Convenzione europea sul paesaggio (2000), si può leggere che «Ogni Parte si impegna a [...] integrare il paesaggio nelle politiche di uso del suolo, di urbanistica e nelle politiche culturali, ambientali, agricole, sociale et economiche, così come nelle altre politiche che possono avere un affetto diretto o indiretto sul paesaggio»; allo stesso tempo, a livello più generale, la Convenzione quadro di Faro, ancorché essa non parli nello specifico di città sostenibili, stabilisce comunque una sorta di principio generale di integrazione, esplicitamente collegato a quello più specifico concernente i piani urbani di cui si è detto: "per mantenere il patrimonio culturale, le parti si impegnano [...] ad assicurare che i bisogni specifici della conservazioni del patrimonio culturale siano tenuti in considerazione in tutte le discipline tecniche generali» (art. 9, lett. c) e altresì che «al fine di valorizzare il potenziale del patrimonio culturale in quanto fattore di sviluppo economico sostenibile, le parti si impegnano a fare in modo che tali politiche rispettino l'integrità del patrimonio culturale senza compromettere i suoi valori intrinseci» (art. 10, lett. c).

3. Qualche riflessione sullo stato dell'arte in Italia. Solo retorica? In Italia, la «protezione e conservazione del patrimonio culturale» di cui all'Agenda 2030 è, in larga misura, affidata al Codice dei beni culturali e del paesaggio, d.lgs. 22 gennaio 2004 n. 42. Il modello adottato dal testo citato, tuttavia, è improntato a fornire una tutela per così dire «puntiforme», appuntata su singole e circoscritte realtà; resta viceversa estranea ad esso la possibilità di trovare applicazione a realtà complesse come $\mathrm{i}$ tessuti cittadini ${ }^{15}$. È ben vero che l'obiettivo 11.4 dell'Agenda 2030 (e, d'altro canto, anche le stesse Convenzioni Unesco citate) non richiedono necessariamente una disciplina di protezione del patrimonio culturale che riguardi la città nel suo complesso, ma lasciano ampio spazio alle scelte discrezionali dei singoli Stati circa la scelta delle soluzioni normative più adeguate onde

15. Per rilievi critici sul punto, sia consentito rinviare, anche per l'apparato bibliografico, a C. Videtta, I centri storici al crocevia tra disciplina dei beni culturali, disciplina del paesaggio $e$ urbanistica: profili critici, «Aedon», www.aedon.mulino.it (2012), n. 3. 
perseguire gli obiettivi indicati; tuttavia, la mancanza di una programmazione adeguata che guardi comunque agli interventi «nella città» e non alle singole emergenze, vista unitamente alla crisi fiscale generale, impattano, a parere di chi scrive, sulla effettiva conservazione di un patrimonio culturale cittadino, nel senso più pieno della parola.

Purtroppo le forti pressioni economiche e i processi di globalizzazione in atto inducono, più massicciamente che in passato, a considerare il patrimonio culturale soprattutto come una risorsa nel senso economico del termine, il che genera troppo spesso effetti devastanti sulla sua protezione.

Ben si può dire, in effetti, che, malgrado l'art. 2 del Codice dei beni culturali e del paesaggio affermi espressamente che «la valorizzazione è effettuata in forme compatibili con la tutela e in modo da non comprometterne i bisogni», non è tuttavia raro trovare decisioni amministrative o atti legislativi nei quali le ragioni della tutela sono sacrificate alle ragioni, neppure sempre evidenti, dello sviluppo economico ${ }^{16}$.

Sotto altro, ma convergente profilo, vale la pena rilevare come l'Agenda urbana per l'UE, patto di Amsterdam, adottato nel corso della riunione informale dei Ministri Europei responsabili degli affari urbani (30 maggio 2016), rimarchi il fatto che la coesione economica, sociale e territoriale dell'UE e la qualità della vita dei suoi abitanti dipendono largamente dal successo dello sviluppo urbano sostenibile (nel cui contesto, giova ribadirlo, anche la tutela del patrimonio culturale gioca un ruolo assai significativo); vi si ricorda altresì, l'importanza strategica di un approccio equilibrato, durevole e integrato delle problematiche urbane che dovrebbero coprire tutti i principali aspetti dello sviluppo urbano (in particolare economico, ambientale, sociale, territoriale ed anche culturale) al fine di garantire una solida governance e politica urbana.

Lapproccio comune tra le politiche settoriali e i differenti livelli amministrativi diviene la chiave delle città sostenibili: tutti i livelli di governo devono essere coinvolti e deve essere garantito il coordinamento e l'interazione efficace 
all'interno dei settori, nel pieno rispetto del principio di sussidiarietà e delle competenze di ciascun livello.

Per far fronte alle sempre più complesse sfide delle aree urbane e promuoverne il progresso ambientale, economico, sociale e culturale, le autorità urbane devono cooperare con le comunità locali, società civile, società e centri di competenza, che sono tutti i principali attori dello sviluppo sostenibile.

Deve purtroppo rilevarsi come la legislazione italiana sia piuttosto indietro sul punto e, per quanto qui di interesse, non sia del tutto in grado di prendersi cura del patrimonio culturale delle città (come realtà specifiche) al fine di raggiungere città veramente sostenibili, anche a causa di evidenti problemi di competenze amministrative sovrapposte (paesaggio, patrimonio storico-artistico/ambiente/governo del territorio...).

Significativamente, in Habitat III - Rapport national de l'Italie de 2016, si può leggere che «le regioni hanno sino ad ora elaborato regolamentazioni che hanno modificato la tipologia degli strumenti di pianificazione urbana e certe ipotesi (per es. la classificazione di certe parti di territorio), ma non sono state in grado di influenzare la disciplina della proprietà e molto poco sulla disciplina di aspetti di interesse generale come l'ambiente, il paesaggio e il patrimonio culturale, che rientrano nella giurisdizione nazionale».

Anche la Carta di Bologna per l'ambiente del 2017, firmata dai Comuni e dalle città metropolitane che impegna le grandi città italiane a perseguire gli obiettivi di protezione ambientale in conformità agli obiettivi di sviluppo sostenibile, mette l'accento su questioni come uso sostenibile del territorio, economia circolare, adattamento ai cambiamenti climatici, politica energetica, qualità dell'aria e dell'acqua, ecosistemi, protezione ambientale, biodiversità e mobilità sostenibile, ma non menziona la protezione del patrimonio culturale.

Infine, significativamente nel rapporto ISTAT 2019, Informazioni statistiche sul programma 2030 in Italia, si può leggere che le spese pubbliche per abitante consacrate alla protezione della biodiversità e dei paesaggi sono diminuiti rispetto a dieci anni fa. Da notare come il rapporto contenga effettivamente anche dei riferimenti all'importanza della protezione del patrimonio culturale, ma essi sono qua- 
si sempre legati al perseguimento di obiettivi di turismo sostenibile e dunque di sviluppo economico.

Qualche riferimento più generale a questo tema è fatto, a proposito dell'obiettivo 11 dell'Agenda 2030; a ben vedere, tuttavia, pare semplicemente la ripetizione dell'impegno già preso a livello internazionale, con il solo accento supplementare sull'importanza degli interventi volti a rinforzare la capacità di pianificazione necessaria a far fronte alla portata e alla complessità delle questioni di sostenibilità urbana e della gestione partecipativa e integrata.

Sulla base di quanto osservato, pare dunque doversi rilevare mestamente come il ruolo della protezione del patrimonio culturale come strumento per perseguire modelli di città sostenibili, sembri in verità, almeno per il momento e salve alcune eccezioni, limitato a una dimensione, più che altro, teorica (per non dire retorica).

In effetti troppo spesso, il patrimonio storico-artistico non è tutelato che al fine di garantire flussi turistici, il che può però avere impatti negativi sulla conservazione del patrimonio stesso, ma soprattutto porta ad un disinteresse per i beni situati nelle zone meno attrattive turisticamente della città (in particolare nelle zone periferiche); queste ultime sono spesso completamente abbandonate poiché meno visibili, e dunque non utili allo sviluppo economico-turistico delle città stesse, il che contrasta totalmente con il ruolo che ad esse è attribuito, come si è detto, negli atti internazionali dedicati alle città sostenibili. Risulta così ancora largamente offuscato il ruolo del patrimonio culturale in quanto strumento (indispensabile) di promozione di cultura, chiaramente affermato dall'art. 9 Cost. («La Repubblica promuove lo sviluppo della cultura e la ricerca scientifica e tecnica. Tutela il paesaggio e il patrimonio storico e artistico della Nazione») specie nel momento in cui esso assume la pericolosa veste di «petrolio d'Italia», smarrendosi troppo spesso il ruolo che esso può svolgere a livello di strumento di promozione di processi inclusivi e di pace di cui si è detto. 
Il tema proposto ha come obiettivo quello di mettere in relazione due aspetti, la crescita economica e lo sviluppo solidale. La ragione, o una delle ragioni che si pongono alla base di questa riflessione, riposa nel fatto che il modello che presiede i sistemi economici attuali - quanto meno nei paesi occidentali - è di un' economia di mercato di tipo capitalistico basato principalmente sulla necessità di incrementare nel tempo la crescita quantitativa della produzione e lascando al sistema nel suo insieme la regolazione nella distribuzione delle risorse prodotte (il c.d. trickle down effect) ${ }^{1}$.

Gli storici dell'economia hanno sempre messo in guardia dall'uso disinvolto della parola "capitalismo", concetto assai difficile da definire e multiforme ${ }^{2}$. Ci sono tante e svariate forme di capitalismo. Il capitalismo dell'America degli

1. La teoria del trickle-down, in voga soprattutto negli Stati Uniti, associa direttamente la crescita economica ad un contemporaneo miglioramento della condizione economica dell'intera popolazione, senza valutare eventuali squilibri nella distribuzione dei redditi e della ricchezza. Tale teoria è normalmente associata alla reaganomics e al liberismo più integrale.

2. Per un approfondimento sul tema si rimanda a F. Braudel, La dinamica del capitalismo, il Mulino, Bologna, 1988; 
anni Quaranta-Sessanta ha, ad esempio, poco in comune con il supercapitalismo degli ultimi trent'anni. Inoltre il capitalismo non è solo un modello che definisce gli aspetti economici di una società. Troppo spesso, infatti, si usa il concetto senza conoscerne approfonditamente il contenuto, se non per qualche elemento basilare come l'importanza della proprietà privata e della libera iniziativa, la centralità dei consumi e la rilevanza del modello di mercato. Il capitalismo non ha una sola forma (quella che caratterizza la società odierna), ma se ne possono avere diverse, ognuna con elementi propri; inoltre non rimane sempre uguale a sé stesso come definito dalle sue prime affermazioni, ma muta e si evolve - o forse involve -; esso non riguarda solo il denaro e il modo in cui vengono prodotti i beni, ma ha a che fare anche con le idee e la cultura. Non è, dunque, un monolite immobile e immutabile, ma nel corso della storia si è assistito al susseguirsi di capitalismi, con strutture interne differenti e capaci di avere conseguenze, anche molto diverse tra loro, sulla società e sull'economia. Non si limita inoltre ad influenzare la sola sfera economica, ma si accompagna anche ad un'ideologia che interviene sulle categorie culturali di una società, plasmandole e modificandole ${ }^{3}$.

Il concetto di capitalismo non va confuso con quello di economia di mercato. Si tratta di cose molto diverse; l'economia di mercato è una forma antichissima di organizzare la funzione produttiva e di scambio attraverso la libera iniziativa e azione dei singoli operatori, che comunicano tra loro tramite la quantità e il prezzo di beni e servizi. L'economia di mercato, dunque, ha permesso la civilizzazione delle comunità riconoscendo la dignità delle persone le quali, liberate dal giogo delle logiche feudali, hanno messo in campo le loro capacità creative nel dare forma e sostanza a nuove relazioni non solo economiche ma anche umane e inclusive. È attraverso l'industrializzazione e il ruolo crescete del capitale che nasce gradualmente, a partire dalla metà del Settecento, il capitalismo in senso proprio. Mentre nel modello di economia di mercato l'obiettivo era quello di servire la persona e aiutarla a vivere una vita degna, nel

3. Interessante, in proposito, il saggio di W. Benjamin, "Kapitalismus als Religion" (Capitalismo come religione) del 1921, pubblicato in Gesammelte Schriften, a cura di R. Tiedemann e H. Schweppenhäuser, Suhtkamp, Frankfurt a.M. 1985, vol. VI, pp. 100-103. 
modello capitalistico la produzione diventa fine a sé stessa. In proposito Sturzo, negli anni Venti, scriveva che non bisogna avere paura del capitalismo; come un grande fiume, esso può scorrere portando benefici. Ammoniva però, sempre nella metafora, che bisogna avere molta cura degli argini per evitare che esca impetuoso dall'alveo e travolga ogni cosa. La storia degli ultimi anni ha visto ogni argine travolto; al centro dell'economia non si è messa la persona ma neppure la produzione. Al centro è stato posto il capitale finanziario, ossia quello che Gallino argutamente definisce "finanzcapitalismo".

Come macchina sociale, il finanzcapitalismo ha superato ciascuna delle precedenti, [...], a motivo della sua estensione planetaria e della sua capillare penetrazione in tutti i sottosistemi sociali, e in tutti gli strati della società, della natura e della persona. Perché il finanzcapitalismo ha come motore non più la produzione di merci ma il sistema finanziario. Il denaro viene impiegato, investito, fatto circolare sui mercati allo scopo di produrre immediatamente una maggior quantità di denaro. In un crescendo patologico che ci appare sempre più fuori controllo. ${ }^{4}$

Tale modello di capitalismo finanziario è scaturito, dagli anni ottanta in avanti, dalla crisi di una forma precedente, chiamato capitalismo societario (o produttivo), che aveva caratterizzato il mondo occidentale nel secondo dopoguerra. In esso preponderante era il ruolo delle istituzioni dello stato nazionale, le quali avevano come obiettivo quello di creare condizioni di vita stabili e sicure, dopo i tragici eventi della prima metà del secolo. Il fine perseguito era di tenere insieme lo sviluppo economico e l'integrazione sociale. Questi furono gli anni, oltre che di crescita in ricchezza e possibilità, anche della creazione del welfare state, che andava a completare il processo di formazione di democrazie basate sui diritti individuali, con lo sviluppo di un sistema sanitario nazionale, la riforma del mercato del lavoro e i sistemi di protezione sociale. L'enorme apparato istituzionale svolgeva un ruolo essenziale per l'economia, attraverso investimenti pubblici per favorire lo sviluppo e, inoltre, per la collettività attraverso la risoluzione dei maggiori problemi sociali e svolgendo 
anche un fondamentale compito culturale, in quanto era in grado di connettere i diversi ruoli sociali con significati etici e simbolici condivisi. I successi raggiunti in tale periodo furono molteplici ed evidenti, rispetto al miglioramento della qualità della vita dei cittadini delle società occidentali. Un intervento, però, così massiccio dello Stato nei diversi campi della vita sociale, attraverso ruoli rigidi ed esigenti, a tratti soffocanti, era destinato a divenire il punto debole del sistema stesso. Gli enormi sviluppi del secondo dopoguerra, ininterrotti fino agli anni settanta, portarono anche un'estensione dello spazio di autonomia e delle possibilità del singolo, favorendo con ciò una sempre maggiore richiesta di libertà. I traguardi del capitalismo societario hanno, quindi, generato lo stesso elemento che avrebbe sancito la sua crisi. Infatti, la tendenza invasiva delle istituzioni venne vista sempre più come limite per la libertà degli individui, diventati, nel frattempo, maggiormente consapevoli di sé. I primi segnali di crisi si manifestarono a partire dagli anni Sessanta e Settanta del secolo scorso, diventando espliciti negli anni Ottanta. L'economia iniziò a subire una fase di arresto e poi di crisi - la prima dalla guerra -, gli Stati e i governi fecero sempre maggiore fatica a dare risposte alle problematiche di un mondo costantemente più complesso e connesso. Tali tensioni nell'ultimo decennio del Novecento portarono a un superamento del sistema vigente, attraverso quella che sembrava essere la risposta più adatta alle sue sfide che poneva la nuova società globalizzata: la dottrina e pratica economico-politica del neoliberismo.

$\grave{E}$, quindi, solo a partire da una trentina di anni che iniziarono a porsi le basi per lo sviluppo di una nuova struttura economica e sociale. Il capitalismo attuale è stato generato da precise scelte politiche ed economiche, operate sulla base di dottrine neoliberiste tra la fine degli anni Ottanta e l'inizio degli anni Novanta; nel giro di trent'anni ha cambiato radicalmente il mondo e soprattutto evidenziato, anche in maniera devastante (come la crisi scoppiata nel 2008), tutti i limiti e le conseguenze negative.

\section{Crescita e sviluppo}

Crescita e sviluppo non sono sinonimi. La crescita nella sua accezione economica è l'aumento di beni e servizi prodotti dal sistema economico in un dato periodo di tempo. Lo sviluppo è una vasta branca delle scienze sociali che nasce nel 
preciso contesto storico-politico del secondo dopoguerra. Facendo riferimento ad una distinzione comune, la crescita viene riferita alla quantità di beni e servizi disponibili, mentre lo sviluppo comprende anche elementi di qualità della vita di natura sociale, culturale e politica.

La crescita economica è generalmente riferita alla capacità di un sistema economico di incrementare la disponibilità di beni e servizi atti a soddisfare il fabbisogno di un data popolazione. Si suppone che la disponibilità di beni e servizi debba aumentare nel tempo, in quanto tendenzialmente cresce la popolazione e con essa la domanda di beni. La crescita economica è inoltre l'indicatore economico maggiormente utilizzato dagli economisti, dai governi e dalle organizzazioni economiche internazionali, ed è spesso associata al benessere della popolazione. Tuttavia, la relazione tra le misure della crescita generalmente in uso e il benessere è molto complessa e controversa. Analogamente, si tende a considerare la crescita economica come sinonimo di sviluppo. Ma lo sviluppo è un concetto più ampio e comprende anche altri fattori, come la salute, l'educazione, le relazioni interpersonali.

Il tema è noto in letteratura fin dal 1974 quando l'economista Richard Easterlin illustrò il paradosso tra reddito e felicità ${ }^{5}$. La tesi dimostra che, fino ad un certo punto, la crescita del reddito pro capite si accompagna con una crescita della soddisfazione percepita. Oltre quel livello, la curva inizia a decrescere in quanto entrano in gioco altri fattori che diventano più importanti dell'aumento della disponibilità monetaria.

Il mito della crescita deve quindi essere contemperato con altri elementi fondamentali per rendere lo sviluppo davvero sostenibile. Si tratta, quindi, di considerare la dimensione economica in relazione con la dimensione ambientale e la dimensione sociale; l'aspetto economico, che ha come vettore la crescita al fine di garantire alla popolazione i beni necessari per il suo sostentamento, deve armonizzarsi con il rispetto delle politiche ambientali per non depauperare l'ecosistema nel quale le persone vivono e delle politi-

5. R.A. Easterlin, Does Economic Growth Improve The Human Lot? Some Empirical Evidence, in P.A. David, M.W. Reder (eds), Nations and Households in economic growth: Essays in honor of Moses Abromowitz, Academic Press Inc., New York 1974. 

e sviluppo

solidale che sociali al fine di includere tutte le persone quali destinatarie della redistribuzione della ricchezza prodotta e nel garantire livelli accettabili di beni pubblici e di beni comuni.

Si tratta, quindi, di superare il Prodotto Interno Lordo (Pil) come unico indicatore per valutare il benessere di una nazione e considerare indicatori più complessi in grado di tenere conto degli aspetti sottolineati poc'anzi. Un indicatore sviluppato dall'Istat e dal Cnel in questo senso è il Bes (Benessere Equo e Sostenibile) che, nel considerare congiuntamente gli aspetti economici, ambientali e sociali, è corredato da misure di disuguaglianza e di sostenibilità.

Questo indicatore ${ }^{6}$ è poco conosciuto dall'opinione pubblica e dai mezzi di informazione in generale; rimane una misura consultata dagli addetti ai lavori ma poco diffusa nella conoscenza collettiva.

È necessario, quindi, iniziare un lavoro culturale importante che faccia prendere coscienza alle persone dei reali andamenti dei fattori che generano/distruggono benessere, per metterle in grado di valutare con maggior consapevolezza le politiche economiche, sociali e ambientali che nei diversi paesi/territori vengono proposte ed applicate.

Come noto, l'Assemblea Generale delle Nazioni Unite ha approvato all'unanimità il 25 settembre 2015 l'Agenda 2030 per lo sviluppo sostenibile, composta da diciassette Obiettivi di Sviluppo Sostenibile (Sustainable Development Goals, Sdgs) e di centosessantanove sub-obiettivi (target). Il documento di presentazione dell'Agenda, Transforming our world: the 2030 Agenda for Sustainable Development si apre con le seguenti parole:

Questo programma è un piano d'azione per la gente, il pianeta e la prosperità. Inoltre, cerca di rafforzare la pace universale in un contesto di maggiore libertà. Riconosciamo che lo sradicamento della povertà in tutte le sue forme e dimensioni, inclusa la povertà estrema, è la più grande sfida globale e un requisito indispensabile per lo sviluppo sostenibile. Tutti i paesi e tutte le parti interessate, agendo in uno spirito di collaborazione, attueranno questo piano. Siamo decisi a liberare la razza umana dalla tirannia della povertà e volgiamo guarire e proteggere il nostro pianeta.

6. Per una approfondita analisi sul BES si rimanda al sito: https://www4.istat.it/it/benessere-e-sostenibilità/misure-del-benessere 
Siamo determinati a intraprendere le azioni coraggiose e trasformative che sono urgentemente necessarie per portare il mondo su un sentiero di sostenibilità e resilienza. Mentre ci imbarchiamo in questo viaggio collettivo, ci impegniamo a far sì che nessuno sia lasciato indietro.

Queste dichiarazioni di principio potrebbero sembrare "troppo alte" da raggiungere per una loro effettiva realizzazione; ad una più attenta considerazione dei processi con i quali si intendono "aggredire" i diciassette obiettivi individuati, ci si rende conto che l'Agenda 2030 presenta non poche caratteristiche innovative rispetto a passate iniziative simili (anche se di minore portata). Questo progetto propone una visione integrata dei problemi e delle azioni da realizzare per conseguire lo sviluppo sostenibile, coinvolgendo tutti i Paesi a concorrere indipendentemente dalla loro situazione attuale. Ogni Paese deve declinare le proprie politiche economiche, ambientali e di welfare in coerenza con gli Obiettivi individuati dall'Agenda, al fine di rendere sistemico il percorso da compiere in vista di un risultato comune. Per rendere questo processo operativo occorre sviluppare un modello di sussidiarietà di tipo circolare, in grado di coinvolgere tutti i componenti della società quali sono le imprese, le pubbliche amministrazioni, le organizzazioni della società civile e i singoli cittadini. Solo attraverso una azione e una influenza reciproca di questi soggetti è possibile svolgere processi virtuosi in grado di rendere raggiungibili gli obiettivi individuati. Ciascun attore diviene primus inter pares, secondo una logica di orizzontalità che caratterizza i processi della postmodernità.

Tutto questo richiama anche un modo più maturo di vivere la responsabilità riferita a ciascun attore del sistema socio-economico; le imprese sono chiamate ad introdurre nei propri vettori strategici fattori di responsabilità sociale (e civile) nelle loro formule imprenditoriali; le organizzazioni della società civile (conosciute anche come organizzazioni del Terzo Settore o enti non-profit) devono trovare nuove forme di collaborazione e interazione sia con il sistema delle imprese, sia con il sistema delle amministrazioni pubbliche ai diversi livelli; queste ultime devono sviluppare sempre più azioni di coordinamento delle azioni territoriali e locali permettendo attività più focalizzate sugli obiettivi e ridu- 
cendo gli spazi della burocrazia; i cittadini, che sono anche consumatori, attraverso azioni che possono aiutare le aziende ad orientare in senso virtuoso i loro processi produttivi (il c.d. voto con il portafoglio) ${ }^{7}$.

\section{Cambio di paradigma}

Da quanto ora detto si comprende come sia sempre più necessario prefigurare un modello di mercato alternativo a quello attuale di stampo capitalistico al quale chiedere conto non solo dell'efficienza dei suoi risultati, ma pure della capacità di includere, almeno tendenzialmente, tutte le persone e di avvalorarle nelle loro diverse dimensioni. L'attuale modello di sviluppo ha portato molti benefici all'umanità; tuttavia non si può trascurare il fatto che le potenzialità insite in questo modello siano troppo spesso utilizzate per generare disuguaglianze piuttosto che per favorire la convergenza dei sentieri di sviluppo; più per incrementare livelli di utilità che per allargare gli spazi di felicità delle persone.

Già più di un secolo fa grande sociologo tedesco Max Weber sosteneva che l'economia non è una macchina, ma una costruzione sociale e traduce in consistenza materiale l'evoluzione spirituale e culturale di un popolo. A questa posizione concettuale gli faceva eco un grande statista ed economista italiano, Luigi Einaudi, affermando che: "Chi cerca rimedi economici a problemi economici è sulla falsa strada; la quale non può che condurre se non al precipizio. Il problema economico è l'aspetto e la conseguenza di un più ampio problema spirituale e morale» ${ }^{8}$.

Da queste affermazioni si comprende che il cambiamento di rotta può avvenire solo se sospinto anche da un impulso culturale e spirituale in grado di orientare i comportamenti umani verso gli obiettivi individuati. Ponendo mente alla storia delle civiltà, sempre i grandi cambiamenti hanno avuto alla loro base forti impulsi culturali e spirituali che hanno permesso il cambio di paradigma. In assenza di questa spinta, il rischio è quello che tutto rimanga confina-

7. Per un approfondimento sul tema si rimanda a L. Becchetti, Next. Una nuova economia è possibile, Albeggi Edizioni, Roma 2014.

8. L. Einaudi, Economia di concorrenza e capitalismo storico. La terza via fra i secoli XVIII e XIX, «Rivista di storia economica», giugno 1942. 
to nell'alveo dei buoni principi senza trasformarsi in vero agente di cambiamento.

Come bene argomenta Stefano Zamagni, un ordinamento sociale ed economico, per potersi sviluppare in maniera armonica ed equilibrata deve fare riferimento a tre principi regolativi. Il primo riguarda lo scambio di mercato fondato sul contratto e mediato dal pagamento di un prezzo, relativo al bene venduto e acquistato. Il secondo riguarda la redistribuzione pubblica della ricchezza prodotta, attraverso il sistema fiscale. Il terzo consiste nella reciprocità che si manifesta attraverso la gratuità e il dono, come espressione di fraternità ${ }^{9}$. Queste tre dimensioni devono convivere e alimentarsi a vicenda secondo uno sviluppo armonico e virtuoso. Il mercato propone il principio di efficienza nell'uso delle risorse impiegate per produrre; la redistribuzione delle risorse, fondata sull'autorità e sull'obbligo, propone il principio di equità. La reciprocità, intesa come dono reciproco, propone il consolidamento delle relazioni sociali, genera alleanza tra le persone, promuove la fiducia, la cooperazione, l'amicizia, la solidarietà, la libertà.

Questa impostazione è stata fatta propria dalla Dottrina Sociale della Chiesa nelle mirabili pagine dell'enciclica $\mathrm{Ca}$ ritas in Veritate (CV) di Papa Benedetto XVI, sulla cui linea di pensiero Papa Francesco ha proseguito con l'enciclica Laudato sii. In particolare nei numeri 37 e 38 della CV le due forme fondamentali con cui la riflessione classica, ripesa dalla tradizione cristiana, aveva interpretato la giustizia (commutativa e distributiva) vengono integrate da una nuova dimensione, la gratuità. Essa, declinabile in termini di dono, fraternità, solidarietà, non è confinabile in alcuni ambiti dell'attività umana ma deve essere presente in ogni attività economica e sociale, in quanto elemento costitutivo di veri rapporti umani integrali.

Leconomia, dunque, può avere una dimensione spirituale intesa in senso universale e non solo confessionale. «Ogni uomo e ogni donna cercano una realizzazione profonda, umana, interiore. In ogni uomo e donna esiste un 
"di più" misterioso. Un'economia che riduce la realtà e gli esseri umani (e viventi tutti) a una cifra non ha futuro» ${ }^{10}$.

Un interrogativo che può sorgere è se veramente una struttura economica con uno spirito nuovo, avente al centro la persona e che punti a uno sviluppo integrale, abbia realisticamente la forza di affermarsi su vasta scala e trionfare sull'attuale concezione economica. La risposta a questo quesito per ora non c'è; essendo un percorso ancora in divenire manca la certezza di una completa riuscita. Si può però affermare che tale processo è necessario, vedendo le conseguenze generate a livello globale del capitalismo odierno. Tale movimento non può che iniziare dal basso; molteplici sono gli esempi di realtà economiche che cercano di attuare un modello diverso di produzione e di organizzazione del lavoro. Bisogna partire da qui, da queste eccellenze di economia umana e aiutare a far si che tale modello si diffonda. Se dal particolare si possa passare all'universale, questo non è un movimento certo, non si può garantire che lavorare nel particolare porti in seguito anche a un cambiamento presso i grandi centri di potere economico e decisionali. Certamente tale compito, per quanto arduo, non deve spaventare, anzi deve essere portato avanti con passione ed entusiasmo, sia a livello di riflessione teorica sia a livello di attuazione pratica nel campo economico e sociale. Solo così sarà possibile tornare a coltivare e custodire il giardino prezioso che è la comunità umana. 

II.2. II contributo delle religioni

aA 



\section{Comunità umane, solidali, inclusive ed esperienza cristiana}

Antonio Sacco

L'esperienza cristiana non è mai indipendente da un'esperienza comunitaria e quest'ultima può ricevere dalla visione cristiana delle modalità di espressione che ne manifestino i tratti profondamente umani e non dis-umanizzanti o disumanizzati. Declinare la questione di comunità umane, solidali ed inclusive ${ }^{1}$ è centrale nella visione cristiana.

In questo intervento tentiamo di darne prima una ragione teologica ${ }^{2}$ e poi vogliamo riflettere sulla dimensione della prassi, della sua valenza etica in particolare dal punto di vista dell'etica teologica, per la realizzazione delle stesse.

\section{Fondazione}

Proponiamo tre aspetti teologici che vogliono riaffermare la centralità della socialità e della comunità nella visione cristiana.

1. Queste è la tematica estrapolata dall'Agenda Onu 2030 per lo sviluppo sostenibile (espressa nei punti 4, 10, 11 e 16 della stessa, cfr. https://unric.org/it/agenda-2030/ in data 7/4/2020). Ł̀ ispirazione per il tema della comunità che affronto in queste pagine.

2. Intendo la teologia secondo questa definizione di P. Coda: «la teologia [...] è una riflessione ed una chiarificazione intellettuale, metodica, critica e sistematica della conoscenza di Dio che si ha per fede, che è accolta a livello vitale ... e che porta a compimento quel "desiderio naturale di conoscere Dio" che è nell'uomo", P. Coda, Dio uno e trino, San Paolo, Cinisello Balsamo 1993, p. 14. 
È l'iniziativa rivelata di Dio che raduna un "popolo", lo costituisce e lo convoca. La struttura della rivelazione conduce a scoprire l'appello di Dio non rivolto semplicemente al singolo ma ad un popolo ${ }^{3}$. Un popolo, una comunità, che presenta il carattere dell'“unità nella diversità". Gli individui che lo compongono presentano notevoli differenziazioni, ma tutte in vista di un'origine comune e di una finalità comune: essa è sempre in Dio. Come ricorda Yves Congar:

sul piano antropologico ed etico, la legge fondamentale [di questo popolo] è di vivere gli uni per gli altri, di comunicarsi vicendevolmente ciò che si è ricevuto, di essere - per

Gesù ed in vista di Lui - ministri gli uni per gli altri, della grazia salutare. ${ }^{4}$

$\grave{E}$ in questo popolo che si comunica la vita cristiana sia temporalmente che spazialmente. La fede si riceve al suo interno: un legame con Dio che si esprime in un'unione realizzata nella mediazione delle persone e degli elementi sensibili. Il popolo di Dio è una realizzazione, sempre imperfetta, di comunità umane, solidali ed inclusive: esso è un tessuto di scambi e di rapporti reciproci ${ }^{5}$.

Ma quali sono le origini e le finalità dell'essere comunità, che cosa è chiamata a esprimere questa collettività?

Mi richiamo in particolare alle riflessioni di Stanley Hauerwas in merito ad un "popolo pacificato di Dio" il quale è tale perché ha accolto la proposta di un Regno pacificato ${ }^{6}$. Per quest'autore la vita cristiana possiede una dinamica inseparabile dalla dimensione comunitaria ed ecclesiale. L'insieme delle credenze cristiane dà vita ad una comunità che vive in fedeltà alla proposta del Dio dell'universo, rivelato, nella sua modalità più alta, in Cristo. La narrazione, il racconto di una trama narrativa, di una storia - che in questo

3. Il Concilio Vaticano II ha ribadito la centralità di pensare la chiesa come "popolo" di Dio nel capitolo secondo della Costituzione dogmatica Lumen Gentium.

4. Y. Congar, Un popolo messianico, Queriniana, Brescia 1976, p. 72.

5. La tradizione sociale cattolica, a partire dalla fine dell' 800 , ha riscoperto queste dimensioni propriamente per il piano del vivere sociale. L. Laberthonnière, uno dei protagonisti di quella stagione, ricordava che «una delle idee fondamentali del cattolicesimo è che tutto, nell'umanità si fa in collaborazione»: cit. in Y. Congar, Un popolo cit., p. 73.

6. S. Hauerwas, The Peaceble Kingdom, p. 16. Questa conclusione deriva dal fatto che la vita dei soggetti umani è sempre in rapporto ad una tradizione. 
Comunità umane, solidali, inclusive

caso è la storia della salvezza - è un aspetto intrinsecamente appartenente alla vicenda della comunità cristiana ${ }^{7}$. Infatti la vita cristiana si basa su una narrazione della storia fondativa, la narrazione della storia salvifica, che poi continua nelle intenzioni e nelle azioni storiche - e comunitarie - che nascono da quella narrazione.

Questa narrazione è centrale nella precisazione dell'evento Cristo: è dalla comprensione della vicenda di Cristo, che avviene non solo per i singoli ma in un insieme comunitario, che vengono precisate la funzione salvifica, la relazione con Dio e l'identità dell'individuo in relazione alla comunità. L'evento Cristo è di per sé affidato, già all'origine, ad un'esperienza comunitaria, quella dei discepoli, che diventano coloro a cui è consegnato l'insieme unitario della vita di Cristo, della sua Pasqua e della promessa futura di compimento, sorretti dalla presenza dello Spirito.

La dimensione comunitaria del cristianesimo ha un correlato: solo l'esistenza di realtà comunitarie che vivono l'esperienza etica cristiana può mostrare delle testimonianze credibili nella realizzazione della proposta cristiana. Non esiste alcuna “intellettualità" etica cristiana slegata da un'esperienza reale di popolo e di comunità anche con tutti i fallimenti e l'irraggiungibilità di una perfezione della stessa.

In questo senso possiamo affermare che la dimensione delle comunità umane, solidali ed inclusive non è fine a se stessa. Nella vita cristiana il popolo di Dio non è mai centrato su di sé, ma è portatore di un avvenire migliore che inerisce il destino collettivo delle persone ${ }^{8}$.

La Costituzione dogmatica, del Concilio Vaticano II, Lumen Gentium, ha ribadito che questo popolo ha la libertà e la dignità dei figli di Dio, ha per legge il precetto dell'amore di Cristo e per fine il Regno di Dio ${ }^{9}$. Queste caratteristiche sono solitamente comuni alle comunità umane - una condizione di vita, una legge ed una finalità - ma assumono uno specifico cristiano per il popolo di Dio.

Esiste il rischio che la solidarietà e l'inclusività, e per conseguenza anche la loro umanità, venga distorta parados-

7. Ivi, p. 34 .

8. Y. Congar, Un popolo cit., p. 86.

9. Cfr. Concilio Ecumenico Vaticano II, Costituzione dogmatica Lumen Gentium, p. 9. 
salmente proprio dalla specificità cristiana che viene contrapposta agli altri. Questo accade quando ci si considera destinatari esclusivi della libertà, dignità e dell'amore del Regno. Ma se il popolo di Dio, e la sua espressione comunitaria, vuole rimanere all'altezza delle caratteristiche che sono un dono di Dio, non può escludere gli altri piuttosto deve sempre diventare includente.

All'opposto questa santità ed elezione significano il tentativo di non escludere gli altri, ma vivere come un popolo cristiano che nella storia del mondo crea unità tra popoli, nazioni ed istituzioni. Per l'argomentazione che stiamo sviluppando un popolo che genera in senso universale comunità umane, solidali ed includenti. Un popolo messianico che esiste per l'umanità intera ${ }^{10}$. Ovvero portatore di una speranza, di una liberazione e di un avvenire migliore che riguardano il destino integrale delle persone, in particolare nella loro espressione sociale e comunitaria.

\section{b) Relazione trinitaria e relazionalità umane}

Un secondo aspetto che vede il Dio cristiano come un Dio, in se stesso, legato alla relazione, è centrale per capire lo sguardo sull'esperienza comunitaria umana ${ }^{11}$.

La scoperta del "mistero" di Dio, lo sguardo teologico sulla sua natura a partire dalla rivelazione, ci offre una proposta di indole "comunitaria": la prospettiva del Dio cristiano come Trinità. La relazione trinitaria si è resa presente in un punto culminante della storia della salvezza: in Gesù Cristo ${ }^{12}$; è in particolare nel momento fontale della Pasqua dove Gesù viene consegnato per amore, dal Padre; Gesù si consegna al Padre; il Padre e Gesù consegnano lo Spi-

10. Y. Congar, Un popolo cit., p. 83.

11. Questo approccio ha radici lontane nella teologia, ad esempio sistematizzato negli scritti degli anni '40 del secolo scorso di A. Geck. (cit. in G. Thils, Teologia e realtà sociale, 1967 , or. franc. $1958^{1}$; la prima versione è stata rifusa in italiano, 1967), p. 77. Esso consiste nel considerare la comunità di vita tra Padre, Figlio e Spirito Santo come paradigma della vita umana in comunità. Anche R. Guardini ha uno scritto, all'inizio del secolo scorso, che rimane centrale su questo tema, cfr. R. Guardini, Il senso del dogma della SS. Trinità e la vita morale della comunità, in Id., Scritti Politici, in M. Nicoletti (a cura di), Opera omnia. 6, Morcelliana, Brescia 2005 (or. ted. 1916).

12. In questo senso nel cristianesimo fede cristologica è allo stesso tempo fede trinitaria, cfr. P. Coda, Dio uno e trino, San Paolo, Cinisello Balsamo 1993, p. 8. 
Comunità umane, solidali,

inclusive

rito Santo ${ }^{13}$. Questo dinamismo trinitario è figura di ogni dimensione relazionale ed in senso allargato comunitaria.

Il monoteismo ha dovuto subire l'accusa di una difficoltà a far spazio all'alterità, ed alla reciprocità, quali aspetti della molteplicità del vitale, accusa che viene proposta ad esempio da Nietzsche. Questo tema "relazionale", che riguarda l' "in sé" di Dio, contrasta il fatto che l'idea di un Dio uno ed unico sarebbe di per sé la negazione del valore dell'alterità e della dimensione plurale ${ }^{14}$. Questa difficoltà viene evitata se si considera la Trinità come la figura cristiana, e cristologica, del monoteismo stesso. Essa non riporta ad un Dio solitario bensì l'idea del Dio unico, di per sé, vuole escludere una pluralità di falsi dei.

La dimensione trinitaria emerge dalla rivelazione: nel cristianesimo l'unicità di Dio non è qualcosa di "ontologico" a priori, che può essere compresa dall'esterno, ovvero dall'esterno dell'evento Cristo. Come afferma Piero Coda:

il monoteismo trinitario non è semplicemente esclusivo: nel senso che afferma l'assolutezza sovrana di Dio [...] ma è insieme inclusivo [...] si apre e partecipa del suo stesso mistero attraverso la rivelazione di Gesù Cristo $[\ldots]$ ed il dono dello Spirito alle creature..$^{15}$

L'unità di Dio si mostra, nella rivelazione, come un'unità che è principio, ma anche finalità, delle relazioni d'amore delle tre persone divine.

La relazione trinitaria diventa figura per la prospettiva relazionale umana ${ }^{16}$. Essa è avvertita direttamente dal credente, e si apre all'universale dell'umano, che incontra in essa una figura per la relazionalità in senso ampio.

Quello che l'uomo percepisce come già appartenente alla sua natura - ovvero la dimensione sociale, comunitaria e relazionale - non ha già in sé una piena realizzazione. Piuttosto guarda alla pienezza che si individua nella relazione

13. P. Coda, Dio, Dizionario di Teologia 2002, p. 429.

14. Ivi, p. 440.

15. Ivi, p. 442.

16. Non affronto direttamente il tema della comunità ecclesiale dove l'evento pasquale, che ha la sua origine trinitaria, diventa attuazione di un disegno - di Dio - sull'umanità intera attraverso la Chiesa, che è in Cristo come sacramento, ovvero segno e strumento della comunione con Dio e dell'unità dell'umano, secondo la nota definizione del Concilio Vaticano II, nella Costituzione dogmatica Lumen Gentium, n. 1. 
trinitaria, la quale non presenta alcuna oscurità nell'amo$\mathrm{re}^{17}$, come fine da realizzare in ogni dimensione relazionale e comunitaria, diventando la realtà che le comunità dei credenti, ed in universale degli uomini, debbono tradurre in un percorso che si attua nel tempo e nella storia. Potremo dire che è un fine ultimo, nel senso che indirizza tutti i fini sociali umani. Prima comunità solidale ed inclusiva è quella divina, figura di ogni comunità umana.

Tuttavia non si tratta di un modello deduttivo, una sorta di idealismo e di adeguazione ontologica, ma di un radicarsi della Trinità nella storia. Questo avviene sia nell'origine dell'acquisizione cristiana dell'esperienza trinitaria come esperienza storica ${ }^{18}$, che nell'attuazione sempre nella storia, dell'intera vita cristiana. Le comunità inclusive si danno nel tempo e non nell'astratto dell'idea ${ }^{19}$. Guardare alla relazione trinitaria come figura della relazione umana comunitaria non deve mai svalutare quest'ultima, che ha già valore proprio in se stessa, ma vuole spingerla verso un fine che ne dica la pienezza, che l'arricchisca e ne faccia crescere l'aspetto profondo nella direzione solidale ed inclusiva.

In sintesi la dimensione trinitaria che si riverbera, si irradia, nella comunione dei soggetti propone un'antropologia sociale di comunione, inconciliabile con elementi quali il potere esclusivo, il possesso egoistico, e tutte le condizioni che distruggono solidarietà ed inclusione. Questa antropologia

17. Nella Costituzione pastorale, del Concilio Vaticano II, Gaudium et Spes, al n. 24, viene citato il passo di Gv 17,pp. 21-23: «affinché tutti siano una cosa sola come anche noi siamo una cosa sola, o Padre», che suggerisce una similitudine tra l'unione delle persone divine e l'unione dei figli di Dio nella verità e nella carità. Il testo ricorda tra l'altro che questa similitudine è un orizzonte complesso per la sola razionalità umana. Si potrebbe altrimenti affermare che l'amore è il modo di attuazione - anche quello che è centrale, l'essenza - dell'unità tra gli uomini. Amore che nasce dall'amore in Dio, e che incontra il suo contrario nella divisione.

18. Cfr. K. Rahner, H. Vorgrimler, Trinità, in Id., Dizionario di Teologia, Herder - Morcelliana, Brescia 1968, p. 739: «Dio nella sua auto-partecipazione alla creatura si è comunicato in maniera tale che la Trinità immanente è la Trinità economica. [...] quale mistero avvicinatosi a noi si chiama nella sua assoluta originarietà Padre; quale principio che agisce nella storia si chiama Figlio; quale principio donato a noi e accettato si chiama Spirito Santo", dove il quale di questa definizione sancisce il donarsi di Dio in sé, una triplicità che riguarda Dio in sé.

19. Cfr. Francesco, Lettera apostolica Evangelii Gaudium, nn. 231-233, dove si sottolinea che «la realtà è più importante dell'idea». Il n. 233 ricorda «il criterio di realtà, di una Parola già incarnata e che sempre tenta di incarnarsi» propone, per il legame tra Trinità e comunità, un prendere forma nella storia effettiva. 
Comunità umane, solidali, inclusive sociale comunionale rifiuta privilegi: è fondata sulla libertà, si realizza nel servizio reciproco ${ }^{20}$.

c) Tradizione cristiana e promozione delle comunità

La tradizione cristiana, che è l'estensione temporale dell'esperienza salvifica di Gesù, ha sempre riconosciuto una centralità, una costante, alla socialità ed alla comunità nella sua visione antropologica, sia in maniera teorica che pratica ${ }^{21}$.

Il cristianesimo propone come ideale quello di una comunione tra soggetti fondata in Dio. Ma accanto a quest'aspetto più “ontologico", postula anche un aspetto concreto, una relazione con Dio, meglio un'espressione di questa relazione, che si esprime in maniera non solamente individuale, ma deve farlo in una modalità comune e comunitaria ${ }^{22}$.

Un'antropologia, ed una prassi, cristiana integrale manifesta la sua attenzione alla dimensione sociale e comunitaria. Redenzione e salvezza sono opera di unità e di comunione - mentre il peccato può essere ridetto proprio con la categoria della divisione tra gli individui - riguardanti i soggetti: la redenzione di quell'uomo che è membro di una famiglia, di una comunità civile, di una "società professionale" e simili ${ }^{23}$.

Se l'individualismo dell'epoca moderna, anche nel cristianesimo, ha messo in rilievo il "verticale" del rapporto con Dio, il contemporaneo ha recuperato l'aspetto "orizzontale": unione degli uomini tra loro e con Dio, unione che si realizza attraverso Cristo ${ }^{24}$. Ma questo "orizzontale" si esprime nella concretezza del comunitario dell'esperienza umana: esiste un carattere collettivo della salvezza ${ }^{25}$.

20. I. Sanna, Chiamati per nome: antropologia teologica, Edizioni Paoline, Milano 2007, p. 163.

21. Nel corso della storia della tradizione cristiana si è presentato anche un oscuramento di questa centralità, in particolare nell'epoca moderna, dove ci si è molto focalizzati sulla salvezza individuale.

22. G. Thils, Teologia e realtà sociale, p. 39.

23. Ivi, p. 26.

24. Cfr. 2Cor 1,4-5a: in questo passo l'unione relazionale tra Paolo ed i Corinzi avviene in una compartecipazione alla consolazione di Cristo.

25. Sempre nell'ottica della nota precedente, Thils, citando M. Goguel, sottolinea che se nelle lettere paoline c'è una salvezza individuale, è presente anche un'unione collettiva a Cristo. Essa viene esplicitata con forza nella lettera agli Efesini come l'unione del capo con il corpo: cfr., G. Thils, Teologia e realtà sociale, p. 27. 
Questa espressione comunitaria della vita cristiana inoltre rimanda ad un tema centrale: il contrasto del pensare il cristianesimo come "disprezzo del mondo", di cui spesso è stato accusato il cristianesimo. La dimensione della creazione riporta alla bontà delle cose e delle strutture umane, nella prospettiva dell'affidamento collettivo della creazione all'umanità da parte di Dio ${ }^{26}$. La creazione è un'espressione dell'agire salvifico di Dio, che ha il suo culmine nella prospettiva dell'incarnazione, nell'agire di Gesù in mezzo agli uomini, creazione a cui appartiene già la relazionalità quale parte elementare della dimensione comunitaria. Significa un interesse per una spiritualità cristiana inserita nel mondo e che non lo denigra. Il primato del soprannaturale rende più concreto l'impegno ed il servizio dell'uomo nel mondo ${ }^{27}$, anche nel favorire l'esistenza di dimensioni comunitarie più autentiche ed aperte alla solidarietà ed all'inclusione. La dimensione dell'amore teologale indirizza la vita cristiana e deve anche inserirsi nella vita della comunità umana, della famiglia e della società.

La centralità dell'ottica comunitaria, nell'esperienza cristiana, è stata sottolineata e sintetizzata dalle pagine della costituzione pastorale Gaudium et Spes, Sulla chiesa nel mondo contemporaneo (GS). Nel secondo capitolo della prima parte della stessa (GS, nn. 23-32), che ha come titolo La comunità degli uomini, vengono messe in rilievo le caratteristiche centrali della comunità.

Già in precedenza GS 12 ricordava che «l'uomo è un essere sociale e senza rapporti con gli altri non può vivere ed esplicare le sue doti». Questa non è un'idea solamente funzionalistica, ma un aspetto che caratterizza più profondamente la vita umana.

In particolare la sviluppo della persona e lo sviluppo della società sono strettamente interdipendenti (GS 25): non è possibile pensare un soggetto non congiunto alla dimensione sociale, poiché i legami della stessa sono necessari al perfezionamento dell'uomo. Sono ad esempio: famiglia, comunità politica, maggiormente dipendenti dalla stessa

26. Per le questioni della separazione tra Dio ed il mondo e le conseguenze del disprezzo del reale, cfr. G. Colzani, Il rapporto con il mondo, «La Scuola Cattolica», 5 (set.-ott.) - 1993, pp. 665-669.

27. Ivi, p. 668 . 
Comunità umane, solidali, inclusive

natura umana; altri, come le socialità intermedie, più legati alla libera volontà di associarsi. Il concilio ribadisce come le persone possono essere deviate dal contesto sociale nel raggiungere il bene. Viceversa ricorda che anche superbia ed egoismo pervertono l'ambiente sociale.

Quest'affermazione di GS indica una duplicità. Da un lato l'ambiente comunitario influisce sui soggetti, è la prospettiva di un certo determinismo, strutturalismo, sociale che rischia di elidere il singolo, di condizionare la sua libertà e volontà. Dall'altro però sempre gli individui, facendo il male, possono influenzare il contesto comunitario e sociale. In questo senso, nelle prassi, la questione virtuosa emerge come primaria.

Il concilio pone l'obbiettivo, il fine, dell'esistenza comunitaria nel bene comune (GS 26). In fondo non è che una modalità per esprimere gli scopi della vita comunitaria: l'insieme delle condizioni della vita sociale che permettono ai gruppi ed ai singoli di raggiungere la propria perfezione più pienamente. Una comunità deve essere solidale perché è sempre il bene di tutti che deve essere oggetto di attenzione, ma allo stesso tempo includere tutti i soggetti, non sopprimendo bisogni e necessità di ciascuno, integrandoli nella prospettiva del comune.

In questo senso GS 25 ricorda che:

Lordine sociale pertanto e il suo progresso debbono sempre lasciar prevalere il bene delle persone, giacché nell'ordinare le cose ci si deve adeguare all'ordine delle persone e non il contrario, secondo quanto suggerisce il Signore stesso quando dice che il sabato è fatto per l'uomo e non l'uomo per il sabato.

In fondo queste affermazioni possono essere programmatiche per realizzare delle comunità inclusive e solidali. Perché queste qualità non possono essere realizzate senza un'attenzione al primato personale rispetto alle visioni funzionali e strumentali. La persona non è mai solo un mezzo o una parte rispetto al tutto. La sua sub-ordinazione è solo per i compiti che gli sono assegnati per il bene comune, dell'insieme.

Allo stesso tempro inclusione e solidarietà nell'agire comunitario sono in vista del bene comune. I singoli si indirizzano nel loro agire alla realizzazione di un bene collettivo: 
siamo in una "unità di ordine" - una serie di prassi ordinate, indirizzate, a qualcosa -, di azione e di fine dei membri di una comunità-società, dove l'insieme non ha esistenza specifica al di sopra delle parti ma è un'unità di ordine, di fine $^{28}$.

L'apporto della GS, nella sintesi dei paragrafi proposti, afferma dunque il valore centrale dell'esperienza comunitaria per la vita umana, proponendola come imprescindibile dalla realizzazione della stessa umanità.

Queste tre prospettive teologali - una salvezza donata ad un popolo, relazione trinitaria e relazionalità umana, tradizione cristiana e promozione della comunità - hanno mostrato una sorta di fondamento, non esaustivo, del rapporto tra riflessione cristiana e modalità dell'esperienza comunitaria. La dimensione comunitaria non è qualcosa di aggiunto e di accessorio alla vita cristiana, ma appartiene alle sue dimensioni più profonde.

Ora guardiamo alla realizzazione, nella prassi, della forma comunitaria. La prospettiva etica, la ricerca del bene presente della vita comunitaria, propone una riflessione sulle caratteristiche delle comunità, sulle loro qualità e caratteri, che se presenti dicono la bontà della loro esistenza.

Detto altrimenti quali virtù sociali debbono avere delle comunità per essere maggiormente umane, inclusive e solidali?

Il paragrafo che segue tenterà di offrire la risposta di una delle prospettive dell'etica cristiana.

\section{Prassi}

a) Comunità e vita etica

Non è sufficiente affermare una convivenza, una relazionalità generica tra i soggetti, per definire la bontà della comunità, ma occorre precisarne la qualità: una comunità deve essere sempre coinvolta in un dinamismo di crescita per realizzare il bene. Nelle nostre argomentazioni per diventare solidale ed inclusiva. La domanda è come rendere possibili, nella realtà, comunità solidali ed inclusive e non individualistiche ed escludenti? 
Comunità umane, solidali, inclusive

Una delle possibili vie per esplicitare questa differenza è la via dell'etica delle virtù, una delle principali teorie etiche anche in senso cristiano ${ }^{29}$.

La riflessione etica esprime nelle teorie etiche la ricerca del bene vissuto dai soggetti nelle prassi e nelle esperienze sociali.

Un primo concetto fondativo emerge dall'etica classica: «solo dei cittadini virtuosi fanno buona la polis» ${ }^{30}$. Affermare che una comunità diventa solidale ed inclusiva significa ragionare sull'acquisizione delle virtù da parte dei singoli. In particolare esiste una virtù cristiana che è specificatamente comunitaria: essa è la carità o amore (agàpe). Ad essa associamo una virtù che è universalmente umana e nondimeno centrale nel discorso cristiano: la giustizia.

Proponiamo, nelle pagine seguenti, una delle possibili ricomprensioni delle virtù nel discorso etico contemporaneo ad opera di Alasdair MacIntyre ed in seguito legittimiamo l'insieme della valenza di giustizia e carità per la vita delle comunità.

b) Le pratiche

Una visione etica che proponga solo dei valori o delle norme individuali, per quanto espressive del bene, rischia di favorire una concezione ideale che in seguito non riesce a incidere sulla realtà. Non è sufficiente indicare una serie di valori e di norme a cui i soggetti si dovrebbero adeguare senza tenere conto della strutturazione comunitaria. Per rendere meno astratto il discorso sulle virtù nella comunità, proponiamo uno schema che vede l'impegno etico in relazione alla vita degli organismi collettivi.

Qui ci rifacciamo alle teorie di MacIntyre sulla questione delle pratiche che rendono puntuale, attualizzano, il discorso etico applicato alla comunità ${ }^{31}$.

Le pratiche sono attività comunitarie, sono un'attività cooperativa umana per realizzare un determinato scopo,

29. L'etica teologica è sempre un insieme di razionalità e di apporto della fede alla razionalità medesima. Uno specifico cristiano dell'etica include la razionalità etica e allo stesso tempo arricchisce questa razionalità di nuove prospettive che sorgono dal dato teologico. 30. È una delle tesi del pensiero di Platone, ad esempio.

31. A. MacIntyre, Dopo la virtù, Armando, Roma 2007, p. 232. Per uno sguardo sintetico sulla proposta di MacIntyre: A. Sacco, La giustizia come virtù, Effatà, Cantalupa (To) 2017, pp. 15-34; per le pratiche: ivi, pp. 193-196. 
in collaborazione con altri soggetti. Esse riguardano tutti gli ambiti comunitari: il gioco, le arti, le scienze, la costruzione della vita domestica, la ricerca scientifica compresa quella teologica e simili. Come sottoinsiemi ne sono parte le istituzioni e i gruppi stessi che le compongono. La loro caratteristica fondativa è la dimensione collaborativa. Ad esempio, uno studente che studia da solo non esercita una pratica, mentre la esercita un gruppo di studenti che prepara un seminario.

Una seconda caratteristica è la distinzione tra beni esterni ed interni alle pratiche.

I primi escludono e sono contrari ai dinamismi solidali, esempi sono il potere come affermazione di sé, il prestigio fine a se stesso, il guadagno senza limiti e simili.

Interni sono quelli per cui una certa attività è finalizzata, ad esempio l'università per il sapere, il parlamento per emettere leggi giuste, gli ospedali per la cura dei malati e così via. Gli scopi interni sono quelli per cui una certa pratica nasce ed ha un senso proprio. Chi prende parte alla sua attuazione lo fa primariamente per uno scopo interno alla stessa. Si partecipa ad un corso di studio per l'accrescimento culturale che il corso offre, non solamente per il prestigio che quel corso donerà al curriculum di studio. Il primo sarà un bene interno alla pratica, il secondo esterno.

I valori interni possono venire raggiunti in una pluralità di modi, ma il valore conseguito sarà a favore di tutti, il valore del bene interno è posseduto dall'intera comunità che partecipa alla pratica ${ }^{32}$. Se ad esempio nelle pratiche universitarie si cerca un arricchimento legato alla crescita personale o della ricerca si persegue un bene interno, se invece si vogliono ottenere dei beni esterni - prestigio, potere, evidenza, ricchezza e simili - essi saranno contrari al senso ed ai fini della pratica stessa.

La tesi centrale del filosofo scozzese è che per raggiungere dei beni interni ad una pratica occorra l'acquisizione delle virtù ${ }^{33}$.

32. A. MacIntyre, Dopo la virtù cit., p. 235.

33. Viene a questo proposito recuperata ed introdotta una prospettiva classica dell'etica, quella della dimensione virtuosa che è di radice aristotelica e nella tradizione cristiana è stata ripensata, ed arricchita teologicamente, da S. Tommaso D’Aquino. La Seconda parte della Summa Teologiae ripropone un'etica delle virtù come indirizzo fondativo dell'etica 
Comunità umane, solidali,

inclusive

Esse sono delle disposizioni interiori che permettono di raggiungere il bene. Inoltre diventano dei fini da perseguire: permettono un compimento umano, offrono una pienezza all'agire in vista di dare un senso all'esistenza ${ }^{34}$. Le virtù esprimono un'assimilazione al bene da parte del soggetto che desidera radicarsi in quello che è la motivazione interna di una pratica.

Le virtù sono un elemento fondativo delle pratiche. Infatti poiché queste ultime possano riuscire come impresa collaborativa, i soggetti coinvolti in essa devono esercitare le virtù: giustizia, veridicità, fortezza e simili ${ }^{35}$. Le comunità solidali ed inclusive sono possibili se i soggetti sono virtuosi. I desideri di successo e di affermazione sono desideri leciti per i soggetti tuttavia solo se sono compatibili con le virtù della giustizia, dell'onestà, del rispetto dell'altro, della veridicità e simili che tutelano i beni interni delle pratiche.

Se si amplia questo discorso delle pratiche alla globalità di una vita umana vedremo che solo alcuni fini donano una pienezza che è legata al bene autentico, alla vita buona. Senza le virtù - ad esempio la giustizia - la ricerca del bene come fine che dona unità alla vita viene meno.

Un'ultima considerazione vede una reciprocità tra comunità e soggetti. L'appartenenza di questi ultimi a comunità inclusive e solidali da un lato rafforza le virtù individuali, dall'altro permette che le comunità esistano in quella modalità.

Infatti le virtù dei singoli si rafforzano, e si mantengono, solo se trovano dei "luoghi umani" che ne permettono la crescita e l'espressione creativa. Senza delle "pratiche" co-

cristiana. Cfr. G. Abbà, Quale Impostazione per la filosofia morale? Ricerche di filosofia morale, LAS, 1996, pp. 57-68. L'autore citato è il maggior esponente dell'etica delle virtù in Italia. 34. Occorre proporre un'osservazione di fondo. L'etica è relata in questo caso alla visione dell'umano che si vuole raggiungere: essa dona un compimento all'umano, ne è la realizzazione propria. Questa è una concezione teleologale, in senso classico, dell'etica, dove l'indirizzo dell'agire è in vista del fine ultimo, la felicità-beatitudine. In una prospettiva non teologale questo fine è nella "vita buona", ovvero nell'insieme dei beni che danno pienezza al soggetto ed invece nell'arricchimento teologico è sempre in Dio stesso, inteso come fine inclusivo della prospettiva morale. Le virtù sono mezzi intrinseci al fine della vita buona, ovvero lo realizzano di per sé. La vita buona consiste nell'insieme della realizzazione di beni che non son funzionali ad altri scopi (beni utili) ma hanno un valore di per sé (beni onesti)). Qui non si intendono le virtù come delle attitudini o dei mezzi per realizzazione di una produzione (skills).

35. Cfr. G. Abbà, Felicità, Vita buona e Virtù, LAS, Roma 1995, p. 132. 
munitariamente virtuose sarà molto difficile che il singolo riesca a perseverare nella ricerca dei beni interni anche se possiede dei fini virtuosi. Questi infatti vengono messi a rischio proprio dal fatto di non poter essere attuati pienamente, anzi contrastati nell'agire collettivo ${ }^{36}$.

Qui certamente l'orizzonte motivazionale del singolo formato dalla fede, dalle virtù, dai fini etici e la sua struttura "di sostegno" - famiglia, amicizie, chiese ed altre socialità intermedie - possono risultare decisive per l'insieme delle pratiche. Le virtù e le strutture personali sono determinanti per il raggiungimento del bene, e dei beni che lo esprimono, nelle situazioni dove le pratiche comunitarie sono complessivamente sbilanciate sui beni esterni.

Nel paragrafo che segue ci soffermiamo in particolare su due virtù che si uniscono in un apporto fondamentale alla costruzione della comunità. Analizzeremo la valenza della giustizia, come virtù fondamentale dell'etica "umana” e cristiana, ma in vista di un arricchimento della stessa grazie all'apporto di uno specifico cristiano che è la carità.

\section{c) Giustizia e Carità}

Da un lato, la virtù centrale della prassi comunitaria sarà la giustizia, essa è la virtù che riguarda la relazione con gli altri.

Dall'altro, nella visione cristiana, avremo bisogno sempre della carità intesa come la presenza stessa dell'amore di Dio che tocca la vita umana.

\section{- Giustizia}

La giustizia permette un indirizzo al bene autentico dei soggetti ${ }^{37}$. Senza la giustizia non si possono assolvere i doveri verso la comunità, nei ruoli sociali ricoperti dai singoli. Allo stesso modo neanche i doveri privati, all'interno delle strutture familiari o amicali, possono essere compiuti. Senza di essa mancherebbe una capacità di discernere quali sono i beni autentici dei soggetti. Per esistere le comunità solidali

36. Le comunità ideali sono quelle che riusciranno a far sì che non esista un contrasto tra fini personali e condivisi, di modo che i soggetti si sentano pienamente in grado di realizzare il bene proprio realizzando anche i fini comunitari. Questa è, ad esempio, la funzione per Aristotele della polis.

37. A. Sacco, La giustizia, p. 154 e pp. 156-166. 
Comunità umane, solidali,

inclusive

ed inclusive debbono possedere, come virtù dei soggetti che appartengono ad esse, la giustizia.

Per caratterizzare la giustizia la esprimiamo con due principi che sono esplicitazioni della razionalità pratica, la razionalità che permette di cogliere il bene:

- il principio di eguaglianza: "dare all'altro quello che gli è dovuto" 38 . Esso è un principio che si struttura in una serie di principi derivati e ancora generali, che ne permettono una specificazione dall'universale al particolare: restituire i prestiti, occuparsi di chi ha bisogno, non dimenticare i più deboli, essere leali allo Stato e simili ${ }^{39}$.

- il principio di non maleficenza: "tutto quello che può fare del male deve essere evitato" 40 .

La presenza della giustizia, di soggetti giusti, non permette di considerare in modo indifferente azioni che causano un male ad altri: vanno sempre evitate.

I principi di giustizia tutelano i soggetti all'interno di una comunità nei rapporti reciproci.

La presenza - o assenza - della giustizia si coglie proprio dalla declinazione di questi principi nelle differenti situazioni e circostanze. Non si tratta però di un metodo deduttivo ma "analogo": è dal confronto tra azioni giuste, in contesti differenti, che si può cogliere - nella constatazione della presenza dei principi ed anche nel discernimento delle circostanze legate alle situazioni reali - la realtà della giustizia.

La questione della giustizia come virtù si esplicita in tre dimensioni.

La prima è legata ai principi in relazione ai fini, agli scopi virtuosi che si vogliono raggiungere: i principi sono i fini dei soggetti. Essi vorranno nel loro agire personale - e collettivo - rendere presente la giustizia: la loro "intenzionalità" è guidata dai principi di giustizia.

La seconda vede la virtù di giustizia come una disposizione delle capacità operative del soggetto (habitus) che apporta una trasformazione in vista dell'operare il bene e che

38. Nella tradizione tommasiana: S Th II II 57.

39. Questi principi riguardano diversi aspetti della giustizia: ad esempio reciprocità giusta tra i soggetti (giustizia commutativa), giustizia degli insiemi sociali verso il singolo (distributiva), dei singoli in relazione al collettivo (sociale).

40. Nella tradizione tommasiana: S Th I II 100.5 Questo principio è in parte simile alla regola d'oro detta in termini negativi: non fare il male che non vorresti foste fatto a te. 
tiene in conto l'interezza della persona: un cambiamento in vista del bene del "conoscere praticamente", del volere e delle espressioni passionali. Significa che il soggetto acquisisce un'ottica personale che gli permette di perseguire più profondamente i principi di giustizia come strutturanti la sua persona.

La terza riguarda l'identificazione della virtù attraverso l'insieme delle azioni giuste che il soggetto compie e che sono in un'ottica di giudizio oggettivo, ovvero hanno un riscontro nella realtà, nel loro essere giuste di per sé e negli effetti prodotti ${ }^{41}$.

Infine la via virtuosa cristiana e la giustizia saranno in parte uguali ed in parte differenti dalla via virtuosa e dalla giustizia pensate solo umanamente. Le virtù si indirizzeranno ad un compimento che è ugualmente morale, ma allo stesso tempo, nell'esperienza cristiana, troveranno un fine più grande nell'indirizzo a Dio stesso ${ }^{42}$.

Questo compimento è rappresentato da una categoria teologica che è la carità.

- Carità

La carità, nella visione teologale, è l'amore di Dio, il Dio che ama l'uomo. È anche un amore con una declinazione che potremo definire antropologica: è un amore presente nella vita dell'uomo e che si amplia come risposta verso l'amore per il prossimo, un amore che si diffonde passando da Dio all'uomo, e che si comunica da soggetto a soggetto. In questo senso la carità è una virtù teologale perché è un dono di Dio che, in quanto virtù, trasforma il soggetto e si radica in lui.

L'amore cristiano, la carità, esprime un'attenzione alle persone nella loro particolarità ed unicità e tocca tutti gli aspetti già in vista del bene indirizzandolo direttamente a Dio $^{43}$. Essa prevede sempre un'oblatività - un donarsi ed un aiuto reciproco - tra soggetti che si relazionano tra loro.

41. Quest'ultima considerazione propone un raffronto tra la teoria etica deontologica e quella consequenzialista.

42. Non affronto la questione delle dimensioni implicite dell'azione giusta come indirizzate esse stesse a Dio. Questa è la tematica dell'implicito teologale che è una delle grandi acquisizioni della svolta antropologica in teologia.

43. Nella tradizione tommasiana si dirà che la carità è forma di tutte le virtù: cfr. S Th II II 23,8 . 
Comunità umane, solidali, inclusive

Loperare della carità è un innesto che dona frutti nuovi alla giustizia. La giustizia viene espressa con dei principi, ma in questo caso sarà un principio nuovo che la indirizza. Esso è definito come:

- principio di gratuità: "tutto quello che eccede, che è oltre, il dovuto". Possiamo definirlo come il "supererogatorio", quelle azioni che sono ammirate perché vogliono realizzare il bene ad ogni costo. ${ }^{44}$

La carità ridefinisce la giustizia integrandone in essa il concetto di "oltre il dovuto".

Tra le due virtù nasce una circolarità: la carità non si presenta senza la giustizia, quest'ultima è intrinseca alla prima. Senza la giustizia non siamo in grado di dare una consistenza minimale alla carità, essa rimane ideale e spesso rischia di essere un discorso che non entra nel cambiamento reale delle situazioni.

Viceversa la giustizia rischia di presentarsi solamente come un aspetto oggettivo, impersonale, che non presta attenzione alle persone, ai singoli ed alle loro situazioni. La carità conduce la giustizia all'"oltre il dovuto" che realizza un amore autentico.

In particolare, come afferma David Hollenbach, l'amore cristiano rafforza la reciprocità (mutuality) come caratteristica che appartiene propriamente alla giustizia ${ }^{45}$. Detto altrimenti la tradizione del pensiero sociale cattolico ha dato forma alla giustizia nell'amore. Quest'ultimo è una parte costitutiva della giustizia e la giustizia realizzata è una forma di amore.

Da un lato si ricollega l'amore all'uguaglianza che crea reciprocità. L'espressione concreta dell'amore è il fatto che i soggetti non rimangano chiusi nelle loro prospettive individualistiche ma crescono nella ricerca del bene reciproco perché sentono una compartecipazione ad una comune umanità. Dall'altro le motivazioni profonde per la giustizia si ricollegano alla fede: è l'amore di Dio verso l'umanità, che si è manifestato in maniera esemplare nel mistero pasquale,

44. Sono spesso azioni che non riescono a portare a compimento lo scopo che si prefiggono, come tentare di salvare la vita ad una persona anche se non si hanno strumenti adatti per farlo.

45. D. Hollenbach, Justice, peace, and human rights: American Catholic social ethics in a pluralistic world, Crossroad, New York 1988, p. 18. 
che diventa motivazione del soggetto per essere giusto e fare la giustizia. È la prospettiva dell'amore cristiano che forma la giustizia. La motivazione che anima la giustizia è l'amore cristiano al prossimo che ha come sorgente l'amore di Dio verso questo prossimo.

In conclusione comunità solidali ed inclusive si devono ricondurre all'affermazione della giustizia e, in una prospettiva teologica, della carità.

\section{Prospettive sintetiche}

In queste pagine abbiamo cercato di compiere due passaggi. Abbiamo indagato delle prospettive fondative per il concetto di comunità inclusive e solidali a partire dai fondamenti della riflessione teologica. L'idea del popolo di pacificato di Dio, del legame tra la Trinità e le relazioni e della centralità della vita comunitaria nella vita umana per il cristianesimo, hanno permesso di comprendere delle ragioni fondative nel discorso cristiano sulle comunità inclusive e solidali.

Questi fondamenti sono un bagaglio di ragioni da parte dei cristiani - concretamente con tutti coloro che, anche da altre tradizioni ed indirizzi, si sentono interpellati dalle stesse - per costruire un futuro meno individuale e competitivo ma più solidale e comunitario.

Abbiamo poi offerto alcune prospettive etiche in merito alla prassi, nella strutturazione delle "pratiche", per il concreto realizzarsi delle comunità. Introducendo la proposta delle virtù si è voluto sottolineare una polarità tra individuo e comunità ${ }^{46}$, che le virtù, nel riferirsi all'individuo, ampliano poi per il bene collettivo. In particolare la relazione tra giustizia e carità esprime la via etica cristiana per la vita comune. Nondimeno diventa proposta virtuosa che viene offerta a ogni soggetto per una visione sociale della vita umana che conduca a comunità solidali ed inclusive. 
Roberta Aluffi

Individuare il punto di vista islamico su una qualsivoglia questione è sempre una sfida, considerata l'assenza di un'unica autorità religiosa, e al contempo la molteplicità di tradizioni interpretative che convivono nell'islam, la ricchezza delle posizioni intellettuali e politiche espresse nel suo seno, il gran numero di Stati a maggioranza musulmana, che spesso si qualificano come islamici nella denominazione ufficiale. Dal punto di vista metodologico, ciò impone l'individuazione precisa e contestualizzata delle fonti che vengono selezionate

Data la natura dell'argomento che viene trattato in questo ciclo di lezioni che ha alla sua base la Risoluzione adottata dall'Assemblea Generale dell'Onu il 25 settembre 2015, pare opportuno prendere le mosse dal processo che ha condotto alla sua elaborazione.

L'Agenda 2030 si distingue per il processo estremamente inclusivo che è stato adottato per individuarne i contenuti. Per la prima volta, infatti, nelle consultazioni che hanno 
preceduto la stesura del documento sono stati coinvolti anche attori religiosi ${ }^{2}$.

Va detto per inciso che l'obiettivo più problematico dal punto di vista delle religioni si è rivelato il quinto, relativo all'uguaglianza di genere. Ma esso esula dall'argomento della lezione di oggi.

Vi rientra invece quello relativo alla pace. Il tratto più sorprendente della formulazione di questo obiettivo, e cioè l'assenza di ogni riferimento alla guerra, che pure era presente nei documenti preparatori, non ha nulla a che fare con l'azione degli attori religiosi. Ma l'obiettivo in sé è delicato dal punto di vista delle religioni, il cui ruolo è ambivalente: esse possono infatti esacerbare i conflitti, o contribuire a pacificarli.

Per quanto riguarda l'islam, i due soggetti che possono in un certo senso essere considerati portatori di visioni islamiche e che sono stati coinvolti nella elaborazione del documento e nell'individuazione degli obiettivi, così come nella loro implementazione, sono Islamic Relief e l'OIC (Organizzazione della Cooperazione Islamica).

Islamic Relief è un'organizzazione umanitaria e di sviluppo indipendente che ha come missione quella di aiutare le comunità a fronteggiare e difendersi dai disastri naturali, promuovere lo sviluppo sostenibile, sostenere i bisognosi e combattere le cause della povertà. È particolarmente attiva sui temi della protezione dell'ambiente e del cambiamento climatico. La sua attività è ispirata ai valori islamici di eccellenza, compassione, giustizia sociale e custodia del creato.

Islamic Relief è stata attiva nel processo di definizione dell'Agenda 2030 con incontri, organizzazione di eventi e diffusione di proprie pubblicazioni che sostengono risposte religiosamente ispirate ai temi dello sviluppo. Islamic Relief riconosce nell'Agenda 2030 un documento in linea con le proprie posizioni.

L'OIC è un'organizzazione internazionale che riunisce 57 stati, con delegazione permanente presso le Nazioni Unite. Essa intende rafforzare i rapporti tra i popoli musulmani, rivitalizzare il ruolo dell'islam nel mondo, assicurare 
lo sviluppo sostenibile per i popoli degli stati membri e promuovere i valori islamici di pace, compassione, tolleranza, uguaglianza, giustizia e dignità.

L'OIC non è portatrice di una propria posizione unitaria nell'elaborazione dei documenti internazionali che vengono sottoscritti dagli stati membri. Non sono dunque rinvenibili documenti relativi a questa fase per l'Agenda 2030. L'Organizzazione svolge invece un ruolo importante nella fase della sua implementazione, coordinando le azioni dei vari stati: in particolare, raccoglie i dati provenienti dai vari stati, per monitorare i progressi di ciascuno rispetto agli SDG.

Il suo Comitato permanente per la cooperazione economica e commerciale (COMCEC) e la Islamic Development Bank (IDB) hanno condotto uno studio sui fattori di implementazione dei SDG nei paesi OIC. La IDB uno studio sul contributo delle proprie istituzioni nel raggiungimento dei SDG.

La definizione degli indicatori per misurare l'avanzamento dell'implementazione è stata affidata al SESRIC (Statistical, Economic and Social Research and Training Centre for Islamic Countries), che si è anche occupato di identificare le priorità degli stati membri, al fine della pianificazione delle attività OIC.

Nelle attività programmate, i quattro obiettivi qui in esame sono presenti con frequenza decrescente nel seguente ordine: SDG 10, soprattutto con riguardo ai temi della migrazione e dei profughi, SDG 4, SDG 11 e SDG 16, con riguardo a crimine, conflitti armati e terrorismo.

Emerge costante il riferimento fatto da molti stati membri alle difficoltà finanziarie che si frappongono alla realizzazione degli obiettivi. Ad esse si collegano la limitata disponibilità di tecnologie e le conseguenti difficoltà nella raccolta dei dati necessari a sviluppare politiche efficaci.

A proposito della penuria di risorse finanziare, nei documenti dei più diversi tipi riconducibili a fonti che possono essere connotate come islamiche, ricorre costantemente l'idea che gli obiettivi dello sviluppo sostenibile potrebbero essere finanziati attraverso il sistema finanziario islamico a cui le organizzazioni internazionali dovrebbero riconoscere un maggior ruolo. Non mancano però sottili distinguo. Islamic Relief, per esempio, si riferisce alla «finanza etica islamica», autenticamente ispirata allo spirito e all'etica dell'islam, per 
distinguerla dalla finanza islamica che, come quella convenzionale, ha scelto di puntare alla massimizzazione del profitto.

L'OIC si incarica anche di informare gli stati membri sulla presentazione dei report nazionali volontari da parte degli stati membri sui progressi realizzati rispetto ai $\mathrm{Sdg}$, così da condividere le esperienze e rendere più efficace la cooperazione sui diversi obiettivi. Non tutti gli stati sono ugualmente solerti nella presentazione: il Togo ne ha presentati tre, altri, fino a questo momento, neppure uno. La mancata presentazione potrebbe di per sé indicare la situazione di difficoltà dello stato, ma anche un suo ridotto impegno della realizzazione dell'Agenda.

Sarebbe senz'altro interessante esaminare l'insieme dei report nazionali volontari, per avere un panorama complessivo del posizionamento dei paesi membri dell'OIC rispetto agli obiettivi dello sviluppo sostenibile e degli aspetti che considerano più rilevanti. Ci limitiamo qui a considerare $\mathrm{i}$ report di tre stati, l'Arabia Saudita (2018), il Bahrein (2018) e gli Emirati Arabi Uniti (2018). Pur appartenendo tutti alla medesima area geografica e linguistica, il Golfo Arabo, essi manifestano diversità rilevanti.

Per limitarci alla comparazione delle posizioni dei tre stati rispetto a un obiettivo molto significativo, quello della riduzione delle diseguaglianze, si è colpiti dal fatto che il report dell'Arabia Saudita prende in considerazione soltanto le diseguaglianze di tipo economico. La dimensione culturale e religiosa delle diseguaglianze è invece presa in considerazione dagli altri due report. Quello del Bahrein elenca le raccomandazioni per la riduzione delle diseguaglianze religiose che il paese ha accettato e si propone di realizzare. Gli Emirati Arabi Uniti possono invece dar conto di alcune misure già adottate per la riduzione delle disuguaglianze sia religiose che culturali, tra cui l'istituzione il Ministero per la tolleranza e l'adozione di normative antidiscriminazione.

Se dai documenti che rispecchiano le posizioni di stati e organizzazioni internazionali si sposta l'attenzione alla dottrina, allora ci si trova a confronto con una produzione sterminata. Anche qui, in considerazione della destinazione di questi appunti a una breve lezione, mi limiterò a citare due tipi di critiche che da parte islamica possono essere formulate all'agenda. 
Comunità umane solidali e inclusive
Come accade per tutta l'attività di produzione internazionale di documenti sui diritti dell'uomo in genere, vi è la posizione conservatrice che ne suggerisce il carattere superfluo. Quanto è detto in tali documenti, e nel caso specifico nell'agenda 2030, non aggiunge alcunché a ciò già stato dichiarato più di un millennio fa dalle fonti dell'islam, il Corano e la sunna del profeta ${ }^{3}$.

Altre critiche sono più radicali. Per esse l'Agenda difetta di spiritualità. Le sfere economiche, sociali e ambientali devono essere vivificate rispettivamente dagli ideali islamici di giustizia, fratellanza e dall'idea che l'uomo è il vicario di Dio, e quindi semplice custode della terra, di cui non è padrone. Inoltre l'impostazione dell'Agenda, come di tutti i documenti internazionali, mette al centro il benessere umano, anziché la realizzazione del volere divino ${ }^{4}$.

La prospettiva islamica sugli SDG è estremamente ricca e sfaccettata, e questo intervento, nella sua brevità, intende solo darne una visione impressionistica. La speranza è che possa inserirsi come un tassello in un più vasto mosaico.

3. S. Mukhtar et al., Islamic law and sustainable Development Goals, «Tazkia Islamic Finance and Business Review», XII (2018), n. 1, pp. 81-99.

4. D. Atih Rohaeti et al., A New Approach for Sustainable Development Goals in Islamic Perspective, «Social and Behavioural Sciences», CCXIX (2016), pp. 159-166. 


\section{Il dovere di istruire e di istruirsi nel diritto ebraico}

Bianca Gardella Tedeschi

1. Il diritto allo studio nell'ebraismo e nelle principali carte dei diritti

Lo studio e l'istruzione sono una parte centrale della vita ebraica. Tutti i ragazzi devono poter leggere l'intera parashà, o almeno una porzione, in occasione del proprio Bar Mizwà ${ }^{2}$ Ciò significa che con la pubertà, tutti devono essere in grado di leggere e scrivere. Lobbligo è per la lettura della Torah, che è scritta in ebraico, ma ciò comporta che, comunque, a partire dai 13 anni nessun ragazzo può essere analfabeta. Se a questo si aggiunge, come poi vedremo, che ogni individuo deve dedicare una parte del tempo della vita adulta allo studio, possiamo capire quanto l'istruzione dell'individuo e, quindi, dell'intera comunità sia assolutamente essenziale nel determinare lo stile di vita nell'ebraismo. Se il popolo ebraico è chiamato il popolo del libro è dovuto a molti fattori, tra cui le fonti del diritto ebraico e le particolari tecniche interpretative, ma tra questi

1. Maggiorità religiosa, con cui si entra nell'età adulta. Per i ragazzi è fissata a 13 anni, per le ragazze a 12 . 
II dovere di istruire e di istruirsi nel diritto ebraico è inclusa la capacità di lettura e analisi di un testo scritto che ciascuno acquisisce.

Nel mondo occidentale, il diritto ad una istruzione si inserisce all'interno dei diritti fondamentali sociali. L'art. 34 della Costituzione italiana è dedicato al diritto all'istruzione, prevedendo sia l'istruzione obbligatoria che il proseguimento degli studi superiori per gli alunni più meritevoli ${ }^{2}$. La convenzione Europea dei diritti dell'uomo lo contempla all'art. 2, protocollo 1. Da ultimo, la Carta dei Diritti Fondamentali dell'Unione Europea (o carta di Nizza) del 7 dicembre 2000, introduce, con l'art. 14, il diritto all'istruzione e l'accesso alla formazione professionale continua attraverso la garanzia di un'istruzione obbligatoria.

Lo stesso diritto è riconosciuto anche a livello internazionale. È previsto infatti dall'art. 26 della Convenzione internazionale dei diritti umani del 1948, che ne sancisce la gratuità e l'obbligatorietà. L'art. 28 e 29 della Convenzione internazionale sui diritti dell'infanzia e dell'adolescenza (1989) prevedono obbligatorietà, gratuità e finalità dell'istruzione. Lart.13 del Patto Internazionale sui diritti economici, sociali e culturali (1966) prevede il diritto all'istruzione come base per la convivenza sociale e la partecipazione degli individui alla vita politica e alla costruzione della pace.

Il diritto all'istruzione è oggi promosso dalle Nazioni Unite nell'Agenda 2030 per lo sviluppo sostenibile come uno degli obiettivi da raggiungere universalmente entro il 2030. Si tratta dell'obiettivo n. 4 dell'Agenda, denominato "Istruzione di qualità". "Un'istruzione di qualità è la base per migliorare la vita delle persone e raggiungere lo sviluppo sostenibile": questa è la frase con cui si apre la descrizione dell'obiettivo numero 4 che lega l'istruzione alla qualità di vita delle persone. Gli obiettivi da raggiungere sono elencati successivamente, dopo aver illustrato alcuni dati sul livello di istruzione nel mondo. Tra gli obiettivi, garantire

2. Cfr. R. Calvano, Scuola e Costituzione, tra autonomie e mercato, Roma 2019; F. Angelini, M. Benvenuti (a cura di), Le dimensioni costituzionali dell'istruzione, Napoli 2014; P. Calamandrei, Contro il privilegio dell'istruzione, «Il Ponte», 1, 1946, pp. 4-5, e rist. in Id., Per la Scuola, Palermo 2008, p. 112; V. Crisafulli, La Scuola nella Costituzione, «Riv. trim. dir. pubbl.», 1956, pp. 55 sgg. Da ultimo, L. Conte, La scuola si è fermata. L'impatto del Covid-19 sui diritti all'istruzione, all'educazione e all'insegnamento, in A. Poggi, F. Angelini, L. Conte, La scuola nella democrazia. La democrazia nella scuola, Editoriale Scientifica, Napoli 2020. 
ai ragazzi e alle ragazze accesso alla scuola primaria e secondaria, garantire una formazione tecnica e professionale adeguata ai giovani adulti, aumentare il numero delle borse di studio per permettere a chi non ha i mezzi di istruirsi e formarsi, abolire le disparità di genere nell'apprendimento.

Il diritto ebraico ha da sempre espresso una particolare attenzione nei confronti dell'istruzione e della formazione, sia religiosa che professionale. In questo senso, come vedremo, il diritto ebraico può essere considerato tra i sistemi giuridici che si pongono già nel segno indicato dall'agenda Onu 2030.

\section{Diritto o dovere di istruzione?}

Nella tradizione giuridica occidentale, l'istruzione è configurata come un diritto fondamentale. L'espressione "diritti umani”, al cui interno risiede il diritto a un'istruzione, è invece assente dalle fonti ebraiche ${ }^{3}$. Il diritto ebraico si esprime nel linguaggio del dovere e delle obbligazioni. Anche l'istruzione rientra in questa visione: l'ebraismo declina l'istruzione come un dovere di istruire e un dovere di istruirsi. Robert Cover esplora il concetto di obbligazione come pietra angolare nella costruzione dell'ordine sociale ebraico. Per spiegare la peculiarità di essere titolari di obblighi, piuttosto che di diritti, Cover prende ad esempio proprio l'istruzione e spiega come sia importante l'accento sul dovere in quanto il dovere è migliorativo del benessere comune 4 . Infatti, il "diritto all'istruzione" preso da solo esplicita un bisogno di istruzione, senza però precisare chi sia il soggetto obbligato a fornirla. Il "diritto" non prevede un destinatario che prenda in mano il soddisfacimento di

3. M.R. Hayoun, Judaïsme et droits de l'homme, in M. Di Marco, S. Ferrari, Ebraismo e cristianesimo nell'età dei diritti umani, Milano 2018, p. 23; B. Gardella Tedeschi,Ebraismo e diritti umani: una prospettiva dal diritto positivo, in M. Di Marco, S. Ferrari, Ebraismo e cristianesimo cit., p. 85; H. Dagan, Sh. Lifshitz, Y. Z. Stern (curr.), Religion and the Discourse of Human Rights, The Israel Democracy Institute, Jerusalem 2014; D. Wermuth, Human Rights in Jewish Law: Contemporary Juristic and Rabbinic Conceptions, 32 «U. Pa. J. Int'l L.» 1101 (2011 A. Maoz, Can Judaism Serve a Source of Human Rights, «Tel Aviv University Law School Faculty Papers», 2005, n. 7; M. Konvitz (cur.), Judaism and Human Rights, 2a ed., New Brunswick - NJ, London 2001; S. Daniel Breslauer, Judaism and Human Right. A Bibliographical Survey, Westport, Ct 1993; M. Broyde, J. Witte (curr.), Human Rights in Judaism, Northvale. NJ, Jerusalem 1984).

4. R. M. Cover, Obligations: A Jewish Jurisprudence of the Social Order, 6 «J.L. \& Religion», 65 (1987). 
Il dovere di istruire e di istruirsi nel diritto ebraico un diritto e rimane "solitario". Nel diritto ebraico, invece, è previsto il dovere di fornire una istruzione, che grava poi su differenti soggetti: i genitori, altri soggetti oppure la comunità. Ciò significa che il destinatario finale dell'obbligazione di istruire sa sempre a chi rivolgersi per ottenere ciò che è posto come dovere dal Signore stesso. Il primo destinatario dell'obbligo è il padre e questo mette la famiglia al centro dell'attività di apprendimento dei fanciulli. In questa accezione, la famiglia non è solo il luogo degli affetti o di una generica "educazione" alle "buone maniere" ma è anche il luogo di trasmissione della conoscenza acquisita dai singoli che la passano alle generazioni successive. La famiglia non è, però, considerata sufficiente nella trasmissione del sapere e a questa si affianca un maestro.

\section{I sistemi scolastici nella storia del popolo ebraico}

Proprio per adempiere al dovere di istruire i ragazzi, è sempre presente all'interno delle comunità ebraiche, in ogni epoca e ad ogni latitudine, un sistema scolastico organizzato $^{5}$. Ci sono delle differenze, anche notevoli, a seconda della zona geografica, con maggiori differenze, in epoca medioevale tra la Spagna e l'Europa del Nord, e del periodo storico. Ma la scuola in cui i ragazzi imparano a leggere e scrivere, a cominciare dall'ebraico, è sempre presente ${ }^{6}$. Già all'epoca del diritto biblico, accanto al padre c'era un maestro per insegnare ai ragazzi. Le fonti storiche ci mostrano come già nel periodo del secondo tempio fosse presente una rete di scuole, istituite con un'ordinanza secondo cui i maestri dovevano essere presenti in ogni distretto, e non solo più a Gerusalemme. L'età in cui si cominciava a frequentare la scuola fu fissata con la medesima ordinanza

5. Linsegnamento della Torà è obbligatorio per i ragazzi e facoltativo per le ragazze. Per la cultura secolare, ci sono stati diverse posizioni nel corso della storia, che riflettevano le diverse tradizioni nelle diverse regioni regioni geografiche ma è corretto affermare che anche alle ragazze era impartita una istruzione di base. Cfr. la voce "Education" della Encyclopedia Judaica (Jerusalem 1971, vol. 6) che mette in evidenza, quando rilevante, la qualità dell'istruzoine impartita alle ragazze. Sull'insegnamento religioso alle donne, cfr. B. Gardella Tedeschi, La condizione giuridica delle donne nel diritto ebraico: un'introduzione, in I. Zuanazzi, M.C. Ruscazio, Le relazioni familiari nel diritto interculturale, Tricase (Le) 2018, p. 166.

6. Le notizie storiche sono tratte dalle differenti voci dell'Encyclopedia Judaica (Jerusalem 1971) raggruppate all'interno della voce principale "education" (vol. 6). Nei luoghi appropriati, è indicata la corrispondente sottovoce. 
ai sei o sette anni. Si era ritenuto, infatti, che dopo questa età i ragazzi non fossero più disponibili a imparare e quindi abbandonassero gli studi ${ }^{7}$.

Un sistema di scuole destinato ai più giovani è poi sempre stato presente nella vita delle Comunità ebraiche della Diaspora, con caratteristiche diverse, a seconda del luogo e della cultura all'interno della quale la Comunità ebraica si trovava a vivere. Ma l'esistenza di una scuola, anche nelle situazioni più estreme, è stata una caratteristica imprescindibile della vita ebraica.

Ibn Aknin, un maestro vissuto in Nord Africa a cavallo del XII e del XIII secolo, esprime idee e opinioni tipiche dell'ebraismo sefardita del suo tempo. A lui si deve il primo programma del curriculum scolastico ${ }^{8}$ : lo studio comprende la Torah, la Mishnà e il Talmud. A questi, si aggiungono materie secolari: grammatica, poesia, logica, retorica, aritmetica, geometria, astronomia, musica, scienze naturali e metafisica. Ibn Aknin delinea anche le caratteristiche di un bravo maestro. Un bravo maestro deve sapere bene ciò che insegna, deve mettere in pratica ciò che insegna, essere paziente con gli studenti e soppesare le loro capacità di comprensione. Inoltre, deve mettere particolare enfasi sugli aspetti etici del suo insegnamento. Oltre alle caratteristiche del maestro, Ibn Aknin ci illlustra come deve comportarsi l'allievo: deve essere pulito ed educato, deve essere attento e seguire la disciplina, non deve essere pigro e deve studiare per amore della conoscenza e non per acquisire ricchezza, o comunque per altri scopi diversi dalla sola conoscenza. Uno studente maturo deve cercare di frequentare le scuole migliori e, se possibile, imparare da bravi maestri e non esclusivamente dai libri. Questo curriculum, ci riportano le fonti, era seguito in molte comunità della Spagna. Un altro maestro, Judah ibn Abbas, suggerisce una scansione temporale più precisa, a partire dai 4-5 anni per culminare ai diciotto anni con lo studio dei soggetti secolari: medicina, matematica, astronomia, logica e scienze naturali.

Le materie secolari non facevano invece parte del curriculum dello studente che viveva nella Francia del Nord

7. Ł̀ questo il contenuto dell'ordinanza di Rav ben Gamla, TB Bava Batra 21a, 4. Cfr. Nathan Drazin, History of Jewish Education from 515 B C E to 220 C E, New York, NY 2015.

8. Encyclopedia Judaica, voce "Education" cit., the western Mediterrenean Lands, c. 405. 
Il dovere di istruire e di istruirsi nel diritto ebraico o in Germania. In questa realtà, il curriculum consisteva esclusivamente nello studio della Torah, scritta e orale, e nell'apprendimento delle regole da osservare ${ }^{9}$. Tutti erano quindi alfabetizzati: alcuni studiosi ritengono che nella Francia dell'anno Mille non ci fosse un ebreo che non fosse in grado di leggere e scrivere, in ebraico. L'inizio della istruzione scolastica, il primo giorno di scuola, era un evento speciale nella vita di una persona. Si andava a scuola lavati e ben vestiti, con una lavagna e buona abbondante merenda: pane, mele, uova sode e altra frutta. Ha inoltre una lavagna. Un uomo saggio della Comunità lo accompagna il primo giorno di scuola. Il bambino ha 5 anni. Rispetto alla Spagna, si ha un'importante differenza: la filosofia, o altre discipline secolari, non attiravano lo studioso di tradizione ashkenazita.

Data la grande importanza data dalla Halachà all'istruzione, e visti gli alti costi che questa comporta, molto spesso è intervenuta la comunità per garantire che tutti potessero avere una istruzione. Già nell'epoca dei Gheonim ${ }^{10}$, i maestri erano assunti dalle comunità e considerati funzionari della comunità $^{11}$. In Spagna, fino alla cacciata del 1492, erano istituite tasse comunitarie e organizzate raccolte di fondi e donazioni per sostenere le scuole ${ }^{12}$. La scuola era per tutti, indipendentemente dal reddito. Meir Abulafia, che operò in Spagna nel xiı secolo, in un responso, afferma che le comunità devono assumere i maestri per gli studenti ed è un dovere dell'intera comunità, e non solo dei genitori dei bambini, pagare gli insegnanti. Sempre in Spagna, a Valladolid, erano soggetti a tassazione alcuni servizi della Comunità, tra cui la carne, il vino, matrimoni, funerali o circoncisione, ed era stabilito che questi proventi andassero esclusivamente a coprire le spese per la scuola. Inoltre, ogni comunità che avesse almeno 15 gruppi familiari doveva avere almeno un maestro e laddove le contribuzioni

9. Encyclopedia Judaica, voce "Education" cit., Northern France and Germany, c. 408. 10. I Gheonim, o eccellenze, erano i capi delle accademie talmudiche di Babilonia dalla fine del v secolo fino all'xi secolo in occidente e al xin in Oriente (A.M. Rabello, Introduzione al diritto ebraico, Milano 2002, p. 59.

11. Encyclopedia Judaica, voce "Education" cit., Babylonian, Pre-Gaonic and Gaonic Periods, c. 404.

12. Encyclopedia Judaica, voce "Education" cit., Community Responsibility, c. 412. 

maestro, la differenza era coperta dalla comunità. Anche nel Nord Europa esisteva una responsabilità piuttosto ben normata dell'istruzione a carico delle comunità. Rabbenu Tam, figlio del grande commentatore Rashi, nelle sue ordinanze riferisce che le comunità si facevano carico delle spese del maestro, e questa era una pratica consolidata. Rabbenu Tam statuì che nel caso in cui si fosse all'interno della comunità una carenza di fondi, le somme destinate ad altre attività dovessero essere riallocate per coprire il fabbisogno di istruzione. Le comunità renane accettarono queste ordinanze nel 1220. La regolamentazione riguardava anche altri aspetti della vita scolastica, quali il numero degli allievi per ogni classe, supervisione dei maestri, e in alcune scuole più numerose era presente del personale ausiliario non destinato all'insegnamento.

La pratica di avere scuole per ogni comunità è continuata nei secoli successivi, anzi fu poi resa più semplice dalla diffusione della stampa che rese accessibili, anche per il prezzo, i libri ${ }^{13}$. Nonostante le persecuzioni e le espulsioni, ricorrenti in tutti i periodi storici, l'istruzione rimase una priorità sia per le famiglie che per le comunità ebraiche. Il curriculum cambiò molto a partire dal XviI secolo, dal momento che ci fu una graduale apertura delle comunità ebraiche al mondo esterno. Molti appartenenti alle comunità ebraiche, soprattutto coloro che avevano numerosi scambi con la popolazione non ebraica, persero interesse nell'apprendimento dell'ebraico e della tradizione ${ }^{14}$. Molti trovavano che fosse inutile. Si sentiva invece il bisogno di conoscere le lingue straniere e avere l'educazione secolare per poter esercitare la propria professione. Il passaggio dal mondo tradizionale al mondo industriale e la voglia di molti di passare dal mondo "tradizionale" a quello "moderno" è catturato con particolare efficacia da Singer nel romanzo "I fratelli Ashkenazi" "15, che segue la vita di due fratelli, figli di un padre studioso e colto nell'ebraismo, che diventano

13. Encyclopedia Judaica, voce "Education" cit., Jewish education, $16^{\text {th }}-18^{\text {th }}$ century, c. 413 . 14. Encyclopedia Judaica, voce "Education" cit., Jewish education, 16th-18th century, Europe - North and East, c. 413.

15. I.J. Singer, I fratelli Ashkenazi, Torino 2011, traduzione di B. Fonzi, Introduzione di C. Magris. 
II dovere di istruire e di istruirsi nel diritto ebraico

invece importanti industriali. Devono imparare il tedesco e il russo, lingue che non conoscevano, e di cui porteranno sempre un piccolo accento che rivela le loro origini, e si trasformano, nell'arco di pochi anni, in ebrei nuovi, abbandonando il nome ebraico a favore di un nome tedesco e cambiando il modo di vestire. Il passaggio dal gabbano tradizionale agli abiti alla moda è emblematico di questa trasformazione interiore.

Nella seconda metà del xviı secolo, la condizione degli Ebrei tedeschi e dell'Impero austro-ungarico cambiò, poiché ci fu una parziale emancipazione e una rimozione di alcune restrizioni che li tenevano in disparte rispetto al resto della popolazione. Il maggiore contatto con le popolazioni locali cambiò la domanda di istruzione da parti della popolazione ebraica che portò alcuni studiosi a ripensare il curriculum scolastico. Il maggiore esponente fu Naphtali Hirtz Wessely, fautore e sostenitore della modernizzazione delle scuole ebraiche, attraverso l'introduzione, accanto al curriculum tradizionale, delle materie del mondo moderno, quali le lingue, a partire da quella parlata nel proprio territorio, aritmetica, geografia, storia, buona educazione. La riforma non fu accolta con favore dai più tradizionalisti ma fece presa sulla maggioranza di coloro che volevano liberarsi della atmosfera del ghetto. Anche i governi sostennero economicamente questa modernizzazione, ritenendo che sarebbe stato un miglioramento nell'impegno civile dei cittadini ebrei.

L'Italia si è trovata in una situazione diversa da quella dell'Europa del Nord e dell'Est ${ }^{16}$. Dopo l'espulsione degli Ebrei dalla Spagna nel 1492, l'Italia costituiva la più larga comunità ebraica del Mediterraneo. In Italia, rimasero alcune caratteristiche tipiche dell'istruzione impartita in Spagna: il programma scolastico comprendeva infatti sia la lingua locale, che l'aritmetica e la composizione. Gli insegnanti delle materie secolari erano spesso cristiani. Scopo dell'istruzione era di formare delle persone che potessero essere allo stesso tempo buoni Ebrei e persone a proprio agio nella società in cui vivevano. Una caratteristica parti-

16. Encyclopedia Judaica, voce "Education" cit., Jewish education, 16th-18th century, Itay, c. 417 . 
colare dell'Italia fu la presenza della "maestra" e non solo del "maestro" per i bambini più piccoli.

In epoca più vicina a noi, con la definitiva emancipazione degli Ebrei, i diversi sistemi scolastici organizzati dalle comunità subirono profondi cambiamenti. In epoca contemporanea, declina l'interesse per gli studi religiosi, tanto che spesso è difficile trovare non solo gli allievi ma gli stessi maestri. Allo stesso tempo, gli stati nazionali organizzano un sistema di scuola pubblica obbligatoria che toglie alle comunità ebraiche la piena disponibilità del tempo dei propri ragazzi. Il dovere comunque di impartire un'educazione ai propri figli continua certamente ad essere molto sentito nelle famiglie ebraiche di ogni parte del mondo.

\section{Le fonti}

Nel diritto e nella tradizione ebraica, come abbiamo detto, un posto particolare è occupato dal dovere di fornire un'adeguata istruzione ai bambini, così come $\mathrm{i}$ genitori hanno l'obbligo provvedere al cibo e ai vestiti per i propri figli. L'obbligo di trasmettere la parola del Signore, è contenuta in Deuteronomio 29,11: «le insegnerete ai vostri figli, parlandone con loro stando in casa, quando cammini per via, quando ti coricherai e quando ti alzerai». I giovani sono anche invitati a domandare ai padri e ai più anziani per imparare da loro: "Ricorda i tempi antichi, cercate di comprendere gli anni dei secoli trascorsi, interroga tuo padre e ti racconterà, i tuoi vecchi e te lo diranno» (Deut. 32,7 ${ }^{17}$.

Il dovere è in primo luogo del padre. Se questi non può adempiere al suo dovere, sono previsti dei sostituti nell'obbligo ${ }^{18}$. Quale ultima risorsa, rimane la Comunità ebraica di appartenenza che è tenuta a dare un'istruzione ai bambini i cui genitori non possono adempiere, per motivi diversi, o perché deceduti, o perché non possono permettersi di sostenere i relativi costi. Il dovere di fornire una adeguata istruzione è quindi non solo della famiglia ma è un dovere che spetta all'intera società. Il

17. Pentateuco e Haftaroth, con Traduzione italiana e note, Torino, 1960. Deuteronomio a cura di Rav Elio Toaff.

18. Su questo punto, non c'è concordia tra i commentatori: si tratta di un dovere del Tribunale, che può agire direttamente oppure attraverso la nomina di un tutore ad hoc. Cfr. M. Broyde, Why Educate? A Jewish Law Perspective cit., 183, n. 18. 
Il dovere di istruire e di istruirsi nel diritto ebraico dovere investe tutti i campi del sapere. Non si tratta solo di fornire un'educazione religiosa, ma anche di fornire un'educazione morale e, seppure con diversità di opinioni in proposito, una istruzione secolare. Il dovere è un dovere che unisce indissolubilmente l'area del diritto con quello dell'imperativo etico ${ }^{19}$.

Pur essendo lo studio e la formazione così centrale per conservare e tramandare l'ebraismo alle generazioni successive, non abbiamo all'interno del Talmud una sezione dedicata all'istruzione, o comunque una parte in cui questo argomento sia affrontato sistematicamente con una trattazione halachica esaustiva ${ }^{20}$. Ciononostante, il Talmud, in diversi passi, sottolinea l'importanza dei genitori e della Comunità di dare una adeguata istruzione ai giovani. In Kiddushin 29b, si trova il riferimento all'obbligo del padre di insegnare ai propri figli. In Kiddushin 30a, l'obbligo viene ripetuto. Sempre in Kiddushin 30a, ci sono alcune precisazioni sul modo di studiare: non bisogna solo ripetere ciò che si studia. Il processo è diviso in tre fasi: bisogna studiare, rivedere e poi studiare nuovamente. In Bava Batra 21a,4, si dà conto che Yeohusha ben Gramla ha ordinato la presenza di un maestro in ogni distretto, e non solo nella capitale, Gerusalemme, dove i ragazzi potevano cominciare a frequentare le lezioni a partire dai sei o sette anni. In Bava Batra 21a,5, si affrontano alcuni aspetti dell'insegnamento, tra cui l'effetto positivo della collaborazione tra studenti, in cui chi sa di più incoraggia chi sa di meno. In Ketubot $50 a, 10$, si affronta la questione se sia saggio o meno far iniziare la scuola prima dei sei anni. Le opinioni sono ovviamente divise tra chi sostiene, a partire da un detto di Rav Ketina, che andare a scuola prima dei sei anni indebolisce i ragazzi e chi invece sostiene che li fa avanzare più velocemente. Un ragazzo già debole, però, non deve iniziare troppo presto la scuola.

Una fonte più ordinata per questa materia è lo Schulchan Aruch, la raccolta di regole ebraiche redatta da Rav Joseph Caro nel xvi secolo, che dedica uno spazio ampio al dovere di istruzione dei propri figli.

19. M. Broyde, Why Educate? A Jewish Law Perspective, 44 «Journal of Catholic Legal Studies», 179 (2016).

20. Encyclopedia Judaica, voce "Education", Jerusalem 1971, vol. 6, c. 398. 
Qui, si afferma che ogni persona deve insegnare a suo figlio la Halachà ${ }^{21}$. Proprio il fatto che il dovere sia importo a ciascuna persona, fa vedere l'esistenza comunque del dovere di insegnamento anche quando il dovere, inteso in senso letterale, non è applicabile: ciò significa che, chi è in grado, deve insegnare all'interno della propria comunità o provvedere all'educazione di altri ${ }^{22}$. Il "programma" scolastico è delineato con attenzione: quando il figlio inizia a parlare, gli si può insegnare che Mosé ha ricevuto sul Monte Sinai la Torah, e insieme gli si insegna il principio dell'unità del Signore. Dopo gli si insegna qualcosa in più, fino a che a sette anni dovrà essere condotto un maestro. Il maestro, se l'uso locale è di insegnare dietro compenso, non potrà lasciare il bambino fino a che questi non abbia completato il programma, che consiste nel saper leggere l'intera Torà. Non è invece tenuto a insegnare la Mishnà o la Gemarà, se ci sono circostanze che lo impediscono, ad esempio la mancanza di tempo. Se invece è possibile, deve insegnare anche queste.

Sempre lo Schulchan Aruch, prevede che ogni Comunità costituisca un sistema scolastico. In ogni Comunità deve infatti essere presente una scuola elementare, per l'importanza dello studio dei bambini per l'intera umanità. Anche le regole di condotta interne della scuola sono contenute all'interno dello Schulchan Aruch: la classe deve essere composta al massimo di 25 studenti. Se il numero degli allievi è compreso tra i 25 e i 40, allora deve essere previsto un aiuto-maestro. $\mathrm{Al}$ di sopra dei 40, è necessario dividere la classe e avere un secondo maestro.

Sempre lo Schulchan Aruch considera un miglioramento per la popolazione tutta la fondazione di più scuole ed è questo uno dei pochi casi in cui si soprassiede ad alcune regole sulla concorrenza ${ }^{23}$. Così, un maestro può aprire una nuova scuola vicino ad una già esistente, pur se questo comporta una perdita di clienti per la scuola già esistente, non è possibile impedire questo comportamento. Sempre nella stessa direzione, lo Schulchan Aruch ci dice che se un

21. Schulchan Aruch, Yoreh De'ah, 245:1.

22. M. Broyde, Why Educate cit., p. 181, n. 10.

23. Ivi, p. 183. 
Il dovere di istruire e di istruirsi nel diritto ebraico proprietario vuole insediare una nuova scuola, ad esempio nel cortile di casa sua, non è possibile impedirglielo, adducendo ragioni urbanistiche.

\section{Il contenuto del dovere di impartire una istruzione ai propri figli}

Quale direzione deve prendere la preparazione fornita ai fanciulli? L'istruzione che deve essere impartita è plurima: si ha il dovere di trasmettere ai giovani un'adeguata preparazione religiosa, ma non solo. Il dovere comprende anche il dovere di impartire gli insegnamenti che possano poi permettere di poter lavorar e mantenersi, quindi quegli insegnamenti secolari che sono necessari per poter aver una professione e un lavoro. Il termine in ebraico che si usa per parlare di istruzione è "hinukh" che significa "inizio" o "preparazione", a mettere in evidenza come l'istruzione non possa essere disgiunta dal suo scopo intrinseco che è quello di preparare, appunto, per la vita adulta.

Lo scopo della preparazione dei fanciulli è, quindi, fornire loro quegli strumenti che saranno poi necessari nella loro vita adulta. Bisognerà quindi renderli familiari con la Torah e con le regole da osservare. Per il diritto ebraico, chi non ha raggiunto la maggiore età religiosa, 13 anni per i ragazzi e 12 per le ragazze, non è tenuto al dovere di osservanza delle mizwot, cioè i doveri contenuti nella Torà ${ }^{24}$. Ma i bambini vengono già abituati fin da piccoli a conoscere le regole della vita ebraica ed osservarle, quale preparazione per la vita da adulti.

L'educazione dei fanciulli da parte dei genitori non consiste esclusivamente nella trasmissione delle regole e delle tecnicalità della Halachà. Il dovere principale è quello di educare il proprio figlio nella sul sentiero, stretto e accidentato, della vita e di fornirgli l'indirizzo morale che gli permetta di seguirlo. Broyde riporta il responso della Corte d'Appello rabbinica di Israele, in cui Rav Joseph Kapach puntualizza come ormai gli aspetti più "tecnici" della formazione ebraica siano affidati dai genitori a enti e scuole di formazione. Secondo Rav Kapach, il dovere principale dei 
25. 9 Piskei Din Rabbaniyim 251, 259 (Isr. Rabb. Ct. 1974) citato da M. Broyde, Why Educate? cit., p. 186.

26. TB Kiddushin 29a.

27. Rav Shelomo ben Izchak (Troyes, 1040-1105), commentator classic della Bibbia e del Talmud. Cfr. A.M. Rabello, Introduzione cit., p. $62 \mathrm{sgg}$. 
II dovere di istruire e di istruirsi nel diritto ebraico

\section{Anche gli adulti devono continuare a imparare}

Per la Halachà, cioè l'insieme del diritto ebraico, il dovere di impartire alle persone una istruzione non è limitato ai giovani. Per lo Schulchan Aruch, tutti, qualunque sia la loro condizione di salute, economica, di età, siano sposati o non sposati, devono dedicare tempo allo studio ${ }^{28}$. Gli adulti devono dedicare tempo allo studio ed hanno il diritto di ricevere questa formazione. Il detto di Rabban Gamaliel secondo cui ciascuno deve trovare un maestro ${ }^{29}$, è stato accolto nella tradizione ebraica come diretto a ciascun individuo, indipendentemente dall'età. In definitiva, finito il dovere imposto ai genitori, il dovere di istruirsi ricade direttamente sulla singola persona, ormai adulta ${ }^{30}$. Il dovere menzionato nello Schulchan Aruch è per lo studio dell'ebraismo, delle fonti, della Torah e della Halachà. Va però notato quanto sia importante per questo testo centrale per l'ebraismo che siano dedicati tempo e risorse per l'istruzione degli adulti. Il dovere di fornire una istruzione, o come una continuing education agli adulti, non è normalmente previsto dalle fonti del diritto internazionale, o anche del diritto interno in molti paesi. Il diritto ad ottenere una istruzione è visto soprattutto come un diritto dei fanciulli, non come un diritto degli adulti. Questi accedono ad una formazione dedicata in determinate situazione, di cui la disoccupazione, o la sua minaccia, è un fattore importante. Lo Stato non ritiene sia tra i suoi doveri principali quello di continuare a fornire anche agli adulti una istruzione.

\section{Alcuni principi in tema di istruzione}

Nelle fonti ebraiche, sono espresse molte riflessioni sui principi educativi che stanno alla base di una ottimale trasmissione del sapere ${ }^{31}$. Con "ho imparato da tutti i miei maestri" (Avot 4,1), si vuole porre l'enfasi sul fatto che si può imparare da tutti e che non bisogna, quindi, perdere

28. V. supra, par. 3.

29. Avot 1,16 .

30. M. Broyde, Why educate cit., che fa del dovere imposto a tutti di continuare a studiare durante l'intero arco della vita il tratto caratteristico dell'ebraismo, rispetto alla tradizione giuridica occidentale che considera l'istruzione essenzialmente un diritto dei fanciulli. 31. Urkin, Jacob et al., Nurturing a Society of Learners: Suggestions from Traditional Jewish Pedagogy for Medical Education, «Rambam Maimonides medical journal», vol. 8, 3 ,2017. 
alcuna possibilità di imparare. Con l'espressione "siedi nella polvere ai piedi dei saggi e bevi avidamente le loro parole" si incita l'allievo ad apprendere quanto più gli è possibile dai maestri. La tecnica dell'apprendimento in piccoli gruppi è presente quando si dice ad uno studente di trovarsi un maestro e un compagno di studi (Avot 1,6). Il maestro deve essere flessibile e comprendere i propri studenti, perché "un maestro rigido non può insegnare" (Avot 2,5). L'insegnamento deve essere misurato sui discenti, come è scritto "istruisci un ragazzo secondo le sue abilità" (Proverbi 6,6). La frase con cui si afferma che lo studio di un ragazzo richiede molta fatica, (TB Ta'anit, 7a) sta a indicare che un buon programma facilita l'apprendimento, non sovraccarica i ragazzi. Quindi, un maestro deve osservare bene come gli studenti sonno in grado di seguire ed eventualmente apportare dei cambiamenti al programma. I maestri imparano non solo dalla preparazione individuale, ma dal confronto con i colleghi e soprattutto dal confronto con gli studenti (TB, Ta'anit 7a). Inoltre, bisogna ritagliare sempre un po' del proprio tempo per lo studio perché quando se si pensa di studiare quando ci sarà tempo, non si troverà mai il tempo (Avot 2.4); e non si deve rimandare, perché se si rimanda di un giorno si rimanda anche di due (TG, Berachot 14d): lo studio deve essere una priorità assoluta. 
III. Vita umana, lavoro e sviluppo tecnologico (obiettivi 3, 8 e 9 Agenda Onu 2030)

aA 



\section{III.1. Gli obiettivi dello sviluppo sostenibile}

aA 

L'articolo si concentrerà in particolare sugli obiettivi globali per lo sviluppo sostenibile numero 3, 8 e 9 dell'agenda 2030 delle Nazioni Unite che, come precisa l'introduzione, si reggono all'interno di un progetto volto a raggiungere lo sviluppo sostenibile nelle sue tre dimensioni economica, sociale e ambientale "in maniera equilibrata e interconnessa". Nella visione proposta la protezione del pianeta dalla degradazione ambientale si coniuga con la tutela del diritto di ciascuno a vivere una vita soddisfacente, ove il progresso sia concepito armonicamente con la natura, entro società "pacifiche, giuste, inclusive e libere dalla paura". Il linguaggio adottato è quello della grande tradizione delle dichiarazioni dei diritti umani e del diritto allo sviluppo che hanno preceduto questo testo, ma l'ampiezza e la profondità della visione e dell'interconnessione tra gli obiettivi indicano uno sguardo nuovo e più ambizioso.

L'analisi delle grandi sfide è il punto di partenza che orienta le azioni contro la povertà, la disparità di genere, le minacce alla salute, all'occupazione, l'esaurimento delle risorse naturali e la stessa sopravvivenza dell'equilibrio ecobiologico del pianeta. 
Segue nel testo l'analisi dei risultati raggiunti con i precedenti obiettivi di sviluppo del millennio e delle criticità ancora presenti, sul piano della povertà, del supporto alle persone deboli, della diffusione dell'educazione e la promozione della salute psichica e fisica e dello sviluppo economico (disparità di reddito, sfruttamento del lavoro e dei flussi migratori, degrado ambientale).

La logica che presiede alle azioni è ispirata alla sussidiarietà, riconoscendo la responsabilità di ogni paese per il proprio sviluppo e individuando al tempo stesso la centralità delle istituzioni, finanziarie e non, internazionali e dei parlamenti nazionali.

Lagenda si presenta, nella sua ampiezza antropologica e per la propria visione profetica, quindi, come un vero e proprio "statuto per le persone e il pianeta del xxi secolo".

Lanalisi del singolo obiettivo non deve dimenticare che obiettivi e traguardi sono "interconnessi e indivisibili", riconoscendo al tempo stesso la mancanza di dati da cui si muove - elemento centrale per il tema dell'intelligenza artificiale cui dedicheremo qualche osservazione - come riferimento per i diversi traguardi posti.

Nello specifico gli obiettivi presi in conto in queste note critiche sono l'obiettivo 3 (Assicurare la salute e il benessere per tutti e per tutte le età), l'obiettivo 8 (incentivare una crescita economica duratura, inclusiva e sostenibile un 'occupazione piena e produttiva e un lavoro dignitoso per tutti) e infine il 9 (Costruire un'infrastruttura resiliente e promuovere l'innovazione ed una industrializzazione equa, responsabile e sostenibile).

Nell'agenda gli obiettivi non sono privi di indicatori precisi che li specificano, per l'obiettivo 3 indicando parametri precisi per la riduzione del tasso di maternità globale e l'individuazione dei pericoli da combattere: epidemie, abuso di sostanze stupefacenti, conseguendo "una copertura sanitaria universale", assicurando l'assistenza sanitaria e riproduttiva, l'accesso ai vaccini aumentando i fondi destinati alla sanità e alla formazione del personale sanitario e sviluppando un'azione preventiva di segnalazione e di contrasto dei rischi. Tema su cui non è possibile intervenire in questa sede, oggetto anche del rapporto 2020 dell'ASvIS, che discute l'influenza enorme della pandemia sul programma di attuazione dell'agenda. 
L'obiettivo 8 è volto all'incentivazione della crescita economica, da intendere come 'duratura, inclusiva e sostenibile' e, contestualmente, alla promozione di un'occupazione produttiva, legata a un lavoro dignitoso per tutti e che indica una serie di obbiettivi precisi da conseguire. Essi sono il sostegno della crescita economica pro capite ed annua, nei paesi in via di sviluppo, e una crescita del $7 \%$ del PIL nei paesi in via di sviluppo.

Tale obiettivo dovrebbe essere raggiunto mediante una maggiore produttività ottenuta attraverso il progresso tecnologico e l'innovazione, promuovendo imprenditoria e creatività, come necessariamente legate all'implementazione di un lavoro dignitoso e inclusivo verso le donne e i disabili e riducendo ineguaglianze di retribuzione e disoccupazione, specie quella giovanile: fino a giungere a una lotta senza quartiere verso lo sfruttamento e le forme di schiavitù minorile (entro il 2025, elemento temporale di prossimità che ne individua la particolare rilevanza).

Lobiettivo 9 si concentra sulla costruzione di infrastrutture resilienti, indirizzando l'innovazione verso una industrializzazione 'equa, responsabile e sostenibile', da intendere come supporto allo sviluppo economico. Legati a questo obiettivo sono, secondo l'agenda, a un tempo l'aumento delle quote di occupazione dell'industria (specie nei paesi meno sviluppati), l'accesso delle PMI ai servizi finanziari e alla tecnologia adatte all'ambiente, l'aumento significativo dell'attività e dell'occupazione della ricerca scientifica ed infine, sempre in particolare per i paesi sviluppati, l'accesso alle tecnologie di informazione e comunicazione, con specifico riferimento al fine di fornire un accesso a Internet 'universale ed economico'.

Gli altri obiettivi incrociano, in una logica a rete, le finalità promosse. Proprio la particolare rilevanza dell'interconnessione tra gli obiettivi e la visione unitaria ne rappresenta il nucleo centrale, che brevemente richiamiamo. L'obiettivo 1 si pone l'ambizioso obiettivo di assicurare l'accesso alle risorse economiche, il 2 il raddoppio della produttività agricola, l'aumento degli investimenti sempre nel rispetto delle finalità di tutela dell'ambiente, il 4 il fine di assicurare l'educazione di qualità e accessibile a tutti. L'obiettivo 5 è riferito all'ottenimento di una parità di genere, anche a livello decisionale e di leadership e di rafforzo delle tecnologie 
abilitanti, il 6 e il 7 sono volti a garantire l'accesso universale all'acqua e alle strutture igienico-sanitarie e l'accesso a sistemi di energia economici e affidabili.

Lobiettivo 10 è finalizzato alla riduzione delle diseguaglianze fra nazioni e entro le nazioni rimuovendo al tempo stesso le discriminazioni, l'11 si riferisce allo sviluppo di un'urbanizzazione anche essa inclusiva e sostenibile, il 12 il rispetto di modelli di produzione e di consumo sostenibile, il 13 la promozione di azioni di combattimento del cambiamento climatico e quelli successivi il sostegno delle risorse marine, la protezione dell'uso sostenibile dell'ecosistema, la promozione di società pacifiche e l'accesso alla giustizia al fine di creare istituzioni efficaci; infine il rafforzamento del partenariato mondiale per lo sviluppo sostenibile. Obiettivo, questo, che assume una valenza di norma di chiusura in cui sono convogliate quasi tutte le tematiche indicate prese in conto sotto l'ottica delle istituzioni volte a sostenere l'agenda con azioni sistemiche politiche, tra i quali l'aspetto fondamentale dei monitoraggio e della responsabilità sui dati che devono essere "di alta qualità, immediati e affidabili andando oltre il profitto, il genere, l'età, la razzia, l'etnia, lo stato migratorio, la disabilità, la posizione geografica e altre caratteristiche rilevanti nel contesto nazionale".

Seguono poi le due sezioni, rilevanti tanto quanto - se non di più - quelle indicanti i principi, dedicate all'attuazione e al monitoraggio e verifica degli obiettivi di 'trasformazione del mondo' legati all'agenda.

Il mese di settembre 2015 in cui si colloca l'Agenda 2030 si situa tra due eventi che ne mostrano la collocazione storica precisa: l'enciclica Laudato si' di Papa Francesco, che la ha preceduta di pochi mesi, e la conferenza sull'ambiente che ha portato all'accordo di Parigi, che la ha invece seguita di pochi mesi e ne rappresenta di fatto la prima attuazione, inserendosi in un percorso che muove dall'agenda Onu 21, successiva alla Conferenza di Rio de Janeiro del 1992, fino al Protocollo di Kyoto e alla Dichiarazione del Millennio e i suoi otto obiettivi, di cui l'Agenda 2030 è ideale continuazione, a seguito della Conferenza di Rio + 20 del 2012.

La concentrazione da parte delle politiche mondiali sugli obiettivi di questa visione trasformativa e utopica è stata brutalmente distratta da due crisi globali di portata inattesa, la prima finanziaria a seguito degli avvenimenti del 2008, 
la seconda riferita alla pandemia che stiamo attraversando, in un momento i cui primi target individuati per fornire parametri concreti alla visione utopica dovevano già essere raggiunti, il 2020.

La scadenza del 2030 vede l'indicazione di un'utopia da promuovere e realizzare che intende contrapporsi a previsioni di un vero e proprio crollo del modello di sviluppo occidentale, non considerato più sostenibile per i prossimi decenni ${ }^{1}$, come sostiene il portavoce dell'ASvIS, l'Agenzia Italiana per lo Sviluppo Sostenibile, Enrico Giovannini. Ognuno degli obiettivi corrisponde a una criticità rilevata come ampiamente prevedibile sulla base del modello adottato nel corso del xx secolo e un disegno pensato su scala mondiale in seguito alla venir meno della contrapposizione tra il blocco occidentale liberaldemocratico e quello dei paesi comunisti nel 1989.

Il linguaggio di partenza con il quale l'Agenda si confronta, come si è visto, è il PIL inteso come strumento di misurazione del benessere, che si auspica crescente per i paesi sviluppati, e in forte aumento per $\mathrm{i}$ paesi in via di sviluppo. Questa scelta, anche a fronte di critiche radicali di Sen e di Nussbaum ${ }^{2}$ in relazione alla scelta di questo strumento di misurazione, rinvia ancora a una concezione ispirata a uno sviluppo lineare volto alla massimizzazione del PIL, che deve essere però letto come base di partenza per lo spostamento verso lo sviluppo di un modello più complesso di economia circolare.

La scelta di ripetere e ripetere gli obiettivi in ogni luogo possibile e diffonderne la visione è al tempo steso una strategia normativa e comunicativa, che ha per l'obiettivo la trasformazione di una cultura globale indirizzata verso altre visioni che rischiano di apparire prevalenti. Proprio per questo la ripetizione non è superflua: perché, come nota

1. E. Giovannini, Lutopia sostenibile, Laterza, Roma-Bari 2018.

2. Mi limito a citare M. Nussbaum, Creare capacità. Liberarsi dalla dittatura del PIL, il Mulino, Bologna 2012 e Id., Non per profitto. Perchéle democrazie hanno bisogno della cultura umanistica, il Mulino, Bologna 2013. Segnalo anche il volume specificamente dedicato alla teoria della giustizia, Le nuove frontiere della giustizia. Disabilità, nazionalità, appartenenza di specie, il Mulino, Bologna 2007, che approfondisce il quadro riferito alla Convenzione Onu per i diritti delle Persone con Disabilità del 2006, che mi pare rilevante per intendere, mi pare, in particolare l'obiettivo 3, e la rilevanza del tema della disabilità, che non appare particolarmente sottolineato, come vedremo. 
Cavalli nell'introduzione agli Obiettivi di Sviluppo Sostenibile della Fondazione Eni Enrico Mattei, gli obiettivi appaiono molto lontani dalla situazione esistente, in quanto, ad esempio, ancora oggi «circa 700 milioni di persone vivono ancora al di sotto della soglia di povertà definita dalla Banca Mondiale, e molte altre vivono ancora di privazioni»e, ancora, «molte società hanno registrato un aumento della disuguaglianza, pur avendo raggiunto un progresso economico. Inoltre, il mondo intero affronta minace ambientali, dai cambiamenti climatici indotti alla perdita di biodiversità. Infine scarsa capacità di governance, corruzione e conflitti affliggono gran parte del mondo» ${ }^{3}$. Non si deve pertanto intendere la scelta di una visione ambiziosa e di un linguaggio correlato come manifestazione di una utopistica fuga dalla realtà, ma come tecnica di comunicazione normativa retoricamente efficace per promuovere una visione globale comune in grado di influenzare i comportamenti degli individui, delle diverse culture, dei governanti, imponendo una 'lingua comune dei diritti' da cui sia impossibile discostarsi nell'argomentazione razionale e nel confronto. Come nota ancora Cavalli, si tratta di un'adozione di principi non semplici che pone gli stati a molteplici sfide di grande complessità, fornendo un quadro normativo condiviso, supportato dal diritto internazionale, volto a promuovere la collaborazione e la mobilitazione di tutti gli attori sociali.

\section{La comunicazione giuridica, la teoria del diritto e l'utopia sostenibile: una prospettiva estetico giuridica}

Con specifico riferimento alla complessa situazione italiana, correttamente Giovannini individua l'origine della necessità della narrazione dell'Agenda 2030 in un clima di sconforto diffuso in occidente a partire dai rischi di crollo dei sistemi economici e sociali di fronte ai pericoli connessi alla globalizzazione da un lato, e dal conseguente ribaltamento all'orizzonte degli equilibri economici mondiali, dalle crisi ecologiche e dei mercati finanziari, infine del terrorismo di matrice religiosa. Un'analisi obiettiva del processo di globalizzazione non dovrebbe dimenticare il fatto che se il 
4. E.W. Böckenförde, Diritto e secolarizzazione. Dallo Stato moderno all'Europa unita, Laterza, Roma-Bari 2010, p. 108.

5. P.A. Sequeri, Il sensibile e l'inatteso. Lezioni di estetica teologica, Queriniana, Brescia 2016, p. 124. nomica si è verificata ed è osservabile in seguito alla caduta del sistema comunista (con la rilevante eccezione politica della Cina, però, come vedremo). Appare fuori di dubbio che tale evoluzione ha fatto emergere nuovi rilevanti problemi concernenti l'emergere di nuove diseguaglianze, la non presa in considerazione delle esternalità negative $\mathrm{e}$ il diffondersi di un individualismo distruttivo delle basi stesse delle società occidentali, con pesanti conseguenze riferibili alla sopravvivenza delle stesse democrazie, quasi a rendere innegabile il celebre dilemma di Böckenforde. Il paradosso si riferiva al dato osservabile che lo Stato liberale, secolarizzato, vive di presupposti normativi che antropologicamente non appare più in grado di garantire proprio in seguito allo sviluppo della libertà individuale che ne caratterizza il sistema e ne assicura il funzionamento ${ }^{4}$. Inutile osservare come questa notazione oggi risuoni quasi profetica, ai tempi di una pandemia in cui gli Stati meno attenti al rispetto dei diritti individuali riescono ad adottare politiche più efficaci delle democrazie, almeno dal punto di vista dei risultati. Si tratta però di comprendere se un simile problema non si ponga anche in relazione ai rischi che il modello di sviluppo orientato dal predominio della tecnologia non ponga un simile paradosso, come ha recentemente rilevato il teologo Pierangelo Sequeri, riprendendo la frase del costituzionalista tedesco: «la scienza moderna gronda di metafisica. Nel parafrasare Böckenförde la scienza moderna si regge su presupposti fenomenologici e normativi che non è in grado di garantire fenomenologicamente e normativamente ${ }^{5}$. Torneremo sul punto in relazione alle analisi di Zuboff e di Stiegler nella parte conclusiva dell'articolo.

Giovannini, senza intendere essere considerato sostenitore del modello della decrescita felice, interpreta l'utopia 
sostenibile dell'Onu, e la conseguente narrativa che ne discende, come uno degli elementi difficilmente negabili, ove si consideri evidente che «una crescita annuale del Pil del $2 \%$, in presenza della nuova rivoluzione industriale basata su una massiccia introduzione dell'automazione nei processi produttivi non solo di tipo manifatturiero, non sarebbe in grado di assorbire la disoccupazione e la povertà già presente in Italia e in Europa, figuriamoci quella conseguente a un aumento della popolazione derivante dall'immigrazione" 6 .

Ci troviamo spesso di fronte, nel contemporaneo agone politico dei paesi occidentali, a strategie comunicative politiche assai differenti, che costruiscono scenari catastrofisti, non nel senso virtuoso indicato da Dupuy e legato in qualche modo alla logica che ispira il principio di precauzione (o, se si vuole, dalla narrazione del libro biblico di Giona $)^{7}$, ma come logica di fondo che ispira una strategia elettorale e di opposizione politica volta a strumentalizzare le paure degli elettori. Senza peraltro poter presupporre la razionalità dell'elettorato nella capacità di scelte concorrenti circa le strategie da adottare ${ }^{8}$. In questo senso è da intendere la narrativa e la comunicazione messa in campo dall'agenda 2030, richiesta da un sempre maggior numero di organizzazioni, inclusi leader del mondo economico, politico, mediatico e accademico, ma anche da molti singoli individui.

Questa narrativa differente dovrebbe essere in grado di oltrepassare il quadro fornito dalle nozioni di sostenibilità e di compliance al fine di promuovere una visione del mondo totalmente centrata sulla salvaguardia dell'umanità e del pianeta, non limitata al principio di non recar danno, ma volta al miglioramento sistematico e continuo di tutti i membri dalla società globale come del mondo naturale,

6. E. Giovannini, Lutopia sostenibile cit., e book, posizione 1279.

7. In particolare nel capitolo 'Prevedere l'avvenire per cambiarlo. (Giona contro Jonas)' del libro da me tradotto J.-P. Dupuy, Per un catastrofismo illuminato. Quando l'impossibile è certo, Medusa, Milano 2011, pp. 137 sgg.

8. Un'analisi accurato dei paradossi del voto in un altro libro illuminante di J.-P. Dupuy, Avevamo dimenticato il male? Pensare la politica dopo l'11 settembre Giappichelli, Torino 2010. 
sulla base dei due principi del rispetto della dignità umana ${ }^{9}$ e dell'intero ecosistema, come indica Kuenkel ${ }^{10}$.

Con specifico riferimento all'Italia, un indubbio apporto alla nuova visione, come è stato proposto, sarebbe una modifica costituzionale in grado di aggiornare il quadro del testo fondamentale alla logica inclusiva che muove l'agenda, inserendo un comma all'articolo 3 della Costituzione, che potrebbe recitare "La Repubblica promuove le condizioni di uno sviluppo sostenibile, anche nell'interesse delle generazioni future". Le proposte di riforma testuale, formulate sempre ne Lutopia sostenibile, non si limiterebbero a questo, ma integrerebbero anche l'art. 9 e l'art. 2 del testo fondatore della Repubblica ${ }^{11}$.

Senza dubbio l'inserimento nella Costituzione italiana dell'orizzonte innescato dalla 'nuova visione' promossa dall'agenda 2030 rappresenterebbe un atto di grande valore simbolico e politico, che sarebbe volto, secondo Giovannini, anche a riaprire un dialogo tra le forze politiche sull'ammodernamento della Carta Costituzionale, pur se la proposta di Giovannini si riferiva a un momento storicopolitico preciso.

Se tuttavia gli elementi cruciali individuati per consentire la trasformazione di un sistema verso lo sviluppo sostenibile sono l'innovazione e l'adozione di tecnologie adeguate, una governance all'altezza della gestione complessa del sistema che ne risulterebbe, e infine un cambiamento profondo della cultura e della mentalità individuale, la proposta sembra incorrere in una qualche petizione di principio. Un circuito virtuoso di questo tipo, infatti, presupporrebbe già l'esistenza di almeno due degli obiettivi necessari (una cultura nuova, una governance politica adeguata), proprio l'elemento

9. Per una lettura antropologica del principio giuridico della dignità che si proponga di oltrepassare le differenti visioni del principio, e i conflitti interpretativi da esso generato, mi permetto di rinviare a P. Heritier, La dignità disabile. Estetica giuridica del dono e dello scambio, Dehoniane, Bologna 2014.

10. P. Kuenkel, Stewarding Sustainability Transformation. An Emerging Theory and Practice of Sdg Implementation - A Report to the Club of Rome, Cham, Springer 2019, in particolare nel paragrafo 'A New Narrative for a Sustainable World', pp. 5 sgg.

11. Aggiungendo all'art. 9 «tutela l'ambiente e promuove le altre condizioni di uno sviluppo sostenibile anche nell'interesse delle future generazioni» e all'art. 2, aggiungendo similmente nel testo dell'articolo il riferimento ai diritti delle generazioni future e allo sviluppo sostenibile. E. Giovannini, L’utopia sostenibile cit., posizione 1342. 
che ha invece impedito il processo di riforme costituzionali auspicato fin dalla Commissione Bozzi nei primi anni ottanta e costituisce in realtà uno dei problemi cui l'Onu intende rimediare mediante l'adozione del testo.

Colgo l'occasione di questa proposta per inserire incidentalmente un altro tema, che è la ridotta considerazione, all'interno della stessa agenda 2030, del riferimento all'inclusione dei disabili, uno dei luoghi in cui più efficacemente la progettazione inclusiva ha trovato considerazione, all'interno dei testi dell'Onu, e che contempla in particolare una assai interessante definizione, dal punto di vista della tecnica costituzionale, della disabilità come fenomeno in continua evoluzione e definito da un modello sociale, e non medico. La Convenzione per i diritti delle persone con disabilità (CPRD) definisce infatti la disabilità come categoria relazione e culturale, riferibile nell'articolo 1 al comma secondo che ne precisa lo scopo, a «minorazioni fisiche, mentali, intellettuali o sensoriali a lungo termine che in interazione con varie barriere» possono impedire la piena ed effettiva partecipazione dei disabili nella società su una base di eguaglianza con gli altri. Questo testo è straordinariamente avanzato dal punto di vista della tecnica definitoria, perché invece di fissare in un quadro "statico" un fenomeno, ne riconosce la natura "relazionale" e "sistemica". Il fenomeno "disabilità", in altre parole, non è una caratteristica della persona, ma dell'interazione tra persona e ambiente. Appare allora chiaro come la nuova visione promossa dall'agenda sia interessante perché estende e generalizza la necessità di un modello relazionale simile ai temi dello sviluppo sostenibile e dell'ecologia. Il punto solleverebbe qui problemi di teoria generale del diritto che non possiamo che accennare.

Classicamente, infatti, la teoria pura del diritto di Kelsen introduce una dimensione nomo-dinamica nell'ordinamento giuridico inserendovi la teoria della validità delle norme pensata in relazione a una gerarchia delle fonti del diritto logicamente controllabile, provando a trovare così nella supremazia della norma statale una soluzione al conflitto di valori proprio del dibattito giusnaturalistico sul fondamento del diritto, dottrina imputabile di individuare sempre valori diversi alla nozione di fondamento (pace, libertà, sicurezza, ad esempio nel diversi sistemi dei grandi giusnaturalisti 
classici ${ }^{12}$. Enrico di Robilant, nella sua prospettiva cattolico liberale, provava invece a porre il problema del nesso tra tecnologia e norma invertendo i termini classici del positivismo di Bobbio: a essere considerata "statica" non era la prospettiva valoriale del diritto naturale, ma la prospettiva positivistica kelseniana, incapace di cogliere il "movimento" e la dinamica propria della società complesse, in particolare in riferimento al mondo dell'economia e dell'innovazione tecnologica. Il gioco dell'inversione tra ordinamento normativo statico (proprio del diritto positivo incapace di seguire l'evoluzione economica e tecnologica della società complessa (o tecnologicamente avanzata) e ordinamento economicotecnologico dinamico (soggiacente ai fenomeni dell'ordine spontaneo esteso oggi ascritto al processo di globalizzazione dei mercati) precisava il problema epistemologico proprio del diritto positivo statale e della definizione della norma, in quanto legato alla sanzione propria dell'accostamento kelseniano e bobbiano ${ }^{13}$, nel suo vano inseguire i sistemi economici, e della società industriale o tecnologicamente avanzata, comunemente assunti come a-valoriali.

Ad essere 'statica' è infatti la necessità di fissare un'evoluzione sociale e tecnologica in una norma, possibile solo in società non industriali e tecnologicamente complesse, ma tradizionali o rurali. La norma di origine statuale non può che rincorrere invece l'evoluzione tecnologica propria di una società complessa: proprio su questo snodo teorico interviene il disegno complessivo dell'agenda.

Appare chiaro come il dispositivo ricercato da Giovannini che individua tre fattori centrali per il cambiamento come la disponibilità di tecnologie adeguate, una altrettanto adeguata governance della complessità e la necessità di una differente cultura incontri uno dei problemi di fondo della concezione moderna del diritto: la riduzione del diritto a norma intesa come comando, à la Hobbes (non importa se nella versione decisionista Schmittiana o in quella positivista Kelseniana).

12. Mi limito a far qui riferimento a un testo classico come N. Bobbio, Teoria generale del diritto, Giappichelli, Torino 1993.

13. Ad esempio, in E. di Robilant, Diritto e ordine. Appunti per il corso di filosofia del diritto 1997-98, Giappichelli, Torino 1998. Si veda anche E. di Robilant (a cura di), Libertà e società tecnologica avanzata, Longanesi, Milano 1987. 
In una lettura estetico giuridica, non può certo essere ridimensionato il valore simbolico dell'inserimento in un testo costituzionale o di diritto internazionale di un principio da tutelare, elemento posto alla base della richiesta di Giovannini. Si tratta però di capire come questo possa avvenire concretamente, in una situazione generalizzata di crisi sistemica della democrazia nei paesi occidentali, proprio alla luce delle critiche espresse dall'autore (in particolare, inadeguatezza e mancanza di razionalità degli elettori). L'esistenza di una nuova cultura popolare diffusa rappresenta al tempo stesso l'obiettivo della nuova visione dell'agenda (e del nuovo linguaggio normativo che ne discende), e la condizione della sua attuazione concreta, sia pure entro un contesto di 'complessità' che richiede gli altri due fattori: una governance della complessità adeguata e tecnologie adeguate.

La prospettiva che il ragionamento sottintende, prima definita "petizione di principio" è esattamente il ruolo che assume 'l'utopia'. L'utopia è il buco nero che consentirebbe la risoluzione magica di questo cortocircuito logico-temporale. L'utopia annulla, sia pur funzionalmente, la temporalità intesa come scorrere lineare del tempo (e della storia). Il modello dello statuto del discorso utopico, tuttavia, non appare solo epistemologico, ma anche metafisico, come chiarisce Dupuy.

Proprio l'adozione del principio di precauzione in tema ambientale a seguito della Conferenza di Rio de Janeiro del 1992 indica la questione. Il modello del catastrofismo illuminato di Dupuy chiarisce la natura contraddittoria dell'enunciazione (riferendosi alla profezia piuttosto che l'utopia, ma lo schema non appare subire una significativa modificazione): a fronte di un'incertezza conoscitiva di partenza, essa deve essere creduta vera per potersi realizzare, contraddicendo e opponendosi alla logica della 'prevenzione', che segue un funzionamento diverso, a partire dall'individuazione di un rischio invece conoscibile e dunque affrontabile.

Come ricorda Dupuy, «se avesse seguito i consigli pressanti di von Neumann e di Bertrand Russell, l'America avrebbe pietrificato l'Unione Sovietica sotto un tappeto di bombe all'idrogeno. Essa non l'ha fatto e, magari, la rappresentazione che essa avrebbe potuto farlo ha contribuito 
alla pace del mondo. Nella metafisica della prevenzione, questo possibile che sarebbe stato l'inverno nucleare resta, in ogni caso, per sempre un possibile, non nel senso in cui potrebbe ancora oggi essere attualizzato, ma nel senso in cui resterà per sempre vero che avrebbe potuto essere realizzato» ${ }^{14}$. Oltre l'esempio, tornando alla logica del discorso (utopico, profetico) l'epistemologo di Stanford precisa: «ogni volta che utilizziamo il futuro dell'indicativo per affermare qualche cosa - per esempio "le torri del World Trade Center saranno ricostituite prima della fine del secolo" - presupponiamo il principio della realtà dell'avvenire. Per convenzione linguistica, la proposizione che esprimiamo con questa frase è in effetti vera o falsa nel momento in cui la facciamo se e solo se sarà vero, prima della fine del secolo, che le torri del World Trade Center saranno ricostituite. Ogni volta che facciamo una previsione, diamo una realtà all'avvenire» ${ }^{15}$.

Semplificando molto il ragionamento epistemologicometafisico-linguistico di Dupuy (per cui rinvio alla lettura del libro sul catastrofismo, o almeno alla parte in cui viene analizzata la struttura del discorso del profeta biblico Giona), la concezione del diritto moderno tende a eliminare precisamente il problema della norma, considerandola necessariamente vera: non a caso la teoria validità della norma sottintende sempre il problema della sua efficacia, frutto di infinite discussioni in teoria del diritto, da un secolo. Lefficacia (controversa) è, per così dire, l'ombra della validità.

Il problema concernete il linguaggio utopico dell'agenda è che non sappiamo (non possiamo affatto sapere) se gli obiettivi dell'agenda 2030 saranno realizzati. Siamo anzi praticamente sicuri che non lo saranno, o lo saranno solo in parte. Il fatto è però che se non iniziamo a parlarne, discutere come se fossero realizzabili molto difficilmente lo potranno essere. Non nel 2030, forse mai. Anzi probabilmente osserveremo una direzione dello sviluppo della società in una direzione esattamente opposta, verso una società in cui cresceranno le diseguaglianze, aumenteranno i conflitti e le guerre e lo sfruttamento del pianeta. 

quella dell'Agenda 2030 è a sua volta già il frutto di uno sviluppo virtuoso, in grado di innescare una trasformazione significativa della cultura e delle prassi. La norma ha uno statuto anche necessariamente mitico e utopico, sempre, inevitabilmente, e in questo senso deve essere inteso il richiamo all'utopia. All'interno dei testi normativi occorre inserire il riconoscimento e l'esplicitazione di questa dinamica antropologica soggiacente alla comunicazione e alla capacità della fede o della fiducia dell'uomo di trasformare il reale mediante il riconoscimento di categorie relazionali, in grado di trasformarsi rapidamente come l'evoluzione tecnologica e non con i tempi lunghi del diritto e della decisione politica ${ }^{16}$. Torniamo alla definizione della disabilità come "minorazione in relazione a barriere": oltre il prevalere del modello medico, per giungere a quella fondamentale dichiarazione, si sono impiegati decenni di disability studies, di proteste e azioni di associazioni che hanno compiuto battaglie civili, e che hanno condotto a modificazioni terminologiche lente e non necessariamente universali (in Francia, ad esempio, la parola disabile che ha sostituito il vecchio termine handicappato, ancora non esiste).

La nuova visione promossa dall'Agenda 2030 si inserisce nella stessa logica e in una concezione del diritto che ritorna, oltre a Hobbes e al positivismo keleseniano (ma anche oltre al realismo giuridico nordamericano) a dare spazio al "consiglio", come forma normativa posta accanto al comando-norma, come il diritto canonico medioevale ha sempre conosciuto. Come notava Bobbio il capitolo sulla nozione di consiglio, abituale nei trattati di diritto naturale, è scomparso dai manuali di teoria generale del diritto, al più «relegato in qualche angolo del diritto canonico» ${ }^{17}$. Nella concezione assolutista di Hobbes la delimitazione del comando dal non comando diviene addirittura il criterio distintivo tra quel che è giuridico e quel che non lo $\grave{e}^{18}$. Quel che però Bobbio

16. R. Cubeddu, Il tempo della politica e dei diritti, IBL, Milano 2013.

17. N. Bobbio, Comandi e consigli, in Studi per una teoria generale del diritto, Giappichelli, Torino 2012, pp. 39-64, p. 39.

18. N. Bobbio, Comandi e consigli cit., p. 43. Per un'analisi critica, P. Heritier, Estetica giuridica, vol. II. Il fondamento funzionale del diritto positivo a partire da Legendre, Giappichelli, Torino 2012. 
non ha potuto vedere, è il problema della necessità della reintroduzione delle tecniche di "consiglio" entro il quadro del positivismo. L'intero dibattito sulla distinzione tra principi e norme, che ha occupato la gran parte dei dibattiti teorici della seconda metà del xx secolo ruotano intorno a questo problema: la giuridicità della sfera del consiglio e dunque la rilevanza delle tecniche di comunicazione ai fini della costruzione di una Bildung, della trasformazione graduale e progressiva di una cultura come fattore di mutamento auspicato, "utopia" da credere e non evidenza di una realtà già realizzata in quanto semplicemente scritta in una norma. I nomi à la page di questa "resurrezione" del consiglio risuonano per lo più in lingua inglese, come se introducessero un elemento di novità: soft law, governance, best practices $^{19}$. In realtà il problema teorico che queste nozioni riaprono è esattamente il tema del consiglio e delle forme della comunicazione del diritto in relazione alla formazione della coscienza civile e sociale, in un contesto di declino della figura di razionalità sotto l'impatto dei processi di disindividualizzazione ${ }^{20}$ e dei rischi di trasformazione digitale imposti dal "capitalismo della sorveglianza" di Google e Facebook, come vedremo.

Occorre però tornare brevemente al tema della disabilità all'interno dell'agenda 2030 a partire da un articolo di Lockwood e Tardi, che chiarisce alcuni aspetti critici dell'agenda. Il documento finale inserisce 11 riferimenti alle persone con disabilità, negli ambiti più vari, dall'educazione al lavoro, dalla riduzione delle diseguaglianze alla disaggregazione dei dati, che si sommano ad altri riferimenti alle persone vulnerabili. Tuttavia, l'impressione che si sarebbe potuto inserire nel nucleo centrale dell'agenda il tema della progettazione inclusiva permane: come notano Loockwood e Tardi, per le persone con disabilità «l'accessibilità dovrebbe diventare un criterio centrale nello sviluppo di ogni genere di attività e il concetto di sviluppo inclusivo

19. Nella sterminata bibliografia in tema, J. Lenoble, M. Maesschalck, Democracy, Law and Governance, Ashgate, 2010; con riferimento al dibattito italiano, A. Andronico, Viaggio al termine del diritto: saggio sulla governance, Giappichelli 2012.

20. Per un'analisi del problema, si veda P. Sequeri, L'umano alla prova. Soggetto, identità, limite, Vita e Pensiero, Milano 2002; P. Sequeri, La cruna dell'ego. Uscire dal monoteismo del sé, Vita e Pensiero, Milano 2017. 
precisa che le persone con disabilità “devono essere incluse in tutte le fasi dei programmi di sviluppo, comprese la pianificazione, il design, l'implementazione e la valutazione» ${ }^{21}$. L'accostamento "relazionale" al fenomeno della disabilità che la CPRD statuisce appare utile nel dare forma precisa al riferimento alla dignità umana: dignità significa progettazione inclusiva, e tale progettazione è la vera questione soggiacente la nuova visione, in relazione ai temi dello sviluppo, dell'uso delle nuove tecnologie, del lavoro, dell'industrializzazione.

3. Visioni ottimistiche e pessimistiche su industrializzazione sostenibile e uso delle tecnologie: big data, innovazione sociale, capitalismo della sorveglianza

Una volta chiarito il senso del linguaggio comunicativo-normativo dell'agenda all'interno del processo di formazione di una nuova visione globale da diffondere, appare allora chiaro che la tensione che può essere individuata muove proprio dalla mancanza di una piena integrazione del modello inclusivo formulato in testi come la Cprd e gli obiettivi dell'agenda, che sembrano non portare fino alle estreme conseguenze la scelta tra un modello pienamente inclusivo dei disabili, dei poveri, in generale dei discriminati e del modello di sviluppo di paesi meno sviluppati confrontati a quelli tecnologicamente avanzati. Problema che mi sembra riferibile a una visione teorica non ancora sufficientemente chiarita, prima di tutto a livello teorico nel confronto tra saperi e priva di una visione filosofica coerente.

La soluzione di questa tensione mi pare affidata, infatti, più che a un'impostazione teorica, al concreto svolgersi delle attività di messa in opera e di progettazione dei principi, in cui proprio la partecipazione di tutti gli attori coinvolti nel processo può fornire risposte concrete a questo problema di fondo. Si tratta della questione già indicata come la governance complessa dei processi di attuazione, in cui il conflitto tra logica dell'inclusione e logica dello sviluppo competitivo esistente può condurre a tensioni non superficiali.

Il nucleo del problema individuato riguarda però il terzo elemento indicato da Giovannini accanto a governance 
22. E. Giovannini, La rivoluzione dei big data a sostegno dell'Agenda 2030, «Equilibri», 1 (2016), p. 64.

23. Ivi, p. 65.

24. Ivi, p. 68.

25. Come precisa P. Conceição, Fear an Loathing of technological Progress? Leveraging Science and Innovation for the Implementation of the 2030 Agenda for Sustainable Development, in A. Baimenov. P. Liverakos (eds.), Publick Service Excellence in the $21^{\text {st }}$ Century, Palgrave macmillan, Singapore 2019, pp. 31-52. Nello stesso volume, in tema diffusamente anche M. meno avanzati sul piano statistico e analitico» ${ }^{24}$ investendo anche nella formazione di data scientist in grado di integrare la conoscenza dei sistemi di calcolo della probabilità, dell'economia, della sociologia e dell'ecologia con la nuova visione, aggiornata alla "data revolution".

In realtà, visioni diverse mostrano come qualsiasi discorso sull'uso della tecnologia sia sempre necessariamente ambivalente nella storia dell'umanità, incluso quello proprio dell'agenda 2030, come precisa Conceição ${ }^{25}$. Se infatti la prospettiva dell'agenda lega l'uso delle tecnologie dell'intelre ricerca come Horizon 2020, potrebbe aiutare 11 mondo intedella complessità e cambiamento della cultura diffusa, vale

Come lo stesso autore sostiene in un articolo specificamente dedicato al problema, l'attenzione sul problema dei
dati statistici disponibili è uno degli elementi che l'agenda chiarisce con forza, collegando il suo accostamento a quello della "data revolution" in corso e dell'uso dell'intelligenza artificiale e della robotica nel processo di innovazione tecnologica. Limitandoci ai dati, essi, in termini di quantità e qualità, non appaiono affatto soddisfacenti: nonostante considerevoli miglioramenti, «vengono ancora ignorati fenomeni che riguardano intere popolazioni, così come non
vengono misurati statisticamente importanti aspetti della vita umana e delle condizioni del Pianeta» ${ }^{22}$, il che implica violare la dichiarazione universale dei diritti dell'uomo sul piano individuale e continuare a percorrere la direzione inversa a quella indicata dall'Agenda, quella del degrado ambientale. Se i dati rappresentano infatti «la linfa vitale dei processi decisionali» ${ }^{23}$ persistono vecchie e nuove forme di diseguaglianza fra nazioni povere e ricche di dati, e rischi significativi di abusi e di controllo dei dati. In questo senso 
ligenza artificiale e della rivoluzione dei big data non solo agli obiettivi di aumento dell'efficienza della produttività e del profitto delle imprese, ma anche della lotta al cambiamento climatico e al miglioramento della formazione e della salute, occorre tuttavia muovere da un modello non deterministico dell'impatto delle muove tecnologie, ma antropologicamente più complesso, che tenga in conto che ogni tecnologia è anch'essa, proprio come la nozione di disabilità, da intendere in senso relazionale e ambientale. In questo senso la nuova narrativa sullo sviluppo promossa dall'agenda si indirizza in senso contrario a quell'altra narrativa, oggi assai diffusa nei paesi sviluppati e tecnologicamente avanzati, che considera il tecno-determinismo e l'adozione delle nuove tecnologie come un fattore inevitabile e necessario ad ogni costo, fino a divenire, più che un'utopia, un vero e proprio mito, se non un credo religioso, come nel caso dei seguaci di vere e proprie "sette" legate al transumanesimo.

Il riconoscimento dell'ambivalenza dell'uso delle tecnologie, dunque la possibilità di usi disumanizzanti delle tecnologie (compresa l'intelligenza artificiale) sul piano dei rapporti di lavoro, e della creazione di nuovi 'poveri digitali' nelle società sviluppate mi sembra il punto centrale intorno a cui si deve concentrare la critica alla lettura ottimistica della visione dell'Agenda e alla pratica della sua attuazione, che deve riconoscere l'elemento umano come centrale nella recezione graduale delle nuove tecnologie, liberandosi dalla influenza delle nuove mitologie tecno-deterministiche e dalla retorica dei suoi effetti sul vivere civile. Un caso rilevante in relazione all'ambito giuridico è fornito dalle nuove tecnologie blockchain per una rivoluzione nell'ambito dei contratti, dai confini ancora assai diversamente valutate ${ }^{26}$.

Everest-Philips, Big-Data Driven Public Service in the Twenty-First Century: the Politics of Big Data, pp. 275-318.

26. In tema di tecnologie blockchain e agenda 2030, A.M. Gomez-Trujillo, J. VelezOcampo, M.A. Gonzalez Perez Trust, Transparency, and Technology: Blockchain and Its Relevance in the Context of the Agenda 2030, in M.A. Gonzalez Perez, D.E. Floriani (eds.), The Palgrave Handbook of Corporate Sustainability in the Digital Era, Palgrave Macmillan, Cham 2020, pp. 561-580. Per valutazioni diverse sul rapporto tra tecnologie blockchain e diritto: A. Santosuosso, Intelligenza artificiale e diritto. Perché le tecnologie di IA sono una grande opportunità per il diritto, Mondadori università, Firenze, 2020, pp. 121-148; A. Garapon, A. Lassègue, Justice digitale, PUF, Paris 2018, pp. 139-163. 
Simili ambiguità sembrano persistere nel riferimento al concetto di sviluppo economico nel contesto dell'agenda. Esso di per sé non significa affatto la creazione automatica di società volte all'implementazione di uno sviluppo durevole a medio lungo termine, nel quadro di un'attenzione alla promozione dell'accrescimento delle potenzialità umane in un modo dignitoso e delle esigenze di conservazione dell'ambiente, come riconoscono Harguchi e Kitaoka ${ }^{27}$. Se infatti è riconosciuto che una trasformazione strutturale dell'industria appare necessaria per creare un modello di sviluppo stabile e lavori dignitosi in grado di sradicare la povertà, conducendo a livelli sostenibili di opportunità per tutti, la visione dell'agenda 2030 cerca di integrare l'uso intelligente dell'industrializzazione (smart industrialization) in una visione unitaria, pur se il modello ipotizzabile per paesi in via di sviluppo e paesi sviluppati permane tuttavia differente. Nei paesi sviluppati si tratta di mantenere il livello di vita migliorandone la qualità, nei paesi in via di sviluppo l'obbiettivo è incentrato su un modello di sviluppo volto invece a raggiungere obiettivi primari posti oltre il livello di mera sussistenza. L'analisi delle situazioni deve dunque distinguere tra un concetto di smart industrialization per il sostegno di redditi medio-bassi e di redditi sopra la media ed elevati. Inevitabilmente, pertanto, l'accesso ai mercati internazionali in paesi poco sviluppati deve prevedere un'industrializzazione volta a introdurre e mantenere settori primari come le industrie alimentari e tessili, caratterizzate da alto impiego di manodopera e di soddisfazione di bisogni umani primari: permettendo così di mantenere un rapporto soddisfacente tra sviluppo dell'industria manifatturiera e la riduzione dell'ineguaglianza di reddito e della povertà, consentendo un graduale accesso ai mercati internazionali, al di fuori di un modello ancora neocoloniale di sfruttamento delle risorse ${ }^{28}$. Questo modello di sviluppo osservabile sembra però implicare la perdita progressiva di competitività delle industrie dei paesi sviluppati a reddito elevato, tesi pertanto a spostarsi su modelli di sviluppo volti a accrescere la produttività tramite l'uso dell'innovazione 
tecnologica, con conseguenze osservabili sul mercato del lavoro legati ai processi di automazione e di aumento della produttività, innescando rischi di creazione di nuove povertà ed emarginazione sociale.

Una recente analisi della situazione è fornita da un utile volume che discute il prossimo futuro dell'articolazione tra i diversi modelli di sviluppo a livello globale, analizzando prospettive differenti provenienti dall'Africa, dall'Europa e dall'Asia, che in particolare prova a approfondire l'articolazione e l'interazione tra i diversi modelli geografici pensati in un contesto unitario e globale ${ }^{29}$. Limitandoci a qualche cenno relativo all'Europa, una posizione di grande interesse è fornita da Schmiedeknecht, che propone la nozione di innovazione sociale come fattore principale per lo sviluppo di un'imprenditorialità sociale europea in grado di inserirsi virtuosamente nel modello di sviluppo concepito dall'agenda ${ }^{30}$. Il processo di innovazione sociali può essere riferito a un modello ad esempio prendente avvio dalla generazione di idee che individuano bisogni sociali per identificare possibili soluzioni, giungendo poi a testare in pratica la soluzioni individuate ritenute migliori creando prototipi, per occuparsi poi della promozione e della diffusione in scala dell'idea, coordinando eventualmente attori e istituzioni in partnership pubblico-private al fine di consentire l'implementazione del progetto in scala e che, da ultimo, non trascura il continuo monitoraggio e l'adattamento dell'idea all'evoluzione del bisogno ${ }^{31}$. Si tratta naturalmente solo di un accenno a un possibile di modello di imprenditoria sociale in grado di articolare in modo sostenibile le tensioni tra tutela dell'occupazione, competitività internazionale e obiettivi individuati dalla Agenda. Altri articoli nello stesso volume sviluppano aspetti concernenti problemi specifici riferiti al mercato del lavoro e alla competitività fra nazioni innescata dal processo di globalizzazione, che fanno emergere le difficoltà individuabili in ciascuno di questi ambiti.

29. S. O. Idowu, R. Schmidpeter, L. Zu (eds.), The Future of the Sustainable Development Goals. Business Perspectives for Global Development in 2030, Spinger, Cham 2020.

30. M. H. Schmiedeknecht, Social Innovation and Entrepreneurship Supporting the Susainable Development Goals (Sdgs) -Fostering Social Value Creation, in S. O. Idowu, R. Schmidpeter, L. Zu (eds.), The Future of the Sustainable Development Goals., pp. 211-225.

31. Ivi, p. 217. 
Da ultimo, non appare possibile trascurare scenari critici invece totalmente differenti, che forniscono un quadro pessimistico rispetto al modello di sviluppo reale dell'ultimo decennio, riferite in particolare all'evoluzione dello scenario dell'innovazione tecnologica e dei suoi effetti sul mantenimento di un quadro democratico all'interno dei paesi sviluppati. Lanalisi di Zmuda, in particolare ${ }^{32}$, individua gli scenari in cui la competitività innescata tra gli stati nazione ha dato luogo, nel primi due decenni del nuovo millennio, a fenomeni di competizione iniqua, a cui il paradigma neoliberale non sembra aver fornito soluzione, allorché proprio la dimensione globale della visione dell'agenda invita a richiedere un nuovo ruolo regolativo dello stato volto proprio all'indirizzamento della competitività, da finalizzare all'ottenimento di uno sviluppo sostenibile.

Proprio questo ultimo rilievo introduce la necessita di riferirsi, sia pure brevemente, alla critica radicale alla trasformazione del capitalismo dei paesi occidentali avanzati, in particolare degli Stati Uniti, negli anni venti, verso un capitalismo della sorveglianza.

L'analisi accurata di Shosanna Zuboff nel volume balzato recentemente alle cronache in tutto il mondo, Il capitalismo della sorveglianza. Il futuro dell'umanità nell'era dei nuovi poteri, introduce con competenza, per certi versi, una visione del tutto contraria al quadro ottimistico veicolato dall'agenda 2030, individuando nel prepotente emergere di Google, Facebook, Amazon e alle evoluzioni di Microsoft e Apple, ai vertici del potere economico mondiale, un processo di trasformazione epocale verificatosi in poco più di un decennio. Tale visione considera il nesso tra tecnologia e capitalismo come lo scenario di un nuovo Far West, permesso dalla retorica neoliberale piegata agli interessi delle corporation irrispettosi dei diritti (in particolare la privacy digitale) il cui obiettivo è divenuto, tramite gli strumenti del machine learning, la profilazione dell'individuo e la costruzione di un sistema di big data volti ad opprimere, e non certo a promuovere le potenzialità dell'uomo. Nel nuovo quadro, le persone, conclude Zuboff «non vengono più viste dalle 
aziende come impiegati o consumatori: è un dato di fatto d'importanza cruciale alla luce dello storico rapporto tra capitalismo e democrazia ${ }^{33}$, evocante scenari orwelliani e frutto dell'ideologia del tecno-determinismo camuffata da innovazione esclusivamente benefica. Le persone, nell'economia della rete, divengono semplicemente materiale inerte da cui estrarre dati che consentono di accumulare enormi guadagni grazie alla previsione e alla modifica del comportamento umano. Come precisa Zuboff: «ricordiamoci come Marc Zuckerberg si era vantato del fatto che Facebook avrebbe conosciuto ogni libro, ogni canzone o film letto, ascoltata o visto da una persona, sostenendo che i suoi modelli predittivi ci avrebbero detto in quale locale andare al nostro arrivo in una città sconosciuta, dove il bartender ci avrebbe atteso con il nostro cocktail preferito sul bancone» ${ }^{34}$. Questo scenario è certamente uno scenario apparentemente gratificante, o innocuo, ma la realtà soggiacente è del tutto differente: "come ha sostenuto il capo del data science team di Facebook "per la prima volta ci sono abbastanza dati di qualità sulle comunicazioni tra persone». Come ha detto Eric Schmidt di Google nel 2010, "più informazioni ci date su di voi e sui vostri amici, migliore sarà la qualità delle vostre ricerche. Non serve nemmeno che scriviate. Sappiamo dove siete. Sappiamo dove siete stati. Sappiamo più o meno anche a cosa state pensando". Satya Nadella di Microsoft considera indicizzabili e ricercabili tutti gli spazi istituzionali, tutte le persone e i rapporti sociali; tutto può essere il soggetto del riconoscimento del pattern, delle previsioni, delle interruzioni e delle modifiche dei comportamenti attuate dalle macchine ${ }^{35}$. Ogni comportamento, ogni azione è tracciata a insaputa o senza un reale consenso dell'utente, come vedremo, e sfruttata a fini predittivi sulla base di una rappresentazione del futuro dell'umanità totalmente distorta e affermatasi globalmente, nel silenzio regolativo quasi assoluto degli stati (anche se entro l'Unione Europea, e recentemente anche negli Stati

33. S. Zuboff, Il capitalismo della sorveglianza. Il futuro dell'umanità nell'era dei nuovi poteri, Luiss University Press, Roma 2019.

34. Ivi, p. 513.

35. Ivi, pp. 513-514. 
36. Linsieme dei dati predittivi sul comportamento dell'individuo ottenuto tramite la profilazione e l'applicazione delle tecnologie di intelligenza artificiale di trattamento dei dati.

37. S. Zuboff, Il capitalismo cit., p. 235. rende superflue tali relazioni... Sono stati il successo delle dichiarazioni di Google e la strada da essa aperta al dominio dell'apprendimento da parte dei capitalisti della sorveglianza a permettere di sostituire l'operato sociale con l'operato delle macchine. Dalla sua torre di vedetta, Google può osservare quel che prima non era osservabile, e sapere quel che prima non era conoscibile. Di conseguenza, l'azienda può fare quel che prima non era fattibile: bypassare le relazioni sociali a favore di processi informatici automatizzati che impongono comportamenti in grado di farle raggiungere determinati obiettivi economici» ${ }^{37}$.

Inutile proseguire l'analisi, dopo la lunga citazione che mostra l'impatto e la rilevanza della nuova visione tecnodeterminista. La stessa democrazia appare un ostacolo al capitalismo della sorveglianza ed è pertanto considerata un

zione sta mutando).
In questa visione è la stessa nozione di contratto a scomparire: non vi è quella posizione di reciprocità tra consumatore e produttore alla base dell'emergere della democrazia liberale moderna, ma semplicemente di sudditanza: i finti contratti in cui si delega la propria privacy a Google analytics o a società simili non sono, secondo Zuboff, contratti, ma rappresentano piuttosto, «l'annientamento del contratto: un'invenzione meglio definibile come non-contratto. Il non-contratto è parte del più ampio complesso dei mezzi di modifica del comportamento, ed è pertanto necessario per l'esistenza del capitalismo della sorveglianza. Apporta economie di azione facendo leva sul surplus comportamenta$\mathrm{le}^{36}$ per prevenire e proibire azioni alternative, rimpiazzando l'indeterminatezza dei processi sociali con il determinismo dei processi programmati. Non si tratta di automazione della società, come qualcuno potrebbe pensare, ma di sostituzione della società con azioni imposte dalle macchine e determinate da imperativi economico. Il non-contratto non è uno spazio per relazioni contrattuali ma un'esecuzione unilaterale che 
ostacolo da superare nel processo di sviluppo tecnologico figlio di una cultura superata e retrograda.

Se per Zuboff il punto di partenza di questa involuzione originario è il capitalismo di Hayek e Friedman, un'altra analisi, quella del giuslavorista Supiot, individua invece la data centrale nello spiegare la contemporaneità o il prossimo futuro sono invece quelle nozze tra capitalismo e comunismo del 1978. Questo sarebbe il momento centrale per comprendere il nostro prossimo futuro, piuttosto che la 'rivoluzione neoliberale' di Reagan e della Thatcher, che avrebbe permesso di giungere alle forme di totalitarismo strisciante analizzato da Zuboff. In quel contesto, infatti, il riferimento alla democrazia è del tutto superfluo, trattandosi di regimi totalitari, la cui influenza sull'Onu è crescete, peraltro. Il giuslavorista francese vede il passaggio al mercato del comunismo cinese come fattore chiave della periodizzazione storica contemporanea: la cui origine risiede nella governance mediante il calcolo, che ha sostituito il ruolo della politica e dello Stato (e qui l'analisi sembra condividere la posizione di Zuboff). Ne Lo Spirito di Filadelfia. La giustizia sociale dinnanzi al mercato totale, indica come il Mercato proprio della teoria economica ultraliberale si presenti come «un modello d'autoregolazione, del tipo di quello che la Scienza (quella presunta vera) osserva presso gli esseri viventi e che la tecnica (in primo luogo l'informatica) mette in opera nelle macchine ${ }^{38}$. La ragione per la quale pensieri diversi come la sociologia marxista e l'economia ultra liberale convergono è la comune fede in leggi immanenti all'umano che possono essere esportate in ambiti diversi dall'economico. Viene così realizzata l'estensione di concetti di matrice economica (domanda, offerta, concorrenza, prezzo) ad ogni ambito della sfera dell'umano. In questa visione, l'umano finisce per essere considerato come una particella elementare, un mero atomo regolato da dinamiche chimiche che ne possono spiegare il funzionamento, sulla base della metafora dell'uomo macchina, e della macchina orologio: nasce «la credenza in un mondo retto da un calcolo di utilità è succeduta agli scientismi del periodo precedente alla guer-

38. A. Supiot, L'esprit de Philadelphia. La justice sociale face au marché total, Seuil, Paris 2010, p. 75 . 
39. Ivi, p. 76. Ho sviluppato la posizione di Supiot in P. Heritier, La libertà tra teologia e diritto dopo le secolarizzazioni, «Archivio teologico torinese», 1 (2018), pp. 319-339,

40. Per un'analisi sintetica della posizione di Stiegler in relazione al ruolo delle nuove tecnologie di fronte alle humanities, mi permetto di rinviare a P. Heritier, Law and Humanities. Umanesimo e svolta affettiva, in A. Andronico, T. Greco, F. Macioce, Dimensioni del diritto, Giappichelli, Torino 2019, pp. 441-467. caparramento selvaggio da parte delle corporation del digitale dei guadagni proveniente dall'uso dei dati personali, straordinari profitti indebitamente sottratti agli individui ${ }^{40}$ che stanno modificando lo stesso assetto (post-petrolifero) del capitalismo, ove i dati digitali "sono il nuovo petrolio".

Queste diverse analisi mostrano come ben altre narrazioni ampiamente diffuse siano alternative a quella dell'agenda 2030 sul futuro dell'umanità, nella comprensione dell'intricato intreccio tra lavoro, automazione, società, democrazia. Siamo in un vero e proprio periodo di lotte di rappresentazione sul futuro dell'umanità, e l'agenda 2030 è una di queste visioni concorrenti. La narrazione rassicurante dei giganti del digitale è legata a una pratica oppressiva e antidemocratica che procede nella direzione opposta a quella dell'agenda 2030, spesso trascinata, soprattutto nei confronti delle nuove generazioni sempre più culturalmente indifese, dai social network. Quella critica e pessimistica del capitalismo della sorveglianza è invece volta a suscitare uno shock e una reazione e può convergere con quella dell'agenda, aiutando a renderla meno ingenua, individuando con chiarezza i rischi connessi all'uso oppressivo dell'innovazione tecnologica e dell'uso dell'intelligenza artificiale. In quest'epoca di grandi cambiamenti nell'arco del secondo decennio è emerso il sistema del capitalismo della sorveglianza. Occorre forse aggiungere agli obiettivi dell'agenda 2030 da finalizzare all'obiettivo della conversione di quest'uso distorto delle nuove tecnologie, per 
riportarle alla loro relazione possibile con la promozione della dignità umana e lo sviluppo sostenibile. Lobiettivo della liberazione da queste forze oppressive e la loro conversione agli scopi dell'agenda tramite una nuova alleanza tra cittadini consapevoli delle poste in gioco, è la posta in gioco urgente, che attribuisce un nuovo ruolo allo stato nella difesa della democrazia, in quanto soggetti in grado di contrastare la logica di azione delle corporation aggressive, che in pochi anni hanno piegato gli ideali della liberaldemocrazia ai propri interessi politici ed economici. Entro il 2030 quest'obiettivo è forse la principale battaglia all'ordine nel giorno nei paesi occidentali, in cui il ruolo dell'Unione Europea potrà essere centrale. 
III.2. II contributo delle religioni

aA 

Potrebbe sembrare superfluo parlare della responsabilità ebraica per il benessere della società, dal momento che gli storici hanno già da tempo convenuto che le idee ebraiche hanno rappresentato un elemento formativo della civiltà occidentale, e che numerosi ebrei individualmente, specie in tempi moderni, hanno ricoperto un ruolo centrale nel suo sviluppo ${ }^{1}$.

Nel celeberrimo saggio Confrontation Rav Soloveitchik delineava il duplice compito del popolo ebraico, da una parte quello di esseri umani che condividono il destino di Adamo nel suo incontro con la natura, e quello di membri di una comunità che ha mantenuto la propria identità in condizioni avverse ${ }^{2}$. In un saggio, significativamente intitolato Straniero e residente, espressione che riprende le parole di Abramo in Gn. 23,4, Rav Soloveitchik riassume il suo pensiero su questo punto:

Il nostro approccio al mondo esterno è sempre stato ambivalente: collaboriamo con persone appartenenti ad altre

1. G.J. Blidstein, Tikkun Olam, Tradition 29:2, 1995, p. 5.

2. J.B. Soloveitchik, Confrontation, Tradition 6,2, 1964, p. 17. 
fedi in tutti i campi dello sforzo umano, ma nello stesso tempo cerchiamo di preservare la nostra distinta identità, che inevitabilmente comprende aspetti di separazione. Questa è una situazione paradossale. Tuttavia, parafrasando le parole del nostro primo antenato Abramo, noi siamo molto presenti nella più vasta società ed al tempo stesso ci manteniamo stranieri ed esterni nel nostro persistente sforzo di preservare la nostra identità storico-religiosa. ${ }^{3}$

Rav Sacks analizza i primi versi del cap. xII della Genesi, nei quali viene descritto l'inizio del viaggio di Abramo. D. promette (Gn. 12,3 ) che per mezzo di lui tutte le famiglie della terra saranno benedette ${ }^{4}$. Una parte importante della missione di Abramo concerne la sua missione all'interno della società.

In Confrontation, che ha come tema principale quello del rapporto con la fede cristiana, poco prima che vedesse la luce la dichiarazione Nostra Aetate, Rav Soloveitchik pone alcuni punti fermi, che sono tutt'oggi pienamente validi, come l'impegno per il benessere generale e il progresso del genere umano, per la lotta alle malattie e all'alleviamento della sofferenza umana, per la protezione dei diritti umani, per l'aiuto dei bisognosi, ecc. ${ }^{5}$. Come è noto Rav Soloveitchik non era un sostenitore del dialogo di ordine teologico, ma credeva tuttavia che vi fossero delle aree di azione comune che dovrebbero essere indirizzate verso l'esterno: «come combattere il secolarismo, il materialismo, la negazione ateistica della religione e dei valori trascendenti che minacciano l'esistenza di parametri morali all'interno della nostra società» ${ }^{6}$.

Tutto oggi è tremendamente complicato, e non si può pretendere che le religioni, che sono sorte migliaia di anni fa, molto prima della nascita della modernità, possano fornire una risposta puntuale ai problemi attuali; le religioni tuttavia hanno molto da dire circa dove stiamo andando o dove dovremmo andare ${ }^{7}$. Ad esempio, su larga scala, è pos-

3. J.B. Soloveitchik, Riflessioni sull'ebraismo, Firenze 1998, p. 185.

4. Citato in J. Sacks, Tikkun Olam: Orthodoxy's Responsibility to Perfect G.d's World, 1997, in https://advocacy.ou.org/tikkun-olam-orthodoxys-responsibility-to-perfect-g-ds-world/.

5. J.B. Soloveitchik, Confrontation cit., pp. 20-21.

6. Id., Riflessioni cit., p. 185.

7. J. Sacks, La dignità della differenza, Milano 2004, p. 21. 
Vita umana, lavoro e sviluppo tecnologico

sibile indicare il chesed, nella sua accezione fisica e materiale, come un obiettivo concretizzabile su una scala universale ${ }^{8}$.

La necessità di un impegno in questo senso oggi è quanto mai pressante. La politica da sola non è in grado di fornire delle risposte soddisfacenti alle persistenti domande che attanagliano buona parte dell'umanità. Fame, malattia, assenza di libertà fondamentali sono condizioni quantomai diffuse nel mondo contemporaneo. La differenza rispetto al passato è che per via delle recenti innovazioni ne siamo consapevoli. Sino a non molto fa la distanza rispetto a certe situazioni indeboliva la nostra empatia. Oggi non ci sono più distanze, sebbene subentri un senso di impotenza ${ }^{9}$. Non possiamo sostenere di non sapere. La piega assunta dall'economia globale ha privilegiato la libertà di scelta a discapito della ricerca del bene comune ${ }^{10}$, ampliando sempre di più, anziché attenuare, la forbice tra ricchi e poveri. La nuova economia ha la tendenza apparentemente inguaribile a ricompensare un numero ristretto di persone diminuendo le possibilità per la base della scala economica di avere un salario ragionevole ${ }^{11}$.

I sistemi economici devono essere giudicati in base al loro impatto sulla dignità umana. Non è possibile continuare a difendere un ordine che deprime buona parte della popolazione mondiale. Ciò non significa necessariamente abbandonare il mercato globale, ma riconoscere un corpus di valori non derivanti dal mercato che deve essere tenuto in conto nelle nostre scelte sul nostro futuro ${ }^{12}$. La letteratura rabbinica riporta la discussione fra $\mathrm{R}$. Aqivà $\mathrm{e}$ Tinneio Rufo: l'aiuto fornito al povero da parte degli altri esseri umani secondo il romano mette in discussione la provvidenza divina, che di fatto viene resa inutile. R. Aqivà ribatte che $\mathrm{D}$. desidera sì che il povero venga aiutato, ma vuole che gli esseri umani, e presumibilmente non solo gli ebrei, abbiano il merito dell'atto ${ }^{13}$. Si narra che il nonno di Rav Soloveitchik, Chayim di Brisk, interrogato da un

8. G.J. Blidstein, Tikkun cit., p. 13.

9. J. Sacks, La dignità cit., pp. 41-42.

10. Ivi, pp. 20-21.

11. Ivi, p 121.

12. Ivi, p. 26.

13. Si veda G.J. Blidstein, Tikkun cit., p. 13. 
allievo circa il compito di un rabbino, rispose che consiste nel porre rimedio ai motivi di lamentela da parte di coloro che sono abbandonati e soli, proteggere la dignità dei poveri e salvare gli oppressi dalle mani dell'oppressore. Era sempre indebitato e dava la maggior parte del suo stipendio ai poveri. Di inverno lasciava il suo negozio di legna aperto affinché i poveri potessero servirsi senza chiedere. Quando l'amministrazione della comunità si lamentò, perché per via di questo suo comportamento loro stavano perdendo denaro, rispose che in realtà stava risparmiando loro il costo delle spese mediche, perché altrimenti si sarebbe preso una polmonite, dal momento che non avrebbe potuto accendere un fuoco nella propria casa se i poveri stavano congelando ${ }^{14}$.

Rav Sacks cita una norma di Maimonide secondo la quale, quando si tratta di violare lo Shabbat per salvare una vita umana, non lo si fa impiegando non ebrei, bambini o schiavi, ma devono essere i grandi saggi di Israele a violare lo Shabbat in questa circostanza. Perché? Perché salvando una vita si insegna la Torà fondamentale di tutti, che le norme della Torà non sono una forma di vendetta contro il mondo, ma sono volti a portare compassione, pace e gentilezza nel mondo ${ }^{15}$.

La tradizione ebraica ha elaborato il concetto di tzedaqà, un concetto difficile da esprimere secondo le categorie concettuali del mondo occidentale, trovandosi a metà fra la giustizia e la carità. Il livello supremo di tzedaqà, scrive Maimonide, è quello di chi assiste il povero dandogli un dono o un prestito o accettandolo come socio d'affari o aiutandolo a trovare un lavoro, ovvero mettendolo in una situazione in cui può fare a meno dell'aiuto di altre persone ${ }^{16}$.

La sanità è un campo dove le disparità nei servizi resi fra i paesi industrializzati e quelli in via di sviluppo è innegabile, sia in termini di percentuale del Pil investita in tale ambito, sia in termini di spesa pro capite. La relazione fra malattia e povertà è purtroppo lampante e deprimente. Ogni anno milioni di persone muoiono di fame e malattie

14. Citato da N. Zion, Tikkun Olam as the Mastery of Nature: Rabbi J. B. Soloveitchik, 2013, in https://hartman.org.il/Blogs_View.asp?Article_Id=1245\&Cat_Id=413\&Cat_Type $=$

15. Citato in J. Sacks, Tikkun cit..

16. Si veda J. Sacks, La dignità cit., pp. 128-135. 
Vita umana, lavoro e sviluppo tecnologico prevenibili. Non è possibile oggi che centinaia di milioni di persone nel mondo non abbiano accesso all'acqua potabile. Scrive Rav Soloveitchik:

Lumanità dei tempi antichi, che, con degradante impoten$\mathrm{za}$, non riusciva a combattere le malattie e che a moltitudini soccombeva per la febbre gialla o per qualsiasi altro flagello, non poteva rivendicare la dignità. Unicamente l'uomo che costruisce ospedali, che scopre tecniche terapeutiche e che salva vite è benedetto con la dignità. ${ }^{17}$

La velocità e l'estensione delle moderne tecnologie di comunicazione hanno cambiato irreparabilmente le condizioni di vita della maggior parte degli abitanti del pianeta. L'interconnessione è divenuta più immediata di quanto non fosse mai stata in passato ${ }^{18}$. Internet ha abolito le distanze. Per molte economie emergenti la tecnologia si è mostrata la chiave per attenuare il divario, ma altresì le politiche economiche mondiali non sono state immaginate a vantaggio dei più poveri ${ }^{19}$. Ł̀ evidente come la diminuzione del divario esistente dipenda in buona parte dall'introduzione e dall'implementazione degli strumenti tecnologici e dalla creazione di infrastrutture adeguate. La crescita dei redditi e dei prodotti lordi dipende in buona misura dalla diffusione della cultura, e internet in tal senso, se usato correttamente, si è rivelato uno strumento formidabile.

Vorrei concludere riassumendo un brano molto affascinante di uno dei principali maestri di Israele del secolo scorso, Rav Kook, che scrive ${ }^{20}$ che le anime più grandi non possono staccarsi dalla totalità più comprensiva. L'altezza di questa totalità è determinata solo dalla pienezza dei suoi individui e dei piccoli e grandi gruppi che la completano. Gli individui dotati dello spirito più alto sentono che gli individui necessitano di essere migliorati, che le migliori forme sociali devono essere innalzate. Desiderano che ogni individuo particolare sia preservato e sviluppato e che la collettività sia unita e in pace. In lui si fondono in ogni

17. J.B. Soloveitchick, La solitudine dell'uomo di fede, Livorno 2016, p. 21.

18. J. Sacks, La dignità cit., p. 39.

19. Ivi, p. 123.

20. Orot ha-qodesh 2, p. 442-445, citato in D. Linzer, Jewish Social Justice: A Universalist Mandate?, 2016, in https://ibrary.yctorah.org/2016/08/jewish-social-justice-a-universalist-mandate/. 
momento quattro canti, quello della propria anima, del proprio popolo, dell'umanità e del mondo. 
Religioni e sviluppo sostenibile
Vita umana, lavoro e sviluppo tecnologico

(obiettivi 3, 8 e 9 Agenda Onu 2030):

il punto di vista del cattolicesimo

con un'appendice dopo covid 19

Luca Peyron

Il cattolicesimo offre una propria posizione in rapporto ai tre temi di cui trattiamo trovando alimento e fondazione, come riguardo ad ogni tema del resto, in due capisaldi determinanti: la posizione della Scrittura e la Tradizione della Chiesa, tenuti insieme dalla teologia che offre al mondo un sapere fondato nella fede. In questo breve contributo tenterò di fornire una sintetica panoramica delle questioni in gioco, qualche concreto esempio e una piccola sintesi nell'ottica di costruire un bene che sia effettivamente comune. Per brevità, e onde evitare tecnicismi poco interessanti in questa sede, utilizzerò soprattutto i riferimenti alla Tradizione della Chiesa e al suo Magistero, ossia ai suoi pronunciamenti storicamente più programmatici e sistematici, rinvenendo in essi un'efficace sintesi anche della componente scritturistica.

È intuibile come in questo spazio non sia possibile una trattazione di temi così ampi ed importanti: dunque scelgo, di mettere in relazione i primi due temi del convegno, vita umana e lavoro, con il terzo, lo sviluppo tecnologico, evidenziando come i primi possano dare delle indicazioni preziose rispetto al governo del terzo e come inversamente 
il terzo, rispetto alla cultura e alla società che determina, abbia non poca incidenza sui primi due. In altri termini, da un lato possiamo partire da alcuni assunti, consolidati nella Tradizione e nel pensiero cattolico, per disegnare la tecnologia che vorremmo in termini antropologici, economici e di giustizia sociale; dall'altro, poiché non ci troviamo all'anno zero dello sviluppo tecnologico, è interessante evidenziare come questo abbia già significativamente modificato la percezione che l'essere umano ha di se stesso ed i conseguenti assetti e posture sociali che si sono determinati. Da quest'ultimo aspetto prenderemo quindi dapprima le mosse.

Tuttavia, un altro elemento va aggiunto: nella redazione di questo testo, successiva al convegno, abbiamo tutti vissuto la pandemia di Covid 19, che non ci può lasciare indifferenti in considerazione di quanto essa ha inciso nella carne del mondo e così significativamente nei suoi ordinamenti sociali, economici e religiosi. Dedicheremo, dunque, gli ultimi due paragrafi di questo contributo a provare a delineare alcuni possibili scenari futuri.

Lo sviluppo tecnologico e gli artefatti tecnologici in sé hanno una straordinaria capacità di produrre significati, paragonabile forse solo a quella del corpo. Essi conferiscono, infatti, significati nuovi alla realtà e rivelano con sorprendente tangibilità le posture culturali dei nostri contemporanei. In campo non cattolico lo aveva notato Marx, ad esempio, quando scriveva: «La tecnologia svela il comportamento attivo dell'uomo verso la natura, l'immediato processo di produzione della sua vita, e con essi anche l'immediato processo di produzione dei suoi rapporti sociali vitali e delle idee dell'intelletto che ne scaturiscono» ${ }^{1}$. Ma anche il grande interesse che il credente cattolico e la Chiesa hanno rispetto a questi temi è storicamente fondato, poiché sempre di più grazie agli strumenti tecnologici oggi disponibili vi è una forte ricaduta antropologica di ogni singola decisione e delle pratiche che via via si consolidano. Tale ricaduta è centrale per la fede e per la prassi pastorale della Chiesa chiamata ad essere segno di salvezza nel mondo e per il mondo, poiché l'essere umano è al centro dell'interesse di 
Vita umana, lavoro e sviluppo tecnologico

Dio, che lo ha creato a propria immagine e somiglianza. La cifra con cui si manifesta questo interesse, con cui la Chiesa ed i credenti giudicano ogni tempo e propongono alla società una visione della storia, della politica e dell'economia, è Cristo vero Dio e vero uomo. Sintetizzo questa posizione con alcune parole del proemio della Costituzione del Concilio Vaticano II Gaudium et Spes (in seguito GS), che illustra come la Chiesa voglia stare nel mondo contemporaneo:

Ai nostri giorni l'umanità, presa d'ammirazione per le proprie scoperte e la propria potenza, agita però spesso ansiose questioni sull'attuale evoluzione del mondo, sul posto e sul compito dell'uomo nell'universo, sul senso dei propri sforzi individuali e collettivi, e infine sul destino ultimo delle cose e degli uomini. Per questo il Concilio, testimoniando e proponendo la fede di tutto intero il popolo di Dio riunito dal Cristo, non potrebbe dare una dimostrazione più eloquente di solidarietà, di rispetto e d'amore verso l'intera famiglia umana, dentro la quale è inserito, che instaurando con questa un dialogo sui vari problemi sopra accennati, arrecando la luce che viene dal Vangelo, e mettendo a disposizione degli uomini le energie di salvezza che la Chiesa, sotto la guida dello Spirito Santo, riceve dal suo Fondatore. Si tratta di salvare l'uomo, si tratta di edificare l'umana società. (Proemio)

Già qualche anno prima però papa Pio XII invitava anche i singoli ministri ordinati ad occuparsi di questi temi: "Il sacerdote in cura d'anime può e deve sapere quel che affermano la scienza, l'arte e la tecnica moderna, in quanto riguardano il fine e la vita religiosa e morale dell'uomo» ${ }^{2}$.

Un'ulteriore considerazione degna di nota riguarda un tratto importante della tecnologia contemporanea, in particolare delle tecnologie emergenti, e lo desumiamo da una un'intuizione di Pierre Levy: «Una tecnica non è né buona né cattiva (dipende dagli usi, dai contesti e dai punti di vista) e neppure neutrale (essendo condizionante o cogente...). Non si tratta di valutarne gli impatti ma di individuare le irreversibilità in cui uno dei suoi usi ci immette, le occasioni che può permetterci di cogliere, e di formulare progetti che sfruttino le virtualità di cui è portatrice, decidendo cosa far-

2. Pio XII, Discorso sull'importanza della televisione, 21 ottobre 1955. 
ne in futuro» ${ }^{3}$. La tecnologia è irreversibile, difficilmente la società percorre a ritroso la via imboccata, - o se lo facesse o decidesse di farlo, troverebbe non poche resistenze nei consociati e certamente si dovrebbero pagare alti costi - . Dunque, prima di imboccare strade che avranno un rilevante impatto sulla nostra vita, è quanto mai necessario avere il più possibile contezza di quello che stiamo facendo e, soprattutto, avere la possibilità concreta di scegliere. Avverte Papa Francesco nell'enciclica Laudato Si (LS 105):

Si tende a credere che «ogni acquisto di potenza sia semplicemente progresso, accrescimento di sicurezza, di utilità, di benessere, di forza vitale, di pienezza di valori», come se la realtà, il bene e la verità sbocciassero spontaneamente dal potere stesso della tecnologia e dell'economia. Il fatto è che «l'uomo moderno non è stato educato al retto uso della potenza», perché l'immensa crescita tecnologica non è stata accompagnata da uno sviluppo dell'essere umano per quanto riguarda la responsabilità, i valori e la coscienza.

La possibilità di tale consapevolezza è, anzi, messa ulteriormente in crisi dall'attuale organizzazione economica e dalla diffusione delle tecnologie digitali: infatti buona parte di esse, quelle legate alle grandi imprese della Silicon Valley per capirci, hanno assunto posizioni di monopolio quasi permanenti e su molteplici fronti. Tuttavia va anche detto che tali posizioni di monopolio, se accettabili nei momenti iniziali della vita delle imprese, oggi sono difficilmente giustificabili: infatti, in base al principio di destinazione universale dei beni, secondo il quale «Dio ha destinato la terra e tutto quello che essa contiene all'uso di tutti gli uomini e di tutti i popoli, e pertanto i beni creati devono equamente essere partecipati a tutti, secondo la regola della giustizia, inseparabile dalla carità» (GS 69), la Chiesa ha una posizione chiara di condanna rispetto ai monopoli, in particolare quelli tecnologici.

«Le nuove conoscenze tecniche e scientifiche devono essere poste a servizio dei bisogni primari dell'uomo, affinché possa gradualmente accrescersi il patrimonio comune dell'umanità. La piena attuazione del principio della destinazione universale dei beni richiede, pertanto, azioni a 
Vita umana, lavoro e sviluppo tecnologico

livello internazionale e iniziative programmate da parte di tutti i Paesi: «Occorre rompere le barriere e i monopoli che lasciano tanti popoli ai margini dello sviluppo, assicurare a tutti - individui e Nazioni - le condizioni di base, che consentano di partecipare allo sviluppo» ${ }^{4}$.

Tali monopoli dunque non sono solo potenzialmente ingiusti per le ragioni immediatamente comprensibili legate al concetto stesso di monopolio: essi sono anche potenzialmente ingiusti ed antidemocratici nella misura in cui determinate tecnologie o applicazioni, come le piattaforme informatiche di cui tutti ci serviamo, non hanno alternative reali e praticabili. In altri termini, se tutti usiamo esclusivamente o quasi una determinata tecnologia, di cui una singola impresa è proprietaria, di fatto, anche se non di diritto, quell'impresa è proprietaria di un pezzo significativo della nostra vita (si pensi ad esempio alle piattaforme di messaggistica o a quelle utilizzate per le teleconferenze).

Il quadro di riferimento in cui il credente cattolico si muove, e dunque alla luce del quale queste e analoghe valutazioni sono fatte dalla Chiesa, resta comunque ed in definitiva quello escatologico, ossia la sua vocazione fontale e finale alla comunione con Dio nell'eternità. Tuttavia, la rivelazione di Dio avviene sempre in una «oeconomia salutis gestis verbisque»(Dei Verbum 2): ciò significa che la storicità non è semplicemente l'involucro esterno che riveste la verità sostanziale della rivelazione, bensì il luogo proprio dell'affermazione della verità di Dio, in quanto la realtà stessa della verità di Dio non si dà a prescindere dalla sua forma storica. Ciò, dunque, significa uno specifico interesse per le cose del mondo affinché possano essere segno e strumento di quelle eterne. In altri termini l'orizzonte ultimo dell'essere umano non è la storia intesa come succedersi delle vicende sociali e politiche, ma la verità stessa, fondante e insieme finale, della persona umana chiamata a rispondere a un invito di Dio ad entrare in relazione con Lui. Così GS 57:

I cristiani, in cammino verso la città celeste, devono ricercare e gustare le cose di lassù questo tuttavia non diminuisce, anzi aumenta l'importanza del loro dovere di collaborare

4. Cfr. Compendio della Dottrina Sociale della Chiesa n. 179 e passim Giovanni Paolo II, Centesimus annus, 35 
con tutti gli uomini per la costruzione di un mondo più umano. $\mathrm{E}$ in verità il mistero della fede cristiana offre loro eccellenti stimoli e aiuti per assolvere con maggiore impegno questo compito e specialmente per scoprire il pieno significato di quest'attività, mediante la quale la cultura umana acquista un posto importante nella vocazione integrale dell'uomo.

Tale interesse escatologico non comporta però semplicemente una critica alle attività umane, segnatamente alla tecnologia, quanto piuttosto e soprattutto, come si è evidenziato, un contributo positivo che nasce dal fatto che una visione escatologica dell'esistenza conferisce una visione teleologica della vita stessa. In altri termini la presenza di un giudizio sulla vita, che si attua nel momento della morte, conferisce un criterio operativo per orientare la vita a quei valori e verità che saranno oggetto del giudizio, i quali in positivo contribuiscono a formare l'esercizio positivo della libertà già nel tempo, anzi solamente ed eminentemente nel tempo. In termini scritturistici il lavoro manuale di Cristo durante la sua vita terrena è la radice e la cifra più alta ed immediatamente comprensibile di questi assunti uniti al suo sacrificio sulla croce.

È necessario, infine, fare una puntualizzazione rispetto all'espressione caratterizzante queste note, che è quella di: "sviluppo tecnologico". In questione non vi è la tecnica in quanto tale, ma la tecnologia ed il suo sviluppo: non si tratta di fare giochi di parole, ma tecnica e tecnologia non sono il medesimo concetto e non delimitano il medesimo perimetro. Mentre la tecnica è semplicemente l'insieme delle attitudini operative in un determinato settore, la tecnologia è anche una riflessione culturale su di essa. Ma oggi è certo che l'artefatto tecnico ha ormai una portata culturale persino preponderante rispetto all'artefatto in sé e per sé. Tuttavia, è chiaro che se due lessemi diversi sono compresi come sinonimi, anzi aver sostituito e oggi spesso il primo è usato in modo interscambiabile con il secondo, non è un passaggio di poco conto: dato che, come ci rammenta la linguistica, il rapporto significato, significante e referente extralinguistico è unico e irripetibile singolarmente solo per ogni parola, quando questo si rompe è avvenuto in società un passaggio degno di nota. Chiarito che il problema consiste quindi nell'interpretazione del valore e del significato da 
Vita umana, lavoro e sviluppo tecnologico dare alle novità tecnologiche, la lettura cattolica della tecnologia si pone come alternativa rispetto alle due letture che perlopiù vengono adottate. La prima presenta una visione sostanzialmente deterministica della tecnologia secondo la quale, in buona sostanza, essa è controllo razionale della natura, svincolata però dalla società e che ha solo successivamente un effetto trainante nel mutamento della società stessa $^{5}$. Dall'altra si pone la lettura costruttivista secondo cui le tecnologie non hanno successo esclusivamente su base funzionale: infatti, per ogni problema tecnico esiste una varietà di possibili soluzioni, alternative ma spesso identiche, per cui il successo storico di una rispetto ad un'altra dimostra che altri sono i fattori determinanti. L'esempio tipico, portato da uno dei maggiori rappresentanti di questa posizione, Feenberg, è quello della bicicletta. Tra bicicletta con ruote di diametro diverso e con ruote di diametro uguale ha vinto la seconda perché si è preferito una maggiore stabilità rispetto ad una maggiore velocità che la prima avrebbe dato. Scelta non tecnica, egli sottolinea, ma sociale ${ }^{6}$. La terza via, quella della Chiesa e della Dottrina Sociale della Chiesa, conserva gli aspetti giudicati positivi delle due visioni, ma prende le mosse da diversi presupposti e soprattutto ha come fine quello di far emergere il carattere rivelativo che la tecnologia può avere del senso che precede la stessa natura umana, il suo fondamento di libertà e responsabilità ${ }^{7}$. Rileva Benedetto XVI:

La tecnica attrae fortemente l'uomo, perché lo sottrae alle limitazioni fisiche e ne allarga l'orizzonte. Ma la libertà umana è propriamente se stessa solo quando risponde al fascino della tecnica con decisioni che siano frutto di re-

5. Di fatto il precursore di questa corrente è in qualche modo Marshall McLuhan che, ad esempio in La galassia Gütenberg, Armando Editore, 1976, sottolinea l'importanza dei media nella storia umana rilevando l'influenza della stampa a caratteri mobili sulla storia della cultura occidentale. La stampa non è stata inventata per risolvere un problema sociale ma tecnico, la sua applicazione ha però poi sconvolto in maniera radicale diversi ordini sociali. Sulla questione, in particolare rispetto ai primordi della rivoluzione digitale, cfr. P. Aroldi, B. Scifo, Internet e l'esperienza religiosa in rete, Vita e Pensiero, Milano 2002. Per una lettura fortemente critica dell'approccio determinista e delle sue conseguenze sulla società in modo significativo cfr. J. Ellul, La tecnica. Rischio del secolo, Giuffré, Milano 1969 e Il sistema tecnico, Editoriale Jaca Book, Milano 2009.

6. A. Feenberg, Critical Theory of Technology, Oxford University Press, Oxford 1991.

7. Per una ricognizione sintetica di autore e posizioni su questi temi cfr. P. Benanti, Tecnologia e sviluppo umano nella Caritas in veritate, «Il Regno Documenti», 1(2014), pp. 50-64. 
sponsabilità morale. Di qui, l'urgenza di una formazione alla responsabilità etica nell'uso della tecnica. A partire dal fascino che la tecnica esercita sull'essere umano, si deve recuperare il senso vero della libertà, che non consiste nell'ebbrezza di una totale autonomia, ma nella risposta all'appello dell'essere, a cominciare dall'essere che siamo noi stessi. ${ }^{8}$

\section{Lo sguardo della Tradizione del Magistero}

Il legame tra lavoro e vita umana è ben presente nella riflessione e nella preghiera della Chiesa cosicché al lavoro è dedicato un intero capitolo del Compendio della Dottrina Sociale $^{9}$ vedi sopra che è un testo che riassume le posizioni della Tradizione maturate sino ad oggi. Riporto alcuni passaggi centrali per i temi qui in discussione segnalando alcuni termini con l'aggiunta di grassetti. Al numero 255 si legge:

L'Antico Testamento presenta Dio come Creatore onnipotente (cfr. Gen 2,2; Gb 38-41; Sal 104; Sal 147), che plasma l'uomo a Sua immagine, lo invita a lavorare la terra (cfr. Gen 2,5-6) e a custodire il giardino dell'Eden in cui lo ha posto (cfr. Gen 2,15). Alla prima coppia umana Dio affida il compito di soggiogare la terra e di dominare su ogni essere vivente (cfr. Gen 1,28). Il dominio dell'uomo sugli altri esseri viventi, tuttavia, non deve essere dispotico e dissennato; al contrario, egli deve «coltivare e custodire» (cfr. Gen 2,15) i beni creati da Dio: beni che l'uomo non ha creato, ma ha ricevuto come un dono prezioso posto dal Creatore sotto la sua responsabilità. [...] Nel disegno del Creatore, le realtà create, buone in se stesse, esistono in funzione dell'uomo. Lo stupore davanti al mistero della grandezza dell'uomo fa esclamare il salmista: «Che cosa è l'uomo perché te ne ricordi e il figlio dell'uomo perché te ne curi? Eppure l'hai fatto poco meno degli angeli, di gloria e di onore lo hai coronato: gli hai dato potere sulle opere delle tue mani, tutto hai posto sotto i suoi piedi. ( Sal 8,5-7)

L'iniziativa è sempre di Dio: è Lui che plasma l'uomo, è Lui che crea il mondo ed è sempre Lui che lo dona all'essere umano. Tale opera non si ferma, però, ad un momento fontale: Il dono determina il nascere di una relazione che

8. Benedetto XI, Caritas in Veritate, 70 .

9. Pontificio Consiglio della Giustizia e della Pace, Compendio della Dottrina Sociale della Chiesa, Libreria Editrice Vaticana, Roma 2004. 
Vita umana, lavoro e sviluppo tecnologico

ha i tratti di un'alleanza concreta e stabile di cui Dio stesso si fa garante mediante promesse irrevocabili. Tale antecedenza e alleanza sono così garanzia che l'opera dell'uomo non sia un'opera solitaria, che altrimenti sarebbe titanica ed impossibile; nello stesso tempo, proprio perché alleanza, l'antecedenza ha la possibilità, se accolta nella libertà ed accolta nella responsabilità del contraente umano, di custodire i doni da un uso improprio. Come conseguenza di queste premesse, La stessa tecnica è in qualche modo dono di Dio come sottolinea Pio XII nell'enciclica Miranda Prorsus del 1957: «Le meravigliose invenzioni tecniche, di cui si gloriano i nostri tempi, benché frutti dell'ingegno e del lavoro umano, sono tuttavia doni di Dio, nostro creatore, dal quale proviene ogni opera buona».

La salvezza viene da Dio, non è opera dell'uomo né come singolo né come società e, dunque, neppure e tanto meno dalla tecnologia e dai suoi derivati: questo mette al riparo da un sempre possibile delirio di onnipotenza - che storicamente ha portato l'umanità a rischiare più volte di distruggere se stessa e certamente ha già avuto come esito quello di distruggerne una parte -. Il sociologo Luciano Gallino aveva messo in guardia sul potenziale dannoso della hybris creativa dell'uomo tecnologico: «Nell'inventare e nel costruire entità artificiali, quali sono le macchine, nel manipolare per loro tramite noi stessi e il mondo, la tecnologia ci consente di dar corpo, col vantaggio di non obbligarci a confessarlo, al nostro desiderio segreto di riprodurre, a nostra volta, l'avventura creatrice di Dio» ${ }^{10}$.

Linvito di Dio a plasmare la terra rappresenta per converso l'autorizzazione a servirsi in pienezza delle facoltà che gli sono state conferite e che hanno come scopo ultimo quello di lodare Dio, cioè di entrare in una relazione amorosa con Lui che altro non è, in ultima istanza, che la beatitudine promessa al di là nella morte nell'eternità. La funzione delle realtà create, dunque, e il lavoro che l'essere umano a cui le sottopone hanno lo scopo di orientare la persona in questa salvifica direzione.

In questo quadro non si deve tacere la dimensione del peccato dell'essere umano, ossia la dimensione del male che 
inficia un corretto uso della libertà e della responsabilità. Nella narrazione biblica è interessante notare come questa componente primordiale sia collegata alla tecnica rivelandone la non neutralità: rinveniamo infatti questo accostamento laddove l'autore sacro descrive la vicenda e la figura di Caino, il progenitore omicida del fratello Abele. Caino e Abele incarnano non solo opposti come bene e male, ma anche complementari come lo sono pastorizia e agricoltura, nomadismo e vita stanziale. Di fatto il protagonista del racconto è Caino solamente, mentre Abele resta sullo sfondo, egli è la parola di bene che non può più essere detta perché soffocata dal male, la possibilità di vita buona che il peccato spegne; Caino però non è solo il male e lo dimostra il fatto che Dio metta un segno su di lui affinché nessuno lo tocchi, affinché possa avere ed abbia una discendenza. Caino è all'origine di tecnica ed arte (cfr. Gen 4-5) e questo suo carattere, unito al lavoro, è la modalità di redenzione ed espiazione che Dio assegna all'essere umano per purificarsi del male di cui è capace. Il racconto archetipo della Bibbia ci trasmette l'idea concretissima che il lavoro è forma di redenzione e conferisce una particolare dignità all'uomo che con esso può rimediare ai danni causati dal male che ha accettato di compiere. In questo esercizio la dimensione tecnica ed artistica sono un'espressione eminente e insieme ambivalente: è dunque la tecnologia, la riflessione sulla tecnica, il campo dove si gioca questa partita determinante, con la consapevolezza che vi è in essa una radice potenziale di male che può essere continuamente risvegliata dalla libertà umana. Tale ambivalenza come può essere affrontata e risolta? Principalmente questo è il tema in cui desideriamo ora addentrarci.

\section{Potere, servizio e bene comune}

Esaustivo e significativo papa Paolo VI nella Populorum Progressio (in seguito $\mathrm{PP}$ ):

La tecnocrazia di domani può essere fonte di mali non meno temibili che il liberalismo di ieri. Economia e tecnica non hanno senso che in rapporto all'uomo ch'esse devono servire. E l'uomo non è veramente uomo che nella misura in cui, padrone delle proprie azioni e giudice del loro valore, diventa egli stesso autore del proprio progresso, in conformità con la natura che gli ha dato il suo Creatore e 
Vita umana, lavoro e sviluppo tecnologico di cui egli assume liberamente le possibilità e le esigenze, (PP 34)

a cui fa eco Giovanni Paolo II nella sua enciclica programmatica, la Redemptor Hominis (RH), in cui afferma:

Esiste già un reale e percettibile pericolo che, mentre progredisce enormemente il dominio da parte dell'uomo sul mondo delle cose, di questo suo dominio egli perda i fili essenziali, e in vari modi la sua umanità sia sottomessa a quel mondo, ed egli stesso divenga oggetto di multiforme, anche se spesso non direttamente percettibile, manipolazione, mediante tutta l'organizzazione della vita comunitaria, mediante il sistema di produzione, mediante la pressione dei mezzi di comunicazione sociale. (RH 16)

Decisivo e implicito in questi passaggi il concetto di antropocentrismo, che contiene però - come nella vita di Caino - delle ambivalenze: l'antropocentrismo tout court è infatti stato sottoposto a dura critica soprattutto in relazione a questioni di etica ambientale ed un antropocentrismo forte, che riconosce diritti esclusivamente all'essere umano, è una posizione oggi difficilmente sostenibile. Esso non significa un semplice e generico mettere l'uomo al centro, ma implica nella dottrina cristiana lo sviluppo di questo concetto. Come declinare allora, in scelte ed architetture, la centralità dell'umano? Credo particolarmente interessante accostare a questo auspicio due concetti: quello di gratuità/generosità e quello di bene comune.

Abbiamo accennato in precedenza come a monte dell'esistenza umana e del rapporto con Dio, del lavoro e della stessa tecnica, ci sia un dono, il donare libero di Dio creatore. La categoria del dono e delle generosità, come ha notato Benedetto XVI nella Caritas in Veritate (CV) sono essenziali: «La gratuità è presente nella sua vita in molteplici forme, spesso non riconosciute a causa di una visione solo produttivistica e utilitaristica dell'esistenza. L'essere umano è fatto per il dono, che ne esprime ed attua la dimensione di trascendenza»(CV 34). Il Pontefice ha dichiarato in tal mondo la necessità che vi sia un dominio dello spirito sulla materia e che questo si manifesti proprio con il dono. La gratuità e la generosità rappresentano infatti un calmiere straordinario a due diverse tensioni negative: l'individualismo radicato nell'essere umano peccatore e il conformismo 

cui portiamo attenzione è l'atteggiamento secondo cui si è di fatto contrari all'unione sociale, condannato chiaramente. Nel magistero della Chiesa ${ }^{11}$ si esplica in un agire sociale ispirato ai principi di sussidiarietà e solidarietà. L'individualismo è considerato una piaga perché diventa autoreferenzialità annullando qualsiasi previsione etica e negando la stessa struttura relazionale dell'essere persona, che proprio nel lavoro si manifesta in modo eminente. Così Benedetto XVI:

Sebbene la difesa dei diritti abbia fatto grandi progressi nel nostro tempo, la cultura odierna, caratterizzata, tra l'altro, da un individualismo utilitarista e un economicismo tecnocratico, tende a svalutare la persona. Questa viene concepita come un essere «fluido», senza consistenza permanente. Nonostante sia immerso in una rete infinita di relazioni e di comunicazioni, l'uomo di oggi paradossalmente appare spesso un essere isolato, perché indifferente rispetto al rapporto costitutivo del suo essere, che è la radice di tutti gli altri rapporti, quello con Dio. L'uomo d'oggi è considerato in chiave prevalentemente biologica o come «capitale umano», «risorsa», parte di un ingranaggio produttivo e finanziario che lo sovrasta. Se, da una parte, si continua a proclamare la dignità della persona, dall'altra, nuove ideologie [...] contribuiscono a considerare il lavoratore dipendente e il suo lavoro come beni «minori» e a minare i fondamenti naturali della società, specialmente la famiglia. In realtà, l'essere umano, costitutivamente trascendente rispetto agli altri esseri e beni terreni, gode di un reale primato che lo pone come responsabile di se stesso e del creato. Concretamente, per il Cristianesimo, il lavoro è un bene fondamentale per l'uomo, in vista della sua personalizzazione, della sua socializzazione, della formazione di una famiglia, dell'apporto al bene comune e alla pace. Proprio per questo, l'obiettivo dell'accesso al lavoro per tutti è sempre prioritario, anche nei periodi di recessione economica. ${ }^{12}$

Interessante notare che nella Scrittura l'individualismo si esprime esemplarmente proprio attraverso la tecnica, rom-

11. «Piacque a Dio di santificare e salvare gli uomini non individualmente e senza alcun legame tra loro, ma volle costituire di loro un popolo» (Lumen Gentium 9)

12. Benedetto XVI, Discorso del santo padre Benedetto XVI ai partecipanti alla plenaria del pontificio consiglio della giustizia e della pace, 3 dicembre 2012. 
Vita umana, lavoro e sviluppo tecnologico

pendo per sempre la comunione umana. Si tratta dell'episodio di Babele ove la hybris prometeica dell'uomo costruttore e facitore di se stesso lo porta a confliggere e rompere quell'unità fondativa rappresentata dalla lingua comune. Nel Nuovo Testamento Gesù afferma con chiarezza: «Questa è la vita eterna: che conoscano te, l'unico vero Dio, e colui che hai mandato, Gesù Cristo» (Gv 17,3). La vita autentica è fatta di relazioni così come la stessa vita intradivina è strutturata e si è rivelata come relazione di persone, non di individui.

La seconda tensione che esaminiamo è il "conformismo computazionale" della cultura digitale ${ }^{13}$. La cultura tecnologica e digitale deriva buona parte della sua struttura epistemologica dalla struttura della macchina: essa ha infatti per obiettivo funzionare ed in modo il più performante possibile. Affinché una macchina funzioni e funzioni bene, però, è necessario che tutti i pezzi siano al loro posto e ben connessi tra loro. È chiaro che più le strutture sono omologhe ed omologabili più diventano funzionali al funzionare. É questa la struttura che chiamo conformismo computazionale: gli assetti sociali, imprenditoriali e tecnici che hanno bisogno di omologazione per potersi relazionare, poter utilizzare e poter ricevere vantaggio dalla cultura digitale, ma essa comporta appiattimento delle differenze, impoverimento della molteplicità e, di fatto, sterilizzazione della vita intesa come capacità creativa latu sensu. Questa tendenza l'aveva già individuata e stigmatizzata in tempi non sospetti lo storico francese Jacques Ellul, il quale nel suo Il bluff tecnologico scrive:

Il primo paradigma di questa assurdità è la volontà di standardizzare tutti. È una tendenza antica, rimasta tale fino ad oggi. Nella società tecnologica si cerca di creare standard per ogni cosa allo scopo di poter poi applicare tecniche di universalizzazione e modellazione. A questo scopo si standardizza il linguaggio (serve al computer per funzionare meglio), l'idoneità al lavoro, la formazione scolastica, i prodotti, ecc. La standardizzazione globale che agisce attraverso i media e i prodotti tecnologici e informatici, non è più un semplice prodotto della rivoluzione industriale (il 
Papa Francesco stigmatizza questa tendenza: «Sul piano personale, l'epoca digitale cambia la percezione dello spazio, del tempo e del corpo. Infonde un senso di espansione di sé che sembra non incontrare più limiti e l'omologazione si afferma come criterio prevalente di aggregazione: riconoscere e apprezzare la differenza diventa sempre più difficile» ${ }^{15}$.

La gratuità/generosità ha la capacità di rompere questi schemi minandoli al loro interno. La generosità è per sua definizione a-computabile, o non computabile: essa è sempre altro ed oltre quello che numericamente può essere definito o progettato. La generosità che genera gratuità fa sì che sporgersi verso l'altro sia un moto del cuore e mai una scelta funzionale a qualche tornaconto. Si tratta di una categoria poco frequentata nella Scrittura ma è certamente riconducibile all'amore; usiamo e preferiamo il concetto di generosità e quello di gratuità perché amore rischia di essere un termine ormai ambiguo, consunto da diversi usi impropri e talora ideologici che ne hanno diluito la significanza ${ }^{16}$.

Venendo al terzo concetto centrale di questo nostro discorso, il concetto di "bene comune" è espressione validata nelle carte costituzionali e nei grandi trattati internazionali, ma fa fatica ad essere declinato in specifico. Per alcuni il bene comune è un concetto ormai impraticabile in un mondo fortemente individuale e multiculturale poiché sarebbe di fatto impossibile in un processo democratico un minimo comun denominatore valoriale. Per la Dottrina sociale della Chiesa per bene comune possiamo intendere: «Linsieme di quelle condizioni della vita sociale che permettono sia alle collettività sia ai singoli membri, di raggiungere la propria

14. J. Ellul, Il bluff tecnologico citato in C. Mazzucchelli, Nei labirinti della tecnologia, Delos Digital, 2014, ebook.

15. Francesco, Discorso alla plenaria della Pontificia accademia per la vita, 28 febbraio 2020.

16. Sempre più spesso, per non dire sempre, è necessario accompagnare il termine amore da aggettivi quali vero, autentico, puro, disinteressato etc. L'esigenza di aggettivare un termine dimostra in qualche modo che tale termine non ha più la forza di vivere di vita propria, di essere esaustivo in se stesso. Sul punto mi permetto di rimandare nuovamente a L. Peyron, I. Andreis, Elogio della generosità, Elledici, Torino 2019. 
Vita umana, lavoro e sviluppo tecnologico

perfezione più pienamente e più celermente ${ }^{17}$. Tale definizione dunque non consiste nella definizione di un insieme di valori, quanto piuttosto di una pre-condizione, per così dire, di umanizzazione. In altri termini il bene comune prima di tutto è ciò che permette all'essere umano di scoprirsi tale e diventare sempre più tale. Introdurre nella questione della centralità dell'umano il bene comune significa allora recuperare la dimensione di prossimità e di relazionalità propriamente e tipicamente umana, propria della natura umana al di là di ogni forma culturale, politica o religiosa; una dimensione che la semplice efficienza e connessione computazionale non possono considerare. Bene comune significa, prima di tutto, quindi non l'adesione ad un quadro etico o di interessi diversi condivisi che permettano la sussistenza di rapporti sociali, ma piuttosto una cornice in cui l'agire diventa una determinazione di sé, dell'indole relazionale e personalistica dell'essere umano: dunque il bene comune non è prima di tutto un corpus in qualche modo negoziato tra singolo e molteplicità o tra tradizioni culturali diverse chiamate ad insistere nello stesso tempo e nello stesso luogo; esso si configura piuttosto come un appello all'intima radice personale e libertaria dell'individuo. In questa prospettiva la prossimità non è una scelta o una opzione quanto piuttosto un carattere strutturale dell'essere umano che va riconosciuto ed accolto. Il fatto che l'altro ci sia, lo possa vedere e comprendere mi restituisce e conferisce una dimensione personale, un io finalmente riconoscibile da un tu ed un noi in forza del quale dare senso al mio io altrimenti isolato ed incapace di generare. In questa prospettiva il prossimo non è una potenziale minaccia, ma una risorsa indispensabile, un bene che sussiste solo perché riconosciuto come comune. In questa prospettiva, che non possiamo approfondire in tutte le sue conseguenze, il bene comune nella cultura digitale assume una nuova e più densa significanza. Se la società digitale assume come fine il bene comune, tale fine ne giudica continuamente i mezzi e custodisce la centralità dell'essere umano entro i termini in cui egli riconosce ed agisce per riconoscere l'essere umano

17. Cfr. Concilio Vaticano II, Gaudium et spes, 26; cfr. Catechismo della Chiesa Cattolica, 1905- 1912; Giovanni XXIII, Mater et magistra; Id., Pacem in terris; Paolo VI, Octogesima adveniens, 46. 
altro da sé e con esso il mondo che lo circonda che diventa strumento necessario a tale custodia. Nella cultura digitale non può esistere un generico essere umano al centro, esiste il mio prossimo che posso amare o odiare. Nella cultura digitale io posso creare un'architettura di senso, di strumenti, di regole e di prassi che mi permettano di amare il prossimo, possibilmente come me stesso, così da poter esserne riamato.

Come si accennava all'inizio, però, l'irrompere della pandemia di Covid-19 ci impone di affinare lo sguardo e provare a fare esercizio di profezia individuando alcuni scenari e linee di pensiero, che possono essere di utilità comune soprattutto in previsione di quanto avverrà.

4. Il mondo dopo covid 19: tecnologia, distanza sociale e lavoro Questo paragrafo non può che soffrire di recentismo, poiché nasce nelle more della pandemia che ha sconvolto l'intero pianeta ed il nostro Paese nei primi mesi del 2020. Un articolo a firma di Gordon Lichfield, l'editore capo del MIT Technology Review, apparso il 17 marzo e dal perentorio titolo We're not going back to normal ${ }^{18}$ ha dato il via a livello planetario ad una serie piuttosto disordinata di commenti di ogni tipo, tutti sotto il segno del nulla sarà come prima. La tesi di Lichfield, basata su alcuni studi dell'Imperial College di Londra, è che questa non è altro che la pandemia che ci avverte che altre ne seguiranno, in una catena continua che ci porterà ad abituarci a delle posture sociali, politiche ed economiche del tutto inedite. Questo quadro, in effetti piuttosto verosimile, ci riporta agli obbiettivi dell'Agenda 2030 della Nazioni Unite da cui ha preso le mosse il convegno. Lobiettivo 3 recita: «Assicurare la salute e il benessere per tutti e per tutte le età», l'obiettivo 8: «Incentivare una crescita economica duratura, inclusiva e sostenibile, un'occupazione piena e produttiva ed un lavoro dignitoso per tutti», e infine l'obiettivo 9: "Costruire un'infrastruttura resiliente e promuovere l'innovazione ed una industrializzazione equa, responsabile e sostenibile». La pandemia ha ampiamente dimostrato come questi siano obiettivi perché 
Vita umana, lavoro e sviluppo tecnologico

la nostra attuale condizione se ne discosta significativamente, obiettivi di lungo o lunghissimo periodo che solo un uso diffuso, partecipato, inclusivo ed orientato a principi di sussidiarietà, solidarietà e del bene comune della tecnologia può rendere raggiungibili.

Durante la pandemia si è fatto un uso massivo di tecnologia, non solo immediatamente legata alle questioni sanitarie, ma più in generale per il tracciamento della diffusione del morbo e, nella ferialità della vita dei consociati, per mantenere il più possibile operativi alcuni settori e la coesione sociale. Alcuni fenomeni hanno trovato in tempo di crisi particolare terreno fertile come la diffusione delle fake news al punto da coniare il neologismo infodemia ${ }^{19}$. Però, rispetto ai temi trattati in questo breve studio vi è una questione che merita particolare attenzione: la gestione del nostro spazio sociale e delle modalità di lavoro attraverso tecnologie digitali. Di fatto Covid 19 ha realizzato quanto Mark Dery scriveva del 1996: «La velocità di fuga è la velocità richiesta a un corpo, per esempio un'astronave, per vincere la forza d'attrazione di un altro corpo, per esempio la Terra. Giorno dopo giorno, sembra essere più vicino il momento in cui la cultura informatica, la cosiddetta cybercultura, sia sul punto di raggiungere questa velocità di fuga $»^{20}$. Un virus, che ha il nome di una astronave della flotta stellare, ci ha violentemente portato in uno spazio che per molti anni abbiamo descritto in molti modi, più o meno distopici, più o meno apocalittici, in cui però ora stiamo per essere o siamo già. E uno spazio che mischia noto ed ignoto, uno spazio fatto di tecnologia, distanza, esperimenti che diventano prassi, prassi inveterate che diventano improvvisamente vetuste. Tra le novità più rilevanti per il loro impatto sulla vita individuale, c’è il fatto che durante la pandemia la tecnologia digitale ha facilitato il lavoro con lo smart working, i processi educativi con la teledidattica e la socialità delle persone veicolata da piattaforme social e di messaggistica di varia natura. La pervasività dello strumento digitale, unitamente

19. Per infodemia, secondo l'Enciclopedia Treccani, si intende la circolazione di una quantità eccessiva di informazioni, talvolta non vagliate con accuratezza, che rendono difficile orientarsi su un determinato argomento per la difficoltà di individuare fonti affidabili.

20. M. Dery, Velocità di fuga. Cyberculture a fine millennio, Feltrinelli, Milano 1997, p. 9. 
alla situazione emergenziale, ha rapidamente reso usuale quanto era eccezionale, abituandoci velocemente a modalità nei confronti delle quali la società aveva significative resistenze. È importante sottolineare che la digitalizzazione dei rapporti sociali in questo periodo non è stata per tutti uguale e l'accesso a tali servizi ha per molti versi acuito distanze e sperequazioni sociali. Un esempio tra molti: l'impossibilità per molte famiglie di avere connessione bastevole per far studiare i propri figli on line, così come la mancanza di devices sufficienti per tutti. Al netto di tali considerazioni è del tutto evidente che quanto è avvenuto ha segnato nel profondo i singoli e la società. Non è la prima pandemia della storia e non è la prima volta che una epidemia ha stravolto i rapporti umani: nel corso del tempo, che fosse peste o colera, l'altro ci è già sembrato un potenziale nemico, un untore; sconosciuto o parente l'abbiamo visto con sospetto e il darsi la mano un gesto eversivo. Nonostante quei terribili giorni, in cui la popolazione mondiale è stata drammaticamente falcidiata, siamo più o meno lentamente tornati ad una relazionalità che sino a qualche mese fa avremmo chiamato ordinaria. Semplificando molto, siamo andati oltre le precedenti epidemie a motivo della nostra struttura sostanzialmente relazionale. In altri termini abbiamo messo da parte la paura dell'altro perché dell'altro abbiamo fisicamente bisogno per non impazzire di solitudine, per perpetuare la vita, per essere vivi giorno per giorno. La grande novità storica, raggiunta la velocità di fuga, è che nell'immediato domani, rispetto all'altro ieri, avremo il digitale, la sua cultura ed i suoi strumenti. In questo tempo di pandemia abbiamo ampiamente supplito alla corporeità con la sua rappresentazione digitale. Limmateriale racconto di noi ha interfacciato in maniera impeccabile con gli altri, permettendoci un livello di socialità non indifferente, salvifico. I contatti fisici indifferibili li abbiamo schermati con mascherine e tute, tutti gli altri li abbiamo schermati con dei dispositivi. Quando il distanziamento sociale non è più necessario, ma la paura dell'altro rimane dentro, quale atteggiamento riscontiamo nelle persone? Come si è avuto modo di ripetere a più riprese la tecnologia ha per sua natura un carattere fortemente pervasivo e facilitandoci la vita genera processi difficilmente reversibili. Se oltre alla facilità del mezzo aggiungiamo la componente della paura, 
Vita umana, lavoro e sviluppo tecnologico

come saranno vissute le iterazioni interpersonali nel nuovo spazio in cui Covid 19 ci ha portato tutti? Fare esercizio di futurologia non è serio né consono, ma la posizione credente permette invece di fare un più affidabile esercizio di profezia ${ }^{21}$. Anziché attendere il futuro che verrà, abbandonandomi ad un fatalismo tecnologico, cioè lasciando che sia la tecnologia a disegnarlo, vorrei poter mettere parola, anzi gettare oggi un seme per terraformare questo nuovo spazio che andremo ad abitare. Penso sia importante farlo adesso, farlo subito, per poterci rendere conto che è cosa possibile nelle ristrettezze in cui siamo, e quindi ancora più promettente nel momento in cui avremo riacquisito la libertà fondamentale di abbracciare uno sconosciuto per strada. Come farlo? Non posso che tratteggiare delle suggestioni, ma comincerei con il mettere sin da subito in campo tutto ciò che non è computazionale, digitale, dinsintermediato, ma piuttosto corporeo ed autenticamente umano. Abbiamo bisogno da subito di un surplus di atti autenticamente umani ed abbiamo un serbatoio efficace ove reperirli: il Vangelo. Il Vangelo è pieno di atti umani, autenticamente umani, sono gli atti divini, autenticamente divini di Gesù . Non è un gioco di parole: per un credente Cristo è vero Dio e vero uomo, dunque ogni suo gesto, parola, invito è partecipato tanto dalla natura umana quanto da quella divina. Gli atti umani di Cristo sono quanto di meglio Dio ci offre e per un non credente o diversamente credente, quanto di meglio l'esperienza umana ci offre. Come rinvenire tali atti, come nel contesto odierno scoprire la verità e la validità di tali atti? È la Scrittura stessa a darcene indicazione, come il Magistero ha sottolineato: attraverso i poveri ed i piccoli.

Scrive Papa Francesco ai giovani nel 2014:

I poveri non sono soltanto persone alle quali possiamo dare qualcosa. Anche loro hanno tanto da offrirci, da insegnarci. Abbiamo tanto da imparare dalla saggezza dei poveri! [...] Ci insegnano che una persona non vale per quanto possiede, per quanto ha sul conto in banca. Un povero, una persona priva di beni materiali, conserva sempre la

21. La differenza sta nel fatto che la futurologia immagina gli scenari futuri scegliendo di non stare nelle regole grazie alla potenza della narrazione; la profezia invece è coltivazione della speranza, cioè di un base solida che ci sta alle spalle, per guardare il presente tanto in profondità da poter con esso generare un futuro desiderabile. 
sua dignità. I poveri possono insegnarci tanto anche sull'umiltà e la fiducia in Dio. Nella parabola del fariseo e del pubblicano (Lc 18,9-14), Gesù presenta quest'ultimo come modello perché è umile e si riconosce peccatore. Anche la vedova che getta due piccole monete nel tesoro del tempio è esempio della generosità di chi, anche avendo poco o nulla, dona tutto (Lc 21,1-4).22

Anche i piccoli, i bambini, possono essere maestri di saggezza, non vincolati dalle nostre paure ed insicurezze. Come ha dimostrato la psicologa Alison Gopnik: «La mia tesi è che l'infanzia è ciò che ci rende umani» ${ }^{23}$, i bambini possono essere la nostra riserva di futuro, non semplicemente biologico, ma anche morale ed esistenziale. Ella dimostra come: «I bambini piccoli risultano di fatto più intelligenti, fantasiosi e premurosi, e persino più consapevoli, di quanto non lo siano gli adulti» ${ }^{24}$ confermando in qualche modo le parole della Scrittura, segnatamente il salmo 8 che recita: «O Signore, nostro Dio, quanto è grande il tuo nome su tutta la terra: sopra i cieli si innalza la tua magnificenza. Con la bocca dei bimbi e dei lattanti affermi la tua potenza contro i tuoi avversari, per ridurre al silenzio nemici e ribelli». Il salmo che papa Paolo VI scelse per essere inviato come messaggio personale sulla Luna nello sbarco del 1969.

Gesù ha detto che i poveri li avremmo avuti sempre tra noi: i poveri ci salveranno dalle nostre prigioni digitali in cui, passato tutto, continueremo a chiuderci, entusiasti che tutto si possa disintermediare tenendoci al riparo dalle nostre paure. I poveri ci ricorderanno tutta la materialità di cui siamo felicemente fatti, i poveri ci aiuteranno a fare atti umani che ci umanizzeranno e ci aiuteranno a restare umani in un mondo dove le macchine saranno a servizio di questo unico vero, straordinario fine: l'essere umano, gli esseri umani, abitanti di un unico fragile pianeta. I poveri ed i piccoli, i bambini, coloro che hanno sempre il cuore ed il desiderio di vita più sviluppato di prudenza e scetticismo. La Scrittura ha espressioni efficaci che possono riassumere

22. Francesco, messaggio del Santo Padre Francesco per la XXIX Giornata mondiale della Gioventù 2014.

23. A. Gopnik, Il bambino filosofo. Come i bambini ci insegnano a dire la verità, amare e capire il senso della vita, Bollati Boringhieri, Torino 2010, p. 16.

24. Ivi, p. 17. 
Vita umana, lavoro e sviluppo tecnologico quanto si è venuti dicendo così: dalla bocca dei lattanti verrà la saggezza, dalle piaghe dei poveri avremo speranza.

\section{Conclusioni}

Sintetizzo quanto sono venuto argomentando rimescolando le parole del titolo del convegno con questo esito: lavoro e tecnologia per uno sviluppo umano della vita. Citando Paolo VI:

Lo sviluppo non si riduce alla semplice crescita economica. Per essere autentico sviluppo, deve essere integrale, il che vuol dire volto alla promozione di ogni uomo e di tutto l'uomo. Com'è stato giustamente sottolineato da un eminente esperto: "noi non accettiamo di separare l'economico dall'umano, lo sviluppo dalla civiltà dove si inserisce. Ciò che conta per noi è l'uomo, ogni uomo, ogni gruppo d'uomini, fino a comprendere l'umanità intera. (PP 14)

Ogni essere umano è alla ricerca del proprio vero volto e compito nella storia ed ognuno dunque percepisce l'enigma inevitabile di dover decidere di sé. Il lavoro e la tecnologia, nella visione e nella percezione cattolica, debbono essere prima che a servizio del fare a servizio dell'essere, dell'essere e divenire autenticamente umani. Il sapere ed il sapere fare si configurano come strumenti a servizio della scoperta dell'essere e delle scelte di libertà e responsabilità che portano a sapere essere. La vocazione cristiana come chiamata di Dio ad una vita piena consiste precisamente in questo orizzonte: collocarsi nella realtà e saper cogliere in essa quanto è necessario per dare compimento alla propria esistenza. In questo scenario tanto il lavoro quanto la tecnologia sono autenticamente umani se favoriscono tale percorso. La tecnologia in particolare deve essere disegnata in modo tale da favorire, tra gli altri, tre elementi fondamentali: l'autentica scoperta della propria identità personale, come identità donata e nello stesso tempo liberamente scelta nel gioco dell'alterità prima famigliare e domestica e poi sociale. 

Oreste Aime è docente di Filosofia teoretica, Filosofia morale e Filosofia della religione presso la Facoltà Teologica di Torino. Si occupa soprattutto dei filoni fenomenologici ed ermeneutici della filosofia del Novecento, con particolare attenzione al loro rapporto con la religione e la fede cristiana. Tra le sue pubblicazioni: Senso e essere. La filosofia riflessiva di Paul Ricœur, Cittadella, Assisi 2007; Il circolo e la dissonanza. Filosofia e religione nel Novecento, e oltre, Effatà, Cantalupa (To) 2010; Il senso e la forza. Saggi sulla filosofia di Paul Ricœur, Effatà, Cantalupa (To) 2015; I camaleonti. Nuovi luoghi del potere, Marietti 1820, Bologna 2018.

Roberta Aluffi insegna diritto musulmano al Dipartimento di Giurisprudenza dell'Università degli Studi di Torino. I suoi principali campi di interesse sono il diritto di famiglia nei paesi arabi, il diritto dell'immigrazione e le sfide che i sistemi giuridici europei affrontano nell'integrazione dell'islam, nella prospettiva dei rapporti tra lo Stato e le confessioni religiose e del diritto internazionale privato. $\mathrm{Si}$ è occupata inoltre di traduttologia giuridica. 
Luca Battaglini è professore ordinario di Scienze e Tecnologie animali presso il Dipartimento di Scienze Agrarie, Forestali e Alimentari (DISAFA) dell'Università degli Studi di Torino. Le sue attività di ricerca, didattiche e di terza missione riguardano la sostenibilità, anche in chiave etica e socioculturale, dei sistemi di allevamento. Nell'ambito di numerose ricerche locali, nazionali e internazionali, si interessa di impatto ambientale, di caratterizzazione delle produzioni, di biodiversità e di benessere animale, con particolare riferimento ai territori montani.

Roberto Cavallo si è laureato in Scienze Agrarie all'Università di Torino. Già assessore all'ambiente, agricoltura e protezione civile al Comune di Alba, è fondatore e amministratore delegato di ERICA società cooperativa. Valutatore esperto della DG Ricerca della Commissione Ambiente ed esperto prevenzione rifiuti ed economia circolare del Ministero dell'Ambiente. Autore di oltre 80 pubblicazioni e libri sui temi ambientali e naturalistici. Protagonista dei film "Meno 100 chili - ricette per la dieta della nostra pattumiera" di Emanuele Caruso e "Immondezza - la bellezza salverà il mondo" di Mimmo Calopresti. Consulente, autore e conduttore di alcune trasmissioni radio televisive nazionali sui temi ambientali e della striscia quotidiana \# envinews.

Ariel Di Porto ha conseguito il titolo rabbinico presso il Collegio Rabbinico Italiano e la laurea in filosofia presso l'università "La Sapienza", occupandosi delle dottrine non scritte nel pensiero di Platone. Ha svolto per vari anni l'attività di Chazan, abbinandola all'insegnamento presso il Liceo Levi ed il Collegio Rabbinico e alla collaborazione con l'Ufficio Rabbinico di Roma. Dal 2014 è Rabbino Capo della Comunità Ebraica di Torino. Dal 2016 è nel Consiglio dell'Assemblea Rabbinica Italiana (ARI). Collabora con diverse riviste di argomento ebraico. È coautore di un libro di introduzione all'ebraismo e di un testo sulle regole ebraiche di lutto. Partecipa ad incontri volti ad illustrare svariati aspetti della cultura ebraica. Ł̀ attivo nel campo del dialogo interreligioso.

Bianca Gardella Tedeschi è professore associato di Diritto privato comparato, Università del Piemonte orientale. 
Maria Pia Genesin è ricercatrice di Diritto amministrativo presso il Dipartimento di Giurisprudenza dell'Università di Torino. Docente di Diritto degli alimenti, di Diritto urbanistico, di Diritto privato e amministrativo dello sport presso l'Università di Torino; ha svolto attività di ricerca in materia di organizzazione amministrativa, diritto alimentare, diritto dell'ambiente, diritto urbanistico pubblicando svariati saggi in materia. Autrice di due monografie: Lattività di alta amministrazione fra indirizzo politico e ordinaria attività amministrativa. Riflessioni critiche su un sistema di governo multilivello, Jovene, Napoli, 2009; La dialettica pubblico-privato nel sistema della sicurezza alimentare. La tutela della salute fra liberalizzazione temperata e precauzione, Giappichelli, Torino, 2020.

Chiara Ghisalberti ha conseguito la laurea triennale in scienze gastronomiche presso l'Università degli Studi di Parma e ha approfondito le sue conoscenze sulle filiere agroalimentari e sulla sostenibilità grazie alla partecipazione al Master MASRA dell'Università degli Studi di Torino.

Anna Gregis ha conseguito la laurea triennale in filosofia presso l'Università degli Studi di Milano e ha approfondito le sue conoscenze sulle filiere agroalimentari e sulla sostenibilità grazie alla partecipazione al Master MASRA dell’Università degli Studi di Torino.

Paolo Heritier è professore ordinario in Filosofia del Diritto, Dipartimento di Giurisprudenza, Economia Politica e Scienze Sociali (DIGSPES, Università del Piemonte Orientale - UPO). Codirettore della rivista "Teoria e Critica della Regolazione Sociale" e delle collane "Antropologia della libertà"(Mimesis) e "TOB. Antropologia ed Estetica” (Giappichelli). Ha pubblicato La dignità disabile. Estetica giuridica del dono e dello scambio, Dehoniane, Bologna, 2014 ed Estetica giuridica, 2 voll., Giappichelli, Torino, 2012, e un centinaio di articoli sui temi del nesso tra filosofia del diritto e teologia, epistemologia, ermeneutica, humanities, nuove tecnologie, disabilità e informatica giuridica.

Davide Maggi è professore ordinario di Economia aziendale presso il Dipartimento di Studi per l'Economia e l'Impresa dell'Università del Piemonte Orientale. Docente di Eco- 
Alberto Pelissero è professore ordinario di Indologia e tibetologia presso il Dipartimento di Studi Umanistici dell'Università degli studi di Torino, dove insegna Lingua e letteratura sanscrita e Filosofie e religioni dell'India e dell'Asia Centrale. Coordinatore del curriculum di Indologia del dottorato in Lettere della Scuola di dottorato del medesimo Ateneo, è membro della redazione scientifica della rivista internazionale "Historia Religionum" e del comitato scientifico della rivista "Humanitas". Pubblicazioni in volume più recenti: Filosofie classiche dell'India, Morcelliana, Brescia 2014; I cakra, Le ruote d'energia nella tradizione indiana, Magnanelli, Torino 2016; Estetica indiana, Morcelliana, Brescia 2019.

Luca Peyron dirige la Pastorale Universitaria Regionale del Piemonte ed ha fondato e coordina il servizio per l'Apostolato Digitale, uno dei primi servizi a livello globale della Chiesa cattolica che si occupa del rapporto tra digitale e 
fede. Docente di Teologia presso l'Università Cattolica e presso l'Istituto Universitario Salesiano di Torino, tiene un laboratorio di spiritualità delle tecnologie emergenti presso l'Università degli Studi di Torino. Il suo campo di studio è in particolare quello del rapporto tra digitale, fede e società. Collabora regolarmente con alcune riviste e da ultimo ha pubblicato Incarnazione Digitale, Elledici, Torino, 2019.

Paolo Pozzi è medico veterinario; laureato a Milano nel 1988, si occupa di malattie infettive degli animali da reddito, di benessere animale, di audit certificativi di benessere animale in allevamento ed in sede di macellazione. È autore di numerosi articoli su riviste specializzate di settore; nel 2016 ha conseguito la Specializzazione Europea in Animal Welfare Science, Ethics and Law. Ha lavorato sia in Italia che, per un lungo periodo, in Israele e con incarichi di ispettore per il benessere animale presso i locali Servizi Veterinari. E appassionato conoscitore degli aspetti della Legislazione Ebraica (halacha) relativi al rapporto con il mondo animale. Dal 2018 collabora, come Docente a Contratto, con il Dipartimento di Scienze Veterinarie di Torino e presso le Scuole di Specializzazione in Sanità Animale e di Ispezione degli Alimenti.

Amedeo Reyneri è professore ordinario di agronomia e coltivazioni erbacee presso il Dipartimento di Scienze Agrarie, Forestali e Alimentari dell'Università di Torino. Oltre al corso di Coltivazione erbacee, insegna Ecologia agraria ed Ecologia del paesaggio presso il Politecnico di Torino e l'Università di Genova. Dal 2015 al 2018 è stato vicepresidente della Società Italiana di Agronomia.

Lattività di ricerca e di divulgazione riguarda i sistemi colturali e le relazioni tra le tecniche di coltivazione e la sostenibilità del processo produttivo con particolare attenzione alle relazioni tra l'ambiente e la qualità dei prodotti. È consulente presso il MIPAAFT per le azioni della Rete qualità del Piano Cerealicolo Nazionale e del Gruppo di esperti sui contaminanti.

Antonio Sacco è docente di teologia morale alla Facoltà Teologica dell'Italia Settentrionale - Torino, si occupa dei fondamenti dell'etica in prospettiva teologica e del pensie- 
Cristina Videtta è professore associato di Diritto Amministrativo presso l'Università di Torino, abilitata in I fascia. Dal 2005 insegna Diritto dei beni culturali presso l'Ateneo di Torino ed in numerosi corsi di Alta formazione in tutta Italia. Al suo attivo ha altresì la titolarità di insegnamenti universitari di primo, secondo e terzo livello in Diritto Amministrativo, Diritto degli Enti Locali, Diritto dell'ambiente, Diritto Sanitario e Diritto Urbanistico. È intervenuta in importanti convegni e ha preso parte in qualità di esperta a numerosi Master. Per quanto riguarda in particolare la materia del diritto dei beni culturali, è autrice di numerosi saggi tra cui una voce enciclopedica (I beni culturali nel diritto amministrativo) e la monografia Cultura e sviluppo sostenibile. Alla ricerca del IV pilastro, Giappichelli, Torino, 2018. 亡̀ membro del comitato scientifico della Rivista Giuridica di Urbanistica, con cui collabora stabilmente dal 2016.

Giuseppe Zeppegno è dottore di ricerca in Morale e Bioetica; docente presso la Facoltà Teologica dell'Italia Settentrionale - Sezione di Torino. Ha curato con E. Larghero Diritto naturale, dignità di vita e diritti umani (2009); Bioetica e persona 

Ricci: Dignità umana (2016); con M. Rossino Il potenziamento umano (2018); con M. Lombardi Ricci e S. Lepore, Scienza e tecnica. Quale potere? (2019); con E. Larghero e M. Lombardi Ricci, Nascere oggi (2020). Ha coordinato l'area teologico morale del Nuovo Dizionario Teologico Interdisciplinare (2020). Ha pubblicato Bioetica, ragione e fede di fronte all'antropologia debole di H. T. Engelhardt Jr. (2007), La vita e i suoi limiti. Questioni bioetiche (2011), Bioetica e postumano (2017), Il dibattito bioetico da Fritz Jahr al postumanesimo (2020).

Ilaria Zuanazzi è professore ordinario presso il Dipartimento di Giurisprudenza dell'Università di Torino. Insegna Diritto Canonico, Diritto comparato delle religioni, Diritto e religione. Dal 2017 è Presidente del Centro interdipartimentale di ricerca in Scienze Religiose "Eirk Peterson" e dal 2019 è Direttore del Master in Scienze Religiose e Mediazione Interculturale dell'Università di Torino. Ha pubblicato numerosi saggi in tema del diritto interno alle religioni e del diritto dei rapporti tra gli Stati e le religioni. 
aA

stampato

nel mese di luglio 2021

per i tipi di

Accademia University Press

in Torino 